\title{
Social Marketing and Public Health
}

Thesis submitted for the degree of doctor of philosophy (Ph.D.)

Jakob Petersen

Department of Geography, University College London (UCL) 


\section{Declaration}

I, Jakob Petersen, confirm that the work presented in this thesis is my own. Where

information has been derived from other sources, I confirm that this has been indicated in the thesis. 


\section{Acknowledgement}

I would like to acknowledge Professor Paul Longley for his support, personal approach to supervising and encouragement throughout. Thanks to Dr. Phil Atkinson and his public health team in Southwark Primary Care Trust for their valuable input and collaboration. I am also grateful to Maurizio, Dave, Pablo and colleagues at CASA for inspiration as well as advice on analyses and methodology. The PhD research was funded by the Economic and Social Research Council, Department for Business, Enterprise and Regulatory Reform and Southwark Primary Care Trust through a Knowledge Transfer Partnership. 


\section{EXECUTIVE SUMMARY}

The public health field exists to safeguard the general public from health risks by controlling risk factors, classically through immunization programmes that prevent or control epidemics, or through actions such as monitoring the quality of drinking water. In our post-industrialised society, risk factors other than the environment, such as diet, exercise, tobacco and alcohol use, have grown in importance. The policy response to the growing demand upon healthcare services arising from chronic diseases caused by changing lifestyle factors has taking different forms, and these include targeting vulnerable groups using health promoting campaigns.

This thesis addresses some of the challenges and opportunities in public health campaigns and healthcare planning that arise from the growing repositories of data that can be made available for targeting at the individual and small area level in a public health setting.

The first part sets the scene by describing the concepts of health, public health and social marketing. The intention is to pave the way for broader discussions - in the progress of the thesis - about healthcare planning, population health, and social processes in the light of targeted public health interventions.

Part two addresses the problems and possible solutions to a number issues in healthcare planning, starting with studies at the individual, then moving to organisations and ending with area classifications. The thesis draws on a number of case studies for targeting in a public health context including frequent accident and emergency users, teenage users of abortion services, women's breast screening uptake, GP registration, and the neighbourhood characteristics of chronic disease patients. 
Finally, part three provides a synopsis of both context (part one), results (part two) and future perspectives on how routinely collected healthcare data can be used to create evidence for the planning of new cost-effective interventions. 


\section{Table of Content}

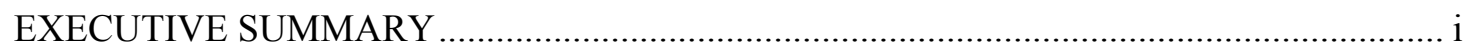

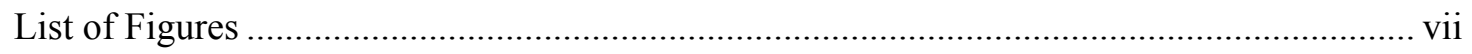

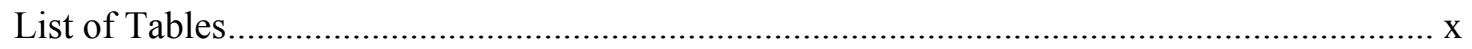

PART ONE: THE EMERGENCE OF PUBLIC HEALTH .............................................. 1

Chapter 1 Concepts of health and a definition of the public health specialism......................... 2

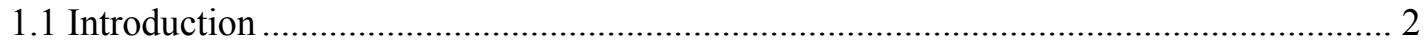

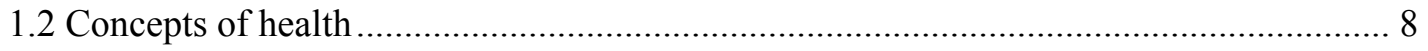

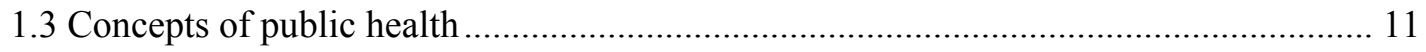

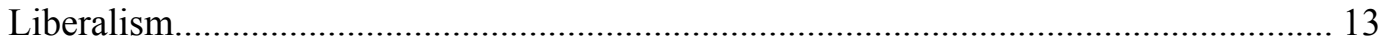

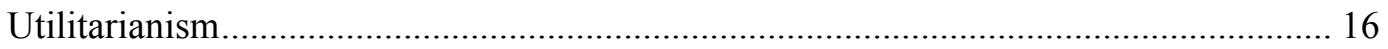

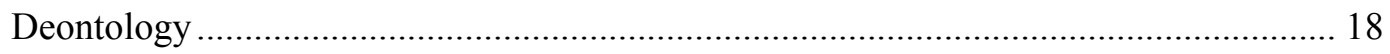

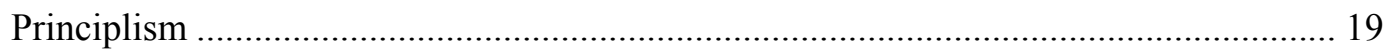

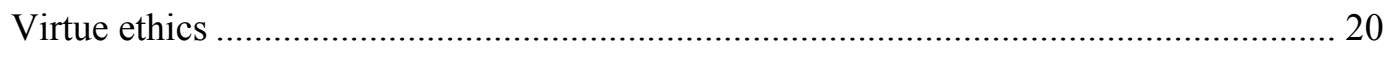

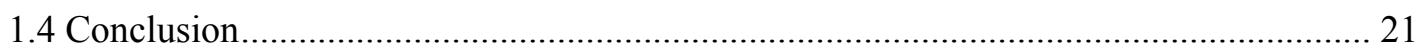

Chapter 2 Political, economic and organisational factors in health policy ............................ 22

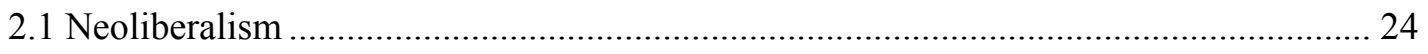

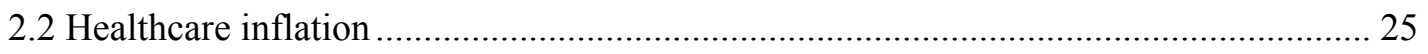

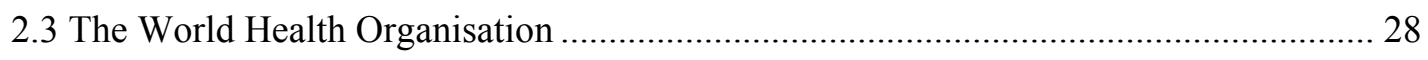

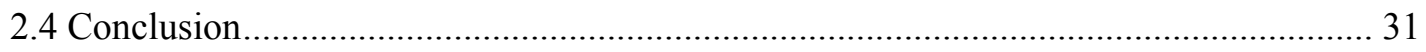

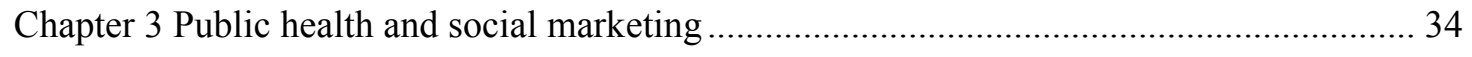

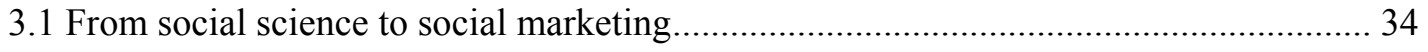

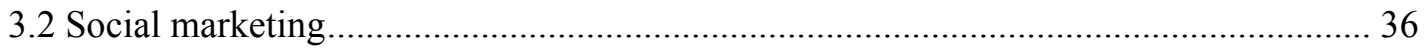

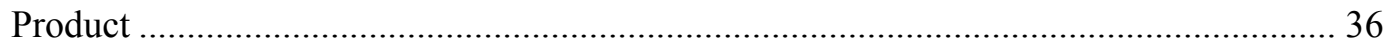




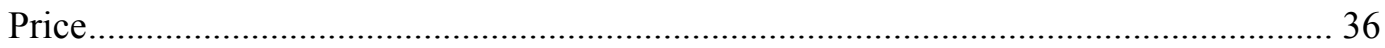

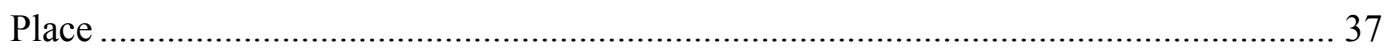

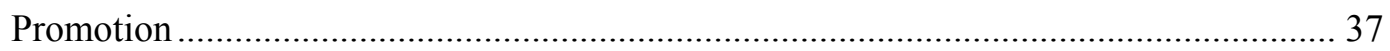

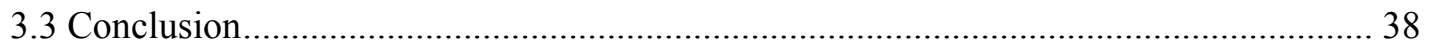

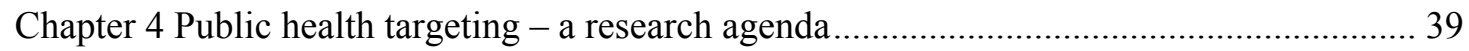

PART TWO: THE GEOGRAPHY OF SOCIAL MARKETING ........................................ 44

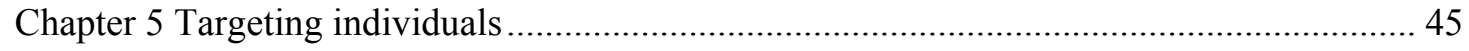

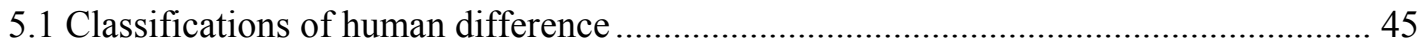

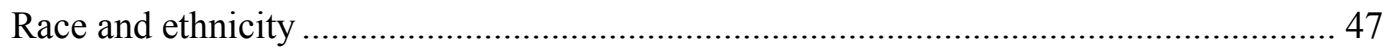

Genome-wide sequencing - a new beginning? ........................................................ 49

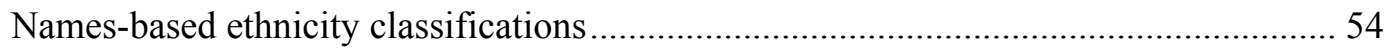

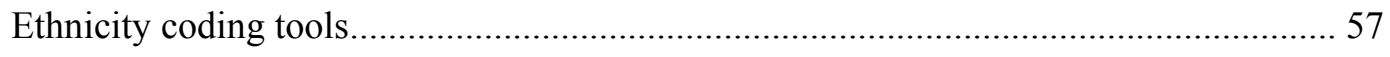

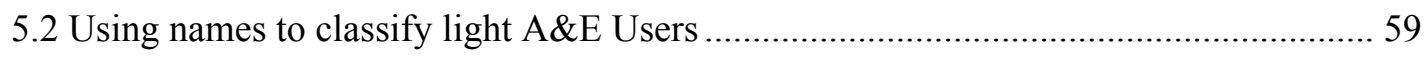

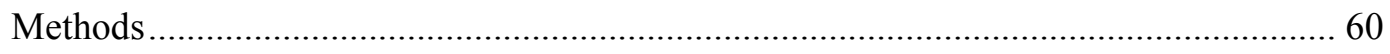

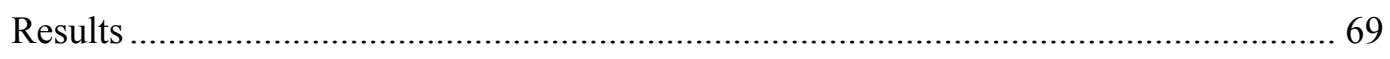

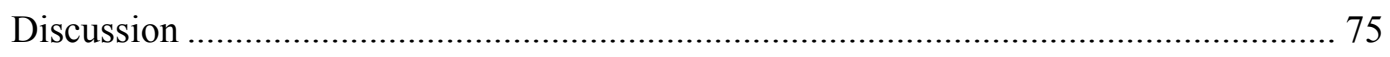

5.3 Using names to understand breast screening uptake ............................................... 78

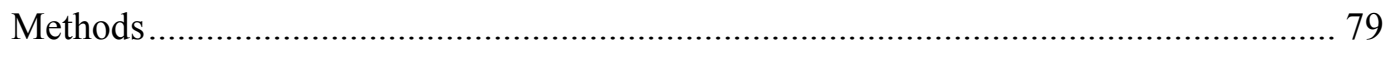

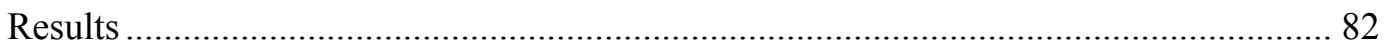

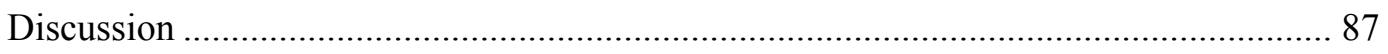

Conclusion - breast screening uptake study .......................................................... 92

5.4 Discussion: ethnicity classifications in public health targeting ................................. 93

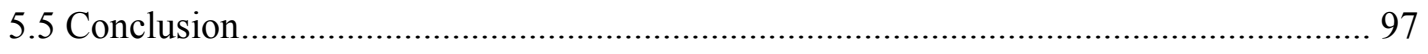

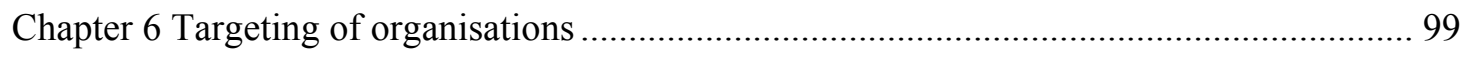

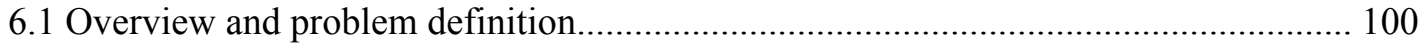

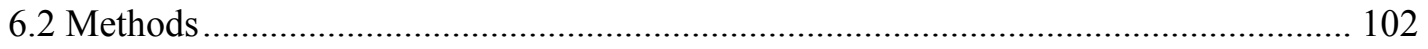




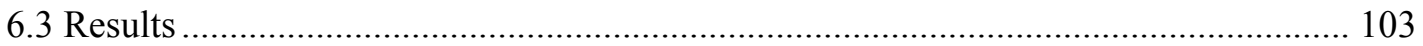

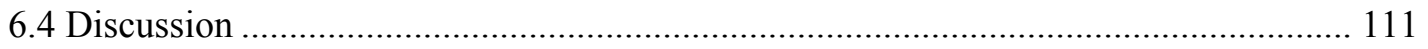

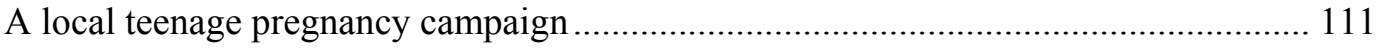

Targeting of organisations in a social marketing context............................................ 115

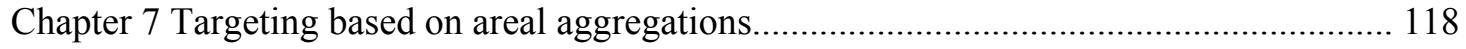

7.1 Health, lifestyles and neighbourhood - a triple construct? ...................................... 119

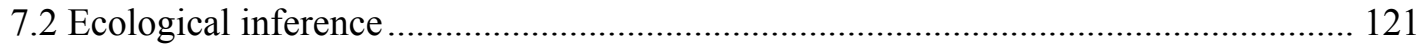

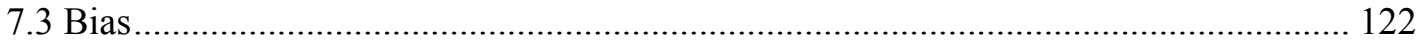

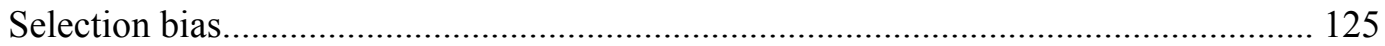

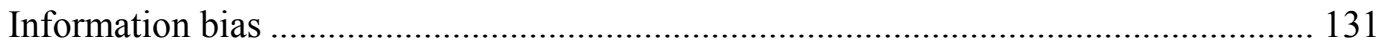

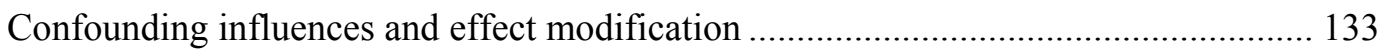

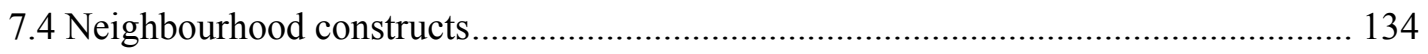

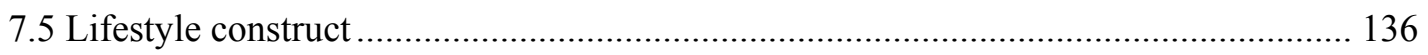

Lifestyle indicators and the lifestyles-neighbourhood 'shunt' ..................................... 139

From lifestyles segmentation systems to geodemographics..................................... 141

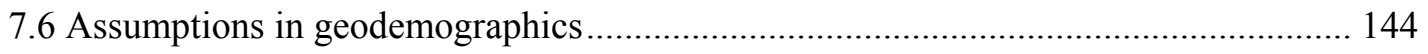

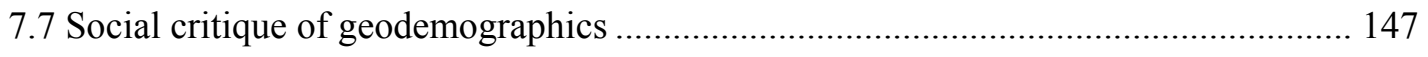

Chapter 8 Neighbourhood classification based on Census variables .................................. 149

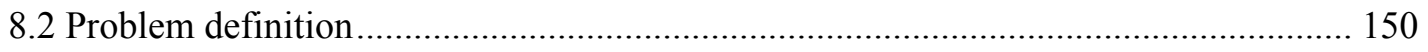

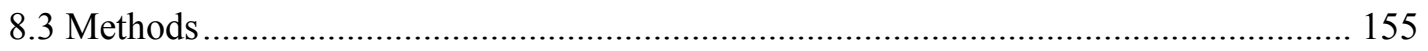

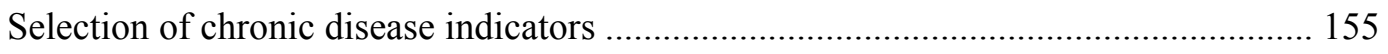

Modification of the Census Output Area Classification for Greater London.................. 156

Geodemographics for neighbourhood targeting - a comparison.................................. 159

Age-standardising geodemographic profiles of health data ....................................... 161

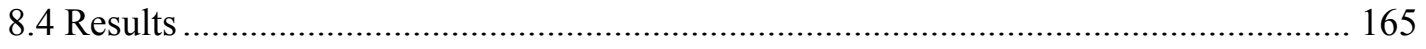

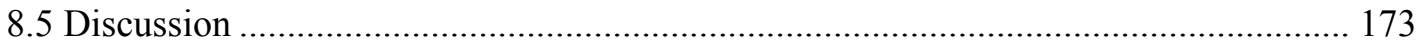




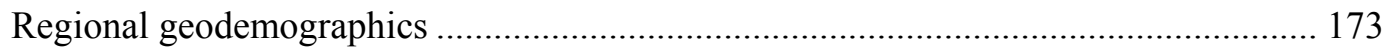

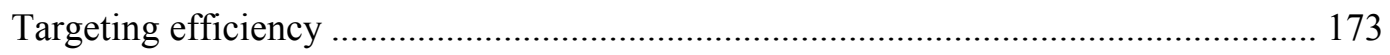

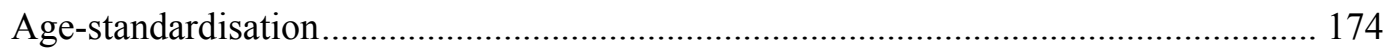

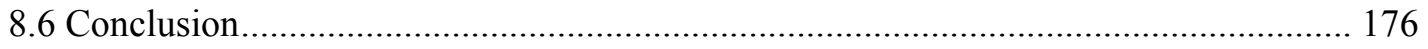

Chapter 9 Neighbourhood classification based on data of hospital demand ......................... 179

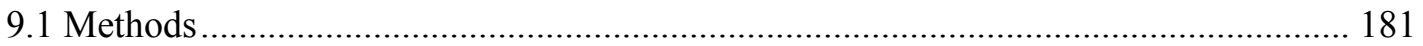

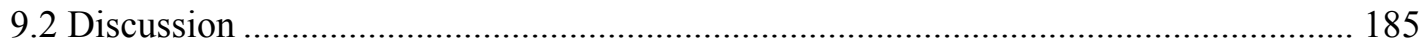

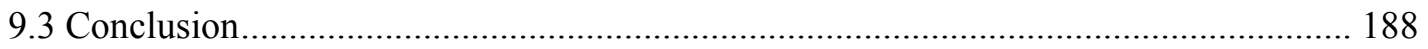

Chapter 10 Information privacy and secondary data analysis ........................................... 190

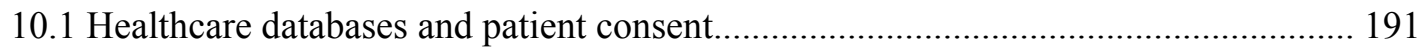

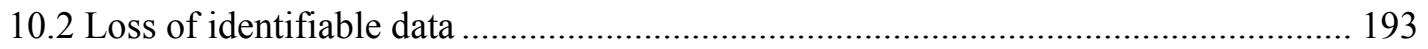

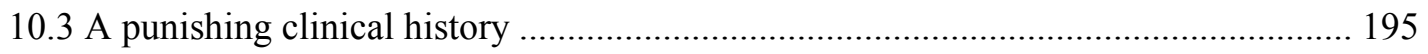

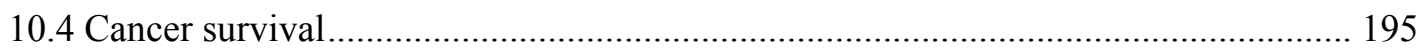

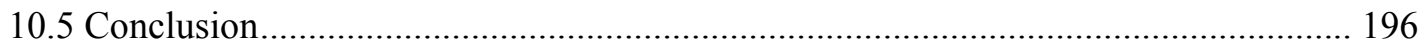

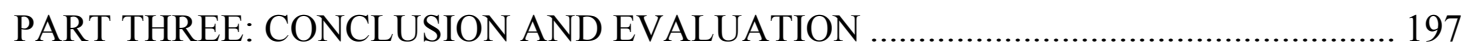

Chapter 11 Contribution to social marketing agenda ......................................................... 198

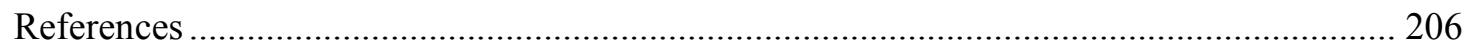

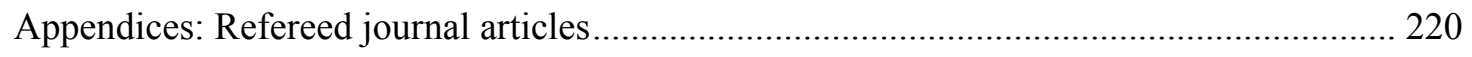

A1: Teenage pregnancy - new tools to support local health campaigns. Petersen, J., Atkinson,

P., Petrie, S., Gibin, M., Ashby, D., Longley, P. accepted at Health and Place

A2: Preventable A\&E usage - The potentials of using a name-based ethnicity classification, Onomap, in identifying user groups at King College's Hospital, London UK. Petersen, J., Gibin, M., Mateos, P., Atkinson, P., Longley, P. submitted to Social Science and Medicine 


\section{List of Figures}

Figure 1 Tobacco marketing (Gardner \& Brandt, 2006) …..................................................... 3

Figure 2 Conceptualisation and formalisation processes in information science ....................... 46

Figure 3 Selection of identifying variables for repeated user based on match rates (\%) of patient attributes as a single variable (cells embounded in black) or in combination of two (upper half of diagram) or three variables (lower half of diagram). Sample source: Southwark patient register, 1 April 2006. Abbreviations: First name (FN), Surname (SN), Date of Birth (DOB), Age in 3-yr intervals (AGE3), names-based ethnicity, Onomap CEL Group category (ONO_GR), residential unit postcode of user (PC). The shading from dark to light in categories of increasing match rates: $<50 \%$ (dark grey), 50-74\%, 75-94\%, 95+\%

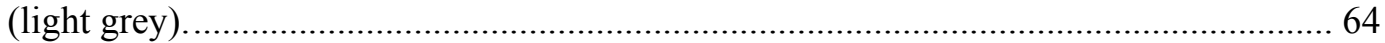

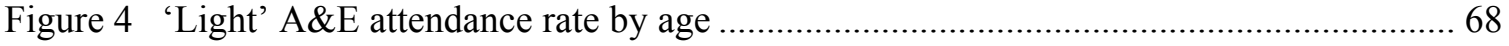

Figure 5 Mortality rate by age, London population 2000. Source: ONS (2005) ....................... 68

Figure 6 Percentage of registered patients with birthplace information in the patient register according to age and time since their registration. Southwark Primary Care Trust patient register, 1 April 2006. The bar in the top left hand corner is the percentage of 0-4 year olds that were registered within the previous 5 years (0-4 years from date of extract) and for which birthplace information were recorded. The bottom right bar is ditto for $65+$ year olds that registered for more than 35 years from date of extract, etc.

Figure 7 Breast screening uptake by names-based ethnicity and birthplace. The analysis of name origin is based on all women and the analysis of birthplace is based on the approx. 50\% women for whom birthplace information is available. Numbers in brackets are the group frequencies. Southwark PCT, 2003-2006.

Figure 8 Breast screening uptake for name origin and birthplace combined. The combined analysis of name origin and birthplace is based on the approx. $50 \%$ of women for whom birthplace information is available. Southwark PCT, 2003-2006.

Figure 9 Breast screening uptake by names-based ethnicity and birthplace. The Mosaic plot shows the relative size of the different groups cross-tabulated for names-based ethnicity and birthplace. Women for whom birthplace information was not available (c. 50\%) were omitted from this analysis. The colour of the tiles in the Mosaic plot shows the uptake rate of each group in tertiles: High 56-73\% uptake in BLUE, Middle 50-55\% uptake in WHITE, Low $32-49 \%$ uptake in RED or insufficient data in GREY $(n<20)$. NB The order of categories is constant, so that e.g. women with an English name is the fourth tile from the top in every birthplace column.

Figure 10 Percent volume contours of teenage conceptions versus under-18 year population. Bandwidth for kernel density estimation were $1000 \mathrm{~m}$. The contours show the top $50 \%$ percent volume contour....

Figure 11 Teenage pregnancy hotspot map showing ONS-released rates (2001-03) and GAM hotspots for the local disaggregated data set. GAM search radius: $250 \mathrm{~m}$, cell size: $50 \mathrm{~m}$.

Figure 12 Recruitment flows and service point estimation (see text for explanation). …......... 107

Figure 13 Gain diagram to reach target group by GP practices or secondary schools............. 108 
Figure 14 Dimensions of healthcare accessibility (adapted from Khan, 1992)....................... 116

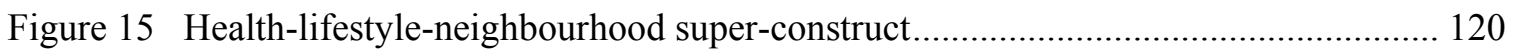

Figure 16 Ecological inference problem in a 2x2 contingency table .................................... 122

Figure 17 Population turnover and healthcare needs. Circles symbolises a neighbourhood at two points in time. Arrows symbolises migration equilibrium. .......................................... 127

Figure 18 Social ties and neighbourhood resilience in five villages in Nang Rong, Thailand. NB order of villages differ in the two panels; ABCDE versus CBAED (From: Entwistle et al., 2007).

Figure 19 Causation in studies of health outcomes. Arrows indicate 'causes' $(\rightarrow)$ and 'associated

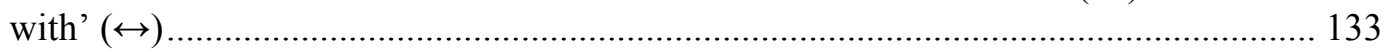

Figure 20 Identity, ethnicity, lifestyle and behaviour modifications...................................... 136

Figure 21 USATODAY.COM content-based readership classification (USA Today, 2008) ... 141

Figure 22 Household segmentation system, Niches Lifestyle system. named from A-Z (Bickert

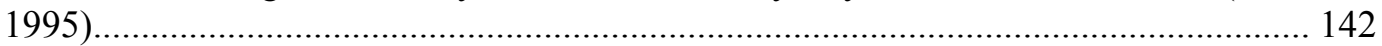

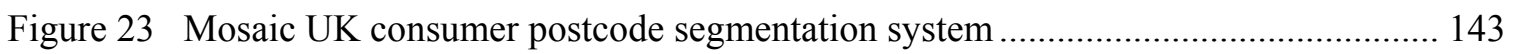

Figure 24 Contacts versus Costs in NHS across service sectors (data from Talbot-Smith \&

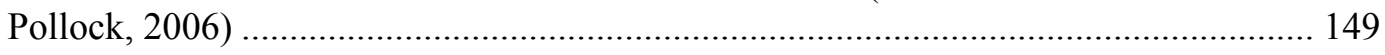

Figure 25 Output Area Classification mapped for Greater London (OAC Supergroups) ......... 151

Figure 26 Stopping rule applied in the creation of London Output Area Classification (LOAC)

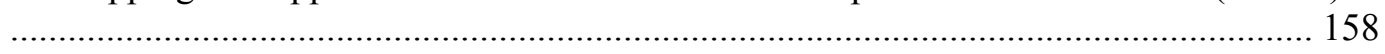

Figure 27 London Output Area Classification (LOAC Groups) ............................................ 158

Figure 28 Median Census attributes for two LOAC 1st tier clusters. Each attribute has been standardised to unity. For the full variable labels see Table 17................................... 162

Figure 29 Age structure in LOAC Groups. ONS population estimates 2001-2004. Top: Male population. Bottom: Female population. NB age bands differ between sexes................ 163

Figure 30 Age structure in hospital admissions. HES Greater London 2001-2004. Top: Male admissions. Bottom: Female admissions. NB age bands differ between sexes............... 164

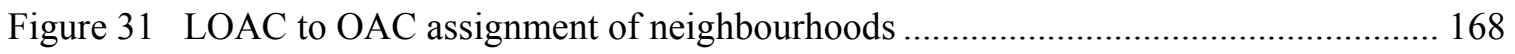

Figure 32 Top: Gini coefficients for chronic diseases across seven different geodemographic systems in horizontal panels. Middle: Sensitivity relative to geographic targeting as gold standard. Bottom: Number-to-target of same.

Figure 33 Geodemographic profile (LOAC) for Angina pectoris hospital admissions. Top: Crude admission ratios. Bottom: Age-standardised ratios. NB Shift in the Suburban category with age standardisation. 170

Figure 34 Effect of age standardisation of hospital admission ratios for key long-term diseases. Male patients in Greater London 2001-2004. Shading: Effects 20 percent below (orange) or above the crude ratios (blue).

Figure 35 Effect of age standardisation of hospital admission ratios for key long-term diseases. Fe60male patients in Greater London 2001-2004. Shading: Effects 20 percent below (orange) or above the crude ratios (blue). 
Figure 36 The geodemographic 'pathway' to health outcomes (see text for annotations)

Figure 37 Geographical distribution of hospital demand of a six long-term conditions. Total admissions per population (middle layer super output area level) classified according to standard deviation, Greater London 2001-2004 (inclusive).

Figure 38 Distribution of clustering variables in London Hospital Admission Classification. Yaxes show bin class fraction. $\mathrm{X}$-axes crude per million person-years-at-risk (prior to log transformation)

Figure 39 Information loss curve for London Hospital Admission Classification. Seven clusters were used $(\mathrm{k}=7)$.

Figure 40 London Hospital Admission Classification. Median of standardised attribute values for clustering variables For explanation to labels see Table 20. 184

Figure 41 Map of London Hospital Admission Classification 189 


\section{List of Tables}

Table 1 Index of Multiple Deprivation 2004 profile of patients registered with a GP in Southwark PCT, April 2006. Mean IMD score is population-weighted

Table 2 Tenure: percentage of households, April 2001 (ONS 2006)

Table 3 Percentage of resident population aged 16 to 74 in each group, April 2001 (ONS 2006)

Table 4 Ethnicity: percentage of resident population in each group, April 2001 (ONS 2006) ... 61

Table 5 'Light' frequent usage of A\&E. Multivariable logistic regression analysis stratified by age

Table 6 GP registration. Multivariable logistic regression analysis of users not registered with a GP. Stratified by age

Table 7 Users not registered with a GP: Name origins (Onomap CEL Type) where more than $25 \%$ of A\&E users were not registered with a GP. Types with $<100$ users included in Other category.

Table 8 Inclination to give birthplace information across names-based ethnicity groups. 82

Table 9 Non-response to Breast Screening. Multivariable logistic regression analysis 85

Table 10 Barriers to breast cancer screening among women from ethnic minorities. Adapted from Remennick (2006)

Table 11 Teenage conceptions estimated for state secondary schools. Schools anonymised: SS\#. Top $50 \%$ of conceptions could be reached by targeting minimum 7 secondary schools (Accumulated percentage: 53\%)

Table 12 Teenage conceptions estimated for GP practices. GP practices anonymised: GP\#\#. Top $50 \%$ of conceptions could be reached by targeting minimum 18 GP practices

(Accumulated percentage: $50 \%$ ) 109

Table 13 Bias types in disease causation. Modified from Delgado-Rodriquez and Llorca (2004) with emphasis on the observational study design. Amendments indicated with *

Table 14 Output Area Classification profile of patients registered in Southwark PCT, April 2006.

Table 15 Mosaic UK profile of patients registered in Southwark PCT, April 2006

Table 16 Number of chronic disease admissions to hospital for residents of Greater London, Hospital Episode Statistics 2001-2004 ........................................................................ 156

Table 17 Census clustering variables .....

Table 18 Geodemographic systems and Neighbourhood differention. NB only segments represented in Greater London are included; this e.g. excludes the Mosaic UK segment 'Rural isolation'. 159

Table 19 London Output Area Classification profile of patients registered with Southwark PCT, April 2006. 167 
Table 20 Median crude hospital admission rates across London Hospital Admission Classification (per 1 million person-years-at-risk). Shading indicates above median rates 


\section{PART ONE: THE EMERGENCE OF PUBLIC HEALTH}




\section{Chapter 1 Concepts of health and a definition of the public health}

\section{specialism}

\subsection{Introduction}

Health policy is central to welfare provision by government. The public health specialism has equipped with the knowledge of social causation and the innovation of the wellbeing concept expanded into any area of the public sector domain of importance for the production of 'good health' and the dissemination of health values (Aday \& Andersen, 1974; Childress et al., 2002; Holland, 2007; Nordenfelt, 2007; Rawls \& Kelly, 2001; Seedhouse, 2004; Sen, 1993). Public health in other words covers not only the discipline of medicine, but overlaps with the remit of the public sector in other domains such as education, youth crime prevention, employment, housing and community relations.

Today, more and younger people are diagnosed with serious and long-term diseases such as diabetes and cardio-vascular diseases following a general transition towards more sedentary lifestyles and richer diets (Bleich et al., 2008; Department of Health, 2004b; Wagner, 1998). The commercial sector has been very successful in appealing to the individual agency of consumers through refined and manipulative advertising. The idea of social marketing is to motivate consumers or citizens to choose 'healthier' options using similar means to those employed by commercial companies (Kotler et al., 2002; Kotler \& Zaltman, 1971); these means persuaded more than $60 \%$ of adult men and $40 \%$ of women to take up smoking in the $1950 \mathrm{~s}$ in both the UK and the USA (Gardner \& Brandt, 2006; Reid et al., 1992) (Figure 1). 
This thesis concerns development and evaluation of new tools for targeting and social marketing in a local setting based on routinely collected data. Public health has a lot to gain from well-placed and professional information campaigns in national media, but local health authorities have a special obligation to reach the most vulnerable groups with specialised support within their jurisdictions, i.e. to reach the 'hard-to-reach'. The intention with the work presented here is to demonstrate the effectiveness and merits of new tools for targeting individuals, organisations and neighbourhoods in support of public health campaigns that address these issues.

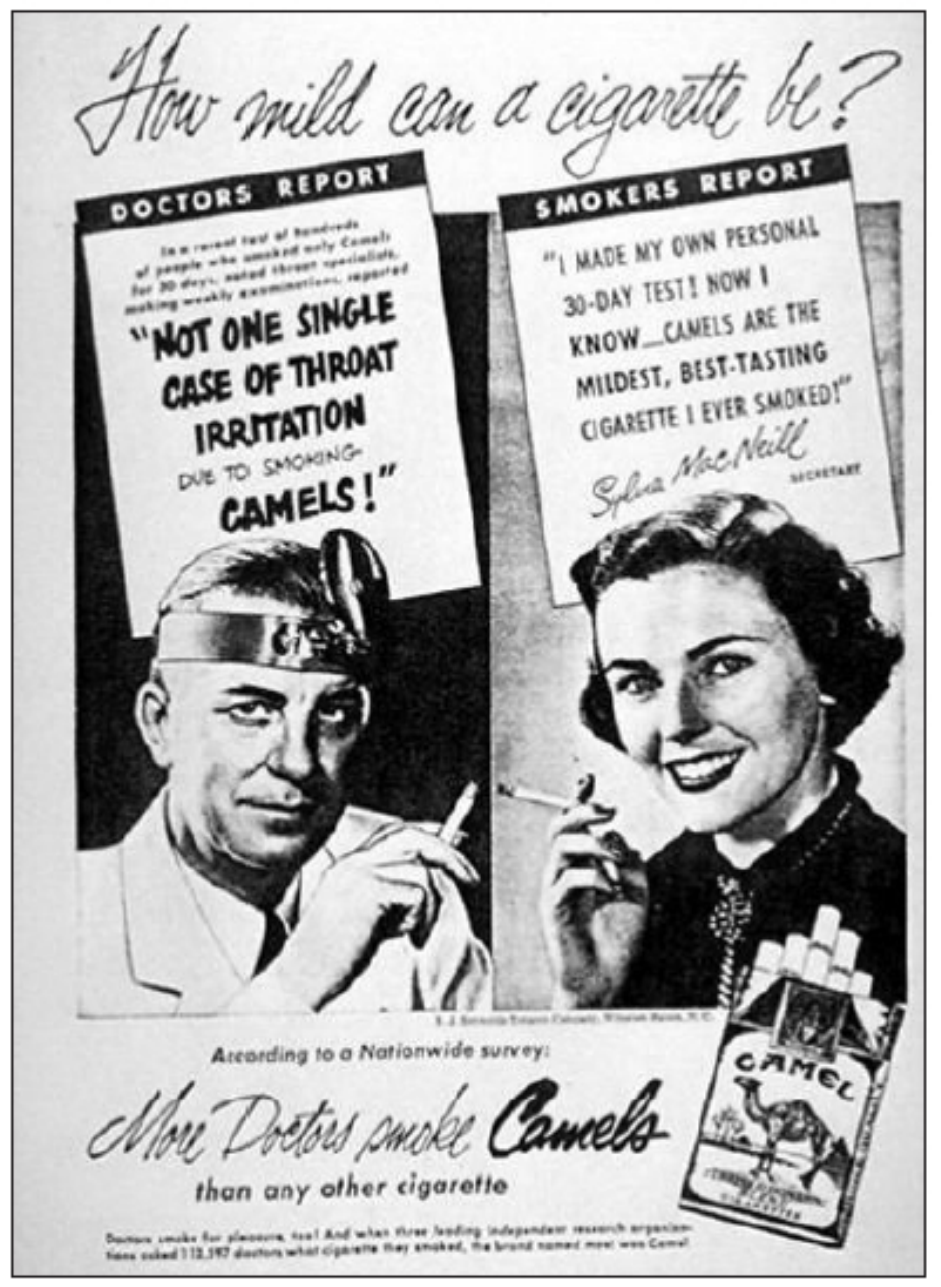

Figure 1 Tobacco marketing (Gardner \& Brandt, 2006) 
There is a growing amount of geographically referenced data available to public health targeting, ranging from Census data and routinely collected data on healthcare service use to geodemographic systems promising lifestyle information at small area level. There are good reasons to examine these data in more detail. Data linkage and fine scale geographical information systems (GIS) based on personal data have vast potential in informing public policy and improving efficiency in healthcare delivery at all levels (Higgs \& Gould, 2001; Twigg, 1989). This development however has also been met with criticism that reflects sensitivities and vulnerabilities that cannot go unaddressed. These cover areas from restriction of civil rights, ethical dilemmas in the prioritisation of public resources, fulfilment of public expectations, increasing 'individualisation' of healthcare, and a general confusion of what the role of public health is. The thesis uses a number of empirical case studies addressing current healthcare planning issues, but also relies on critical analyses of these underlying themes to reach a better understanding of the challenges prior to designing new tools for campaign targeting.

Geographical targeting, GIS and marketing have been accused of only serving utilitarian purposes, or even of only serving the interests of the already privileged, and of not being sufficiently partial in the treatment of the interests of individuals. The same disciplines have also been accused of only adding to the problems of local populations by causing social stigmatisation, 'labelling' and social sorting (Pickles, 1994).

Civil rights campaigners have separate concerns over the centralised recording and linkage of personal information with the resulting threat to privacy. A more subtle viewpoint concerns the type of state citizen would like, see e.g. Lechter (1991);

"While it is generally accepted that each of us is, to a certain extent, 'dangerous to our own health', there is far less agreement on what can or should be done about making people less foolish. In particular, there is the question of how far government should go in fashioning lifestyles to minimize the physical and mental harm we inflict upon ourselves and others in society through risky personal choices" (Leichter, 1991) 
The greater individualism in today's society of 'choosers' (to take a liberalist viewpoint) poses a different kind of pressure on health (and other public) services, where 'users' often are increasingly assertive about their needs and wants and less inclined to accept 'up front rationing'. Health is also individualised in other ways, e.g. in the growing range of product offered to individuals from the 'genomics' industry - be it genetic tests or therapy. What likely impact will this development have on public health decisions?

Public health interventions and health promotion activities often seem struck by confusion.

For whom are they intended? How are the means justified? What are the overall policy aims underpinning them? To exemplify this we only have to look at the philosopher David Seedhouse's almost despairing account of what he calls the magpie profession:

“... health promotion is a magpie profession. Over the years its theorists and practitioners have accumulated countless trinkets from other disciplines, and now possess a stockpile of adopted techniques, models and goals. A glance at the health promotion literature will show that health promoters use booklets (derived mainly from work in medicine and education) to educate patients in hospitals, surveys of people's beliefs about health, illness, wellbeing and quality of life (collected from sociology, psychology and epidemiology), miscellaneous morbidity and mortality figures (from epidemiology and statistics), behavioural change techniques (from psychology), legislative change (from law and politics), lectures and group work in schools (from education), 'look after yourself' exercise and nutrition programmes (from physical education), advertising campaigns (from psychology, politics and propaganda), opportunistic fitness testing (from medicine), joint programmes with food manufacturers to offer approved products and educational materials in supermarkets (from marketing), life skills teaching (from education and psychology), health belief models, health action models, theories of reasoned action (all from sociology and psychology) and a great deal more besides." (Seedhouse, 2004 p.27)

The first part of the thesis aims to set the scene with a critical analysis of the concepts of health, public health and social marketing. The intention is to pave the way for broader discussions - in the progress of the thesis - about healthcare planning, population health, and social processes in the light of targeted public health interventions.

Chapter 1 will examine the ethical foundation of targeted public health campaigns. It will also seek to challenge the view that advances in information technology are purely utilitarian. Chapter 2 will address external factors of importance for health policies, i.e. political, economic and 
organisational factors. Chapter 3 will examine how marketing principles are being integrated with theories of group behaviours and health behaviour modification strategies under the heading of social marketing. Chapter 4 will conclude Part One with a series of research questions that will be formulated on the background of conclusions reached in the preceding chapters.

Part Two addresses problems and possible solutions to a number of current public health issues studied at different scales starting with the individual, then moving to organisations and ending with area classifications. Empirical examples will be drawn from case studies carried out in collaboration with the public health department of Southwark Primary Care Trust (NHS, London) including research with accident and emergency users, teenage users of abortion services, women's breast screening uptake, GP registration, and neighbourhood characteristics of chronic disease patients. Chapter 5 concerns the use of ethnic profiling in the targeting of public health campaigns. The chapter begins with a critical analysis of the classifications as a means of formalising complex information, the concepts of ethnicity and race in studies of health outcomes, the general problem concerning lack of meaningful ethnic information in routinely collected healthcare data, and the use and justification of a new name-based ethnicity classification. The empirical examples in Chapter 5 concern the analysis of frequent users of an accident and emergency department for minor incidences and responding versus non-responding women in a breast screening campaign. Chapter 6 addresses the targeting of organisations as places for fostering specific health behaviour modification programmes. The empirical example concerns the targeting of schools and GP practices in a campaign to reduce unwanted teenage pregnancy. Chapters 7-9 concerns targeting based on areal aggregations. Chapter 7 offers critical analysis of ecological inference, the measurement and definition of lifestyle and neighbourhood constructs as well as important biases in the analysis of health outcomes based on observational and secondary data sources. More specifically it assesses the assumptions underlying the construction of geodemographic systems. Finally, the chapter concludes with an analysis of the social critique attached the use of geodemographic targeting. Chapter 8 addresses some of the 
methodological shortcomings identified in chapter 7 and describes the construction of a new regional geodemographic classification for Greater London. This classification is subsequently compared to six other commercial or publicly available geodemographic systems and area classifications and assesses their relative performance for targeting based on areal aggregations of hospital demand data for 21 common long-term diseases. Chapter 9 explores a different approach to healthcare planning with the construction of a new area classification of composite healthcare needs. Chapter 10 offers a critical analysis of pertinent data privacy issues and safeguards in the analysis of secondary healthcare data.

Finally part three will provide a synopsis of both context, empirical analyses and future perspectives on how routinely collected healthcare data can be used to create evidence for the planning of new cost-effective interventions. 


\subsection{Concepts of health}

A central topic in this thesis is how populations are targeted for public health interventions in ways that are efficient and discuss these in their policy context with concern for ethical, organisational and political economic factors. This section begins with a discussion of how health and public health are defined and open up some of the many dilemmas in the allocation of finite resources among competing needs within a welfare state model. The main theories in political and moral philosophy will here be examined more widely as they apply or are applied in the public health domain.

Let us begin with the World Health Organization's (WHO) definition of health as: “... a state of complete physical, mental and social well-being and not merely the absence of disease or infirmity" and their principle aim as: "the attainment by all peoples of the highest possible level of health" (Preamble to the constitution of the World Health Organisation, 1948). This definition of health has been vividly contested by theoreticians and practitioners alike in their search for ways of justifying or rejecting health policies and practices (Boylan, 2004; Holland, 2007; Law \& Widdows, forthcoming). To neither party does it seem entirely clear what is meant by 'complete physical, mental and social wellbeing' nor for example whether health is to be seen as an end-initself or a means to other things. As a starting point it at least gives us the idea that health can be characterised in two opposite ways; negatively by the absence of something (a disease) or positively by having something (wellbeing). The negative definition is at first notion attractive because it gives a sense of objectivity; i.e. we can concentrate on defining diseases medically as 'deviation from the natural functional organization of the species' and when no disease is found, then the agent is healthy (Boorse, 1975). One of the problems with this definition is that it narrowly assumes that the human species has just two objectives: survival and reproduction. Others have attempted positive definitions emphasising 'wellbeing'; so, that a person can be healthy, because they enjoy wellbeing, even though they have been diagnosed with a disease. 
Conversely, someone else may be disease-free, but nonetheless are not enjoying wellbeing (Holland, 2007). More dynamic definitions of health has been called for in a similar fashion; e.g. not to perceive health and disease as opposite states and to recognise that individuals can have different needs and potentials to cope with adverse circumstances, e.g. Nordenfelt: " $A$ is completely healthy if, and only if, $A$ has the ability, given standard circumstances, to reach all his or her vital goals" (Nordenfelt, 2007). Nordenfelt defines 'vital goals' as 'a set of goals which are necessary and jointly sufficient for minimal happiness' (Nordenfelt, 1995). Seedhouse similarly suggests that 'health is the means to (or resources for, or foundation of) achieving goals' (Seedhouse, 2004). Sen introduce the concept of capabilities; "Capability ... is about what a person is able to do determined by the background social context, the endowments of the individual and opportunities and choices afforded to the individual. ... [it is] the alternative combinations of things a person is able to do or be - the various 'functionings' he or she can achieve. [Capability in this way becomes] ... a set of vectors of 'functionings', reflecting the person's freedom to lead one type of life or another" (Law \& Widdows, forthcoming; Sen, 1993).

Health has also been defined in economic terms, e.g. by Grossman. He describes health as a capital good that will depreciate with age, but can be advanced by various investments, e.g. time spent exercising (Grossman, 1972). In this view agents reluctantly choose healthcare services in order to produce 'good health' and hence increase their personal utility. The economic view on health can seem overly reductionist, but we have to acknowledge that Grossman's work has been very influential in health economics as well as providing a theoretical foundation for areas as diverse as poverty alleviation, facilitation of health insurance and provision of free education. Even if governments could be seen to have narrow economic or paternalistic intentions when urging citizens to choose healthy options, produce health, be productive, be happy, etc., some will question whether citizens always are entirely free to make choices or whether health, lifestyle (and illness) to some extent is embodied in social class and culture (Blaxter, 2003). From the same vantage point it is argued that some citizens may not have sufficient personal resources to 
abandon a given set of 'functionings'. Some of the examples for this viewpoint could be that some young people may start smoking to gain acceptance in a peer group or the apparent acceptance of health among people of working class background as something that simply 'drains away’ (Blaxter, 2003). Others again point to factors early in life and work-related stress as additional factors that are outside the control of the individual (Siegrist \& Marmot, 2006).

Although negative definitions of health can seem less subjective, both negative and positive definitions do not escape being value-laden. The implications of this are that we cannot see health as a universal human right and that when it comes to public health intervention, we would have to ask: "what is being protected and promoted by such-and-such an intervention; i.e., what meaning of 'health' is in play? And what - and whose - values are being reflected in such-and-such an intervention; ones that can be defended and justified, or ones that are glibly assumed to be important and universal?" (Holland, 2007 p.110).

The distinction between positive and negative definitions of health can nevertheless help us create a more nuanced picture of the health concept, bur rather than a 'either/or', the concept, developed for the purpose of the further discussions here, is a 'both/and'. First, in medicine the patient is an individual, whereas in public health the population is the 'patient'. A large proportion of healthcare demands is best thought of as attributable to diseases negatively defined. Second, it is also clear that to have health individuals need certain resources and so health becomes positively defined. Third, another outcome of the positive health definition is a shift from disease to states of wellbeing. As a result of promoting wellbeing public health essentially deals with health as a value-laden concept and consequently decisions have to be sensitive to normative values and beliefs. A typical example of this is where target populations are persuaded to modify a behaviour for prospective health gains such as in tobacco discouragement campaigns.

If we take health, positively defined, as a part of a wider social welfare agenda, then health (and having the resources to produce it) becomes a means to pursue personal goals whether it is do with career, family, or possession of primary goods (Rawls \& Kelly, 2001, p. 58). How and to 
what extent do governments intervene to give all citizens a 'fair' chance to pursue such social welfare goals? How do governments balance the interests of the individual versus the interests of the common good? If we look at preventative interventions; what sort of values and health perceptions are in play? Could a public smoking ban be a restriction of individual freedom? Are sexual health education programmes paternalistic attempts to control young people's behaviour? Are the publication of obesity maps and the targeting of lifestyle campaigns a cruel form of victim blaming or maybe only a way of using vulnerable individuals as mere instruments in tokenistic politics?

In the following the most prominent theories in public health ethics will be examined in preparation of the more specific analyses in the second part of the thesis.

\subsection{Concepts of public health}

To examine the concept of public health this section begins with a couple of definitions from literary sources;

\footnotetext{
"Public health is the science and the art of preventing disease, prolonging life and promoting physical health and efficiency through organised community efforts for the sanitation of the environment, the control of community infections, the education of the individual in principles of personal hygiene, the organisation of medical and nursing service for the early diagnosis and preventative treatment of disease, and the development of social machinery which will ensure to every individual in the community a standard of living adequate for the maintenance of health". (Winslow, 1920)
}

Winslow's definition from 1920 adequately describes the control of infectious diseases. This fits with how public health grew as a discipline throughout the $19^{\text {th }}$ and early $20^{\text {th }}$ centuries to protect the expanding populations in major industrialised cities from diseases such as cholera and polio. Interestingly it also includes a reference to public health as 'social machinery', which could be interpreted as saying, that public health is an instrument to obtain equality and social justice. 
"Public health is primarily concerned with the health of the entire population, rather the health of the individuals. Its features include an emphasis on the [1] promotion of health and the [2] prevention of disease and disability; the [3] collection and use of epidemiological data, [4] population surveillance, and [5] other forms of empirical quantitative assessment; a recognition of the [6] multidimensional nature of the determinants of health; and a focus on the complex interactions of many factors - biological, behavioural, social and environmental - in developing effective interventions." (Childress et al., 2002)

Childress et al.'s more recent definition forms an adequate agenda for a more detailed analysis of public health today. The public health field still concerns safeguarding the general public from health risks by controlling risk factors by means of mass immunization programmes in order to control epidemics or, as another example, monitoring the quality of drinking water (cf.. 2 and Winslow's definition above). In post-industrialised society however risk factors such as diet, exercise, tobacco and alcohol use (cf.. 1) other than the environment has grown in importance. The policy response to the growing demands on healthcare services for chronic diseases caused by changes in lifestyle factors has taken different forms including targeting the population at large with health promotion campaigns (cf. 1). Since the 1970s there has been increasing concern for public policies to be based on scientific evidence; an issue early on championed by Archie Cochrane (Cochrane, 1972; Dobrow et al., 2004). The incorporation of and response to epidemiological evidence is for this reason paramount to modern public health (cf. 3). The recognition of upstream social factors in health, e.g. in the WHO's Alma-Ata Declaration of 1976, is acknowledged in (6) and so other data sources also become valuable to health policy such as Census data (cf. 4) and other data sources (cf. 5), which we can assume include observational data sources such data on doctor's consultations or hospital admissions. Others have raised the point that public health is most clearly justified, where there are benefits for the public as a group, and where these benefits can only be obtained through collective rather than individual behaviour (Dawson \& Verweij, 2007 , p.27). One example of this could be herd immunity, i.e. where high immunization rates in the population are critical for the control of certain infectious diseases. 
The emphasis on prevention is fundamental in public health. For public policy it is thus useful to distinguish between generally immutable, predisposing factors (also referred to as control variables) and enabling factors that are more or less amenable to policy changes (also referred to as policy variables) (Coleman 1971 in Aday \& Andersen, 1974). Aday and Andersen (1974) lists demographic variables such age, sex, ethnicity and health values as predisposing factors and family income and physical access to healthcare as enabling factors for healthcare utilisation. They also acknowledge the potential interactions between policy, healthcare delivery system, population-at-risk, healthcare utilisation and user satisfaction (Aday and Andersen, ibid.). The more important point however for public health policy makers is the need to identify and focus on 'mutable' factors, which concerns; 1) social and behavioural factors relating to the population-atrisk (although Aday and Andersen, ibid., consider health attitudes primarily as immutable), 2) resource allocation and organisation of the healthcare delivery system; and 3) the capability to respond to factors of user satisfaction.

Health is, as established in chapter 1, a concept heavily laden with values that may differ for different users, the ideology or ethical consideration behind public health interventions thus becomes paramount: who is targeted and why, what are the costs in social terms, etc. For this reasons the relatively new field of public health ethics will, in what follows, be scrutinised in more detail. Public health ethics is primarily treated as a sub-discipline of moral philosophy, but the following will begin with a paradigm taking from political philosophy, i.e. liberalism. Liberalism is as a single idea very influential in today's society and it fundamentally deals with the rights and interests of the individual as opposed to social interests.

\section{Liberalism}

One of the most pervasive ideas to emerge from the Enlightenment period is the idea of autonomy; "our capacity for rational self-governance, i.e. the view that persons are distinctive, and distinctively valuable, because they are rational creatures capable of bringing themselves 
under control, unlike mere animals which act instinctually" (Holland, 2007 p.38). The liberal ideas emphasise the value of freedom and the notion that an autonomous individual must be free in two ways; First, they must be free of paternalism, i.e. "[when] those in positions of authority refuse to act according to people's wishes, or they restrict people's freedom, or in other ways attempt to influence behaviour, allegedly in the recipients' own best interests". Second, they must be able to go about their private affairs without interference from the state. This has been termed state neutrality, i.e. the view that "it is not legitimate for the state to make or enforce judgements about how individuals should live their lives". In addition to these points a truly liberal citizen would also be suspicious of state perfectionism, i.e. the view that "some conceptions of the good are better than others and it is legitimate for the state to promote these" (Holland, 2007 p.38). Objections of this kind to public health interventions are common, e.g. banning smoking in public places is anti-liberal, because it compromises smokers' free choice. The idea central to liberalism, that people are best thought of as "individual, atomistic, unembedded, self-standing, rational and autonomous choosers", has however been criticised as incoherent. "To imagine a person incapable of constructive attachments ... is not to conceive an ideally free and rational agent, but to imagine a person wholly without character, without moral depth" (Sandel, 1982 in Holland, 2007 p.40). Holland goes on to explain this view further: “... only people with fully formed characters and moral depth can genuinely choose anything, from a general conception of the good to specific options presented in specific circumstances. One requires the requisite character and depth by being initiated into the culture or tradition maintained by one's community" (Holland, 2007).

Another way in which liberalism has been 'softened up' to public health arguments is the construction of positive freedom, i.e. the idea that liberalism ought not only be negative as in 'freedom from' something, but also positively as 'freedom to' something. In a liberal society a young person might be free to enjoy, what in the eyes of public health would be risky behaviours, say ranging from unprotected sex, binge drinking, smoking, driving without a seat belt to more 
general views of being 'free not to care' or simply be 'free to be foolish' (cf. Leichter, 1991). If this kind of freedom, however means that, e.g. a teenage mother misses out on her education, a well-paid job, an attractive social position, etc. later in life, then it can be argued that state interventions are justified in order to give that individual positive or effective freedom to choose or pursue those primary goods in a lifetime perspective.

The liberalist objections to state interventions are however very strong, see e.g. " ... the only purpose for which power can be rightfully exercised over any member of a civilized community, against his will, is to prevent harm to others" (Mill, 1975 p.14-15). This has been termed liberalism's harm principle, i.e. that the state can and should only interfere to avoid third party harm. It is noticeable that one of the more prominent political arguments in the debate leading up to the ban on smoking in public places in England (put into force 1 July 2007) was health and safety concerns for bar workers, i.e. third party harm.

By now it should be clear that liberalism as a political philosophical movement is not easily done away with, when it comes to justifying state (public health) interventions. Another area where liberalism crops up is in relation to the Universal Human Rights Declaration of 1948. The Universal Declaration of Human Rights covers (among others) rights that are civil and political, viz. the rights to property and liberty. The American and French revolutions were justified on the grounds on infringement of these 'natural' rights and civil rights are central to both the American Declaration of Independence of 1776 and the French Declaration of the Rights of Man and of the Citizen of 1789 (Wellman, 2002). The rights-based declarations of 1948 have admittedly been very influential in the conception of welfare states in the post-war period, but at the same time they have been criticised on a number of points (see e.g. Cowan, 2006);

“... in addressing disadvantage, the human rights model focuses too narrowly on the state as primary duty-bearer. It encourages claimants to demand recognition and resources from the state without acknowledging, given alwayslimited state resources, that choices must constantly be made over whose rights and which rights are privileged over others, and that this prioritization requires serious public debate on rights and needs within the larger collectivity. Focusing energies on legal formalization of rights and the state's obligations 
also diverts attention from other domains, like the economy, that may influence people's lives equally profoundly. Rights are notoriously poor instruments for challenging invidious practices and power arrangements in the private sphere, whether in families or the workplaces of multinational corporations."(Cowan, 2006).

Others again have pointed to the problem of formulating universal rights that are, at least in interpretation, not culturally biased, e.g.

"[The Universal Declaration of Human Rights is]... a paradigm open to interpretation, especially with the presupposition that international human rights standards are culturally neutral. For these reasons the human rights lens is bound to find violations of human rights that point to deficiencies elsewhere. (...) From the beginning human rights are something Euro-Americans take to others. [this problem has been labelled] normative blindness - $\mathrm{a}$ blindness that accompanies a modernization outlook, one that regards pre-modern cultures as a form of backwardness that needs to be overcome. Normative blindness is never more obvious than in Western dealings with the rights of women, elsewhere. For example, the actions and accusations of human rights activists waiting to liberate Islamic women was used as a justification for preemptive war during the Gulf conflict, then the invasion of Afghanistan, then Iraq. (...) Iraqi women under Saddam Hussein were the most equal in the professions like medicine, law and engineering than any other Arab country, and there were more women in engineering classes in Baghdad University that at UC Berkeley during the same time period." (Nader, 2006).

In response to the individualistic liberalism, the next section will move on to another

philosophical movement, utilitarianism (Wellman, 2002). Utilitarianism was initiated or at least

grew as a sceptical counter movement to the violent revolution in France

Rather than championing individual rights (liberalism) utilitarian proponents advocated the interests of the larger collectivity as epitomised in quotes by Jeremy Bentham (1748-1832) such as: "It is the greatest good to the greatest number of people that is the measure of right and wrong".

\section{Utilitarianism}

Utilitarianism can be described as a case of consequentialism, i.e. the moral value of an action is to be determined by its consequences (Boylan, 2004; Dawson \& Verweij, 2007; Hooker, 2008). 
'Right' is hence what bring about the best consequences and 'wrong' is what fails to bring about anything but the best consequences. The best consequences in utilitarianism are those that maximises utility (wellbeing or welfare). In a naïve sense utilitarianism is impartial to any individual interests and as such deals solely with the interests of the group (collectivity or society).

Public health can in many ways be described as a utilitarian endeavour. The principal aim is to protect and promote health as a good for the population as a whole. If resources are scarce (a realistic assumption), a policy that improves the utility of one hundred people, e.g. by funding treatment that will prolong their lives with months, may on some accounts outweigh another policy that spends the same amount of resources on e.g. live-saving cancer drugs for just one patient.

There are different approaches to answering this type of dilemma. Some have suggested a goal of maximising expected benefits rather than immediate benefits, e.g. allocating resources to the treatment of a young person at the expense of an elderly person with the same disabling condition, because the young person potentially would be able to live longer and as such have a greater potential to benefit overall. This type of dilemma has also been termed 'tragic choices' and it occurs more in clinical settings than in public health. An example, more relevant to public health, is the utilitarian approach of cost-benefit analyses. A cost-benefit analysis may balance the economic savings from a smoking ban in healthcare with costs (say, regards to smokers' loss of liberty). This type of analysis has, however, been criticised (cynics of the cynics) for not taking into account the long-term healthcare costs (including the 'cost of dying') for those smokers that otherwise would have led shorter (and happier?) lives (Verweij, 2007). This dilemma can in part be avoided by replacing the savings in healthcare costs for - in this case - diseases related to smoking with measures of years of disability-free living; cf. quality-adjusted life years or QALY (Weinstein \& Manning, 1997). 
Another point of critique is the impartiality of utilitarianism. "Utilitarian allocations aim at the maximization of an outcome over a population and they deliberately ignore the relational and relative differences between individuals" (Rhodes, 2004). Prioritarianism has been proposed as a variant of utilitarianism, where those with the greatest capacity to benefit from an intervention (i.e. needs) should be prioritised for this over those with less or no capacity to benefit (Hooker, 2008). This argument builds on the marginal utility model, e.g. the potential benefit of receiving a bicycle is greater if the receiver had no bicycle beforehand, than if the recipient already had ten bicycles. Although the debate on just allocation of resources within a welfare state system continues with arguments for and against the equality versus priority principles, the prioritarian or 'levelling up' principle has come to be widely used.

A naïve Utilitarian view is - as already explained - not without ethical complications. In a pure utilitarian view it would e.g. be legitimate to ban smoking outright as a threat to health and the maximisation of utility, and in the extreme, it would furthermore be 'right' to shoot a handful of the worst offenders publicly as a visible deterrent for others not to take up similar health harming habits (Holland, 2007). It can be argued that all good public health interventions to some degree are utilitarian, i.e. did they not result in any collective benefits above what could otherwise have been achieved by individual action, then they should not have been carried out to start with. It should also be clear that a public health policy solely based on utilitarian principles is at risk of breaching other values than health maximisation and life longevity such as personal freedom and dignity.

\section{Deontology}

Deontology is derived from the Greek word for duty (deon). It states - in opposition to utilitarianism - that the moral value of an action is in fact independent of its consequences (Boylan, 2004; Dawson \& Verweij, 2007; Holland, 2007). Deontology is heavily influenced be the work of the German philosopher Immanuel Kant (1724-1804). Kant worked with the idea that 
individuals had capacities distinguishable by two unique qualities. First, they are autonomous beings, i.e. they have the "distinct ability to think and act rationally; an ability best expressed [for Kant] ... when they behave morally or as a member of the moral community". Second, they have dignity, i.e. they are "uniquely and intrinsically valuable, and have no exchange value" (Holland, 2007 p.21). From this Kant concluded that people should be treated as ends-in-themselves and never as mere means-in-themselves. In other words; a policy that maximises overall utility at the expense of even one individual would potentially be immoral.

\section{Principlism}

Principlism is the umbrella term for the efforts to find some simple principles for everyday-use of those that practice in healthcare (Beauchamp \& Childress, 2001). In the first instance these principles mainly apply to the physician-patient relationship, but with some modification many of them are also used by the public health specialism (Holland, 2007). The first principle concerns autonomy, which would advise the physician to e.g. obtain consent from the patient before an operation. In public health applications this principle has been extended to a question of how to create rather than to respect autonomy, e.g. informing a smoker objectively about the negative health impacts of smoking could empower that person to quit, whereas respecting his autonomy in this case, would be to leave smoking as a personal matter. Second, non-maleficence, which translates to "do no harm". Third, beneficence, a pledge to help others. Fourth, justice; which tries to deal with balancing individual rights and communal benefits. In the proposed form it leaves a lot of questions, e.g. about who deserves what. Many other principles however have been suggested for public health decisions. Fifth, a reiteration of Mill's third party harm principle; i.e. that the state should only interfere in order to avoid third party harm. Sixth, a principle of least restriction; i.e. among alternative interventions the state should opt for the least restrictive/coercive, e.g. education, facilitation, and discussion instead of manipulation, interdiction, regulation and incarceration. Seventh, reciprocity or fair equality of opportunity 
(Rhodes, 2004); i.e. the principle that the state should facilitate individuals/communities in their efforts to discharge their duties. Eighth, transparency; all stakeholders should be invited to participate and decisions should be made free of vested interests. Finally, the precautionary principle, which translates to: 'when we do not fully understand the impact on the environment of our actions, we should take a precautionary approach' (e.g. used in the United Nations' Rio Declaration of 1992 on global climate change).

\section{Virtue ethics}

Some public health decisions are based on a different kind of judgements described as virtue ethics (Hursthouse, 2008; Jennings, 2007). Virtue ethics originate in the work of Aristotle, who advocated the Doctrine of the Mean, i.e. to find a moderate solution for everything. Popular phrases based on this idea includes: "Too little - too late. Too much - too soon", "in the long term, we are all dead". As an example of how utilitarianism, deontology and virtue ethics differ Hursthouse (2008) give the following example: "Suppose it is obvious that someone in need should be helped. A utilitarian will point to the fact that the consequences of doing so will maximise well-being, a deontologist to the fact that, in doing so the agent will be acting in accordance with a moral rule such as "Do unto others as you would be done by" and a virtue ethicist to the fact that helping the person would be charitable or benevolent" (Hursthouse, 2008). We may conclude that the moral value of a virtuous act is how it is judged - not by rules claiming to be universal like in deontology - but by how it is judged by public opinion in a given and contemporary setting. Jennings gives the following definition; " a virtue [is defined] as a human excellence associated with certain social practices and forms of life ... They are manifested in the way a person engages in social conduct and the normative or rule-governed practices a person pursues, and the excellence achieved within the conduct of the practices as well as the success achieved as a result of applying its powers" (Jennings, 2007; p.46). One could say that virtues are 
about acting 'professionally'. Following similar logic many professions will even publish good practice guidelines for their members.

\subsection{Conclusion}

Although there is such thing as negatively defined health, e.g. manifested in demands exerted upon the medical services for treatment for any given disease, there are many contexts within the public health specialism where health is better perceived as a positive concept, whereby individuals must have a minimum of resources to produce good health or wellbeing, and where this wellbeing as a goal becomes a value-laden concept that should be treated sensitively, and in addition to guidance from moral philosophy should also consider contemporary cultural and political circumstances.

Decisions about public health interventions can have ramifications beyond the remit of health (narrowly defined) and this warrants some critical questions. Can an intervention involve actions that are morally wrong by their very nature? Are individuals, citizens or users in any way treated as means-in-themselves rather than ends-in-themselves (deontology). Can interventions flout important principles, e.g. by not following the principle of least restrictive means? (principlism). Is the proposal virtuous? Or are members of the target population burdened for the sake of political expediency? (virtue ethics). Would a different intervention achieve the same objectives for less and so free up resources for other utility-increasing interventions? (utilitarianism) (cf. Holland, 2007). 


\title{
Chapter 2 Political, economic and organisational factors in health
}

\section{policy}

The essential questions, which will be addressed in this section, concern the principal economic and political factors in public health policy. The healthcare systems are products of the welfare states that emerged in the post-war period. Two different phases of the post-war period, i.e. the consensus phase and globalisation, will be examined;

\begin{abstract}
"[In the first phase] countries of the west settled into a consensus built around a strong state directing the economic and social needs of its citizens, a network of national economies tied together through fixed exchange rates and control on movement of capital and goods, and a military alliance dedicated to the restraint of communism. Western European countries, with their state ownership of public utilities and strategic industries, and their occasional socialist sympathies, seemed radically different from their American partners, but in reality the United States federal government put massive indirect support into its key industries, while Europe was happy to lock itself into an Americanled economic system and military alliance. (...) The second [globalisation] phase began in roughly $1980(\ldots)$. In this phase the inherent value of opening up all aspects of society, and all parts of the world, to private enterprise and to open competition and free markets has been taking for granted. (...) the western military alliance against communism has been replaced by the concept of 'coalition of the willing', formed for specific purposes, while the size and capability of the United States' armed services dwarfs all others. Institutional politic revolves around different ways in which free trade and open markets can be brought into being and managed, while informal opposition, or compensation, tends towards promoting the value of non-tangible assets, such as quality of life, community, environment and religion". (Osborne, 2006 pp.454-455).
\end{abstract}

The construction and maintenance of new post war welfare states however also brought with it some fresh thoughts from economists with the formulation of the public goods theory (Samuelson, 1954). Holcombe sums up public goods as: "a good that, once produced, can be consumed by an additional consumer at no additional cost. ... consumers cannot be excluded from consuming the public good once it is produced. Goods with these characteristics will be underproduced in the private sector, or may not be produced at all [cf. market failure], following the conventional wisdom, so economic efficiency requires that the government force people to 
contribute to the production of public goods, and then allow all citizens to consume them." (Holcombe, 1997).

The theory has assisted in clarifying the likely market mechanisms behind different common goods and in this way given economists tools to analyse how these can be supplied most effectively with or without government intervention.

Goods within a society can be classified into those essential for leading a life effectively (also known as primary goods) to those that are more desirable than essential or socially cultured (Boylan, 2004; Rawls \& Kelly, 2001). The exact boundaries for what a welfare society should provide its citizens is to a large degree (barring primary goods) dependent on values and what is perceived as acceptable within that society and so goods very much become politically contestable. A different angle on this debate is to examine the equalitarian principles behind welfare policies. In which way should citizens be equal? Sadurski sums up this debate as equality-of-welfare (the outcome) versus equality-of-resources (the means) (Sadurski, 2007). He describes three parts to this question; individual versus societal interests, persons versus circumstances, and 'luck' versus choice. Equality-of-welfare is utopian and - it has been argued undesirable as it would eliminate individual choice (and motivation). Proponents for equality-ofresources argue that choice and - to a degree - 'luck' should be preserved and that individuals instead should be held responsible for their own choices.

While it is easy - for the sake of explanation - to reduce public services to questions about fairness and equity and only secondary to include public finance, governments in reality continuously experiment with different funding models to meet the rising demand and expectations to public services. The following section will visit aspects of the main political and economical models used in healthcare provision policies. 


\subsection{Neoliberalism}

The political climate following the Second World War has been greatly influenced by ideas

about the market-oriented economy or neoliberalism. Harvey defines neoliberalism as follows;

"Neoliberalism is in the first instance a theory of political economic practices that proposes that human wellbeing can best be advanced by liberating individual entrepreneurial freedoms and skills within an institutional framework characterised by strong private property rights, free markets and free trade. The role of the state is to create and preserve an institutional framework appropriate to such practices. The state has to guarantee, for example, the quality and integrity of money. It must also set up those military, defence, police and legal structures and functions required to secure private property rights and to guarantee, by force if need be, the proper functioning of markets. Furthermore, if markets do not exist (in areas such as land, water, education, healthcare, social security, or environmental pollution) then they must be created, by state action if necessary. But beyond these tasks the state should not venture. State interventions in markets (once created) must be kept to a minimum because, according the theory, the state cannot possibly possess enough information to second-guess market signals (prices) and because powerful interest groups will inevitably distort and bias state interventions (particularly in democracies) for their own benefit" (Harvey, 2005, p.2).

Neoliberalism has been criticised as not being a complete ideology (unlike liberalism, for example), but rather a set of ideas that has strongly influenced post-war politics (Thorsen \& Lie, 2007). Taking 'as-is' neoliberalism would arguably be a very radical ideology with potentially dire consequences on a number of key policy areas unless accompanied by some degree of central regulation. Health is one such area where most citizens would require protection, e.g. against unexpected health risks commanding expensive treatment that would not be affordable for the uninsured. More specifically with regards to UK then the NHS has since its foundation in 1948 provided care that aims to be universal, comprehensive, and free at the point of delivery. This is not to say that there are no restrictions as to what treatments might be available at particular points in space and time, e.g. the public debate about what defines 'core' versus 'non-core' healthcare services is very much alive (Clarke et al., 2008). Many of the NHS reforms since 1980, although proposed and implemented by governments of different colours, can retrospectively be seen as adaptations to neoliberal ideas about creation of a market in healthcare. This market would ideally create competition such that only the most cost-effective 
organisations/subcontractors would survive. This development is evident not least in the New NHS Plan from 2000 (Department of Health, 2000) or the new contracts between the Department of Health and the independent GP practices (Department of Health, 2004a). In relation to this thesis this development emphasises the need for new information technologies to improve the cost-effectiveness of social marketing campaigns as well as the targeting of healthcare service resources whether for short or long term gains.

\subsection{Healthcare inflation}

Most developed countries have experienced a gradual rise in healthcare expenditure since the 1960s (Organisation for Economic Cooperation and Development, 2005). With ageing populations this healthcare inflation is predicted to worsen with fewer of working age to support the funding of healthcare systems that at the same time is expected to see a growing demand for their services (Gray, 2005). In the UK the healthcare system, National Health Service (NHS), is principally funded by the government and as such is central to political debate about not only the extent of taxation, but also questions of its share of the state budget in competition with other public sectors like education, policing, social security, defence, etc. (Hsiao \& Heller, 2007). Within the healthcare system there are again complicated trade-offs between equity and efficiency in improving population health, reducing risks and inequalities, and a need to ration services balanced with maintaining a certain level of user satisfaction (Aday \& Andersen, 1974; Musgrove, 2003). There is for these reasons an increasing pressure to reform healthcare systems and the NHS is undergoing reforms to make its organisation more cost-effective and also to attract private enterprise (Pollock et al., 2007; Talbot-Smith \& Pollock, 2006). The current model - in which NHS is organised in a hierarchy of administrative levels from Strategic Health Authorities, Primary Care Trusts and GP practices - is expected to be slimmed down, so that most healthcare services by 2010 will be commissioned (i.e. purchased) by GP practice managers and independent hospital trusts will be competing for patients. A new agency, Monitor, will be acting 
as the government's 'watchdog' and intervene if patients' interests are compromised in this quasifree market. Prices for treatments will however be fixed by the Department of Health. 'Survivors' in this market are predicted to be those that are able to minimize costs, while maximising patient satisfaction. One immediate concern with this arrangement would be that the artificial price setting may create situations with perverse outcomes, e.g. - depending on the current year's pricelist - some patient groups may be more 'lucrative' than others. As a consequence, this 'marketisation' of healthcare may, if not carefully designed, end up with perverse outcomes, e.g. failing rural areas due to monopolisation (i.e. market failure caused by a spatial effect). Examples of this type of inverse care law cases have already been highlighted by several other studies, where healthcare providers tend to locate disproportional to needs (Hart, 1971), e.g. where there are greater opportunities for private consultancy (Shaw \& Dorling, 2004) or where vulnerable patients potentially can be let down by cost-cutting in other public sectors, e.g. if subsidised bus services are reduced in rural areas it may effectively cut off many vulnerable elderly from seeing a GP (Lovett et al., 2002).

Technological advances are often considered to be the prime cause of healthcare inflation. This is because, the argument goes, that patients increasingly would demand the latest and most expensive treatments and that physicians would recommend the same in the name of scientific progress. It is noticeable, however, that whilst new technology has been introduced simultaneously in most developed countries, healthcare inflation has been much more pronounced in the USA, where healthcare is funded by private insurance companies, and less so in the statefunded welfare countries (Hsiao \& Heller, 2007). This suggests the healthcare inflation has more to do with open versus closed-budget systems (free market versus state monopoly with budget capping) than the introduction of new technology (Hsiao \& Heller, 2007).

The rising costs in the public sector, especially in education and health, have thus been met with different responses from governments although the question is rarely a clear-cut: pro- 
nationalisation or pro-privatisation of public services, but ever changing models mixing different means of funding. The reforms of the NHS since the early 1980s seems on the whole inclined to a neoliberal model, where healthcare service goods have been produced in a regulated 'marketplace' with an increasing involvement of private finance. Another 'trend' for NHS service strategists seems to be reducing 'unnecessary' demand. A recent contribution to this debate has been crafted by Charles Clarke, member of parliament for the Labour party and former Home Secretary, and Klynveld, Peat, Marwick and Goerdeler finance advisory company (Clarke et al., 2008). In this paper they advocate for not reducing demand, nor encouraging privatisation, but to increase user charges for 'non-core' services in the public sector (including healthcare) in order to gain better public services in the face of the public sector funding crisis. Their ideas would diminish the apparent problems with 'market failure' in pure privatised system such as in the USA, where the value of services seems to erode at the expense of users and company shareholders emerging as the main beneficiaries (Hsiao \& Heller, 2007). Users would under this scheme be able to enjoy 'core' services from the same facilities as for 'non-core' services, e.g. 'core' life-saving surgery and 'non-core' cosmetic surgery, whereas they at present would seek private alternatives for the 'non-core' services instead. The potential revenue from this source is backed up by estimates for private healthcare expenditure in the UK; for which the latest estimate is $£ 39$ billion per year (Clarke et al., 2008). The NHS budget in 2006-2007 was for comparison $£ 83$ billion per year (Department of Health, 2005a), which means that all else equal every third pound spend on healthcare at present goes to private healthcare. The main problem with user charges, whether they are called taxes (e.g. road tax), duties (e.g. on tobacco), top-up fees (for students in tertiary education), licence fees (e.g. TV or driving licences), congestion charges or access fees (e.g. in public museums) is that they 'hit' the poorest sections of the population disproportionably to the more affluent sections of the populations with implications for equity. Clarke et al. concedes that 'dealing equitably with the implications of a system of user charging is not straightforward' and 'In general a system of making adjustments to charges, through means 
testing, might be most justified where there are overall benefits to society, such as reasonably equal access to healthcare for all' and finally that 'Whilst offsetting arrangements can be made for particular disadvantaged groups [e.g. means testing], these are usually administratively complex and do not always target the right people' (Clarke et al., 2008).

\subsection{The World Health Organisation}

The following section begins by addressing international relations in health policy centred on the creation and short history of the WHO since 1948. It then proceeds to examine how international health policy is influenced by political and economic factors in addition to ideological factors. The first international conference on health was held in Paris, 1851. The pretext for the conference was the cholera epidemics in Europe during the 1830-1840s (McCarthy, 2002). International health or sanitary organisations were later established to cover the American continents (1902) and Europe (1907). These early initiatives grew out of a common desire to control the spread of infectious diseases between the great powers and their colonial counterparts. The first worldwide health organisation was established in 1919 in conjunction with the foundation of The League of Nations. The present WHO was established alongside the United Nations (UN) in 1948 - its sister organisations for international law, international security, economic development, social progress and human rights issues. The preamble to WHO in many ways reflects the UN's universal declaration of human rights from the same year, e.g. in reference: "the attainment by all peoples of the highest possible level of health" (Preamble to the constitution of the World Health Organisation, 1948).

Health objectives remained in the first decades devoted to medical prevention, but possibly as a reflection of growing health inequalities in economically developed countries upstream factors such as housing, education, crime, environment, etc. became more explicit parts in the health policies of the 1970 s. 
During the consensus period social welfare programmes were established in developed countries and these also became the model for international development. Healthcare policies were however first of all concerned with clinical aspects. The general lack of improvements in health inequality in developing countries and at a relative scale in developed countries too (Department of Health and Social Security, 1980; Townsend, 1979) brought about a change of tack. This is evident from the Lalonde report (1974) and the WHO Alma-Ata Declaration of 1976, where public health was proposed as a new direction for healthcare policy in addressing upstream social factors and to stimulate community participation and intersectional collaboration. These ideas and since been elaborated, e.g. in the WHO programme, Health21 (World Health Organisation, 1998) to also include action for sustainable development;

1) Equity. Promoting equal opportunities for health and healthcare including action to combat poverty and social exclusion. Governments are also urged improve the health of minority ethnic groups.

2) Community participation. Promoting the capacity of local people to participate in action for health and decisions affecting their communities.

3) Intersectional collaboration. Taking action for health by working cooperatively with a range of governmental and non-governmental (NGO) commercial organisations.

4) Sustainable development. Environmental strategies, e.g. energy efficient transport and housing

The greater ties of world-wide economies in the globalisation era raise new implications for health and social welfare that lies beyond the boundaries of nation states. National governments are facing new challenges and there is a growing focus upon so-called global governance; "the complex of formal and informal institutions, mechanisms, relationships, and processes between and among states, markets, citizens and organizations, both inter- and non-governmental, through which collective interests on the global plane are articulated, rights and obligations are 
established, and differences are mediated" (Thakur \& Weiss, 2005). This definition allows us to expand the debate from the defence of welfare rights to a formal recognition of the role of multiagency, whether individual, governmental or non-governmental, economic or ideological, and the importance of mediation of conflicts involving all of these actors.

There are different theories on how global governance can assist to regulate political processes. Drezner describe these as either driven by economic circumstances (material pressures) or ideology (ideational pressures) in one dimension and in a another dimension to whether actors are relatively free to act or are more constrained (Drezner, 2007). Out of the many theories made for outcomes of the globalisation, two contrasting theories, race-to-the-bottom and Global Civic Society, will be covered here. The former theory predicts that welfare rights over time will deteriorate in a lose-lose situation, while the latter predicts that social welfare rights increasingly will be defended and won in transnational fora of power.

The protection of patents for antiretroviral drugs (needed by HIV/AIDS patients) is an interesting case for public health policy in the era of globalisation, for several reasons. Without patents the pharmaceutical industry would not have any economic incentives to develop new drugs. Sub-Saharan countries however are particularly hard hit with nearly $60 \%$ of adults infected (UNAIDS, 2006). The same region has $64 \%$ of the world's HIV patients of which few would be able to afford imported goods let alone patented antiretroviral drugs. This is not, as it may appear at the outset, simply a clash between ideologies; between believers of the state guaranteed social welfare in one corner and proponents of the free markets in the other. Patents themselves create monopolies and are essentially barriers to the free entry to a market.

An alliance, the Access Campaign, was formed in opposition to the pharmaceutical industry and was joined by many low income countries as well as transnational NGOs including wellknown organisations such as Médecin Sans Frontières and Oxfam. The Access Campaign successfully lobbied the great power governments and international governmental organisations to support a World Trade Organization declaration in Doha 2001 to remove the 10 year patents on 
a list of drugs essential to public health and allow the trade of cheaper copy-products (Drezner, 2007). The success for the alliance of low-income governments and NGOs has been interpreted as evidence for the Global Civic Society, i.e. that the social welfare rights campaigns can be taken to the global stage. It moreover showed that fora for international economic relations like the World Trade Organization (WTO), International Monetary Fund (IMF) and World Bank, may become more important for international healthcare policy that the WHO itself (Drezner, 2007). MacLean cite similar cases affecting public health, e.g. coalitions behind campaigns to counter the illegal trade in tobacco or the international ban on landmines, but concludes maybe wishfully that: “... although WHO is impeded, like other UN organizations, by limited resources as well as by internal struggles that are factors of both international politics and institutional competition, it does play a major role in the construction of international norms on health. It may also begin to play a larger role setting norms in the broader area of human security not only through its involvement in ... various coalitions ... but also in its recent attempts to bring attention to the relationship between health and trade. ... The shift from a predominantly clinical approach to health to one that addresses political economy factors is a major step forward in improving the social determinants of health" (MacLean, 2007, p.145).

\subsection{Conclusion}

Although the initial pretext for international relations in health politics was the control of communicative diseases, the WHO has become the main organisational driver for health policy. With the WHO Alma Ata Declaration of 1976 public health aspects of these policies have broadened to include 'upstream' social factors in health and wellbeing and a new emphasis on community participation, intersectional collaboration and (later) also sustainable development. The Globalised economy poses new challenges for health policy, but because of their economic rather than ideational nature, fora for international economic relations like the WTO, IMF and the 
World Bank, are becoming more important for international healthcare policy than the WHO itself

A central issue in the development of welfare governing since 1945 has been the production and distribution of public goods including those improving citizens' health. There are several issues in this. First, the notion of 'fair' distribution under scarce resources is in reality hard to define. Are policies intended to 'level up' in fact levelling the citizenry 'down' and if the overall aim is equality of welfare, would that entail the demise of personal choice as a motivational factor? Alternatively, equality-of-resources has been advocated as a more viable aim. Second, whilst public goods are intrinsically linked to economic factors, not least for their funding, public goods theory points out that they would be critically under-produced in a pure free-market economic system, and so become dependent on government regulation and agency. While fewer question the value of public healthcare systems, there is a continuing debate about whether public goods should remain 'public' in their production or whether governments should rather encourage free market mechanisms in which they would act as purchasers of various services on the behalf of their citizens. Third, healthcare systems are facing a funding crisis (also known as the healthcare inflation) caused by ageing populations, a rise in lifestyle-associated long-term diseases, and relatively fewer of working age to fund the rising costs through tax revenues. This implies complicated trade-offs between equity and efficiency for the bettering of population health, reduction of risks and inequalities, and a need to ration services balanced with regards to user satisfaction. The use of budget capping in national healthcare systems (in response to the healthcare inflation) seem to be stimulate greater cost-efficiency than what seems to be the case with the 'open-budget' healthcare market system of the USA.

User charges are already used in healthcare, e.g. in prescription charges, and considering that up to a third of UK healthcare expenditure could at present be going to the private sector there should be scope for widening NHS services to not only meet essential 'core' needs, but also increase efficiency by tapping into this 'non-core' market. The present ideas for user charges 
however are still problematic for equity questions - not least because of the administrative costs that inevitably would follow with e.g. means testing schemes.

The last points again emphasises the need for healthcare planning tools that would deliver better efficiency and equity for many of the current healthcare issues; a topic that will be pursued further in the part two of this thesis. 


\section{Chapter 3 Public health and social marketing}

As discussed in the previous chapter health policy has, since the WHO Alma Ata Declaration of 1976, broadened to include a wide range of 'upstream' factors; to mention the most common; housing, education, employment and environmental quality - in fact anything to do with the public sector sphere. Moreover as discussed in the chapter on health concepts it can be argued that we as individuals are 'dangerous to our own health', and so any self-respecting public health department would not hesitate to venture into to the private sphere of people's lives and educate them about the dangers of their desires and preferences - their 'lifestyle choices' (cf. Leichter, 1991). The implications of this are twofold. First, public health decision-makers have to analyse and understand the social processes and environments causing diseases and other utility-reducing health outcomes in order to design across-the-board policies. Second, in order to influence local populations through intelligent health promoting campaigns they similarly need to be familiar with their 'audiences' with regards to needs and health behaviours. The following section will outline the principles of social marketing and its application in the public health specialism.

\subsection{From social science to social marketing}

Before the discussion into social marketing a slight detour will be taken into social science in order to expose a more profound point. Social science is 'the study of human society and of individual relationships in and to society' (Dictionary.com, 2008). Often the information we have available on populations is measured naturally at group level (e.g. air pollution or housing), or the data are aggregated (e.g. due to data privacy issues). Most public health interventions are targeted at the asymptomatic population at large or population groups with particular needs. For this reason it would be prudent to consider a couple of ideas central to social science before moving on to social marketing. 
One of the more difficult, but central topics in social science, is how cause and effect of phenomena of social interest can be untangled. If we take the claim: "Individuals belonging to the same group behave similarly", then this could be explained in two different ways (Manski, 1995);

1) Endogenous effects; the behaviour of an individual depends on the group. This effect is sometimes also referred to as peer pressure;

2) Correlated effects; individuals respond similarly but independently in response to the same external pressures/environments or they behave similarly because they have similar individual characteristics.

An example;

a) $A$ has a health-damaging alcohol problem, because heavy drinking is the norm where $A$ is (at home, in the neighbourhood, in the workplace, or as a part of $A$ 's social life).

b) $A$ has an alcohol problem, because $A$ has a stressful job.

The remit of public health is in theory wide ranging, but what is the easier option; to change behaviours that are endogenous or correlated? Could new all-powerful and all-encompassing public health implement policies that would make the lives of the citizenry less stressful (correlated effects) and then slowly reap the benefits from fewer alcohol-related hospital admissions? Or should public health target endogenous behaviours by e.g. making it 'cool' to drink a non-alcoholic drink like orange juice instead? The latter seems to be the easier alternative although changing people's desires and preferences is no easy task in itself (see e.g. section on lifestyles, section 7.5, p.136). As discussed earlier health promotion comes close to perfectionist state interference with citizens' freedom to make choices for themselves. And so it should also be mentioned that there are other approaches, e.g. by 'sin-taxing' alcohol and tobacco, which would be a restrictive rather than a distributive policy. 'Sin-taxing' is nonetheless rarely popular with the electorate and can in addition be seen as a broad sweep and proportionally less fair towards low income groups. 


\subsection{Social marketing}

Social marketing was first defined as: "the use of marketing principles and techniques to influence a target audience to voluntarily accept, reject, modify, or abandon a behaviour for the benefit of individuals, groups or society as a whole" (Kotler and Zaltman 1971). Social marketing uses terms familiar with marketing and many campaigns are based on the so-called 4Ps: Product, Price, Place and Promotion. Below each $\mathrm{P}$ will be interpreted as applicable to public health.

\section{Product}

All marketing builds on the principle of exchange, i.e. tangible goods or a service in exchange for money. In social marketing this exchange is often less tangible and long-term, e.g. giving up smoking in exchange for a longer and healthier life. The product is hence more complex than buying a certain brand of cigarettes. The demand can also be more varied and target audiences harder to reach. The consumer involvement is more intense and finally the social marketer will have to compete with commercial marketers for the attention of the target audiences (Kotler et al., 2002).

\section{Price}

"Price" is what individuals/consumers have to do in exchange. If the price on a product is too high, fewer consumers will take action. In contrast to commercial marketing, the 'price' of healthier behaviours is often high. Accepting screening e.g. may be inconvenient, embarrassing, time consuming, or otherwise intractable. The 'product' may be right, but if the 'price' is not fewer will act. Although this type of exchange has no currency and is not adjustable by supply and demand mechanisms, 'prices' can nevertheless be lowered, e.g. if the screening offer is at a place and time convenient to the user. Six portions of fruit and vegetable may be even better than 
five ${ }^{1}$, but five could be more achievable, and so the 'price' must be adjustable to a given market. The traditional role of marketing, to create wants and to persuade consumers to buy even though the price seems high, is transferred in whatever way appropriate.

\section{Place}

The more the social marketer knows about the target audiences, who/where/when/what they do/what motivates them, the easier it is to design a health promotion campaign with the right mix of the 4Ps. Promotion teams will have to be creative and the places they advertise will have to be adjusted to the target audiences. If this concerns teenagers then another leaflet in the GP waiting room may not be as effective as a poster in a local shopping centre, for example.

\section{Promotion}

For social marketing campaigns to have any chance of success it will have to capture the audience, e.g. by appealing to emotions. The 'products' have to be advertised enticingly and be adjusted to both product and audience. In order to focus a campaign to a specific audience it will often be necessary to use qualitative research techniques such as observation, interviews, (multiple issues) group interviews or (single issue) focus groups (McCamley-Finney \& McFadden, 1999).

Social marketing smacks of manipulation and flouts several of the principles outlined in the section on principlism in chapter 1 . Nonetheless, some authors have justified it as 'countermanipulation' of healthy options to "commercial advertisers who influence the public's preferences by motivating a positive perception of unhealthy options" (Holland, 2007). This takes us back to the discussion of how campaigners will have to evaluate their work e.g. to the degree

\footnotetext{
${ }^{1}$ Refers to the 5-a-day campaign run by Department of Health in collaboration with the retail sector to encourage consumers to eat at least 5 portions of fruit or vegetable daily. In other countries the number of portions are 6 (Denmark) or 10 (Canada).
} 
they create autonomy (rather than respecting it), create positive freedom for the user as greater freedom to make choices in a long-term perspective.

\subsection{Conclusion}

The application of social marketing in public health campaigns is based on a premise that there are 'easy-win' endogenous behaviours in groups that given the right, motivational campaigning can influence the target audiences to voluntarily modify or abandon them. Social marketing uses similar terminology to marketing, although there are fundamental differences between the two. The so-called 4Ps of marketing - product, price, place and promotion - can nevertheless still be useful concepts for understanding how a social marketing campaign can be shaped in order to achieve health-promoting behavioural changes. The use of marketing techniques in public health campaigns falls close to manipulation and this potentially flouts ethical principles suggested for public health, i.e. the principle of least restrictive means (principlism, section 1.3). On the other hand social marketing can be justified on the grounds of being counter-manipulation against the over-powering commercial advertising for 'unhealthy' options. 


\section{Chapter 4 Public health targeting - a research agenda}

Having examined the concepts of health, public health as well as major political, economic and organisational factors influencing public health policy today, part one will here conclude with a research agenda for public health targeting. If we re-visit the objectives of social marketing as defined by Kotler et.al: "[to target audiences ] to voluntarily accept, reject, modify or abandon a behaviour" (Kotler \& Zaltman, 1971), we can use this to raise a number of essential questions. First, how do we select audiences for a given public health objective in ways which are scrupulous both ethically and scientifically? Second, to which degree does 'selection' also mean ‘identification' based on personal information? Third, to which degree can neighbourhoods, as characterised by geodemographic systems, qualify as target units?

On the first and second point together, more detailed information, especially concerning individuals and their behaviours over time, poses threats to information privacy and more fundamentally to civil rights. With regards to policy there are related concerns over whether interventions in actual fact reach those with the greatest needs (see e.g. Besley \& Kanbur, 1990; Clarke et al., 2008), which emphasises the importance of evaluating emerging methods for targeting of public health resources. Second, health is increasingly 'personalised', which e.g. is evident from the growing 'genomics' industry, where companies will offer customers a quickand-easy genetic screen for certain diseases (Katsanis et al., 2008). For some rarer hereditary diseases this can offer valuable reassurance, which is particularly relevant for compact ethnic groups, e.g. Ashkenazi Jews or Amish Christians (Gessen, 2007). Public health is however - as stated in section 1.3 - most clearly justified, where there are benefits for the public as a group, and where these benefits can only be obtained through collective rather than individual behaviour (Dawson \& Verweij, 2007 , p.27). Public health interventions are hence mainly directed at an 
asymptomatic population or at least addresses general behaviours that may be modified in the interest of the common good, e.g. drink-free driving or having your children immunized against infectious diseases. Where individual behaviours are targeted this type of campaigns are always voluntary, e.g. 'men's health' campaigns offering male employees of a company on-the-spot health screens and lifestyle advice. The division between campaigns targeting symptomatic individuals versus asymptomatic populations is not very clear, but the former is - especially if not strictly voluntary - problematic for two types of reasons. First, due to arguments for individual liberties (liberalism, section 1.3), and in addition with regards to the principles discussed for public health policy, e.g. least restrictive principle (principlism, section 1.3).

Second, it should also be useful to recall Coleman's principle about addressing 'mutable' factors in public policy (section 1.3). Genetic predisposition is individual, almost exclusively 'immutable', and in a public health context, the debate, at this point, easily risks sliding into the politically contentious area of eugenics (e.g. Gessen, 2007; Ridley, 2003). Eugenics is often attributed to Francis Galton (1822-1911), an industrious English scholar, who was convinced that humans could be bred much like domestic animals to improve certain physical ideals: 'health, energy, ability, manliness, and courteous disposition' (Michael, 2008). Set aside the imposition of identifying a universal set of such ideals, selective breeding programmes ironically narrow genetic variability and hence increase the risk for certain hereditary diseases (autosomal recessive disorders) due to founder effects and genetic drift (Ridley, 2003). Apart from the fact that eugenics would be grotesque and unethical on several accounts, it would, all else equal, be a very slow process of change. Genetic engineering and gene therapy may ease the lives of individuals with rare monogenic, hereditary diseases, but at present it is expensive and the results often unimpressive due to the bewilderingly complex associations between genotype and phenotype (Nebert et al., 2008). We may conclude with Ridley (2003) that 'nature' does matter and although it is no longer solely an ‘immutable’ (to use Colman's terminology in Aday and Andersen, 1974), 
'nurture', in contrast, remains the policy variable for public health - or to use the words of evolutionary biologist, Steve Jones (University College London), 'it is still better to send your

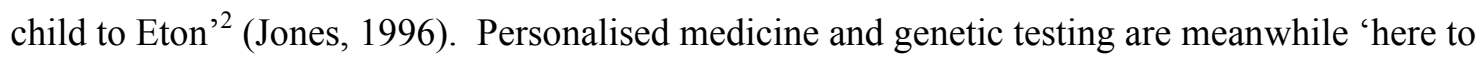
stay', but there are, as outlined above, several reasons for separating them from the definitions reached for public health (in section 1.3) as a field that aims to 'do good for the masses; without upsetting the interests of a single individual' through means of social change combined with individual agency. It is against this backcloth; methods to create evidence for public health policies, tackling 'mutable' upstream factors, will be developed and evaluated in the course of the thesis. Special attention will be given to methods to assess and monitor health behaviours and on this point - more specifically investigate the extent to which this type of interventions needs to be based on the exact identification of individuals or rather focus on classifications of aggregated data.

Third, the commercial sector has in the last few decades used geodemographic targeting for marketing purposes, i.e. the use of aggregated neighbourhood classifications based on consumer data; classically for distribution of targeted mail shots (also known as direct marketing) (Harris et al., 2005; Sleight, 2004). The Department of Health endorsed the medical data services company, Dr Foster's, work with geodemographic targeting of diabetes patients as an example of best practice in the White Paper "Choosing Health: making healthy choices easier" (Department of Health, 2004c). Yet there have been few independent studies of the validity of the claims made by commercial organisations about the cost-effectiveness of this practice. The promise of geodemographics is an easy and inexpensive way of targeting behaviours geographically, either through mail shots or by other means of social marketing that are used when health campaigns are

\footnotetext{
${ }^{2}$ In an interview comment with the Guardian newspaper on the feasibility of producing (genetically engineered) designer babies
} 
mounted in a local setting. The idea behind the use of geodemographics in marketing is simple. If we take a producer of, say, a new variety of biscuits. Maybe the producer has conducted some preliminary research with a panel of consumers and now plans a promotion campaign for its new product in its presumed geographical heartlands. As it is a new product the producer has no way of knowing in advance, where this product is likely to sell well, i.e. the producer has, in the terminology, only incomplete knowledge of the market. Maybe it emerges that the panel members showing the greatest interest in the biscuits also happened to be consumers of traditional products, say, Marmite ${ }^{\circledR}$ vitamin sandwich spread. Presumably nobody knows the exact market for this product either, but Marmite ${ }^{\circledR}$ does appear on the list of items covered by a larger consumer survey. By coding the postcodes of the respondents from this survey with a geodemographic system, it is subsequently possible to map the demand for Marmite ${ }^{\circledR}$ - and the new biscuits - across the whole country. Public health departments or other local health authorities are of course not in the business of selling sandwich spreads or biscuits, but would typically wish to promote messages for healthier living to particular target audiences. The process of selecting these audiences is a key topic for this thesis: who, where and how can these audiences be selected? Does geodemographics offer an easy and cheap way to target public resources efficiently? Does health promotion differ from commercial marketing in any way that requires the commercial techniques to be adapted to the needs of the new public 'masters'?

In the second part of this thesis the questions outlined above will be approached from different angles on the basis of studies undertaken in collaboration with NHS Southwark Primary Care Trust, London, UK.

Chapter 5 addresses the identification and information derived from personal names and names classifications. The first study concerns the identification of so-called 'inappropriate' users of an Accident and Emergency unit (A\&E). The second study covers the characterisation of nonresponders in a breast screening campaign. 
Chapter 6 concerns the techniques and policy implications for targeting organisations in public health campaigns with a teenage pregnancy campaign as an empirical example.

Chapter 7 addressed issues of construct validity for data models of areal aggregations, including geodemographioc systems, and their implications for the targeting of public health campaigns. Chapter 8 concerns the construction of neighbourhood classifications based on Census variables. Specifically, it demonstrates how a national Census classification can be modified to produce a regional classification and applied in the healthcare planning domain. This alternative classification is evaluated and compared to other popular neighbourhood segmentation/geodemographic systems. Two new performance criteria are proposed. Chapter 9 concerns the construction of a neighbourhood classification based on indicators of hospital demand for the most common long-term conditions. The merits of this alternative approach are discussed in relation to the general classifications for areal aggregations developed and described in chapter 7 and 8.

Chapter 10 concerns information privacy issues in the analysis of secondary data.

Finally, part three will provide a synopsis and account for the contributions to the social marketing agenda accrued in this thesis. 


\section{PART TWO: THE GEOGRAPHY OF SOCIAL}

\section{MARKETING}




\section{Chapter 5 Targeting individuals}

\subsection{Classifications of human difference}

In the first chapter we established the importance of autonomy, 'our capacity for rational selfgovernance', and the Kantian notion of human dignity, i.e. that we are 'uniquely and intrinsically valuable, and have no exchange value", in arguments concerning decisions balancing the interest of the individual against those of the group or society. It may therefore not be at all surprising that classifications of populations based on individual attributes, whether they are termed race, ethnicity, class, etc. have been and continue to be subject for both public and academic debate (Braun et al., 2007; Oppenheimer, 2001; Smedley et al., 2003).

Any classification can in fact be seen as human attempts to impose order on a chaotic world by choosing simplified representations based on selected criteria, which in turn may assist the observer in making decisions based on registration and analysis of phenomena of interest; be it of natural or artificial kinds (Longley et al., 2005). The last four lines do probably not stir much emotion with the scientifically inclined reader, but has in fact been at the heart of a fundamental discourse between proponents and critics of geographical information science ever since the discipline was defined in the early 1990s (Schuurman, 1999; , 2006). The processing of information itself is conditioned by a cascade of transformation processes going from the cognition (B) of an infinitely complex reality (A) to conceptual models (C) of the same. The third step, formalisation (D) crosses a virtual divide between social critics and proponents of information science; a divide between 'informal' and 'formal' model representations (Figure 2). The former would posit that the world is best thought of as 'unconquerable' by the forces of reason and that knowledge production 'unjustly' reinforces relations of power (Goss, 2003; Pickles, 1994; Schuurman, 2006). 
Figure 2 Conceptualisation and formalisation processes in information science
A) Reality
B) Cognition
C) Conceptualisation

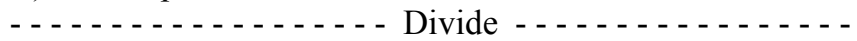
D) Formalisation
E) Digital analysis
F) Digital Representation

Adapted from Brodeur et al. (2003) and Longley et al. (2005)

Classifications are typical means of formalisation, but also a 'step too far' for the information technology critic that leads to an irreplaceable loss of information. Formalisation is, nonetheless, a critical step in information processing towards digitisation, data storage, transformation, retrieval, analysis and ultimately digital representations and evidence-based decision making (Longley et al., 2005; Schuurman, 2006). The response to the critique has been a focus on creating ontologies for geographical information science and quantitative geography; in the understanding that an ontology is “... a formal universe in which each entity is precisely defined and its relationship with every other entity in the specific categorical or computing realm is precisely determined. Ontologies in this context are the range of what is possible - in a computing context. They can be thought of as simply a classification system, a map legend, or a data dictionary" (Schuurman \& Leszczynski, 2008). Formalisation is in one way the Achilles' heel of information science and any classification should for reasons of reliability be used critically and not glibly adhered to due to its institutional credentials or perceived popularity (Douglas 1986 in Fullwiley, 2007).

Classification of human difference is - as hinted above - possibly one of the most contentious due to the historical use of the race concept to justify slavery, imperialism, anti-immigration policy, and a social status quo (Bhopal, 1997; Smedley \& Smedley, 2005; Smedley et al., 2003). 
Human classifications are nonetheless still used (and possibly also misused) in the political debate on topics ranging from the development of individualised drugs, mapping of hereditary diseases, issues of social mobility, equality, segregation, and migration (Fullwiley, 2007; Mateos, 2007b; Smedley \& Smedley, 2005). The question examined here is however will be confined to: under what circumstances classifications of individual attributes (biological, cultural or environmental) are justified and useful for public health targeting. In order to accomplish this, some frequently used definitions of race and ethnicity will in the following be examined in relation to differential health outcomes, the issues with current data availability to public health analysts and the potential of using classifications based on personal names as a proxy for factors of social and local nature.

\section{Race and ethnicity}

What is race? The race concept has mainly been based on differences in skin colour. The apartheid government in South Africa, for example, notoriously passed a Population Registration Act in 1950 defining three racial groups: coloured, white, and native. According to the act; "a 'coloured person' means a person who is not a white person, or a native... 'native' means a person who in fact is or is generally accepted as a member of any aboriginal race or tribe of Africa...'white person' means a person who in appearance obviously is, or who is generally accepted as a white person, but does not include a person who, although in appearance obviously a white person, is generally accepted as a coloured person" (Braun et al., 2007; Government Gazette, 1950). Many define race as a social construction with no foundation in biology; e.g. "[race is] a construct of human variability based on perceived differences in biology, physical appearance, and behaviour" (Smedley et al., 2003). Acceptance of race (in a biological sense) seems closely linked to racism and what Smedley and Smedley define as racial ideology, i.e. a set of factors that reinforce racial stereotypes. They also explain the characteristics of what they term the race-based society; 
1) Race classification - a classification of racial groups is observed and institutionalised.

These groups are seen as biologically discrete and exclusive. Physical characteristics

(e.g. skin colour, hair texture, etc.) are the 'inert' criteria for the classification

2) Race hierarchy - a biological determinism for differences and ranking of racial groups is maintained.

3) Race culture - cultural practices are perceived as inherited within a racial group (e.g. the existence of Black music or Black forms of dress)

4) Racial determinism - it is assumed that racial differences are innate and inherited and thus profound and unalterable. Racial segregation is encouraged if not enforced by law (adapted from Smedley \& Smedley, 2005, p.20).

Ethnicity has been suggested as a less contentious concept for public policy and health inequality studies. Smedley and Smedley describe ethnicity as purely social or cultural; something you could opt into or out of:

“... ethnic groups and ethnicity are not fixed, bounded entities; they are open, flexible, and subject to change, and they are usually self-assigned. Because culture traits are learned, ethnicity or ethnic traits are transmissible to other people - sometimes easily so, such as the widespread adoption of western dress (jeans and tee shirts) found all over the world, and the contemporary manifestation of industrial culture globally. History shows that people can and do learn another language and/or move into another ethnic group and become participants in that ethnicity." (Smedley \& Smedley, 2005, p.17)

Bulmer in contrast include biological traits such as ancestry and physical appearance;

"An ethnic group is a collectivity within a larger population having real or putative common ancestry, memories of a shared past, and a cultural focus upon one or more symbolic elements which define the group's identity, such as kinship, religion, language, shared territory, nationality or physical appearance. Members of an ethnic group are conscious of belonging to an ethnic group" (Bulmer, 1996).

Some argue that 'data disaggregated by race or ethnicity merely serves to create more social divisions and schisms and that the racial and ethnic disparities observed are generally the product 
of socioeconomic differences between the racial and ethnic groups' (cf. Smedley \& Smedley, 2005). Smedley and Smedley however conclude that:

“... race continues to play an important role in determining how individuals are treated, where they live, their employment opportunities, the quality of their healthcare, and whether individuals can fully participate in the social, political, and economic mainstream of American life. ....[race] is a means of creating and enforcing social order, a lens through which differential opportunity and inequality are structured" (Smedley \& Smedley, 2005, p.23).

Smedley and Smedley (2005) caution against abandoning race or ethnicity as concepts in public policy and argue that 'as long as governments fail to assess racial and ethnic inequality, racialized science will likely attempt to find explanations for racial hegemony in the biology and genetics of the "racial" group rather than in the social attitudes and institutions that perpetuate the idea of race' (Smedley \& Smedley, 2005).

Where does this leave us? The majority of population-wide health inequalities, warranting the attention of public health policy, are caused by behavioural and socio-economic factors and the biological heritage implied in the race concept is a falsity in that respect. The concept of ethnicity promises an alternative with its emphasis on non-biological aspects, although ethnicity in many cases seems to be used uncritically as a mere re-description of race (Sankar \& Cho, 2002). In the next section the question of race and ethnicity will be examined in the light of recent advances in genetic research. Is genetics a new beginning, a tabula rasa, or a cumbersome re-description of the race problem?

Genome-wide sequencing - a new beginning?

Recent advances in genetics have redefined the classification of human groups as genotypic combinations of nucleotide base pairs, genes, haplotypes, and chromosomes in which the complexity of the genome metaphorically becomes an unbreakable combination lock, i.e. almost unique and not easily classified into a narrowly defined range of categories (Sankar \& Cho, 
2002). While only a few diseases have been unravelled as simple combinations of genetic units to date, most seem to be coded in multiple loci and exhibit complicated (yet to be understood) interactions between genetic units, their expression and environmental factors. In the early stages of genetic research some anticipated a one-to-one causal relationship between single genes and their expressed phenotype, e.g. a gene for petal colour in pea flowers to use a classic example. Many traits (and propensity for certain diseases for that matter) are however inherited polygenically, i.e. dependent on the combination of several genes or variants of genetic units of different order between the nucleotide and the entire assemblage of the chromosomes. A further complicating factor is that the existence and expression of genetic units in one part of a genome may interact with the expression of units in other parts. The coding of the genomes are - beside 'simple' organisms such as bacteria - vast technological undertakings - and it has in this process been seen as essential by all means to filter down to the 'coding' fraction and leave aside the 'junk DNA' that many 'higher' organisms (including humans) seem to have accumulated on an evolutionary timescale. At present we know little about these so-called gene-gene interactions and in time 'junk DNA' may turn out to be important for a more complete understanding of genotype-phenotype relationship. The common diseases however that attracts the attention of the public health specialism, are almost exclusively polygenical - and in addition - responsive to a multitude of non-biological, social or behavioural risk factors (Tate \& Goldstein, 2004).

Another cautious point to consider before incorporating genetics and personalised medicine into to the public health domain is that the association between genotype (i.e. your genetic makeup) and phenotype (e.g. risk of developing a certain disease) poses an inverse problem in a double dose; the same genotype may lead to many different phenotypes - and the same phenotype may conversely be expressed from many different genotypes. Nebert et al. calls this the 'unequivocal genotype/phenotype problem' (2008).

The race concept, with its handful of categories mainly based on skin colour, seems absurd when considering the diverse possibilities in which an individual may react to a drug, or develop 
a complex disease (Braun et al., 2007; Fullwiley, 2007). A popular counter-argument for this position on racial medicine is the fact that the US government agency for drug approval, the Food and Drug Agency (FDA), in 2005 approved a race-specific drug, BiDil, for Black heart failure patients. It has later emerged that the initial trial only included patients that self-identified as African-American (Kahn, 2007). There seems to be evidence that BiDil works better than placebo for many heart failure patients, but the 'race' packaging does, at present, not seem well justified. Kahn speculates further whether race in this case has become part of a marketing ploy and a way pharmaceutical companies will try to justify recent investments in new genomic technology (Kahn, 2007).

Many diseases however have a genetic component and so-called genome-wide association studies have been initiated to search for genetic markers for diseases and to study whether drugs can be 'tailored' to patients based on DNA profiling techniques (Barreiro et al., 2008; Seng \& Seng, forthcoming). The challenge for genome-wide studies, the Human Genome Project, the HapMap I and II, and 1000-Genomes projects, has since the initiation of the first project in 1990 been to filter down to parts of the genome that are expressed, variable and thus 'meaningful' for differential health outcomes (Skipper, 2007; Spencer, 2008). The Human Genome Project established - after 13 years of immense international efforts - that $99.9 \%$ of genome was identical, i.e. it was $99.9 \%$ identical for the five unnamed Americans of different self-identified ethnicities they had sequenced (Seng \& Seng, forthcoming). The later HapMap projects were in turn concentrated on sites of Single Nucleotide Polymorphisms (SNP), a source of variation caused by harmless or at least non-fatal mutations in the human lineage, which can be used for disease profiling and 'fingerprinting'. The latest 1000-Genomes project aims to sequence a thousand individuals from around the world in the next two years at a rate of 8.2 billion nucleotide base pairs per day. Their press release explains: 
"With current approaches, researchers can search for two types of genetic variants related to disease. [1] The first type is very rare genetic variants that have a severe effect, such as the variants responsible for causing cystic fibrosis and Huntington's disease. To find these rare variants, which typically affect fewer than one in 1,000 people, researchers often must spend years on studies involving affected families. [2] However, most common diseases, such as diabetes and heart disease, are influenced by more common genetic variants. Most of these common variants have weak effects, perhaps increasing risk of a common condition by 25 percent or less. Recently, using a new approach known as a genome-wide association study, researchers have been able to search for these common variants. "Between these two types of genetic variants - very rare and fairly common - we have a significant gap in our knowledge. The 1000 Genomes Project is designed to fill that gap, which we anticipate will contain many important variants that are relevant to human health and disease" (Spencer, 2008).

Despite the new genetic framework, race is still used as a sampling frame in many studies and so - it is argued - reiterates the confusion over cause and effect in questions of race and health outcomes (Fullwiley, 2007; Sankar \& Cho, 2002). In order to gain more clarity for when 'race' may be warranted in genetic research, Sankar and Cho have outlined three different types of research questions. They state that race - in a biological sense - reflects population history, and may as such be warranted as a sampling frame where researchers require;

1) large-scale genomic variation to search for genetic markers of common diseases;

2) genetically similar subjects to identify genetic markers of rare diseases (association studies). They also state that race - in a social sense - is necessary as

3) a proxy for environmental exposures, including social interactions, in epidemiological research (Sankar \& Cho, 2002).

The human genome has so far been expensive and time-consuming to sequence in its entirety and to date only include a small number of 'prototypic' individuals. Research into genetic markers however is providing new assays for screening that could act as 'early warning' for patients with a hereditary disposition to e.g. inflammatory bowel cancer, prostate cancer, breast cancer and diabetes type I and II (McCarthy et al., 2008; Tate \& Goldstein, 2004). The preliminary results however show that the risk of developing the diseases, studied so far, is only $25 \%$ or (in most estimates much) less for those that screen as 'positives' compared to those with 'negative' 
screening results (Nebert et al., 2008; Spencer, 2008). Time will tell whether screening will be paid for by national healthcare systems under such odds or whether genotype screening will be a way for the pharmaceutical industry to profit on self-funding patients' fears.

Genetic markers are purely biological and are testament to population history. For epidemiological research, and hence public health policy, genetic markers risk becoming a redescription of the discredited race concept, contributing to racial ideology and, more importantly, missing out on pertinent problems concerning social inequalities (Fullwiley, 2007). Sankar and Cho however conclude that genetic markers may be valuable as proxies in a range of settings and that scientific journals should demand explanations for the degree to which selected markers are proxies for genetic, non-genetic or both types of effects (Sankar \& Cho, 2002). The problem with epidemiology (as opposed to, say, analyses of consumer or crime data) however is that 'nature and nuture' can never be fully separated. Montoya has - in his field of medical anthropology ${ }^{3}$ coined the term 'ethnorace' in acknowledgement of how ethnicity and race are intertwined in biomedicine. Race and ethnicity seem inseparable - confused in the way the terms are used. Many authors use them both descriptively and attributively interchangeably (Montoya, 2007):

\begin{abstract}
"Although ethnorace is essentially a social system of classification laid atop complex geographic patterns of population genetic variation, it is now possible to assign individuals to likely ethnoracial (or, sometimes, "ancestral") backgrounds via a cumulative examination of differences in the frequencies of common genetic variants as well as more geographically restricted (populationspecific) polymorphisms. Importantly, these efforts do not demonstrate that ethnoraces are naturally aggregating genetic clusters. Instead, they underline that individuals sampled from geographically disparate regions will often differ to a small degree genetically, and in a manner predicted by standard isolationby-distance models" (Montoya (2003) in Paradies et al., 2007).
\end{abstract}

\footnotetext{
${ }^{3}$ Medical anthropologists study the behaviour, interactions, reasoning and knowledge creation of biomedical research groups, their subjects and the wider contemporary political context of science
} 
In conclusion; the majority of population-wide health inequalities, warranting the attention of public health policy, are caused by social or socio-economic factors. Diseases themselves are however not just social phenomena or purely 'behavioural', but effects of interactions between both 'nature and nurture'. In this respect neither race nor ethnicity are fully adequate concepts; the former is too crude, the latter is ambiguously defined with regards to any biological effects. Genetic 'identity', as uncovered in new so-called genome-wide studies, is not easily categorised, but can be classified as clusters of genetic markers. For a small number of rare diseases there are clear genetic effects, but for most the hereditary component is small or as yet unknown.

Traditional race classifications are in this context of little practical or scientific importance.

Genetic polymorphisms of relevance to the prevention of specific health outcomes are however likely to play a significant role in individual medicine as new knowledge of genetic predisposition continues to unfold. Traditional race classifications are however still important for public policy and authorities have to continue recording and examining health inequalities, where these are the product of social processes falling along the lines of coarse-scale ethnic and racial (or 'ethnoracial') groupings.

The next section will describe some of the problems with self-identified ethnicity and examine how classifications based on personal names can be a way to profile local health inequalities.

\section{Names-based ethnicity classifications}

Classifications based on personal names have been suggested for health inequality studies across ethnic minorities for a number of reasons;

New classifications should address particular shortcomings in the current official ethnic classifications, e.g. used by NHS or in the Census, most importantly these concern:

1) Lack of reflectance upon the multi-dimensional nature of ethnicity

2) Scope for classification variability due to self-assignment of ethnic categories by users 
3) Self-assignment is restricted to a limited range of pre-defined categories

4) Missing and inconsistent data collection at registration or at points of contact

5) Limited scope for decomposition of the classification into subcategories (cf. Mateos, 2007b)

First, as established in the previous section ethnicity is a multi-dimensional and fluid concept that is not easily determined, but can be described using several indicators of identity and lifestyles including ancestry, birthplace, language, culture, and religion (McAuley et al., 1996). Coding a users name to geographical origin can, in this respect, be more informative than the self-assigned categories used at present, e.g. 'White other' (Mateos, 2007b). Second, studies have shown a considerable variation in the Census categories chosen by first generation immigrants from the same donor countries (Mateos, 2007b; Rankin \& Bhopal, 1999). Third, the Census classification has evolves over time to include categories for major ethnic groups present at the time of each census. New migrations can consequently be hidden in 'others' categories for up to a decade. A longitudinal study of UK Census returns compared the categories selected by responders in the 1991 versus 2001 Census (Mateos, 2007b; Platt et al., 2005). The study found that $23 \%$ of those that self-assigned to the Black and Black British categories in Census 2001 selected another group in the 1991 Census. The main explanation for this however was the introduction of new categories for mixed ethnicity in 2001 (White-and-Black African and White-and-Black Caribbean) (Platt et al., 2005). Fourth, there are two sources of ethnic data from routinely collected data; a) the patient register; b) data bases of healthcare usage, e.g. A\&E, hospital episodes, or breast cancer screening attendance. The former is usually not available for secondary analysis. For the latter data sources data are often missing or inconsistently collected, e.g. in some cases a user may identify as African Sierra Leonean, while others may state African or African Others resulting in coarsely aggregated categories with potential loss of ethnic information (Mateos, 2007b). Fifth, as a result of self-assignment and the use of coarse and sometimes overlapping categories the current classifications provide limited scope for decomposing ethnicity 
into subcategories. The names-based classifications are in contrast conveniently structured according to a rich hierarchy of geographical levels that may be aggregated or disaggregated to purpose.

As concluded in the previous section, classification of ethnicity (as encompassing both "nature and nurture' as defined by e.g. Bulmer, 1996) is justified for public health purposes, when it can give an epidemiological insight into local and specifically social determinants of health and healthcare utilisation. Except in the case of some rare diseases, there is no explicit need for any biologically determined indicators. The value of any particular ethnic classification increases with the degree to which it captures the spectrum of social factors relevant to chronic diseases with behavioural and modifiable risk factors. For population wide targeting based on routinely collected data, as discussed in this thesis, few individual data exist apart from sex, age and postcode. Occasionally self-assigned ethnic categories are available with the above mentioned limitations and inconsistencies. Name origin analysis been suggested as having 'the potential to provide embedded information about several ... dimensions of a person's origins, when no other ethnicity information is available, since names are usually unique to a language, a religion, a geographical area, a cultural tradition, a group of kin, a migration flow, etc. ... [ and can] offer a reliable method to ascribe individuals to common linguistic, geographic and ethnic origin' (Mateos, 2007b). The limitations quoted are the restricted number of names coded to ethnicity (or cultural, ethnic and language groups, CEL) and the partial spatio-temporal coverage of the reference population datasets used to produce name reference lists (Mateos, 2007b; Mateos et al., 2007). The former refers to the fact that none of the names-based ethnicity classifications are comprehensive, but are usually calibrated with expert knowledge to population studies within a given geographical region. In this they reflect the rich historic migration flows to a given host country, e.g. distinguishing secondary migration groups such as the British Asians born in East Africa (Gill et al., 2005). The latter refers to coding problems, where the reference list was calibrated with names from an earlier wave of migration, e.g. if immigration was initially from 
one region of, say, China - and the names-based classification with these names as 'Chinese' - it might fail to code a later wave from another region of China, because of the regional differences in the names material. Similarly a name might already be 'naturalised' at the stage of calibration and so fail to register new migrants from that geographical region; an example of this could be a name such as Landsman that in the UK electoral roll most frequently is associated with British nationality whereas the name probably originates in the Netherlands (see the departmental website for surname geography, www.publicprofiler.org/worldnames, accessed on 9 August 2008).

Name-based ethnicity classifications have been applied primarily in public health applications to subdivide populations into groups of common origin (Mateos, 2007b; Mateos et al., 2007; Nanchahal et al., 2001). The applications include a wide variety of areas including monitory of migration, census undercount, residential segregation, the geography of ethnic inequalities in health, evaluation of equal opportunity policies and political empowerment processes, and improving public and private service accessibility for ethnic minorities (Mateos, 2007a). Namesbased ethnicity classifications may in all of these areas provide a new edge, whereas before they were frequently hamstrung by the lack of appropriate, timely or detailed data on ethnicity. Improved methods for the measurement of ethnicity in these areas are thus of key policy importance in today's multi-cultural society (Mateos, 2007b).

\section{Ethnicity coding tools}

The ethnicity classification used in the example studies, Onomap, is a stand-alone software developed at UCL (Mateos et al., 2007). Onomap is a names-based ethnicity classification that is based on a technique, where the first names and surnames of a large sample of individuals are clustered according to the affinities of certain first names with certain surnames. These patterns are detected algorithmically and flagged as clusters in an iterative process. The clusters are subsequently coded with expert knowledge with respect to the specific cultural, ethnic and 
linguistic characteristics of each cluster. The resulting clusters are also known as CEL groups. The nomenclature for these typically refers to their geographical region of origin. The Onomap classification has eleven CEL Groups, which may be disaggregated into 185 CEL Types.

Birthplace is an important dimension of identity and indicator of ethnicity. It is however rarely available for secondary analysis, from routinely collected data sources in the healthcare sector, but was available for the empirical examples in this chapter through linkage with the local version of the patient register (also known as the GP register). There are however two main data issues with this source. First, the birthplace information is missing in many instances (see section 5.3, p.78). Second, birthplace is entered as free text, which usually means that its use is dependent on a very cumbersome data cleaning process. For the examples in this chapter the birthplace data however were cleaned and coded to geographical location with another new software, BirthPlaceGeocoder version 1.0. BirthPlaceGeocoder uses a worldwide gazetteer of place names that has been amended with birthplace information from patients registered in four inner city boroughs of London including Southwark and Lambeth (Mateos et al., 2005). Birthplace is in most instances coded to a country of origin and can thus be aggregated to any higher level of geography, e.g. the Caribbean or the Indian subcontinent.

Two empirical examples will in the following be used in an evaluation of names-based ethnicity classifications in a public health setting, viz. 'inappropriate' A\&E users and nonrespondents to breast screening invitations. The only consistent data available for the imputation of ethnicity were personal names in both case studies. Where self-assigned ethnic information was available it was only sporadically collected in the case of A\&E attendees or in the case of the breast screening campaign only collected for the women that attended the screening. Namesbased ethnicity classifications have recently been reviewed elsewhere (Mateos, 2007b) and rather than attempting a validation of the methodology itself, the focus of this chapter is instead on the potentials and limitations when these techniques are applied in a public health setting. 


\subsection{Using names to classify light A\&E Users}

Health authorities in many countries are trying to shift the balance of their interventions from acute towards preventative and planned care, wherever possible, in order to improve lives and cut costs (Bodenheimer et al., 2002; Lewis \& Dixon, 2004; Wagner, 1998; Wallace et al., 2006). Accident and emergency (A\&E) units are open 24 hours a day and are costly to run because of the number of specialised staff and the range of equipment needed at standby capacity. Users present with a wide range of conditions - some of which, although serious, may not warrant a specialist service. Complaints from staff about attendees 'that could have seen by their own GP instead' are therefore longstanding (Giesen et al., 2006; Murphy, 1998a; , 1998b; Sempere-Selva et al., 2001). Within London, Southwark and Lambeth Primary Care Trusts (PCTs) have each experienced a rise in the use of $\mathrm{A} \& \mathrm{E}$ and associated costs in recent years. This raises questions about whether it would be more cost-effective to divert users to other services such as General Practices (GPs), community care centres, or pharmacies. This study examines the characteristics of users of an inner city A\&E unit with regards to differences in A\&E outcomes, user age, neighbourhood deprivation, GP subscription and ethnicity as measured by a names-based ethnicity classification, Onomap (section 5.1) (Mateos et al., 2007). The principal questions are: who are the 'light' frequent users. What are their apparent needs and what alternatives could be offered to divert this group from using A\&E? More specifically we examine whether 'light' frequent $A \& E$ users are less likely to be registered with a GP or to be recent immigrants. The proposed approach can act as a 'first filter' to identify groups with particular needs or behaviours and so assist local health authorities in selecting e.g. focus groups (or other qualitative methods) and ultimately inform targeted interventions to reduce 'unnecessary' use of the emergency healthcare services. 


\section{Methods}

\section{Study area}

Southwark Primary Care Trust is situated on the south bank of River Thames in Central London. Historically Southwark was centrally located for London's port and associated industries. Today Southwark is among the most deprived local authorities in England; ranking 18th out of 325 local authorities on the income deprivation and 25th on the employment deprivation measures (Office for National Statistics, 2008a).

Table 1 Index of Multiple Deprivation 2004 profile of patients registered with a GP in Southwark PCT, April 2006. Mean IMD score is population-weighted

\begin{tabular}{lccc}
\hline Percentiles & Freq. & Percent & Mean IMD score \\
$1^{\text {st }}$ Most Deprived & 47,848 & 15.7 & 50 \\
$2^{\text {nd }}$ & 152,692 & 50.1 & 40 \\
$3^{\text {rd }}$ & 45,136 & 14.8 & 29 \\
$4^{\text {th }}$ & 30,764 & 10.1 & 24 \\
$5^{\text {th }}$ & 9,049 & 3.0 & 20 \\
$6^{\text {th }}$ & 12,765 & 4.2 & 16 \\
$7^{\text {th }}$ & 3,415 & 1.1 & 12 \\
$8^{\text {th }}$ & 2,835 & 0.9 & 10 \\
$9^{\text {th }}$ & 0 & 0.0 & \\
10 th Least Deprived & 0 & 0.0 & \\
Total & 304,504 & 100.0 & 36 \\
\hline
\end{tabular}

The majority of patients (66\%) live in areas ranked as the $20 \%$ most deprived areas in England (Table 1). More than $40 \%$ of patients live in publicly rented accommodation; typically social housing apartment blocks built in the 1960s on World War II bomb sites across the northern half of the Borough (Table 2).

Table 2 Tenure: percentage of households, April 2001 (ONS 2006)

\begin{tabular}{|c|c|c|c|}
\hline Tenure & Southwark & London & England and Wales \\
\hline Owner occupied: Owns outright & 9.7 & 22.1 & 29.5 \\
\hline Owner occupied: Owns with a mortgage or loan & 20.3 & 33.5 & 38.8 \\
\hline
\end{tabular}




\begin{tabular}{lccc}
\hline Rented from: Council (local authority) & $\mathbf{4 2 . 3}$ & 17.1 & 13.2 \\
$\begin{array}{l}\text { Rented from: Housing Association / Registered Social } \\
\text { Landlord }\end{array}$ & 11.2 & 9.1 & 6.0 \\
Rented from: Private landlord or letting agency & 12.5 & 14.3 & 8.7 \\
Rented from: Other & 2.6 & 2.9 & 3.2 \\
\hline
\end{tabular}

Southwark has relatively high unemployment, 6.2\%, against London, 4.4\%, and England and

Wales as a whole, 3.4\%. It also has a large student population (9.7\%) compared to London

$(6.6 \%)$ and England and Wales (4.7\%) (Table 3).

Table 3 Percentage of resident population aged 16 to 74 in each group, April 2001 (ONS 2006)

\begin{tabular}{lccc}
\hline Economically active/inactive & Southwark & London & $\begin{array}{c}\text { England and } \\
\text { Wales }\end{array}$ \\
Economically active: Employees Full-time & 41.1 & 42.6 & 40.6 \\
Economically active: Employees Part-time & 7.5 & 8.6 & 11.8 \\
Economically active: Self-employed & 7.5 & 9.0 & 8.3 \\
Economically active: Unemployed & 6.2 & 4.4 & 3.4 \\
Economically active: Full-time student & 3.6 & 3.0 & 2.6 \\
\hline Economically inactive: Retired & 8.0 & 9.8 & 13.6 \\
Economically inactive: Student & 9.7 & 6.6 & 4.7 \\
Economically inactive: Looking after home / family & 6.2 & 7.2 & 6.5 \\
Economically inactive: Permanently sick / disabled & 5.3 & 4.6 & 5.5 \\
Economically inactive: Other & 5.0 & 4.3 & 3.1 \\
\hline
\end{tabular}

Southwark has a very fluid, diverse and multicultural population with a large African and Caribbean communities (Table 4). Southwark scores low on a number of health indicators including infant mortality, low birth weight, low male life expectancy at birth, low disability-free life expectancy and high teenage conception rates.

Table 4 Ethnicity: percentage of resident population in each group, April 2001 (ONS 2006)

\begin{tabular}{lccc}
\hline Ethnic Group & Southwark & London & England and Wales \\
White & 63.0 & 71.2 & 91.3 \\
White; British & 52.2 & 59.8 & 87.5 \\
White; Irish & 3.1 & 3.1 & 1.2 \\
White; Other White & 7.7 & 8.3 & 2.6 \\
Mixed & 3.7 & 3.2 & 1.3 \\
Mixed; White and Black Caribbean & 1.4 & 1.0 & 0.5 \\
Mixed; White and Black African & 0.8 & 0.5 & 0.2 \\
Mixed; White and Asian & 0.6 & 0.8 & 0.4 \\
\hline
\end{tabular}




\begin{tabular}{lccc}
\hline Mixed; Other Mixed & 1.0 & 0.9 & 0.3 \\
Asian or Asian British & 4.1 & 12.1 & 4.4 \\
Asian or Asian British; Indian & 1.5 & 6.1 & 2.0 \\
Asian or Asian British; Pakistani & 0.5 & 2.0 & 1.4 \\
Asian or Asian British; Bangladeshi & 1.5 & 2.2 & 0.5 \\
Asian or Asian British; Other Asian & 0.6 & 1.9 & 0.5 \\
Black or Black British & 25.9 & 10.9 & 2.2 \\
Black or Black British; Caribbean & 8.0 & 4.8 & 1.1 \\
Black or Black British; African & 16.1 & 5.3 & 0.9 \\
Black or Black British; Other Black & 1.8 & 0.8 & 0.2 \\
Chinese or Other & 3.3 & 2.7 & 0.9 \\
Chinese or Other; Chinese & 1.8 & 1.1 & 0.4 \\
Chinese or Other; Other & 1.5 & 1.6 & 0.4 \\
\hline
\end{tabular}

\section{Data}

Records of all A\&E attendances at King's College Hospital, were obtained for a one year period (1 April 2005-31 March 2006). This facility principally serves the South London Boroughs of Lambeth and Southwark and the datasets were filtered to remove residents living outside of these two areas. This yielded a total of 141,613 attendances.

Most services in the National Health Service (NHS) record information on episodes of care labelled with unique person identifiers (NHS numbers). NHS numbers are issued when patients first register with a GP. However, NHS numbers are often missing from A\&E records, as users can 'walk in' from the street without an appointment, some are unconscious or distressed on arrival, and not everyone is registered with a GP. As a consequence, individuals were identified, in this study, by using a combination of date of birth, postcode, and sex in place of a unique identifier (Gill, 1999). We tested this method against the local GP patient register, where all users have a unique NHS number. This showed that the combination of these three fields correctly identified unique individuals in $99 \%$ of cases. Using this technique we identified 93,096 users from the 141,613 episodes collected over one year. 


\section{Deduplication}

Dealing with duplication of records within a file or across two or more files is one of the most time consuming tasks for analysts of secondary data. Deduplication is thus almost synonymous with the entire process of data cleaning (Winkler, 2006). There are several challenges or problems in this process. First, missing data ${ }^{4}$ constitute a big problem not least because of the costs associated with repeating data collection (e.g. in a Census) and if at all possible. Analysts will consequently have to find best possible matches for a given set of identifiers. Secondary data sets can internally or as assemblages over several files contain records that are asynchronous (Witten \& Frank, 2005), e.g. the same individual may in one data set be aged 47 but 46 or 48 in another data set. Discrepancies may also be caused by other upstream errors of recording or transcription (Gill, 1999). Gill (1999) usefully categorises identifiers according to whether they are:

a) Stable or even incorruptible - e.g. date and place of birth, date of death, sex, first name, initial, surname (with the exception of where it changes with marriage);

b) Transient or corruptible in nature - e.g. address (postcode), GP, marital status, occupation, ethnicity or other social categorisations;

c) Behaviours - e.g. medical records, such as past diagnoses and treatments, may be coidentifying; or

d) Lineage - variables used for family linkage studies, e.g. other surnames, parents' surnames, marital status, date of marriage, number of children, birth order, etc.

\footnotetext{
${ }^{4}$ as in incomplete collection of data for a variable where at least some data has been collected
} 


\begin{tabular}{|c|c|c|c|c|c|c|c|}
\hline & $\mathrm{FN}$ & SN & SEX & DOB & AGE3 & ONO_GR & $\mathrm{PC}$ \\
\hline $\mathrm{FN}$ & 14 & & & & & & \\
\hline $\mathrm{SN}$ & 85 & 24 & & & & & \\
\hline SEX & 11 & 20 & 0 & & & & \\
\hline DOB & 97 & 99 & 3 & 10 & & & \\
\hline AGE3 & 24 & 47 & 0 & 1 & 0 & & \\
\hline ONO_GR & 15 & 21 & 0 & 19 & 0 & 0 & \\
\hline $\mathrm{PC}$ & 86 & 52 & 1 & 99 & 11 & 4 & 2 \\
\hline $\mathrm{SEX}+\mathrm{DOB}$ & 99 & 100 & & & & & \\
\hline SEX + AGE3 & 35 & 70 & & & & & \\
\hline $\mathrm{SEX}+\mathrm{ONO}$ GR & 22 & 36 & & & & & \\
\hline $\mathrm{SEX}+\mathrm{PC}$ & 93 & 81 & & & & & \\
\hline $\mathrm{DOB}+\mathrm{AGE} 3$ & 99 & 99 & 19 & & & & \\
\hline $\mathrm{DOB}+\mathrm{ONO} \mathrm{GR}$ & 99 & 99 & 57 & & & & \\
\hline $\mathrm{DOB}+\mathrm{PC}$ & 100 & 100 & 100 & & & & \\
\hline $\mathrm{AGE3}+$ ONO_GR & 46 & 63 & 0 & 41 & & & \\
\hline $\mathrm{AGE} 3+\mathrm{PC}$ & 99 & 96 & 46 & 99 & & & \\
\hline ONO_GR + PC & 96 & 73 & 19 & 100 & 63 & & \\
\hline
\end{tabular}

Figure 3 Selection of identifying variables for repeated user based on match rates (\%) of patient attributes as a single variable (cells embounded in black) or in combination of two (upper half of diagram) or three variables (lower half of diagram). Sample source: Southwark patient register, 1 April 2006. Abbreviations: First name (FN), Surname (SN), Date of Birth (DOB), Age in 3-yr intervals (AGE3), names-based ethnicity, Onomap CEL Group category (ONO_GR), residential unit postcode of user (PC). The shading from dark to light in categories of increasing match rates: $<50 \%$ (dark grey), 50-74\%, 75-94\%, 95+\% (light grey).

The variables in the A\&E data were assessed by both their coverage (percentage of complete records) and general reliability for identification purposes following the categories stipulated by Gill above (1999). The local version of the GP patient register was used as a means of validation for these analyses (the date of extraction was 1 April 2006 and included all registered patients). The patient register is regularly updated and de-duplicated by the healthcare authorities. Every patient thus has a unique entry and a unique ID (the NHS number). The patient record is noticeably well covered with near $100 \%$ coverage across commonly collected identifiers such as name, residential unit postcode, sex, and date of birth. First, the number of unique records was calculated as a percentage of the total records (counting 319,251 patients) for each of these attribute variables in single 'dose' or in combinations of 2-3 variables. Identifying a minimum set of variables has an advantage, because the probability of a 'complete case' dwindles with the number of variables included. The following variables were included: patient's exact first name, 
surname, sex, date of birth, age (with a tolerance of $+/-1$ year), Onomap names-based ethnicity CEL group, and unit postcode (Figure 2).

Patients' surname was the most 'unique' single attribute, although this property was only present for $24 \%$ of patients; in other words, of all variables surname would on its own be the most specific variable for identification, because $24 \%$ of patients do not share surname with anyone else in the sample. In combinations of two variables, date of birth was a particularly strong coindicator of identity when combined with either first name, surname or unit postcode, yielding 97-99\% unique records. When three variables were combined personal name, sex, date of birth and unit postcode were the strongest contestants. Sex, date of birth and postcode were selected over personal names due to the general problem of typographical errors in names. Sex, date of birth and postcode yielded near $100 \%$ uniqueness in the records. Date of birth and unit postcode could have been used on their own $(99 \%)$, but because sex is very simple to code $(\mathrm{M} / \mathrm{F})$ it can be assumed that it is less prone to error and including it narrows the number of duplicates to a marginal minimum. So are there patients with the same sex, date of birth and unit postcode in the system at all? At closer inspection two likely causes of duplication within the data set were found; a) twins (or even multiple births) of the same sex also sharing the same address; and b) students in university accommodation. Many student halls operate a one-year-only policy and this creates a remarkably high turnover for a residential postcode. If we combine this with the fact that students represent a relatively narrow cohort at any one time this makes the co-occurrence of duplicates with the same sex, postcode and date of birth all the more likely. These exemptions accounted for less than $1 \%$ of all patients.

\section{Classification of outcome severity}

Many studies have been concerned with identifying A\&E usage that is deemed inappropriate, non-urgent, convenience-oriented, preventable or 'light' (Giesen et al., 2006; Murphy, 1998a; , 1998b; Sempere-Selva et al., 2001). In this research we have focussed on A\&E outcome 
categories, because in comparison with other attributes (diagnosis, triage, mode of transport) it is recorded at discharge rather than at arrival (cf. triage) and it was as a consequence recorded more comprehensively ( $86 \%$ of attendances) than for any of the alternatives. We reclassified the outcomes into four major categories of severity by drawing on the expertise of A\&E staff:

1. Majors containing major operations and emergency admission to hospital

2. Follow-up containing cases where the attendant are referred to another speciality

3. Discharged without follow-up; for those treated and discharged within 4 hours.

4. Did-Not-Wait for those that did not wait to receive attention.

Each user was subsequently identified by the outcome of their first attendance within the study period resulting in a user classification with Majors (16,865 or 16\% of all users), Follow-up $(26,202$ or $24 \%)$, Discharged $(47,645$ or $44 \%)$, Did-Not-Wait $(2,384$ or $2 \%)$, or Missing $(14,639$ or $14 \%$ ). To focus on users that potentially could be treated in other healthcare settings we have chosen to target repeated users, i.e. those that attended two or more times during the one year study period, in the Discharged and Did-Not-Wait category and termed these 'light' frequent users for the purpose of this study. In this way we identified 13,764 users (14.8\% of all users) with 38,002 attendances ( $28.8 \%$ of all attendances).

The first step towards receiving primary care is to register with a local GP. It is however difficult to assess the level of non-subscription or 'GP-lessness' in the whole population. Attendees to A\&E are asked about their GP details. GP-less A\&E users were identified as the users where the GP details were either missing or coded as unknown.

Ethnicity data were as is often the case with A\&E attendance data only sparsely recorded was and ethnicity was consequently imputed with the use of the Onomap names-based ethnicity classification Onomap (see section 0.0) (Mateos et al., 2007). 


\section{Statistical analysis}

Age- and sex-specific attendance rates were created for 'light' users and compared to the agespecific mortality rates published for London by the Office for National Statistics, ONS (Office for National Statistics, 2005).

Multiple logistic regression analysis was used to control for a number of potentially confounding factors with $p$ as the logit proportion of 'light' frequent users to all other users and $i$ denotes the respective age strata (Long \& Freese, 2006);

$$
\ln \left(\frac{p_{i}}{1-p_{i}}\right)=\beta_{0}+\beta_{1} x_{1, i}+\beta_{2} x_{2, i}+\ldots
$$

The following independent categorical variables, $x$, were included: sex, ethnicity and area deprivation derived from the 2004 Index of Multiple Deprivation (Department for Communities and Local Government, 2004). Their coefficients are denoted with $\beta$. There were significant interactions between age groups and ethnic groups, i.e. the age profile is generally younger for many ethnic groups relative to the majority population. Models were hence stratified by the following age groups: $0-15,16-29,30-44,45+$ years of age. Covariates were analysed for deviation from null with a Wald-test. The logit proportions of users not registered with a GP to all GP-registered users likewise analysed with sex, deprivation and ethnicity in age stratified analyses. 


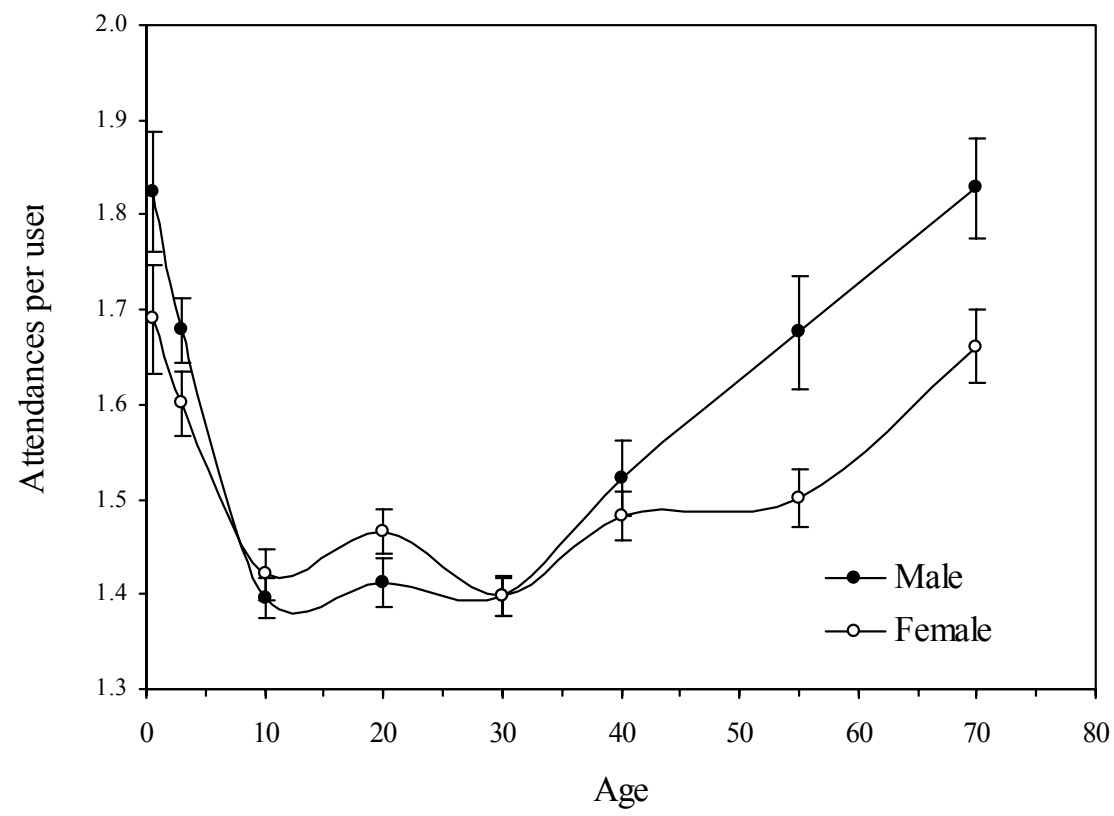

Figure 4 'Light' A\&E attendance rate by age

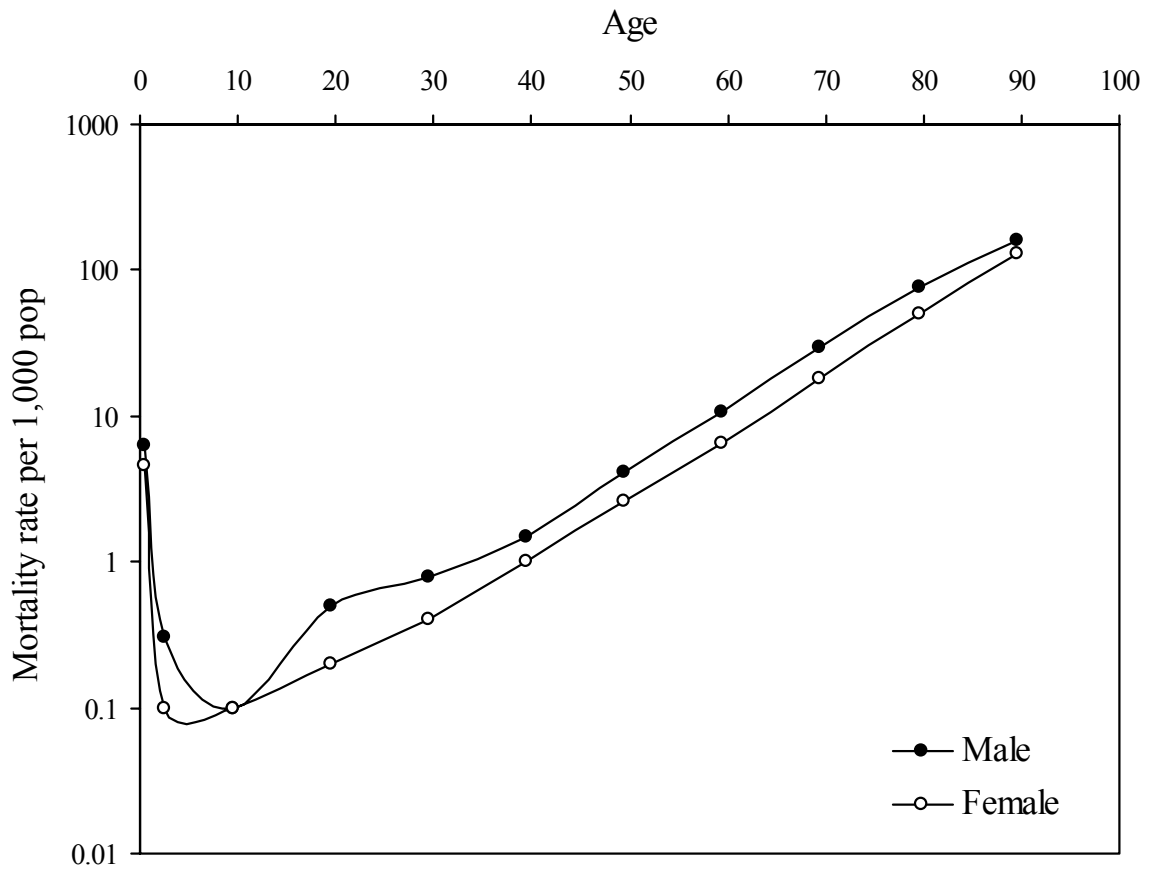

Figure 5 Mortality rate by age, London population 2000. Source: ONS (2005) 
Results

Attendance per user were highest amongst the under-1 and 1-4 year olds, but then dropped and plateaued only to rise again with age after 40 years of age for men and 50 for women (Figure 4). The higher rates for the under-5 year olds could be explained by the fact that this group is more prone to accidents and certain early childhood diseases, but we cannot in this study separate this from the so-called 'worried parent effect', where parents will take their young children to A\&E for reassurance to a greater extent than may be the case with older children. If we compare this to age-specific mortality we find an elevated risk early in life with a turning point after 1 year of age (Figure 5). This suggests that there is a need to consider actual risks alongside risks perceived by either parents or by healthcare professionals in understanding the higher attendance rates for infants. 
Table 5 'Light' frequent usage of $A \& E$. Multivariable logistic regression analysis stratified by age

\begin{tabular}{|c|c|c|c|c|}
\hline Age strata & Covariates & Level & Odds Ratio & P-value (Wald) \\
\hline \multirow[t]{19}{*}{$0-15 \mathrm{yr}$} & \multirow[t]{3}{*}{ Sex } & & & 0.3802 \\
\hline & & Men & Ref & \\
\hline & & Women & $0.97(0.91-1.04)$ & \\
\hline & \multirow[t]{6}{*}{ Deprivation } & & & $<0.0001$ \\
\hline & & 1st Least & Ref & \\
\hline & & $2^{\text {nd }}$ & $1.31(1.18-1.46)$ & \\
\hline & & $3^{\text {rd }}$ & $1.22(1.10-1.36)$ & \\
\hline & & $4^{\text {th }}$ & $1.25(1.13-1.39)$ & \\
\hline & & 5th Most & $1.22(1.11-1.36)$ & \\
\hline & \multirow[t]{9}{*}{ Ethnicity } & & & $<0.0001$ \\
\hline & & English & Ref & \\
\hline & & African & $0.96(0.86-1.07)$ & \\
\hline & & Celtic & $1.00(0.91-1.09)$ & \\
\hline & & Eastern European & $1.58(1.27-1.98)$ & \\
\hline & & European other & $1.11(0.93-1.32)$ & \\
\hline & & Hispanic & $1.17(1.03-1.35)$ & \\
\hline & & Muslim & $1.13(1.02-1.26)$ & \\
\hline & & Other & $1.18(1.06-1.31)$ & \\
\hline & \multicolumn{4}{|c|}{ Goodness-of-fit: $\mathrm{N}=24,363 ;$ Hosmer-Lemeshow $(\mathrm{df})=2.29(8) ;$ P-value $=0.9707$} \\
\hline Age strata & Covariates & Level & Odds Ratio & P-value (Wald) \\
\hline \multirow[t]{19}{*}{$16-29 \mathrm{yr}$} & Sex & & & 0.9885 \\
\hline & & Men & Ref & \\
\hline & & Women & $1.00(0.93-1.08)$ & \\
\hline & \multirow[t]{6}{*}{ Deprivation } & & & 0.0549 \\
\hline & & 1st Least & Ref & \\
\hline & & 2nd & $1.13(1.00-1.27)$ & \\
\hline & & $3 \mathrm{rd}$ & $1.15(1.02-1.29)$ & \\
\hline & & 4 th & $1.19(1.06-1.33)$ & \\
\hline & & 5th Most & $1.10(0.98-1.24)$ & \\
\hline & \multirow[t]{9}{*}{ Ethnicity } & & & 0.2873 \\
\hline & & English & Ref & \\
\hline & & African & $1.04(0.89-1.20)$ & \\
\hline & & Celtic & $1.00(0.98-1.24)$ & \\
\hline & & Eastern European & $1.01(0.84-1.20)$ & \\
\hline & & European other & $0.84(0.70-1.00)$ & \\
\hline & & Hispanic & $1.04(0.90-1.21)$ & \\
\hline & & Muslim & $1.08(0.95-1.23)$ & \\
\hline & & Other & $0.91(0.80-1.04)$ & \\
\hline & \multicolumn{4}{|c|}{ Goodness-of-fit: $\mathrm{N}=23,370 ;$ Hosmer-Lemeshow $(\mathrm{df})=3.82(8) ;$ P-value $=0.8727$} \\
\hline
\end{tabular}




\begin{tabular}{|c|c|c|c|c|}
\hline Age strata & Covariates & Level & Odds Ratio & P-value (Wald) \\
\hline \multirow[t]{19}{*}{$30-44 \mathrm{yr}$} & \multirow[t]{3}{*}{ Sex } & & & 0.0436 \\
\hline & & Men & Ref & \\
\hline & & Women & $0.92(0.85-1.00)$ & \\
\hline & \multirow[t]{6}{*}{ Deprivation } & & & 0.0007 \\
\hline & & 1st Least & Ref & \\
\hline & & 2nd & $1.02(0.90-1.16)$ & \\
\hline & & $3 \mathrm{rd}$ & $1.18(1.05-1.34)$ & \\
\hline & & 4 th & $1.19(1.05-1.34)$ & \\
\hline & & 5th Most & $1.23(1.09-1.39)$ & \\
\hline & \multirow[t]{9}{*}{ Ethnicity } & & & 0.2175 \\
\hline & & English & Ref & \\
\hline & & African & $0.91(0.79-1.06)$ & \\
\hline & & Celtic & $1.03(0.93-1.14)$ & \\
\hline & & Eastern European & $1.03(0.79-1.33)$ & \\
\hline & & European other & $0.84(0.69-1.03)$ & \\
\hline & & Hispanic & $1.07(0.92-1.25)$ & \\
\hline & & Muslim & $1.06(0.92-1.22)$ & \\
\hline & & Other & $0.89(0.76-1.04)$ & \\
\hline & \multicolumn{4}{|c|}{ Goodness-of-fit: $\mathrm{N}=22,787$; Hosmer-Lemeshow $(\mathrm{df})=14.7(8) ;$ P-value $=0.0653$} \\
\hline Age strata & Covariates & Level & Odds Ratio & P-value (Wald) \\
\hline \multirow[t]{19}{*}{$45+\mathrm{yr}$} & \multirow[t]{3}{*}{ Sex } & & & 0.0385 \\
\hline & & Men & Ref & \\
\hline & & Women & $0.92(0.84-1.00)$ & \\
\hline & \multirow[t]{6}{*}{ Deprivation } & & & 0.0002 \\
\hline & & 1st Least & Ref & \\
\hline & & 2nd & $1.19(1.05-1.36)$ & \\
\hline & & $3 \mathrm{rd}$ & $1.13(0.99-1.29)$ & \\
\hline & & 4 th & $1.29(1.13-1.46)$ & \\
\hline & & 5th Most & $1.32(1.16-1.50)$ & \\
\hline & \multirow[t]{9}{*}{ Ethnicity } & & & 0.6415 \\
\hline & & English & Ref & \\
\hline & & African & $1.13(0.93-1.36)$ & \\
\hline & & Celtic & $0.99(0.89-1.10)$ & \\
\hline & & Eastern European & $0.93(0.61-1.41)$ & \\
\hline & & European other & $1.00(0.78-1.30)$ & \\
\hline & & Hispanic & $1.21(0.98-1.49)$ & \\
\hline & & Muslim & $0.98(0.82-1.17)$ & \\
\hline & & Other & $0.98(0.82-1.17)$ & \\
\hline & \multicolumn{4}{|c|}{ Goodness-of-fit: $\mathrm{N}=22,576 ;$ Hosmer-Lemeshow $(\mathrm{df})=3.63(8) ;$ P-value $=0.8885$} \\
\hline
\end{tabular}




\section{'Light' frequent users}

The dataset was analysed for interactions between age and Onomap CEL Groups (Table 1). Age did vary significantly across ethnic categories (likelihood-ratio test, $\chi^{2}=41.33, \mathrm{df}=24$, $\mathrm{P}=0.015$ ) and the subsequent analyses were hence stratified by age group. More males attended than women and this gender effect was significant for the 30-44 and 45+ age groups (Table 5).

User's residence distance to the unit did not seem to play a significant role; in fact, attendance rates for $0-15$ yr olds were significant for the most distant tertiles. There were more 'light' frequent users in the more relative to less deprived areas, 29\% more among $0-15 \mathrm{yr}$ olds and $23 \%$ for $45+$ yr olds. Ethnicity was only significant as a covariate for the $0-15$ year cohort, where users with names classified as Eastern European and some other name minorities were more likely to be repeat users relative to the reference population with English names.

\section{Non-registration with a GP}

There were 15,062 A\&E users who were not classed as registered with a GP, amounting to $16 \%$ of users. Non-registration by age group was $6 \%$ for $0-15$ year olds, $30 \%$ for $16-29$ year olds, $19 \%$ for $30-44$ year olds and $9 \%$ for $45+$ year olds. Children in a combined assemblage of all ethnic minority groups had lower rates of registration with GPs relative to those with an English (the reference population) or a Celtic name (Table 6). Low rates of registration also persisted through all the adult age groups, especially for the Continental European and other minority name origin groups. Adult men were $39-59 \%$ less likely than women to be registered with a GP in their respective age groups. Area deprivation only showed an effect in the $45+$ yr group. Hypothetically users not registered with a GP might rely on A\&E for non-urgent care needs. However, we found that 'light' frequent users were consistently, 25-39\%, more likely to be registered relative to all other users. 
Table 6 GP registration. Multivariable logistic regression analysis of users not registered with a GP. Stratified by age

\begin{tabular}{|c|c|c|c|c|}
\hline Age strata & Covariates & Level & Odds Ratio & P-value (Wald) \\
\hline \multirow[t]{22}{*}{$0-15 \mathrm{yr}$} & \multirow[t]{3}{*}{ Sex } & & & 0.9582 \\
\hline & & Men & Ref & \\
\hline & & Women & $0.92(0.83-1.02)$ & \\
\hline & \multirow[t]{6}{*}{ Deprivation } & & & 0.1426 \\
\hline & & 1st Least & Ref & \\
\hline & & 2nd & $1.20(1.00-1.43)$ & \\
\hline & & $3 \mathrm{rd}$ & $1.14(0.97-1.36)$ & \\
\hline & & 4 th & $1.08(0.91-1.28)$ & \\
\hline & & 5th Most & $0.92(0.78-1.10)$ & \\
\hline & \multirow[t]{3}{*}{ User category } & & & $<0.0001$ \\
\hline & & Other & Ref & \\
\hline & & Light frequent & $0.61(0.53-0.71)$ & \\
\hline & \multirow[t]{9}{*}{ Ethnicity } & & & $<0.0001$ \\
\hline & & English & Ref & \\
\hline & & African & $1.23(1.02-1.48)$ & \\
\hline & & Celtic & $1.14(0.97-1.34)$ & \\
\hline & & Eastern European & $2.54(1.85-3.49)$ & \\
\hline & & European other & $1.52(1.16-2.00)$ & \\
\hline & & Hispanic & $1.82(1.48-2.24)$ & \\
\hline & & Muslim & $1.40(1.18-1.66)$ & \\
\hline & & Other & $1.53(1.29-1.81)$ & \\
\hline & \multicolumn{4}{|c|}{ Goodness-of-fit: $\mathrm{N}=24,363 ;$ Hosmer-Lemeshow $(\mathrm{df})=2.57(8) ;$ P-value $=0.9582$} \\
\hline Age strata & Covariates & Level & Odds Ratio & P-value (Wald) \\
\hline \multirow[t]{19}{*}{$16-29 \mathrm{yr}$} & \multirow[t]{3}{*}{ Sex } & & & $<0.0001$ \\
\hline & & Men & Ref & \\
\hline & & Women & $0.59(0.56-0.63)$ & \\
\hline & \multirow[t]{6}{*}{ Deprivation } & & & $<0.0001$ \\
\hline & & 1st Least & Ref & \\
\hline & & 2nd & $1.16(1.06-1.26)$ & \\
\hline & & $3 \mathrm{rd}$ & $0.90(0.82-0.99)$ & \\
\hline & & 4th & $0.91(0.83-1.00)$ & \\
\hline & & 5th Most & $0.85(0.78-0.94)$ & \\
\hline & \multirow[t]{3}{*}{ User category } & & & $<0.0001$ \\
\hline & & Other & Ref & \\
\hline & & Light frequent & $0.77(0.71-0.84)$ & \\
\hline & \multirow[t]{7}{*}{ Ethnicity } & & & $<0.0001$ \\
\hline & & English & Ref & \\
\hline & & African & $1.01(0.89-1.15)$ & \\
\hline & & Celtic & $1.00(0.92-1.09)$ & \\
\hline & & Eastern European & $5.26(4.61-6.00)$ & \\
\hline & & European other & $2.83(2.50-3.20)$ & \\
\hline & & Hispanic & $2.60(2.33-2.90)$ & \\
\hline
\end{tabular}


Muslim

Other
$1.13(1.02-1.26)$

$2.56(2.33-2.82)$

Goodness-of-fit: $\mathrm{N}=23,370$; Hosmer-Lemeshow $(\mathrm{df})=15.5(8)$; P-value $=0.0502$

\begin{tabular}{|c|c|c|c|c|}
\hline Age strata & Covariates & Level & Odds Ratio & P-value (Wald) \\
\hline \multirow[t]{22}{*}{$30-44$ yr } & \multirow[t]{3}{*}{ Sex } & & & $<0.0001$ \\
\hline & & Men & Ref & \\
\hline & & Women & $0.41(0.38-0.44)$ & \\
\hline & \multirow[t]{6}{*}{ Deprivation } & & & 0.0343 \\
\hline & & 1st Least & Ref & \\
\hline & & 2nd & $1.06(0.96-1.18)$ & \\
\hline & & $3 \mathrm{rd}$ & $0.96(0.86-1.07)$ & \\
\hline & & 4th & $0.96(0.86-1.07)$ & \\
\hline & & 5th Most & $0.89(0.81-1.00)$ & \\
\hline & \multirow[t]{3}{*}{ User category } & & & $<0.0001$ \\
\hline & & Other & Ref & \\
\hline & & Light frequent & $0.75(0.68-0.84)$ & \\
\hline & \multirow[t]{9}{*}{ Ethnicity } & & & $<0.0001$ \\
\hline & & English & Ref & \\
\hline & & African & $0.95(0.82-1.09)$ & \\
\hline & & Celtic & $0.92(0.83-1.02)$ & \\
\hline & & Eastern European & $3.30(2.74-3.98)$ & \\
\hline & & European other & $1.72(1.48-2.00)$ & \\
\hline & & Hispanic & $2.15(1.91-2.43)$ & \\
\hline & & Muslim & $1.20(1.06-1.36)$ & \\
\hline & & Other & $1.74(1.54-1.96)$ & \\
\hline & \multicolumn{4}{|c|}{ Goodness-of-fit: $\mathrm{N}=22,787$; Hosmer-Lemeshow $(\mathrm{df})=5.92(8) ; \mathrm{P}$-value $=0.6559$} \\
\hline Age strata & Covariates & Level & Odds Ratio & P-value (Wald) \\
\hline \multirow[t]{16}{*}{$45+\mathrm{yr}$} & Sex & & & $<0.0001$ \\
\hline & & Men & Ref & \\
\hline & & Women & $0.61(0.56-0.67)$ & \\
\hline & \multicolumn{4}{|l|}{ Deprivation } \\
\hline & & 1st Least & Ref & 0.0005 \\
\hline & & 2nd & $1.36(1.18-1.57)$ & \\
\hline & & $3 \mathrm{rd}$ & $1.23(1.07-1.43)$ & \\
\hline & & 4th & $1.18(1.02-1.37)$ & \\
\hline & & 5th Most & $1.10(0.95-1.28)$ & \\
\hline & \multirow[t]{3}{*}{ User category } & & & 0.0007 \\
\hline & & Other & Ref & \\
\hline & & Light frequent & $0.76(0.65-0.89)$ & \\
\hline & \multirow[t]{4}{*}{ Ethnicity } & & & $<0.0001$ \\
\hline & & English & Ref & \\
\hline & & African & $1.61(1.31-1.97)$ & \\
\hline & & Celtic & $1.14(1.00-1.28)$ & \\
\hline
\end{tabular}




$\begin{array}{ll}\text { Eastern European } & 4.45(3.30-6.00) \\ \text { European other } & 1.62(1.25-2.10) \\ \text { Hispanic } & 3.49(2.91-4.20) \\ \text { Muslim } & 1.56(1.29-1.88) \\ \text { Other } & 2.00(1.68-2.37)\end{array}$

A more detailed analyses at Onomap CEL Type level revealed that non-registration with a GP was much more common in users with names from countries within EU-15 as well as Eastern Europe and China (Table 7).

Table 7 Users not registered with a GP: Name origins (Onomap CEL Type) where more than 25\% of $A \& E$ users were not registered with a GP. Types with $<100$ users included in Other category.

\begin{tabular}{lcccc}
\hline Name origin (CEL Type) & GP & No GP & Total & Percent \\
\hline Poland & 701 & 723 & 1,424 & 50.8 \\
China & 185 & 140 & 325 & 43.1 \\
Russia & 126 & 56 & 182 & 30.8 \\
Greece & 151 & 64 & 215 & 29.8 \\
Germany & 335 & 140 & 475 & 29.5 \\
Spain & 1,779 & 679 & 2,458 & 27.6 \\
Italy & 1,335 & 497 & 1,832 & 27.1 \\
Hong Kong & 374 & 137 & 511 & 26.8 \\
Portugal & 1,952 & 713 & 2,665 & 26.8 \\
Albania & 113 & 39 & 152 & 25.7 \\
Norway & 217 & 74 & 291 & 25.4 \\
Balkan & 117 & 39 & 156 & 25.0 \\
Other & 36,169 & 6,700 & 43,180 & 15.5 \\
\hline England & 34,169 & 5,061 & 39,230 & 12.9 \\
\hline Total & 78,034 & 15,062 & 93,096 & 16.2 \\
\hline
\end{tabular}

\section{Discussion}

Children under 1 year of age were up to $80 \%$ more likely to be 'light' frequent users relative to the overall average. This result corresponds with the findings of Agran et al.: that children 1517 months old have the highest injury rates of all children under $16 \mathrm{yr}$ (Agran et al., 2003). This age period coincides with the time where most children start to stand up and learn to walk, develop inquisitive behaviour and increase hand-to-mouth activity. Agran et al. showed that the 
primary causes are falls, poisoning, transportation, foreign body, fires and burns in decreasing order. Another potential cause is the incidence of certain early age childhood diseases. Parents are generally thought to be more risk averse with their young children and will go to A\&E for reassurance. In this cross-sectional study it is difficult to separate out the 'worried parent' effect, but we can compare it with curves of age-specific mortality (Figure 4-5). Children under one year of age have an actual higher risk of mortality, a phenomenon sometimes referred to as biological mortality as it has persisted through the mortality transition even in developed countries (Gavrilov \& Gavrilova, 2001). This indicates that although children are the most frequent 'light' users, there are fundamental principles at work that would make it difficult to reduce this type health seeking behaviour.

More men than women fell into the target category of 'light' frequent users in the 30-44 and $45+$ age groups although only with a risk difference of 7-8\% (Table 6). The effects for area deprivation were stronger for $45+$ yr olds with $23 \%$ more target users in the more deprived areas relative to the less deprived areas. Area deprivation is a complex issue in itself, but may in this case, in addition to needs, indicate a greater desire for services that are 'convenient' and have a $24 \times 7$ availability.

Many studies of emergency healthcare services have focused on the needs of single ethnic minorities (Free et al., 1999; Naish, 1994; Weathers, 2004) yet rather fewer studies have sought to analyse the interactions between ethnicity and health-related behaviour in cross-sectional studies. Although there are important inequalities across ethnic groups for a number of health outcomes in the UK (Erens et al., 1999), 'light' frequent usage of A\&E does not seem to vary between them, at least for adult age groups. On the contrary, we identified very few significant differences within a very diverse user group with regards to this form of health seeking behaviour. The exception to this general trend concerns the $0-15$ year old age cohort, where users 
with names classified as Eastern European and some other name minorities were more likely to be repeat users relative to the reference population with English names. More qualitative research is needed to uncover whether these groups of families have particular needs that should be addressed and whether more could be done to accommodate these needs using primary care services.

The incidence of failure to register with a GP varied between users, with name origin analysis suggesting that ethnic minorities with a higher proportion of recent immigrant groups were up to 5.3 times less likely to be registered relative to the reference population with English names, even after controlling for area deprivation and age and gender differences (Table 6). Lower GP registration rates have for years been associated with the fluid populations of inner city areas, whereas rates generally are higher. Haynes et al. concluded that the GP register in many places are superior to the decennial Census as a source of demographic baseline data (Haynes et al., 1995). In this study the level of non-registration was particularly high amongst users with names originating in Poland ${ }^{5}$ (51\%) as well with users from many of the fifteen pre-2004 EU membership countries (EU-15) countries (25-30\% non-registration) (Table 7). This can be taken as support for the findings of Hargreaves et al. (2006) with respect to migrants arriving from specific worldwide refugee hotspots, but also suggests a need to pitch local campaigns for greater equity in GP-registration towards migrants from the EU (see Table 7). However, our results also suggest that a higher GP registration rate in itself is unlikely to reduce A\&E usage.

Although cultural, ethnic and linguistic name coding is still a recent innovation (Mateos, 2007b; Mateos et al., 2007; Nanchahal et al., 2001), our results suggest that it presents a very promising means of updating and focusing local healthcare programmes - such as those focusing upon improving equity in GP registration rates, for example. The already diverse composition of

\footnotetext{
${ }^{5}$ Poland is the largest of the ten new Eastern European countries that joined the EU in 2004
} 
London's population is changing rapidly and we cannot assume that ethnic minorities have uniformly low rates of registration, or indeed that recent migration or refugee status is the main risk factor. We suggest that our research establishes a need to update the specific and local evidence frequently, in order to establish precise local healthcare needs at any particular time.

\subsection{Using names to understand breast screening uptake}

Breast cancer is the most common cancer for women in the UK with over 44,000 new cases each year (Office for National Statistics, 2006a). Early detection has been shown to have marked effect in the reduction of breast cancer mortality for women aged over 50 years. The national target for screening of the $50-70$ year cohort is a $70 \%$ uptake. At this level it is estimated that breast cancer mortality could be reduced by $25 \%$. Uptake figures exceed the uptake target in most parts of the country, but have remained low in inner city areas including Southwark. Fewer than $57 \%$ of women in Southwark attended screening and more research is needed to address the causes of low uptake to find out whether the screening services could be made more effective.

This work concerns the relative strength of physical and cultural barriers for uptake of the more than 18,000 women, who were eligible for screening over a 3-year screening cycle (20032006 tax year). Typical of this and other secondary data studies contextual information have to be inferred from a handful of variables: date of birth, place of birth, personal name, residential postcode and the location of the breast screening clinic they were invited to. Names-based ethnicity is a relatively new and promising way to contextualise this type of analysis and is evaluated in a comparison using the birthplace information available for a part of the data set. Geographical access is generally included in analyses of screening uptake, because the equipment is often - as in this case -specialised, costly and needs to be operated by specialist staff. Health authorities therefore operate a network of clinics to which eligible women have to travel. In some cases - most pertinently in rural areas - women will have to travel long distances to be screened. This raises the question of what a reasonable distance is depending on the likely means of 
transport available to different individuals. Travel time by public transport was included in this study, because of the unusually low uptake figures and from the hypothetical viewpoint that this part of South London is underserved compared to North London, which generally has a much denser transport infrastructure. Car ownership, which would make up for lack of public transport, is generally lower in the inner city

\section{Methods}

\section{Data}

A total of 18,582 eligible women registered and resident in Southwark 2003-2006 (tax year) were included in this analysis. This excludes 61 women who were sent three invites during the campaign cycle and 1621 women with two invites constituting $8.3 \%$ of the total number of women. I decided to exclude them because, due to recording practices, it is not possible to tell the exact cause for this type of duplication; sometimes women self-request an additional screening or they postpone an appointment before a move of address and hence receive a new invite at the second address, etc. To avoid any bias from these unusual cases, e.g. from an increase in campaign efforts, women receiving just a one invitation has been used as sampling frame in the further analyses. Attribute data were merged on National Health Service ID to the breast screening records from the local patient register using an extract of the latter from April 2006, i.e. at the end of the three year screening cycle.

Personal first name and surname were coded using Onomap names-based ethnicity classification software, version 1.0 (Mateos et al., 2007). A small proportion of names could not be classified (184 names or less than 1\%). For the analyses the unclassified individuals were grouped together with low frequency Onomap CEL Groups in the class "Other".

The birthplace field were geocoded using BirthPlaceGeocoder software 1.0 (Mateos et al., 2005). For the eligible women in this study only 9,260 had birthplace information $(49.8 \%$ of total) and $94.2 \%$ of these were geocoded to a country-of-birth. 
Travel time between home and the clinic invited to was derived from the Transport for London's transport model, Capital. The model operates with a 100m grid resolution. The travel time estimates used in this study were the mean travel times from each of Southwark's some 800 Census output areas to every other output area (obtained on a commercial licence agreement between Southwark Primary Care Trust and Jacobs Consultancy, London).

\section{Statistical analysis}

Multiple logistic regression analysis was used to control for a number of potentially confounding factors. In the analysis of non-response, we used the logit proportion of this group relative to responders as the dependent variable (Long \& Freese, 2006). Due to incomplete recording of birthplace information ( $<50 \%$ of women gave birthplace information) and the associated unevenness by which women of different names-based ethnicity groups volunteer this information (Figure 6, Table 8), this attribute were not included in the overall logistic regression model, but more simply explored in a Mosaic plot (Friendly, 2001) with the tile size proportional 


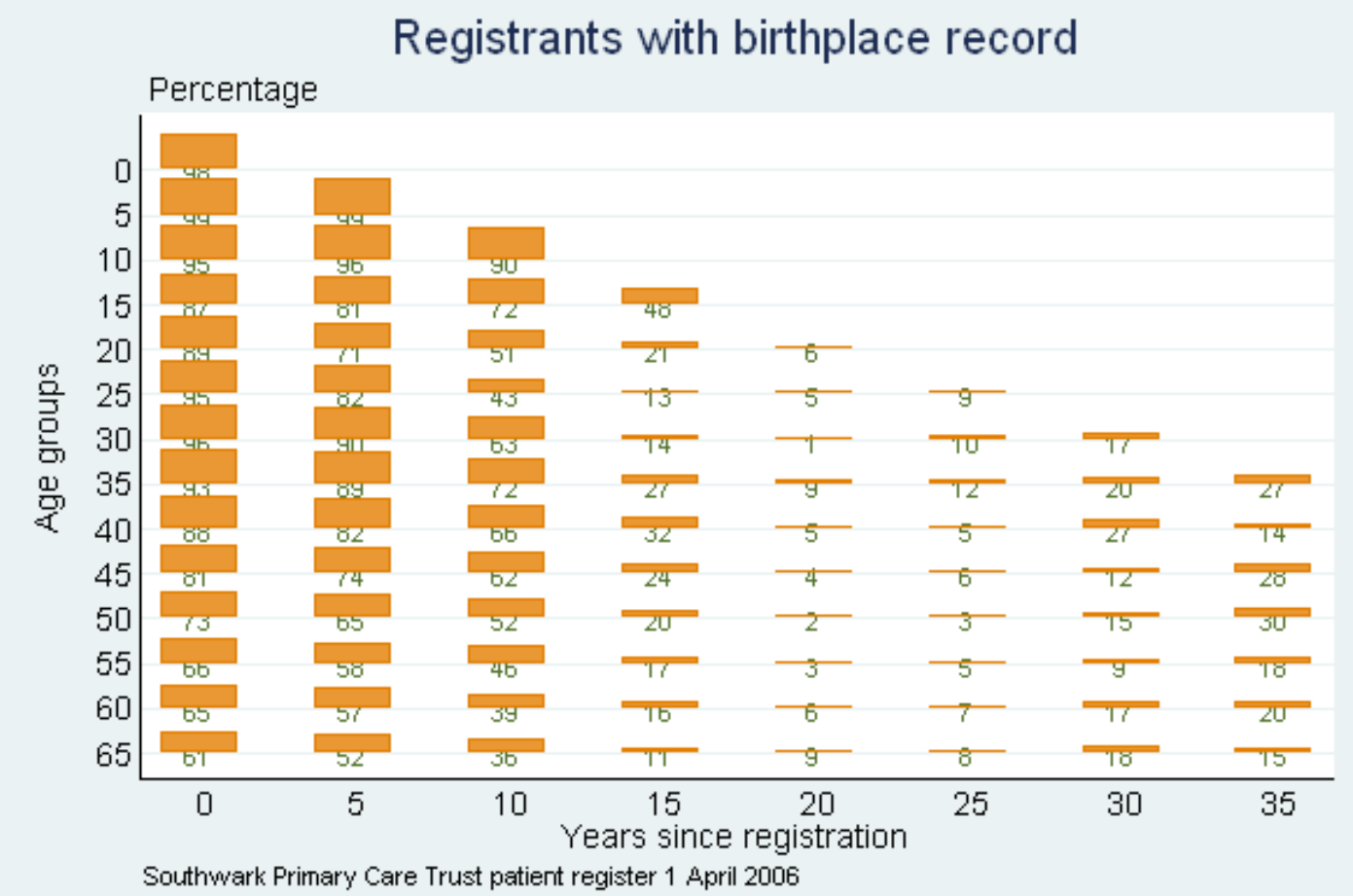

Figure 6 Percentage of registered patients with birthplace information in the patient register according to age and time since their registration. Southwark Primary Care Trust patient register, 1 April 2006. The bar in the top left hand corner is the percentage of 0-4 year olds that were registered within the previous 5 years (0-4 years from date of extract) and for which birthplace information were recorded. The bottom right bar is ditto for $65+$ year olds that registered for more than 35 years from date of extract, etc. 
Table 8 Inclination to give birthplace information across names-based ethnicity groups

\begin{tabular}{|c|c|c|c|}
\hline \multirow{2}{*}{$\begin{array}{l}\text { Onomap CEL Group } \\
\text { Number/Percentage }\end{array}$} & \multicolumn{2}{|c|}{ Birthplace Information? } & \multirow[b]{2}{*}{ Total } \\
\hline & Yes & No & \\
\hline \multirow[t]{2}{*}{ AFRICAN } & 1,287 & 340 & 1,627 \\
\hline & 79.1 & 20.9 & 100.0 \\
\hline \multirow[t]{2}{*}{ CELTIC } & 1,758 & 2,055 & 3,813 \\
\hline & 46.1 & 53.9 & 100.0 \\
\hline \multirow[t]{2}{*}{ EAST ASIAN } & 258 & 149 & 407 \\
\hline & 63.4 & 36.6 & 100.0 \\
\hline \multirow[t]{2}{*}{ ENGLISH } & 4,236 & 5,600 & 9,836 \\
\hline & 43.1 & 56.9 & 100.0 \\
\hline \multirow[t]{2}{*}{ EUROPEAN } & 282 & 175 & 457 \\
\hline & 61.7 & 38.3 & 100.0 \\
\hline \multirow[t]{2}{*}{ HISPANIC } & 269 & 180 & 449 \\
\hline & 59.9 & 40.1 & 100.0 \\
\hline \multirow[t]{2}{*}{ MUSLIM } & 748 & 480 & 1,228 \\
\hline & 60.9 & 39.1 & 100.0 \\
\hline \multirow[t]{2}{*}{ OTHER } & 265 & 235 & 500 \\
\hline & 53.0 & 47.0 & 100.0 \\
\hline \multirow[t]{2}{*}{ SOUTH ASIAN } & 157 & 108 & 265 \\
\hline & 59.3 & 40.8 & 100.0 \\
\hline Total Number & 9,260 & 9,322 & 18,582 \\
\hline Percentage & 49.8 & 50.2 & 100.0 \\
\hline
\end{tabular}

to the cross-tabulated number of women in each sub-category and the shading of tiles representing: high/middle/low uptake rates (the uptake rates in tertiles).

\section{Results}

The screening uptake was $56.9 \%$ with 10,573 respondents and 8,009 non-respondents in total. Multivariate logistic regression analysis showed that those who had to travel for more than 30 minutes on public transport were up to $20 \%$ less likely to attend screening (Table 9). While the main focus of this study is that of uptake, we should be aware of issues of coverage too. Are there 
women who are not accessing services because they are not registered with a GP in the area? To answer this question we have used the data set used in the previous section about A\&E users and extracted data on women aged between 50-70 years attending a local A\&E, King's College Hospital (2005-2006 tax yr), but who were not registered with a GP. If we assume that the women attending $\mathrm{A} \& \mathrm{E}$ are representative of the population, then the data show that $8.4 \%$ of women in this age group did not have a GP.

\section{Breast screening uptake by name origin and birthplace}

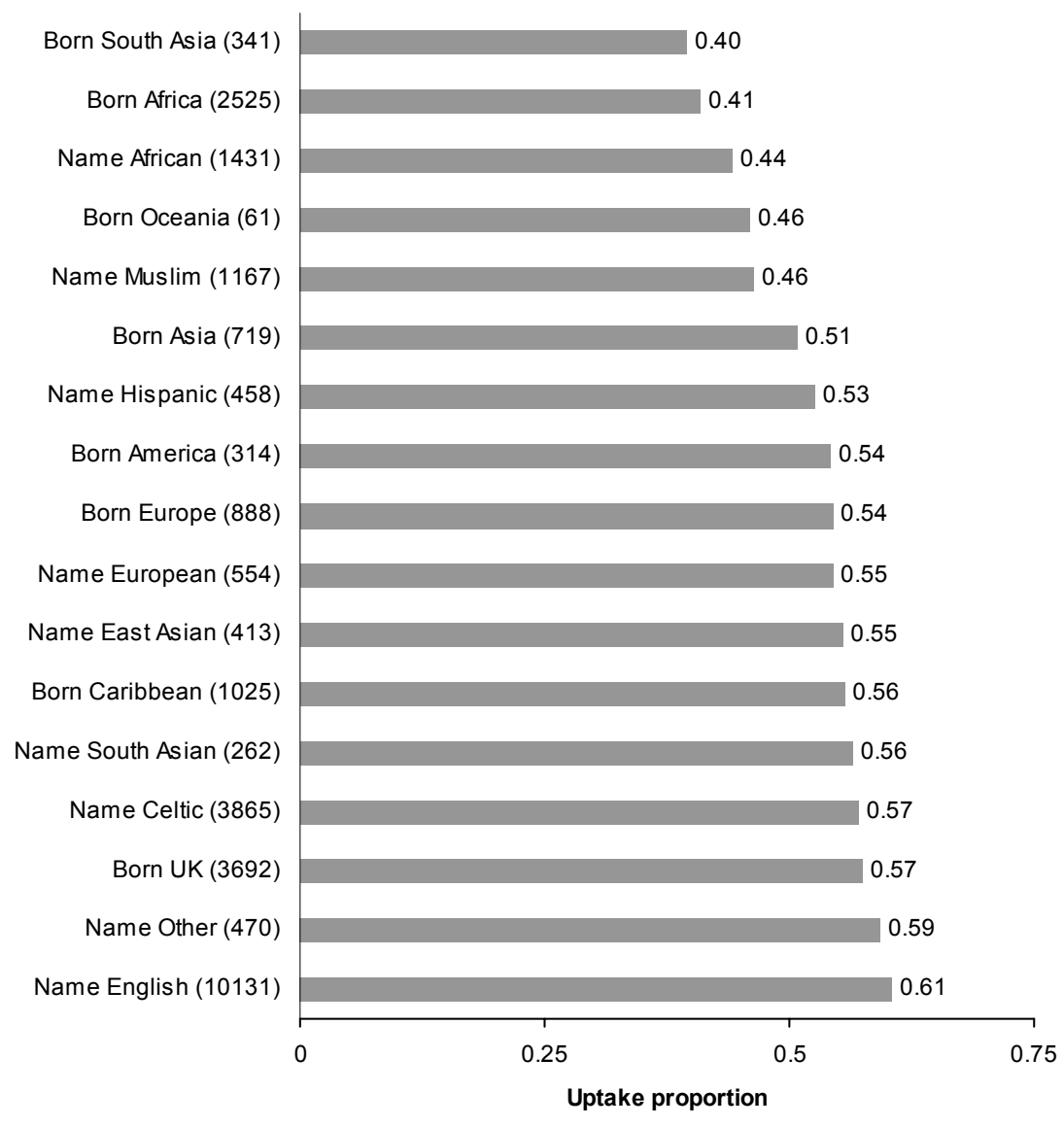

Figure 7 Breast screening uptake by names-based ethnicity and birthplace. The analysis of name origin is based on all women and the analysis of birthplace is based on the approx. $50 \%$ women for whom birthplace information is available. Numbers in brackets are the group frequencies.

Southwark PCT, 2003-2006. 


\section{Breast screening uptake by Name origin + Birthplace}

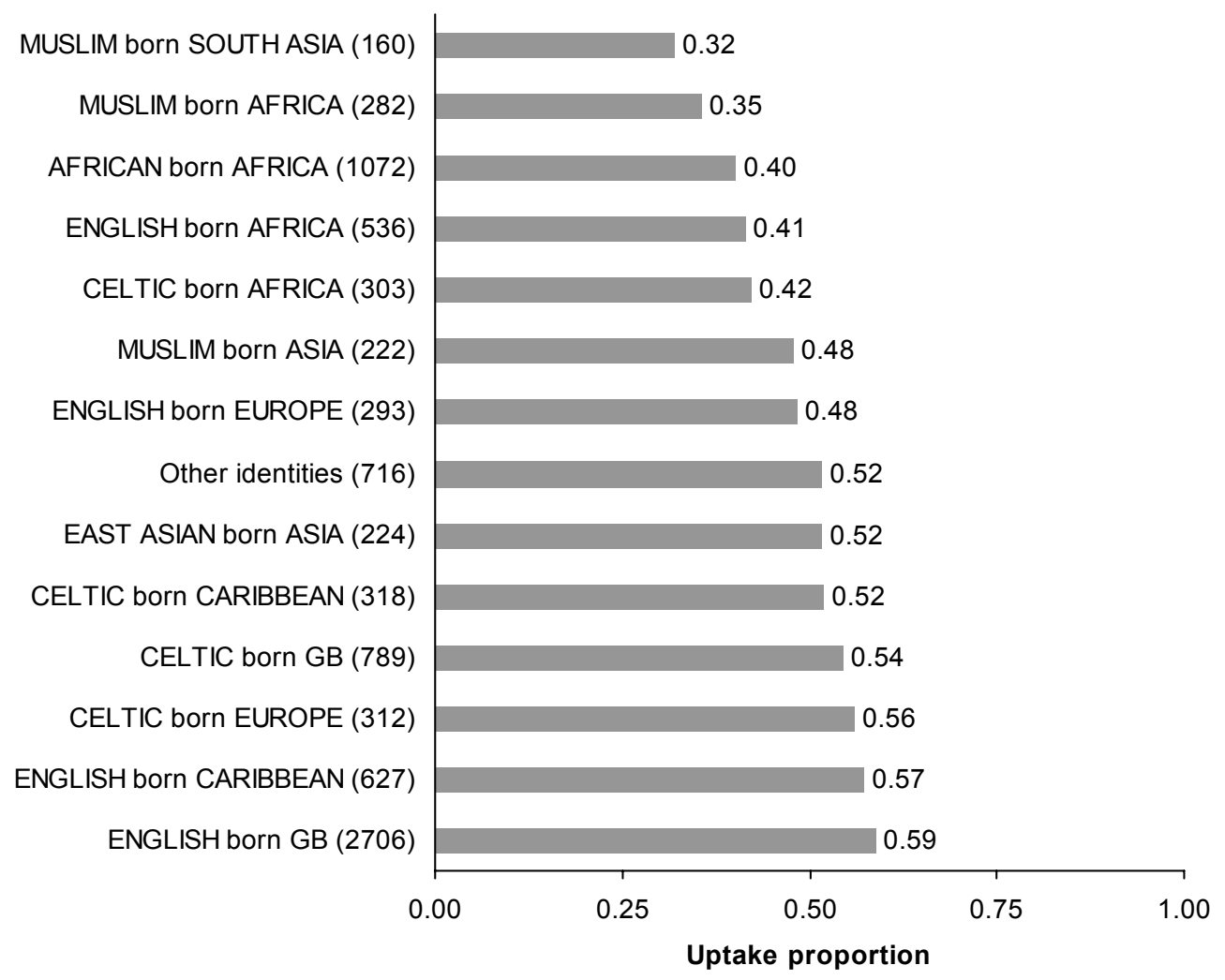

Figure 8 Breast screening uptake for name origin and birthplace combined. The combined analysis of name origin and birthplace is based on the approx. $50 \%$ of women for whom birthplace information is available. Southwark PCT, 2003-2006.

Using those with an English name as the reference population, most ethnic minority groups were less likely to attend. This was most pronounced in women with an African or Muslim name, who were up to twice as likely to miss screening (African odds ratio (OR) 2.13, 95\% c.1. 1.912.32. Muslim OR 1.89, 95\% c.1. 1.68-2.13).

Birthplace information was explored in cross-tabulations with names-based ethnicity. Because birthplace information is not available for all patients we have to be more cautious in interpreting the results, e.g. those with an African name were e.g. more likely to give birthplace information (79\%) than those with an English name (43\%) (Table 8). The cross-tabulation with names-based ethnicity revealed that women born in Africa were among the lowest third of uptakes almost 
regardless of the categorisation of their name. A Muslim name only seemed associated with the lowest uptake if the women were born in Africa or South Asia. Interestingly the women born in the Caribbean follow uptake patterns similar to women born in the UK (the host country), which could be interpreted as support for theories of integration and acculturalisation (Figure 7-Figure 9).

The recording of birthplace information in the local patient register also varied according to patient's age and time since registration with their present GP (Figure 6). Older patients were less likely to have filled out birthplace information, but the proportion with birthplace records in all age groups increased from less than $10 \%$ for 1980 s registrants to over $90 \%$ for the under-40 year olds and at least $54 \%$ for the oldest age group in the more recent figures.

Table 9 Non-response to Breast Screening. Multivariable logistic regression analysis

\begin{tabular}{llcc}
\hline Covariates & Level & Odds Ratio & P-value (Wald) \\
\hline Travel time & & Ref & 0.0004 \\
& $<19 \mathrm{~min}$ & $1.09(0.99-1.19)$ & \\
& $20-24 \mathrm{~min}$ & $1.10(1.00-1.20)$ & \\
& $25-29 \mathrm{~min}$ & $1.19(1.09-1.31)$ & \\
& $30-35 \mathrm{~min}$ & $1.20(1.10-1.32)$ & \\
& $36-58 \mathrm{~min}$ & & \\
Ethnicity & & Ref & \\
& & & \\
& English & $2.13(1.92-2.37)$ & \\
& African & $1.27(1.18-1.37)$ & \\
& Celtic & $1.21(0.99-1.48)$ & \\
& East Asian & $1.33(1.10-1.60)$ & \\
& European other & $1.42(1.18-1.72)$ & \\
& Hispanic & $1.89(1.68-2.13)$ & \\
& Muslim & $1.28(1.07-1.54)$ & \\
& Other & $1.32(1.03-1.69)$ & \\
& South Asian & & \\
Goodness-of-fit: $\mathrm{N}=18,582 ;$ Hosmer-Lemeshow $(\mathrm{df})=3.77(8) ;$ P-value $=0.8773$ \\
\end{tabular}




\section{Breast screening uptake, Southwark 2003-2006}

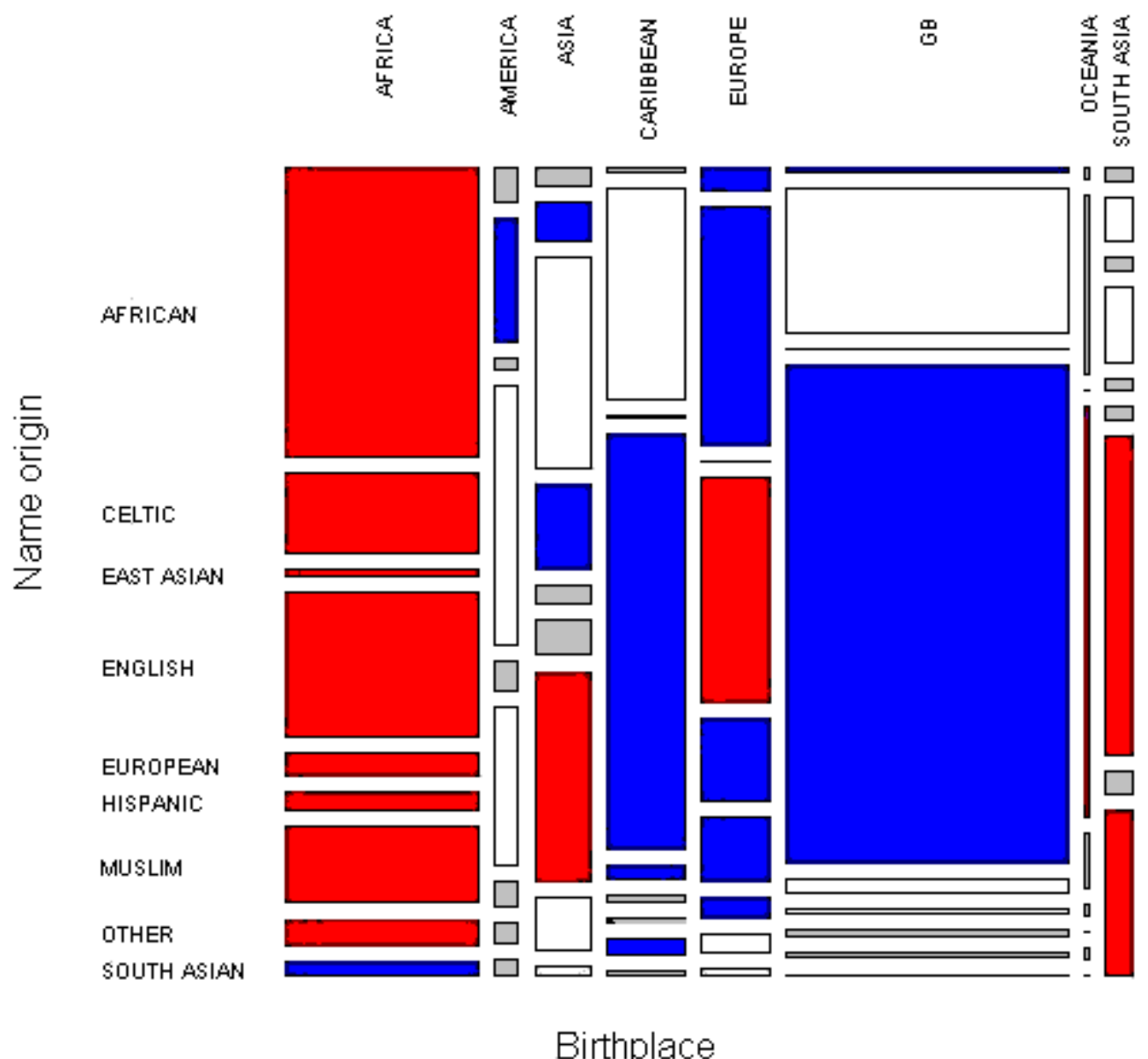

Birthplace

Figure 9 Breast screening uptake by names-based ethnicity and birthplace. The Mosaic plot shows the relative size of the different groups cross-tabulated for names-based ethnicity and birthplace. Women for whom birthplace information was not available (c. 50\%) were omitted from this analysis. The colour of the tiles in the Mosaic plot shows the uptake rate of each group in tertiles: High 56$73 \%$ uptake in BLUE, Middle 50-55\% uptake in WHITE, Low 32-49\% uptake in RED or insufficient data in GREY $(\mathrm{n}<20)$. NB The order of categories is constant, so that e.g. women with an English name is the fourth tile from the top in every birthplace column. 


\section{Discussion}

The low breast screening uptake in inner city areas is a good example where public health interventions may gain from a social marketing approach. How can breast screening be 'marketed' and if so to whom? What are the costs and benefits for women taking part in screening? What are the likely barriers? Is ethnicity coding from routinely collected databases an adequate tool for the evaluation and targeting of public health campaigns? The following begins with the potential benefits and costs of screening from a patient's perspective and then move onto a discussion about the wider barriers to increased uptake.

Research, including randomised control trials, during the 1980s and 1990s examined the detection limits for cancer tumours and established conventions for the grading of severities and growth stages. Patterns of tumour growth rates were studied and target age ranges were proposed on the basis of patient-centred cost-benefit analysis. Calculations from these studies showed that breast cancer mortality rates could be reduced nationally by $25 \%$ if clinics were able to screen at least $70 \%$ of women aged between $50-70$ years (Moss \& Blanks, 1998). Survival rates are steadily increasing, since the initiation of the national campaign in 1998; a fact that is attributed to the ongoing programmes for early detection and treatment of breast cancer. So, there seems to be great benefits in undergoing screening, but what are the costs? Financial costs are not negligible and will, from time to time, have to be reviewed strategically, i.e. whether the same funding could be used to improve health in other ways (Muir Gray, 1996). A full evaluation of financial costs however would be beyond the scope of this thesis, whereas the costs incurred for the patient, either physically or psychologically, will have direct bearings on the success of public health campaigns. The first question, in that vein, concerns healthcare in general; can you overtreat somebody? For many types of healthcare, including cancer radiotherapy, chemotherapy or surgery, benefits in terms of e.g. disability-free-years-of-life or increased utility of some sort, increases with the amount of healthcare received. This cost-benefit curve however might peak 
and show a slight net decline at the highest level of healthcare. This relates to the fact that any treatment or intervention will never be flawless and patients may suffer so-called iatrogenic effects (iatro- deriving from the Greek word for 'healer') comprising the risk of side-effects, being mis-diagnosed or receiving the wrong treatment, responding negatively to treatment or acquiring a malign hospital bug (e.g. MRSA), etc. Earlier cancer treatment had lower survival rates and one of the reasons for not screening women earlier is that the chances of 'doing harm' relative to 'doing good' has be shown to be much greater for younger women (recall the principles for healthcare discussed in part one; notably non-maleficence and beneficence). Apart from physical harm screening may also cause psychological harm to those that are mis-diagnosed. Diagnostic outcomes can be divided into whether the patient truly has a malign cancer; diseased $(+)$ versus non-diseased (-) in one dimension of a contingency table and whether the cancer is diagnosed at all; positive-tested (+) and negative-tested (-). This gives us four cases: true positive (diseased and diagnosed); true negative (non-diseased and correctly diagnosed so); false positive (not diseased but diagnosed as such); and false negative (diseased but cancer erroneously not diagnosed). The marginal effects of cancer screening and treatment are a central topic to cancer research, i.e. to improve the sensitivity and specificity of the treatment. Nevertheless some will still suffer the psychological effects of a mis-diagnosis; either because they for a period of time re-arranged their life in the belief that they had a terminal cancer or perhaps are coming to terms with thoughts about what they would have done, had they been correctly diagnosed earlier, etc. (for a more in-depth discussion of this area, see e.g. Gessen, 2007). These effects are difficult to communicate to the public without undermining the campaign aims themselves (Gigerenzer, 2002). The flip-side of this argument is, however, that if iatrogenic effects are not communicated properly, campaigns and interventions may as a result undermine patients' trust in their healthcare service as well as flouting important healthcare principles, e.g. about informed consent (see section 1.3). Problems reaching herd immunity for measles is an interesting recent example of public trust failure following the publication of a statistically flawed medical research study 
linking the measles vaccination programme (the triple measles/mumps/rubella vaccine) with autism in babies (Deer, 2006).

Table 10 Barriers to breast cancer screening among women from ethnic minorities. Adapted from Remennick (2006)

\author{
Structural barriers \\ Long distance to a screening facility \\ Lack of transportation \\ Inability to take time off from work \\ Lack of health insurance or GP registration (in the UK) \\ Inability to pay for child care \\ Organizational barriers \\ Difficulty understanding and navigating the healthcare system \\ Language barriers between women and providers \\ Arrogance or brusqueness of medical staff \\ Lack of female providers \\ Psychological barriers \\ Fear of cancer \\ Denial of susceptibility \\ Fatalism, often stemming from religious beliefs \\ Fear of and lack of knowledge about cancer treatment \\ Belief that treatment is futile \\ Sociocultural barriers \\ Complete dependence of women on men in some cultures \\ Subservient status of women in the family and the household \\ Possibility of being abandoned after a diagnosis of breast cancer
}

Both individual benefits and costs are of interest for the development of a social marketing approach to public health campaigns. The factors that potentially may act as barriers or facilitators for breast screening uptake will in the following be discussed in relation to the results of the analyses. The analysis showed that, holding travel time constant, women classified as having an African or Muslim name were up to twice as likely to miss screening as the reference population with an English name (African OR 2.13, 95\% c.1. 1.91-2.32. Muslim OR 1.89, 95\% c.1. 1.68-2.13. Table 9). The more refined analysis including birthplace showed that women born in Africa or South Asia were amongst those with the lowest uptakes. Women born in the Caribbean noticeably had equally high uptake figures to those of women with an English name 
born in the UK (see Figure 7 and Figure 9). Remennick (2006) has recently reviewed the literature on breast screening uptake among women from ethnic minorities and provides a lists of barriers subdivided into structural, organisational, psychological and socio-cultural categories (Table 10) that in the following will be used in a discussion of the findings.

Structural barriers pertain to whether women have the necessary resources and opportunities to access screening (Table 10) (for a more general discussion of healthcare accessibility see Aday \& Andersen, 1974; Joseph \& Phillips, 1984; Lovett et al., 2002 and section on neighbourhoods in chapter 7). Women in Southwark are invited to clinics on three different sites and if possible the screening authority normally invites the women to their nearest clinic. Even if the nearest clinic is not available, the longest possible journey would not exceed $10 \mathrm{~km}$. When we studied the actual journeys from residential postcode to the clinic to which they were invited we found that some would have to travel for up to $1 \mathrm{hr}$ on public transport. Including travel time in a multivariate logistic regression model also holding the women's names-based ethnicity we found an up to $20 \%$ lower propensity to attend for those that would have to travel more than 30 minutes (Table 9). This shows transport accessibility could be a significant factor to take in to consideration when planning of the location of new clinics in the future, e.g. by locating them on the main hospital sites, shopping centres or other transport hubs.

Women that are not registered with a GP will not get an invitation for screening. This size of this 'unknown' is hard to quantify with any certainty, but related to the previous case study in A\&E attendance, we know that $8.4 \%$ of women attending a local A\&E unit did not have a GP. This is a question of absolute coverage ${ }^{6}$ rather than of uptake, but does give us some idea of the

\footnotetext{
${ }^{6}$ The coverage term is usually used about the effectiveness of a campaign, i.e. the percentage of women screened relative to the total number of eligible women. Absolute coverage used here instead considers the likely underestimation of eligible residents, which may in part pertain to GP registration as a structural barrier in itself for some hard-to-reach groups.
} 
proportion of women that do not access screening because they have moved recently, are unaware of freely available healthcare, or for other individual reasons are not 'in the system'. Although it seems unavoidable that a proportion of women would at least temporarily not be registered, (absolute) coverage problems can potentially hide particularly vulnerable groups of women in ethnic minorities.

Organisational barriers concern the quality of healthcare services and the patient's experience of the service. For some minority groups there might be issues of language barriers or preferences for e.g. being treated by female staff. These are commonplace issues that seem well-embedded in the NHS-model, but might be subject for further and more qualitative research in this area. Szczepura (2005) refers to a number of organisational barriers that at least initially were an issue in the UK campaign including lack of knowledge, language barriers, inaccurate registers and lack of active encouragement from family doctors.

In addition to clinical factors, structural and organisational barriers, there are other more individual barriers. Psychological barriers relate to the individual's health beliefs and values ranging from fear of cancer, denial of susceptibility, and fatalism or lack of confidence in treatments that are available. Socio-cultural barriers relate to women's social status within the home or ethnic community. It is difficult to evaluate the significance or relative importance of these factors in this particular study, and they should be subject for more detailed and qualitative research locally. Do we for instance know whether information campaigns are 'working' in one sense, i.e. whether women have adequate knowledge about the cancer, the screening and the treatment, but - as Remennick suggests - there is a gap between knowledge and practice for many women (Remennick, 2006)? If there is such a 'gap' locally, it will be key to understanding the different motivational factors in order to improve breast screening uptake in Southwark.

Not all minority ethnic groups, as differentiated by names-based ethnicity and birthplace, were among the low uptake groups. The Caribbean born group is one of the largest immigrant groups in Southwark (see Appendix 1). This group had - as already mentioned - uptake rates similar to 
women with an English name born in the UK (Figure 9). Given that the main immigration wave from the Caribbean took place during the 1960s this result could be interpreted as one of acculturalisation. The longer one is resident in a host country, the more likely one is to adopt similar values and beliefs to the host population. Models of acculturalisation, the relations between host societies and immigrants, are subject to much research and political debate. The official policy in the UK is largely one of integration, viz. an 'accommodative approach in which host nationals believe that immigrants are entitled to preserve their heritage culture while simultaneously adopting aspects of the national culture. Those who endorse this strategy anticipate the gradual evolution of a multicultural society' (Van Oudenhoven et al., 2006). Another common model is assimilation, viz. 'a desire [from host nationals] to see immigrants relinquish their heritage culture in favor of the one from their adopted homeland'. Others again hold that national identity is a relative and social construct build on nostalgic values that are so wide-ranging, in the different lifestyles people can have, that it is impossible or unreasonable to stipulate exactly what national stereotype any immigrant should eventually 'assimilate' into (Van Oudenhoven et al., 2006). More qualitative research in this area is clearly needed in order to ensure that breast screening and other national health services are fully accommodating in areas that could be sensitive for ethnic minority groups that aspire to the same health values but have different norms in other areas, e.g. undressing in a hospital setting, etc.

\section{Conclusion - breast screening uptake study}

Few individual characteristics are collected routinely on users of healthcare services such as general breast screening examination campaigns. The present study has examined the value of coding names and birthplace information as two dimensions of identity or ethnicity that potentially could be used to target and improve uptake rates in a multi-cultural setting. It was estimated from $A \& E$ attendances that $8.4 \%$ of women in this area were not registered with a GP, which indicate the potential under-coverage in the area. The screening uptake percentage in 
Southwark was $57 \%$, a lot lower than the national target at $70 \%$ or the national average at $75 \%$. The effect of travel time and names-based ethnicity on uptake was estimated using a multivariate logistic regression design. Patients that would have to travel more than 30 minutes were up to $20 \%$ less likely to attend than those living nearest to the clinics. The effects of names-based ethnicity were even greater with women of African or Muslim names origin up to twice as likely not to attend relative to the reference population with English name origins.

Birthplace information was only given by approximately $50 \%$ of women in this age group when they registered with a GP, but there is a rising trend over time in the recording of this information. Names from ethnic groups other than English or Celtic were more likely to give birthplace information. The potential bias from missing data is estimated to be in favour in of low uptake groups among ethnic minorities. Cross-tabulations of names-based ethnicity and birthplace coded according to major groups showed that women born in Africa and South Asia were consistently among the lowest uptake groups.

Names-based ethnicity and birthplace coding were clearly valuable either as a sampling frame for qualitative research or to provide evidence for targeted strategies to improve breast screening uptake in a multicultural setting.

\subsection{Discussion: ethnicity classifications in public health targeting}

As set out in part one (chapter 4) three main questions are pursued in the course of this thesis; they are - in short - issues pertaining to target selection, user identification and the appropriateness of using neighbourhoods as target units. This chapter addresses the first of these questions and draws on empirical evidence from two studies, viz. 'light' frequent users of A\&E (section 5.2) and non-responders to breast screening invitations (section 5.3). Common to both studies is that they use a classification of personal names as an attribute for differentiation and target selection. Both of these studies can be categorised as health service equity analyses, although 'equity' here can have two slightly different meanings and present two different 
interests, i.e. supply versus demand side. In the case of non-respondents to breast screening invites the interests are primarily on the demand side; if it is assumed that it would be beneficial for the non-respondents, if more was known about them and that their needs could be accommodated when the campaign was redesigned, e.g. by better advertising or longer opening hours, etc. In return the local health authority (supply side) would appear more effective and possibly gain financial rewards for reaching certain target thresholds from central government. In the other case, regarding 'light' frequent users of A\&E, the interests are first of all on the supply side. Visits to existing GP practices or pharmacies are less costly to local health authorities than those to $A \& E$ and so the characterisation of the target group is motivated by cost cutting (supply side). It can however be argued that A\&E visits may not always be to the benefit of the user (demand side); in A\&E they are e.g. more likely to be treated by a trainee doctor or to wait a long time. Other binaries are that breast screening programmes are purely dealing with planned visits from registered patients, whilst the nature of $\mathrm{A} \& \mathrm{E}$ attendances means that visits are un-planned and its users frequently un-registered.

Why use a classification of personal names for health service equity questions? Chapter 7 will return to different ways of classifying areas for health needs mapping, but the following will briefly explain why the settings justify individual classification rather than area classification.

From a national or a regional perspective, the majority of inner city boroughs of London (including Southwark and Lambeth) stand out as 'hot spots' of concentrated needs for a range of health indicators from low birth weight to lower than average life expectancy (section 5.2). For many healthcare interventions local Primary Care Trusts (PCTs) (often coterminous with local authorities or in this case a London borough) represent the operational level, i.e. for the commissioning of primary (GPs and community care services) and secondary care (general hospital) services, public health campaigns, as well as auditing of their own services. Within this administrative level there is variation in needs that potentially could be used to differentiate and prioritise services further. In many cases tenure or housing categories are associated with e.g. a 
number of social factors and low income that potentially could be used for healthcare needs mapping. In the London Borough of Southwark 54\% of households are e.g. in the publicly rented apartment category (labelled "Council Flats" in the London Output Area Classification explained in chapter 7), which is very high for a local authority, but is related to the fact that Southwark until a few decades ago was centrally located for the London port industries and associated manual workforce before the industrial port was moved away from Central London in the 1960s. The concentration of needs is possibly also related to the shortage of 'affordable' homes in the London region and so the qualification criteria for publicly rented accommodation is such that only those with the absolute greatest needs, elderly singles, single parents, unemployed or minimum earners with dependents, would qualify for them.

In attempts to differentiate areas of particular needs in studies of health inequalities it was found that the place patients reside may not be sufficiently specific for targeting, the study of A\&E users only showed a significant effect of Indices of Multiple Deprivation (IMD) for the least affluent quintile of areas relative to the assemblage of the four more deprived quintiles together (more examples will be given later). In the breast screening study IMD was omitted from the model because it similarly only had weak effects. Initial analyses with geodemographic profiling did not produce any results suitable for targeting, because of the dominance of publicly rented accommodation in the study area, which are uniformly registered by the main geodemographic classification available in the study (chapter 7-8).

The application of names-based ethnicity and birthplace information in public health targeting seems more promising where disaggregated data are available, but this practice does simultaneously raise some issues that will be discussed in the following.

The prime justification for working with ethnicity coding from name and birthplace fields in database studies is the lack of systematically collected ethnicity data (Mateos, 2007b). The selfassigned ethnicity categories used often contain a high proportion of missing data. In the breast screening case self-assigned ethnicity was only available for those attending screening leaving the 
analyst without any comparable data for the non-attenders. In the A\&E case many users are not registered with a GP and so it was not possible to uncover additional information by linkage with other data sources; primarily the patient register (e.g. 30\% of 16-29 yr olds did not have a GP). All women invited to breast screening are, in contrast, already registered and so multiple ethnicity coding with both birthplace and names-based ethnicity could be explored for part of the data set. Birthplace has in itself been used as a single marker of migration, but as multicultural societies evolve (Van Oudenhoven et al., 2006), it loses its meaning with time as a marker of ethnicity and lifestyle. In the case of breast screening however birthplace still seems relevant; probably due to the fact that it concerns the 50-70 yr old cohort of patients, i.e. many may represent first generation of immigrants to UK for many different ethnic group (Gill et al., 2005).

The names-based ethnicity classification used in this study, Onomap version 1.0, was assessed as single proxy for ethnicity by comparing it to self-assigned categories in hospital admission data for Camden and Islington (Mateos, 2007a). The results showed that Onomap was sensitive in discerning names relative to their 2001 Census categories: White British (77\%), Pakistani (65\%), Bangladeshi (66\%), Chinese (63\%), White Irish (61\%), while it scored lower for Black African $(38 \%)$ and especially Black Caribbean (0.03\%). Members of the Black Caribbean group usually have English or Celtic names historically related to names of British plantation owners. It was noticeable that African born women were consistently among the lowest uptake groups for breast screening uptake almost regardless of the ethnic group they were imputed to on the basis of personal names. This finding illustrates the multi-layered nature of ethnicity and that classifications based on names do not always capture this complexity and so other dimensions of ethnicity should be used alongside this information if possible, e.g. birthplace. Where namesbased ethnicity displayed greater sensitivity, however, was to differentiate users of A\&E and GP registration in the A\&E study. This analysis showed that users with names typical of EU accession countries, e.g. Romania and Poland, were up to five times less likely to be registered with a GP relative to the patients with an English name. 
A much repeated syllogism in national media and by healthcare staff is that;

a) recent immigrants do not have a GP; and

b) people without a GP use A\&E more;

so, if $a$ is true and $b$ is true it must also be true that;

c) recent immigrants are more frequent users of $A \& E$.

This study could not support the notion that more recent immigrant groups would use emergency services for non-urgent visits and not the more established route of visiting a GP first. This example shows how names-based ethnicity can be a useful tool to test and analyse population trends in 'real time' - as it were - from local healthcare databases.

The measurement and analysis is not easily formalised, but may in large data sets of population data be useful as a first screen to discover patterns of health inequalities or differences in health behaviours whether culturally, socially or socio-economically induced.

Ethnicity as a proxy for lifestyles will be discussed further in the chapter on the use areal aggregations in health promotion campaigns (chapter 7).

\subsection{Conclusion}

The self-assigned ethnicity categories used in the Census and by the NHS are - when not meticulously collected - close to the race concept of distinguishing individuals mainly by skin colour and does not as such convey much sense of any social or cultural context for local health inequality studies. Names-based ethnicity is therefore promising for database studies of routinely collected data. A limitation is that names-based ethnicity classification only represents one of several dimensions in the construction of identity and ethnicity (Mateos, 2007b). McAuley et al. advocates for the present self-assigned ethnic categories to be replaced by a set of indicators comprising ancestry, birthplace, language, culture, religion and possibly also family structure and 
social position (McAuley et al., 1996). Cross-tabulating birthplace and names-based ethnicity arguably gave greater insight in determining factors in low breast screening uptake. One of the problems with multiple ethnicity categories however is of fragmentation. Already with two dimensions many groups become sparsely represented in local data sources and subsequently end up in unwieldy "Other" categories that in themselves can hide the very same vulnerable and 'hard-to-reach' groups that the ethnicity measures were intended to illuminate in the first instance. The aggregation of names-based ethnicity groups for the sake of statistical power have to be evaluated and balanced against loss of important 'ethnic' information.

In large national surveys and registers, multiple ethnicity classifications might be created using multivariate clustering techniques in a similar manner to those described for the creation of neighbourhood classifications in chapter 7 . But in a local setting this approach is very likely to replicate the fragmentation problem described above. A better approach for local health inequality analyses would be to explore health inequalities through the prism of a single or at most only in a few simultaneous dimensions in order to characterise the main and most clearly defined discriminating attributes of importance for any given aspect of health or healthcare utilisation. 


\section{Chapter 6 Targeting of organisations}

The policy response to rising trends in obesity, an array of disabling long term diseases in the population or simply any health outcomes that may reduce life chances in some way is focussed on the role of individual behaviour change with government White Papers entitled: "Choosing health: making healthier choices easier" or "Saving lives: our healthier nation" (Department of health, 1999; , 2004c). As discussed in part one some dimensions of ill health are correlated with macro-scale social arrangements and hence deemed harder to change, whereas individual agency in contrast appears at the same time powerful, constructive and enabling, 'cheap' and achievable. Behaviours that are shaped by social relations in particular, present 'easy-wins' for health modification programmes. This chapter looks at the theory as well as some practical ways in which local health authorities can a) create evidence about health outcomes from routinely collected data; and b) devise ways to make this evidence setting-specific. By setting-specific, is meant how stakeholders may be identified and targeted on the basis of evidence. A study regarding the reduction of teenage abortions, a key priority for Southwark Primary Care Trust,

will be used as an empirical example. Stakeholders are ultimately teenagers and their families, but as in many other areas of the public health specialism, interventions are multi-modal and the role of local organisations, public (schools, GP practices, etc.) or private (sports clubs, youth organisations) have to be acknowledged as the places fostering the intended behavioural changes whether these regard diet, exercise or sexual health. The example that will be developed at length here concerns sexual health. 


\subsection{Overview and problem definition}

Teenage pregnancy rates in UK are amongst the highest in Europe (Social Exclusion Unit, 1999). The Government's Teenage Pregnancy Strategy is designed to address this, aiming to reduce the number of teenage pregnancies and support teenagers more effectively (Social Exclusion Unit, 1999). There have been some signs of improvement; in the period 1998-2004 under-18 conception rates fell nationally by $11.6 \%$ from 47 to 42 conceptions per 1000 amongst the 15-17 yr old cohort (Office for National Statistics, 2006b). However, figures remained stubbornly high in many inner city deprived areas and the London Borough of Southwark (approximately 250,000 population) remains an area with one of the highest teenage pregnancy rates in UK. Here, the figures fell marginally from 87 to 85 conceptions per 1000 , or $2.3 \%$ of the age cohort, over the same period, and it is this Borough which is the focus of my study.

The Government's Teenage Pregnancy strategy strongly emphasises the need for a targeted approach (Social Exclusion Unit, 1999). However, local stakeholders and strategies are currently informed only by conception rates released by the Office for National Statistics (ONS) at Local Authority (average population of 300,000) or Census ward level (average population of 5,000). While this may be helpful in revealing a Local Authority such as Southwark to be one of the areas with the highest incidence in the UK, it does not present sufficiently detailed information to inform a targeted strategy at the local level. Ward level information can be said to represent local variation for many policy purposes, but because district boundaries are rarely coterminous with the principal catchment areas for e.g. schools or GP practices, they have proven less adequate for strategies targeting those organisations (Gibin et al., 2007; Glennerster, 1991; Harris \& Johnston, 2008). At present Primary Care Trusts and their local partner organisations ${ }^{7}$ are finding it difficult to target the most appropriate local areas and organisations in their campaigns.

\footnotetext{
${ }^{7}$ Local schools, colleges, youth clubs, GP practices, sexual health clinics and a broad range of community groups and non government organisations
} 
The National Health Service (NHS) is funded by the UK government and as such is central to the political debate not only about taxation, but also in questions of its share of the state budget in competition with other public sectors like education, policing, social security, defence, etc. (Hsiao $\&$ Heller, 2007). Within the healthcare system itself, there are complicated trade-offs between equity and efficiency objectives with respect to improving population health, reducing risks and inequalities, and a need to ration services balanced with maintaining a certain level of user satisfaction (Musgrove, 2003). There is consequently increasing pressure to reform healthcare systems and the NHS is undergoing reforms to make its organisation more cost-effective and to attract private enterprise (Pollock et al., 2007; Talbot-Smith \& Pollock, 2006). More resources are now directed towards local health authorities with emphasis on primary and community healthcare. Additionally, GP practices are rewarded for matching services to local needs in their recently renewed contracts with the Department of Health (Department of Health, 2006). The urge for public health decisions to be based on evidence and provide value-for-money is a ubiquitous demand and central to the healthcare reforms (Department of Health, 2000; , 2006; Muir Gray, 1996). While it seems clear that economic gains should accrue from different forms of optimisation and resource targeting in sexual health campaigns, this does also raise some critical issues concerning individual rights to privacy and data protection.

With this chapter, a new approach for a targeted strategy at a local level, using fine-scale conception data from both local hospitals and private abortion clinics, is proposed. It describes and demonstrates some simple methods that make it possible to estimate risks for areas, schools and GP practices, while remaining cognisant of confidentiality strictures and the practical requirements of ethical approval procedures. Local health authorities will, using the proposed methods, be able to target their limited resources more effectively and base campaigns on local evidence and in line with the sector reforms described above. In what follows a method for ascribing information on unwanted pregnancies to localities using visual communication that is readily intelligible to a range of stakeholders. As such, the method can be seen as providing a 
valuable 'first filter' to preventive healthcare campaigns, that is applicable in the widest range of settings and which can provide a framework to specific locality studies. The findings are discussed with regard to geographical targeting, data confidentiality, multi-agency strategies and resource allocation in a local healthcare setting. The proposed method is by no means a complete solution to targeting initiatives, but rather as a contribution to the more effective use of routinely collected local data as a kind of 'first filter' for locality studies of healthcare needs. As such this may offer an important contribution to increasing cost effectiveness and improving intersectional collaboration without infringing the privacy rights of any individual or storing sensitive data material on any identifiable individuals.

\subsection{Methods}

In order to address the problems of disaggregating official records in a way that could inform local strategies to reduce teenage pregnancy, Southwark Primary Care Trust collected detailed teenage conception data including all recorded maternity and legal abortions from all NHS service providers and private providers commissioned by the trust during the period 2002-2005 (Butt et al., 2006). This data assembly exercise identified 885 conceptions locally coded with residential unit postcodes, which equates to $86 \%$ of the conceptions reported by the Office for National Statistics (ONS) over the same period. It is difficult to identify the exact causes of the apparent shortfall, but one possibility is likely to be users paying a private provider themselves. In these cases ONS would be notified, but no claim for payment would be made to the users' PCT. Under-18 year denominators for unit postcodes were derived from the Mosaic (Experian Ltd., Nottingham, UK) directory. Other population data obtained included anonymous postcoded records of resident 11-17 year old girls attending either a state secondary school or registered with a GP in the trust. The former were obtained with the consent of the Local Educational Authority. Ethical approval to analyse the data sets containing patients' postcodes was obtained in accordance with NHS ethical guidelines from the Local Research Ethical Committee (Department 
of Health, 2003). Data analysis was carried out using Stata 9.2 (StataCorp, College Station, USA), ArcGIS 9.1 (ESRI, Redlands, USA) with the Hawth's Tool extension (Beyer, 2004), and the DCluster disease cluster package for R (Gomez-Rubio et al., 2005).

\subsection{Results}

Persistent inequalities associated with place have causes rooted in a matrix of compositional and contextual factors, with potentially self-enforcing ties between the two (Cummins et al., 2007). For purposes of local policy, identification of spaces exacerbating 'risk' is therefore important. However, the relational view of place in health inequality also leads us to think about how the knowledge of flows from residence to schools or GP practices could be utilised to inform local strategies. For purposes of the further empirical analyses, 'risk' is approached in three different ways: (a) areas with the highest densities of teenage conceptions; (b) areas with the highest densities adjusted for the underlying population; and (c) school or GP practices where risk is particularly high. The term 'risk' is in other words following a purely frequentist approach (Waller \& Gotway, 2004 p.9), where risk is evaluated numerically as the number of cases relative to either space (as in (a) above), population aged under 18 years (as in (b) above) or genderspecific school populations (as in (c)). In each case mapping and data analysis can be used to inform public health campaigns about the most appropriate areas, populations and organisations to engage in a range of targeted strategies - including the location of advertising boards, routing of the public health information bus and the involvement of GP practices, schools and youth clubs. 


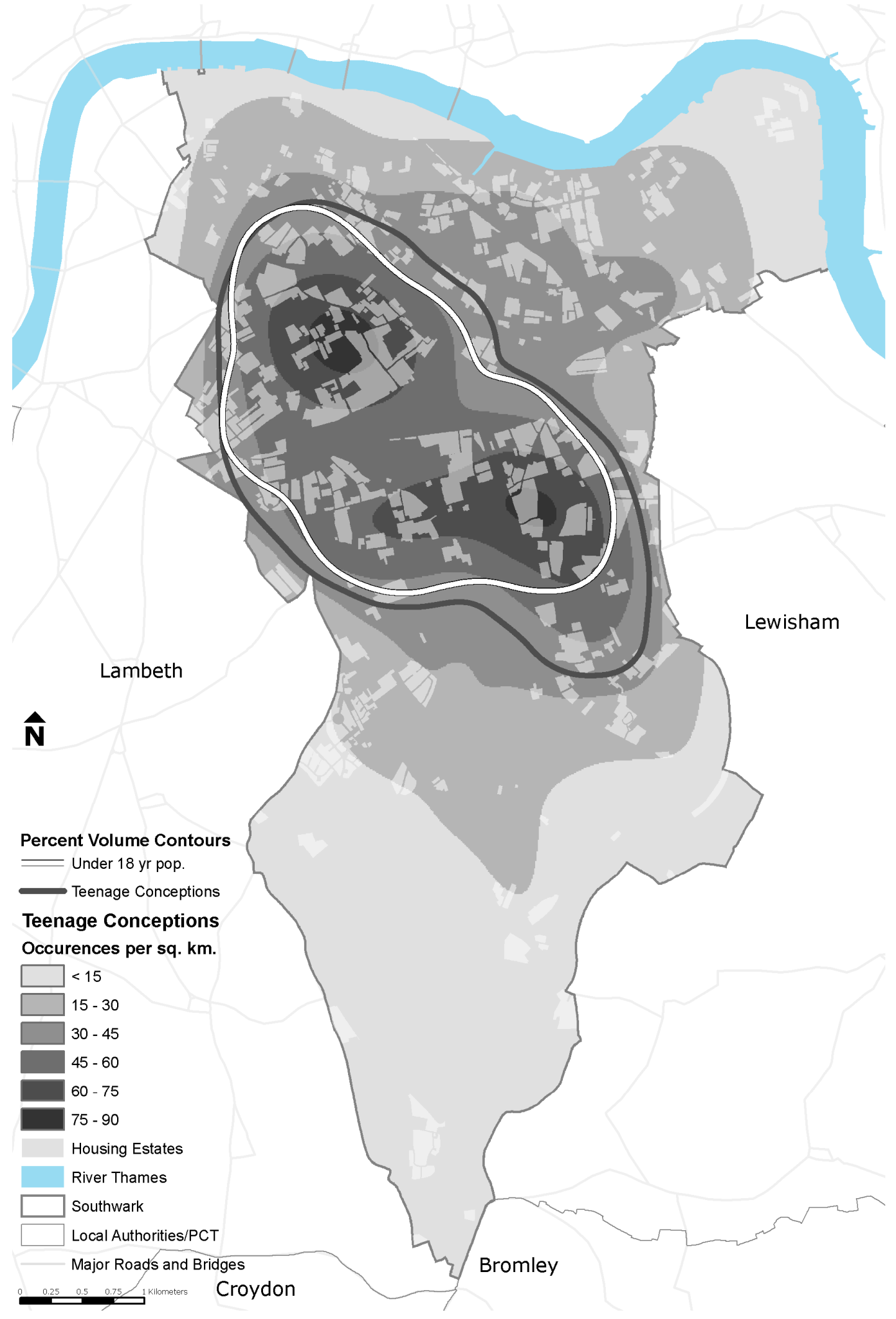

Figure 10 Percent volume contours of teenage conceptions versus under-18 year population. Bandwidth for kernel density estimation were $1000 \mathrm{~m}$. The contours show the top $50 \%$ percent volume contour 


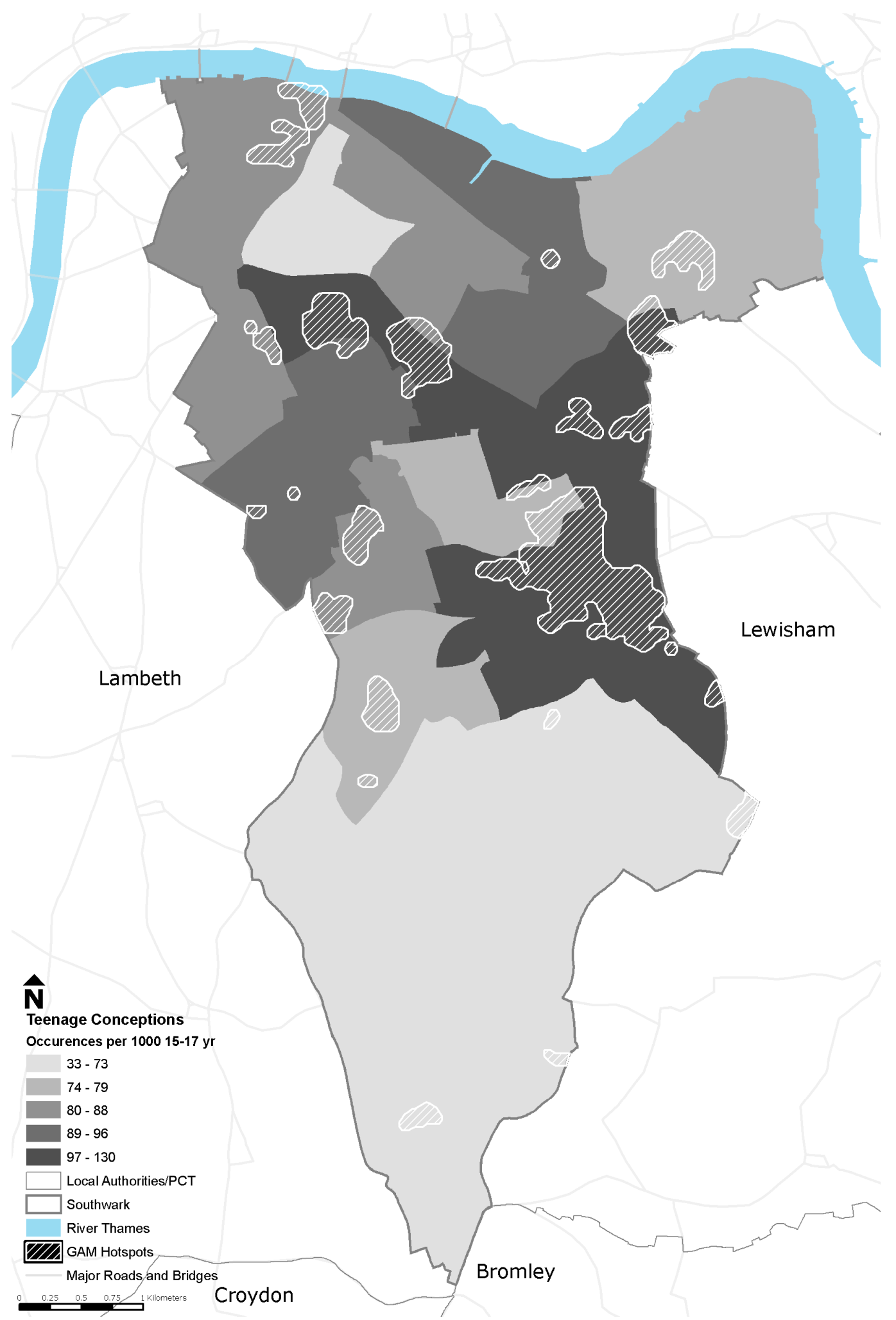

Figure 11 Teenage pregnancy hotspot map showing ONS-released rates (2001-03) and GAM hotspots for the local disaggregated data set. GAM search radius: $250 \mathrm{~m}$, cell size: $50 \mathrm{~m}$. 


\section{Teenage conception risk mapping}

A rich literature exists on the visualisation of spatially referenced incidence data, including methods to adjust local rates for variations in the underlying populations (Cressie, 1991; de Smith et al., 2008; Haining, 2003; Waller \& Gotway, 2004). One of the most basic methods of exploring spatial incidence data is 2D kernel density estimation (Atkinson \& Unwin, 2002; Silverman, 1986). This produces an unadjusted risk mapping that makes no assumption about the underlying population. It does, however, tell us something about the geographical concentration of teenage conceptions that in this case coincides with the locations of the biggest social housing estates in Southwark (Figure 10: an estimated 58\% of under-18 year olds in Southwark reside in social housing). To retain the information about the precise geographical location of the conceptions, while at the same time maintaining confidentiality in the visual display, percent volume contours (PVC) have been used. A PVC is the isoline which bounds a given percentage of the phenomenon in question. In this case the $50 \%$ isoline has been highlighted on the conception density surface. Comparing this to the $50 \%$ isoline of the under- 18 year population reveals a generalised indication of where conceptions are under- or over-represented. Figure 10 shows that an area to the south east of the main clusters appears to have more conceptions than would be expected from the underlying under-18 year old population figures. 


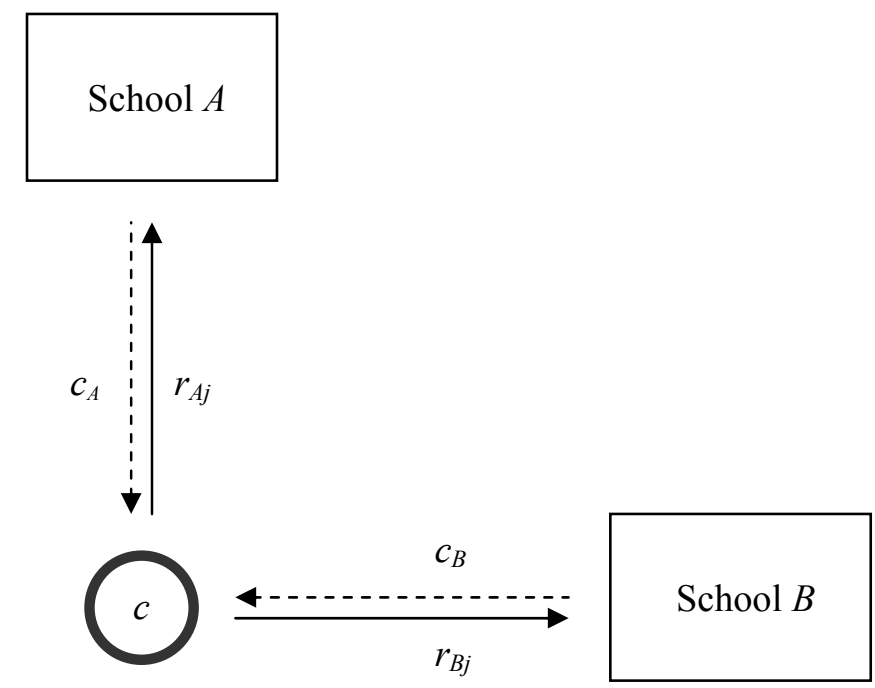

Figure 12 Recruitment flows and service point estimation (see text for explanation).

In order to evaluate the apparent concentration of conceptions, a cluster detecting algorithm, the Geographical Analysis Machine or GAM was applied (Gomez-Rubio et al., 2005; Openshaw et al., 1987). Using this method, both the numbers of cases and denominators are used to identify local rates within a specified search radius of the nodes of a fine grid overlain onto a base map. The resulting clusters are thus adjusted for the variation in the underlying population of under- 18 year olds. In this case the results confirmed the main ward level clusters evident from the official Office for National Statistics (ONS) data, but furthermore highlighted several smaller clusters that would have been overlooked by the ONS mapping (see Figure 11). This shortcoming arises because of the scale effects of aggregating data to population units as large as Census wards, and provides an instance of what is also known as the modifiable area unit problem (MAUP) (Openshaw, 1991). One much vaunted claim of the private sector geodemographics industry (Sleight, 2004) is that recourse to finer levels of granularity such as unit postcodes can reveal heterogeneity that is concealed at ward scales of analysis. The findings presented here concur 
with this general view but not necessarily that private sector geodemographic systems present the best solutions to public sector problems such as health profiling (Longley, 2005; Petersen et al., 2008)

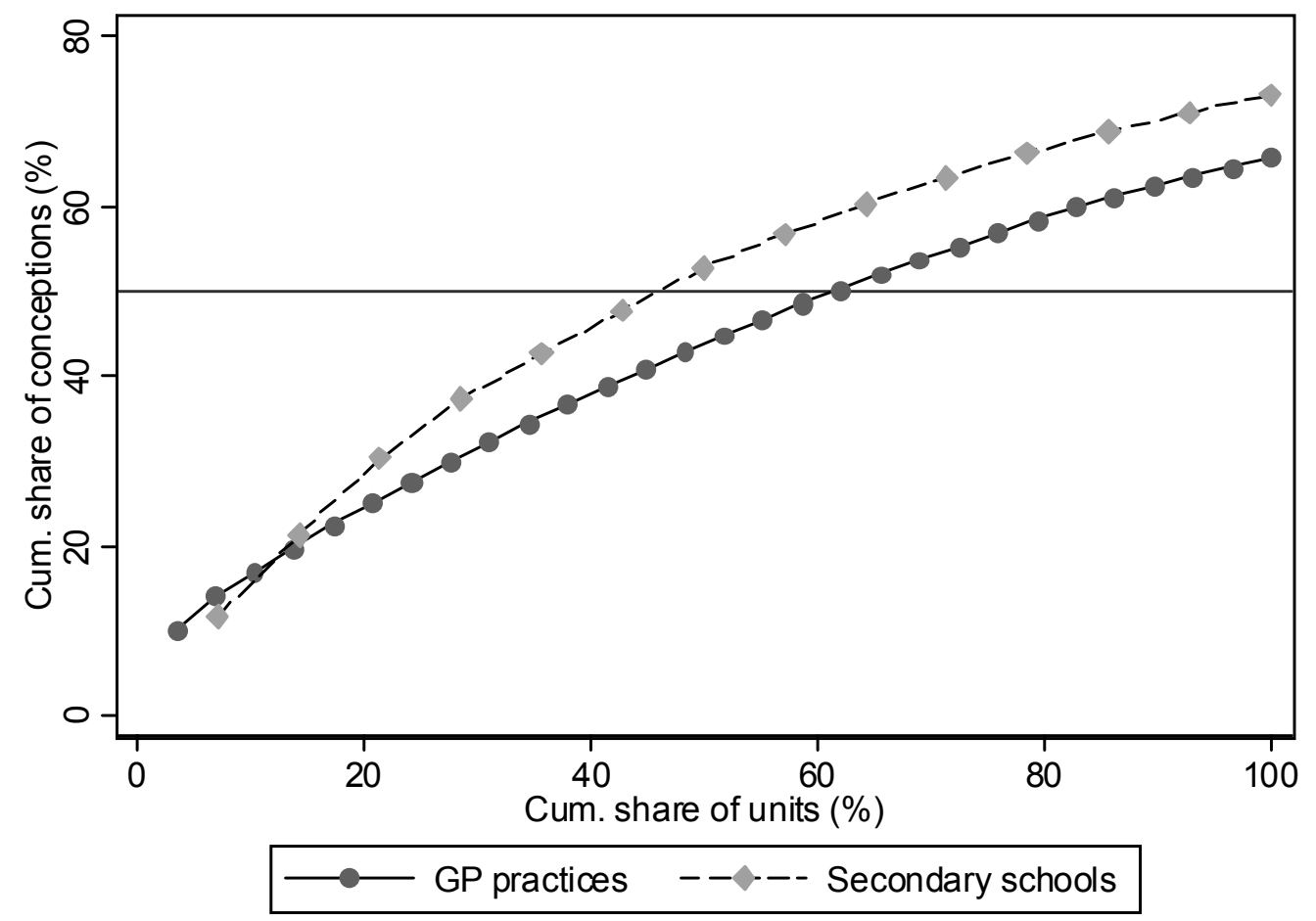

Figure 13 Gain diagram to reach target group by GP practices or secondary schools.

Kernel density estimation, PVCs and GAM are all useful exploratory tools for summarising high resolution spatial data without compromising data confidentiality of human subjects. They both can help to identify general or coarse patterning, and GAM can be used to highlight more localised clusters that might be missed in maps constrained to official administrative areas, whilst maintaining confidentiality. 
Table 11 Teenage conceptions estimated for state secondary schools. Schools anonymised: SS\#. Top $50 \%$ of conceptions could be reached by targeting minimum 7 secondary schools (Accumulated percentage: $\mathbf{5 3} \%$ )

\begin{tabular}{|c|c|c|c|c|c|c|c|}
\hline School & $\begin{array}{l}\text { Girls-at- } \\
\text { risk (n) }\end{array}$ & $\begin{array}{c}\text { Percentage } \\
\text { of all girls- } \\
\text { at-risk } \\
\text { among SS } \\
(\%)\end{array}$ & $\begin{array}{c}\text { Estmated } \\
\text { conceptions } \\
(\mathrm{n})\end{array}$ & $\begin{array}{c}\text { Percentage } \\
\text { of est. } \\
\text { conceptions } \\
\text { among SS } \\
(\%)\end{array}$ & $\begin{array}{c}\text { Relative risk } \\
\text { index among } \\
\text { SS }\end{array}$ & $\begin{array}{c}\text { Estimated } \\
\text { conceptions } \\
\text { all areas (\%) }\end{array}$ & $\begin{array}{c}\text { Estimated } \\
\text { conceptions } \\
\text { all areas } \\
(\text { Acc. \%) }\end{array}$ \\
\hline SS1 & 769 & 16 & 103 & 16 & 101 & 12 & 12 \\
\hline SS2 & 580 & 12 & 85 & 13 & 111 & 10 & 21 \\
\hline SS3 & 643 & 13 & 80 & 12 & 93 & 9 & 30 \\
\hline SS4 & 360 & 7 & 62 & 10 & 129 & 7 & 37 \\
\hline SS5 & 317 & 7 & 48 & 7 & 113 & 5 & 43 \\
\hline SS6 & 487 & 10 & 45 & 7 & 70 & 5 & 48 \\
\hline SS7 & 358 & 7 & 44 & 7 & 93 & 5 & 53 \\
\hline SS8 & 184 & 4 & 35 & 5 & 144 & 4 & 57 \\
\hline SS9 & 281 & 6 & 32 & 5 & 84 & 4 & 60 \\
\hline SS10 & 187 & 4 & 27 & 4 & 109 & 3 & 63 \\
\hline SS11 & 160 & 3 & 26 & 4 & 120 & 3 & 66 \\
\hline $\mathrm{SS} 12$ & 181 & 4 & 22 & 3 & 91 & 2 & 69 \\
\hline SS13 & 135 & 3 & 20 & 3 & 111 & 2 & 71 \\
\hline SS14 & 225 & 5 & 19 & 3 & 63 & 2 & 73 \\
\hline No School & - & - & 238 & - & - & 27 & 100 \\
\hline Total & 4867 & 100 & 885 & 100 & 100 & 100 & - \\
\hline
\end{tabular}

Table 12 Teenage conceptions estimated for GP practices. GP practices anonymised: GP\#\#. Top $50 \%$ of conceptions could be reached by targeting minimum 18 GP practices (Accumulated percentage: $\mathbf{5 0} \%$ )

\begin{tabular}{|c|c|c|c|c|c|c|c|}
\hline $\begin{array}{l}\text { GP practice } \\
\text { code }\end{array}$ & $\begin{array}{l}\text { Girls-at- } \\
\text { risk. Local } \\
\text { residents } \\
(\mathrm{n})\end{array}$ & $\begin{array}{c}\text { Percentage } \\
\text { of all girls- } \\
\text { at-risk } \\
\text { among GP } \\
(\%)\end{array}$ & $\begin{array}{l}\text { Estimated } \\
\text { conceptions } \\
(\mathrm{n})\end{array}$ & $\begin{array}{c}\text { Percentage } \\
\text { of } \\
\text { conceptions } \\
\text { est. among } \\
\text { GP }(\%)\end{array}$ & $\begin{array}{c}\text { Relative } \\
\text { risk index } \\
\text { among GP }\end{array}$ & $\begin{array}{l}\text { Estimated } \\
\text { conceptions } \\
\text { of total }(\%)\end{array}$ & $\begin{array}{c}\text { Estimated } \\
\text { conceptions } \\
\text { (Acc. \%) }\end{array}$ \\
\hline GP1 & 304 & 13 & 89 & 12 & 92 & 10 & 10 \\
\hline GP2 & 84 & 4 & 35 & 5 & 130 & 4 & 14 \\
\hline GP3 & 102 & 4 & 26 & 3 & 78 & 3 & 17 \\
\hline GP4 & 114 & 5 & 25 & 3 & 69 & 3 & 20 \\
\hline GP5 & 88 & 4 & 24 & 3 & 86 & 3 & 23 \\
\hline GP6 & 84 & 4 & 23 & 3 & 86 & 3 & 25 \\
\hline GP7 & 55 & 2 & 22 & 3 & 122 & 2 & 28 \\
\hline GP8 & 54 & 2 & 21 & 3 & 121 & 2 & 30 \\
\hline GP9 & 69 & 3 & 20 & 3 & 91 & 2 & 32 \\
\hline GP10 & 80 & 3 & 20 & 3 & 78 & 2 & 35 \\
\hline GP11 & 51 & 2 & 20 & 3 & 121 & 2 & 37 \\
\hline GP12 & 71 & 3 & 19 & 3 & 84 & 2 & 39 \\
\hline GP13 & 33 & 1 & 18 & 2 & 168 & 2 & 41 \\
\hline GP14 & 37 & 2 & 17 & 2 & 145 & 2 & 43 \\
\hline GP15 & 57 & 2 & 17 & 2 & 93 & 2 & 45 \\
\hline GP16 & 46 & 2 & 17 & 2 & 115 & 2 & 47 \\
\hline GP17 & 35 & 1 & 17 & 2 & 148 & 2 & 49 \\
\hline
\end{tabular}




\begin{tabular}{|c|c|c|c|c|c|c|c|}
\hline GP18 & 50 & 2 & 15 & 2 & 97 & 2 & 50 \\
\hline GP19 & 37 & 2 & 15 & 2 & 130 & 2 & 52 \\
\hline GP20 & 48 & 2 & 15 & 2 & 96 & 2 & 54 \\
\hline GP21 & 38 & 2 & 14 & 2 & 118 & 2 & 55 \\
\hline GP22 & 35 & 1 & 14 & 2 & 124 & 2 & 57 \\
\hline GP23 & 48 & 2 & 14 & 2 & 88 & 2 & 58 \\
\hline GP24 & 52 & 2 & 13 & 2 & 77 & 1 & 60 \\
\hline GP25 & 41 & 2 & 11 & 1 & 84 & 1 & 61 \\
\hline GP26 & 24 & 1 & 11 & 1 & 141 & 1 & 62 \\
\hline GP27 & 36 & 2 & 11 & 1 & 91 & 1 & 64 \\
\hline GP28 & 35 & 1 & 10 & 1 & 93 & 1 & 65 \\
\hline GP29 & 41 & 2 & 10 & 1 & 79 & 1 & 66 \\
\hline Other GPs & 492 & 21 & 167 & 22 & 106 & 19 & 85 \\
\hline No GP & - & - & 134 & - & - & 15 & 100 \\
\hline Total & 2341 & 100 & 885 & 100 & - & 100 & - \\
\hline
\end{tabular}

\section{Risk estimates for schools and GP practices}

Clusters based on residence alone may not necessarily be the most effective for targeted messages or strategies aiming to support young people. A different strategy is to use the most appropriate organisations in order to target individuals. Secondary schools and GP practices are often used in teenage pregnancy prevention campaigns and activities. This raises a resource allocation/optimisation problem: which schools and GP practices should be targeted in an evidence-based local strategy? The problem has been approached by 'allocating' each conception to schools and GP practices, respectively, based upon the underlying recruitment flow between residential postcodes in Southwark and secondary schools and GP practices. By recruitment flows are meant actual at-risk counts per postcode of individuals attending specific local schools and GP practices, calculated as:

$$
c_{i}=\sum c_{j} \frac{r_{i j}}{\sum r_{. j}}
$$

where $c$ is conceptions and $r$ is recruits (e.g. girls attending a particular school), $i$ is the service unit in question (e.g. a school or a GP practice), and $j$ is the residential unit postcode (Figure 3). If for example three conceptions, $c$, occurred in a given postcode, $j$, and 10 girls of secondary school 
age (11-17 year olds) are recruited to school $\mathrm{A}, r_{A j}$, and 20 girls to school $\mathrm{B}, r_{B j}$, then one conception is attributed to school A and two to school B, and so forth for all conceptions across all postcodes. The relative risk, $R R$, was estimated in the following way:

$$
R R_{i}=\frac{c_{i} / \sum c}{r_{i} / \sum r} 100
$$

In this way it was possible to rank and estimate relative risk for all secondary schools and GP practices (see Table 11 and Table 12). For public health purposes, the numerators (i.e. conceptions) may be just as important as relative risk measures (i.e. conceptions per girl-at-risk). In order to address at least $50 \%$ of all relevant conceptions, the results show that a campaign would need to include the 7 schools or 18 GP practices with the highest conceptions figures (Figure 13).

\subsection{Discussion}

\section{A local teenage pregnancy campaign}

National and local strategies to reduce teenage pregnancies follow a multi-agency and multimodal design incorporating school-based sexual health programmes, community-based education, contraceptive services, youth development and family outreach programmes (Social Exclusion Unit, 1999). This approach has been endorsed by recent reviews (Bennett \& Assefi, 2005; Swann et al., 2003). However, the lack of positive policy outcomes in inner city areas like Southwark suggests a need for a renewed and more focused approach. Where should a local evidence-based strategy focus? Which settings are the most effective for delivering messages about sexual health to young people? How are these settings identified? The mapping of risk across the Southwark PCT area has highlighted how much teenage conceptions vary locally. This in itself emphasises the need for a targeted approach. Hotspot maps (Figure 10, Figure 11) have the advantage of being intuitive and easy for public health staff to use in the planning of campaigns, as for example with the routing of a campaign bus with sexual health information. Another advantage of these 
maps is that they generalise locational information in a way that protects the confidentiality of any individual in the dataset.

Selecting hotspot areas is not, however, necessarily the most effective way of focusing a local strategy. As pointed out by some authors, hotspot mapping of apparent sexual health is a sensitive issue, and may have the negative outcome of adding to stigmatisation of neighbourhoods rather than achieving the intended end of empowering of young people and their families (Arai, 2007). This is clearly one of the dilemmas that face public health departments: to intervene without undermining the trust of participants or aggravating their circumstances in an indirect way by identifying their neighbourhood as a 'bad' place to live. Arai (ibid.) also advocate a 'normality by locality' view for a more general acceptance of teenage motherhood.

The counter-arguments are that leaving teenage pregnancy as a private matter is a way of respecting the autonomy of individuals rather than creating autonomy. Viewed from this perspective, anti-teenage pregnancy campaigns can be thought of not a response from a perfectionist and paternalistic State to restrict personal freedom, but rather as a way of putting personal freedom in a longer perspective that involves greater choice of education, family and lifestyle (Holland, 2007). In this context, for example, it is important to note that those undergoing pregnancy in their teens are, as a consequence, more likely to drop out of school and experience diminished life chances as a consequence (Barnet et al., 2004; Social Exclusion Unit, 1999; , 2006; Swann et al., 2003). My view is that PCTs should provide the type of services and support that young people would like combined with a greater understanding of peer group influences, and the findings of some research supports this agenda (Crosnoe \& McNeely, 2008; DiCenso et al., 2001; Pearson et al., 2006). Consultations of young people about their experiences with sexual health education, for example, have led to a change in the emphasis of interventions from anatomy and scare-tactics to the advocacy of negotiation skills in sexual relationships and better contraceptive services. There have also been calls for wider advertising of local services, 
for example in shopping centres and other public places (DiCenso et al., 2001). This does, however, suggest a role for hotspot mapping when locating many essential services.

Teenage pregnancy rates are amongst the new social targets that have been suggested for school performance auditing (Department of Children Schools and Families, 2007; Lipsett, 2008). Looking more broadly at health related issues (aggression, alcohol and drug use) in adolescence, then there are evidence to suggest that schools do play a role above the potentially confounding factors studied such as prior health status, parental and neighbourhood effects (West et al., 2004) and some would like schools to work specifically with improving their ethos (Bonell et al., 2007). Others point to peer influences - negative or positive - within the school classrooms and not just school units per se (Johansen et al., 2006). There also seems to be a lot yet to be gained from studying social networks in relation to teenage pregnancy and effectiveness of interventions using such insight (Crosnoe \& McNeely, 2008; Pearson et al., 2006).

In this case study, the need for directed action was important for the local strategy implementation. Both secondary schools and GP practices were considered as appropriate agents. Selecting the organisations most likely to be in the closest contact with the target group in many ways moves beyond the limits of hotspot mapping; one could say from event to action. Dealing with these organisations compared to hotspots had several advantages. They represent wellestablished networks of institutions with professional staff in daily contact with young people. This makes interventions easier and more cost-effective to deliver. In this case the estimation of conceptions for each school helped significantly to engage local schools in new sexual health programmes. By contrast, the official Local Authority and ward level figures made it much harder to provide the evidence about conception rates and thus also more difficult to engage school partners. 
Although being the most appropriate organisations for local policy implementation there are also indications that this is an area that needs improvement not only in the targeting, but also in the substance of the programmes. School-based programmes promoting contraception resulted in higher uptake of contraception relative to abstinence-only programmes (Bennett \& Assefi, 2005). However, a systematic review of school-based teenage pregnancy prevention programmes reported no overall success in reduction of pregnancies (DiCenso et al., 2002).

Compared to GP figures there were a higher proportion of cases in which it had to be assumed that the individuals were not registered with a local school (27\% versus $15 \%$ : see Table 11 and Table 12). This could be interpreted as an association between school dropout rates and teenage pregnancy occurrences either as an effect (suggested in cohort study by Barnet et al., 2004) or a cause. It must also be stressed that, although these are interesting hypotheses, they are beyond the limit of the study design used here.

The purpose of the method described here is to provide an easy and cheap method to predict the most likely schools and GP practices from routinely collected data for targeted interventions. The coupling of the data sets by postcode rather than by any individual identifiers (such as name, address, date of birth, NHS number, etc.) should have fewer implications for data protection and individual privacy. In the current practice postcode is considered identifiable information and as such subject to ethical approval from government bodies (Department of Health, 2003). In a recent survey concerning the collection, storage and analysis of cancer registry data however only $8 \%$ of responders objected to the use of full postcode, while the proportion of objectors rose to $16 \%$ on a question of whether to store personal name and full address (Barrett et al., 2006). The debate about patient consent and rights with respect to the accumulation of identifiable data by health authorities continues (e.g. McGilchrist et al., 2007) as do calls for greater integration of medical databases for epidemiological gains (e.g. Mladovsky et al., 2008). With the method 
described here however the intention has been to demonstrate that data sets collected for local service purposes do not need to be fully 'identifiable' but can be effective even if it only contains small area counts with no other identifiers.

There is at present little evidence to suggest that GP practices provide useful foci for teenage pregnancy intervention. It has however been argued that some young people may prefer the relative anonymity of attending a general as opposed to a specialist sexual health clinic (Tripp \& Viner, 2005). More resources are now directed towards primary and community healthcare and GP practices are rewarded for matching services to local needs in their new contracts with the Department of Health (Department of Health, 2006). Estimating conceptions for each GP is another way in which a trust can continue to make this issue 'visible' and relevant for local stakeholders.

Finally, consideration of teenage pregnancy and other adolescence health issues are complicated by peer group influences (Crosnoe \& McNeely, 2008) that may lie beyond the control of families, family doctors and school head teachers. Target setting (as in performance indicators) for teenage pregnancy rates may for this reason not be well-received. Targeted strategies based on local evidence, as suggested in this paper, however can provide a new focus for the many local initiatives to reduce unwanted teenage pregnancy.

\section{Targeting of organisations in a social marketing context}

As discussed in the introduction to chapter 5, an important discipline within the geographical information science domain has been the definitions of new ontologies and semantics for the formalisation and representation of spatial data. The application of spatial analysis has also led to theoretical contributions in many disciplines including healthcare. Healthcare accessibility is one of these areas and although public health concerns a broader spectrum of functions, the work on 
accessibility has many similarities to the fundamentals of social marketing. Healthcare accessibility is especially concerned with barriers for uptake, whereas social marketing is concerned with barriers that prevent individuals from engaging with programmes (see section 3.2). Khan's access dimension model is particularly useful in this perspective, where healthcare access may be realised with the help of certain facilitators or hindered by barriers (Kahn, 1992) (Figure 13). Access may also be perceived as either geographical or social.

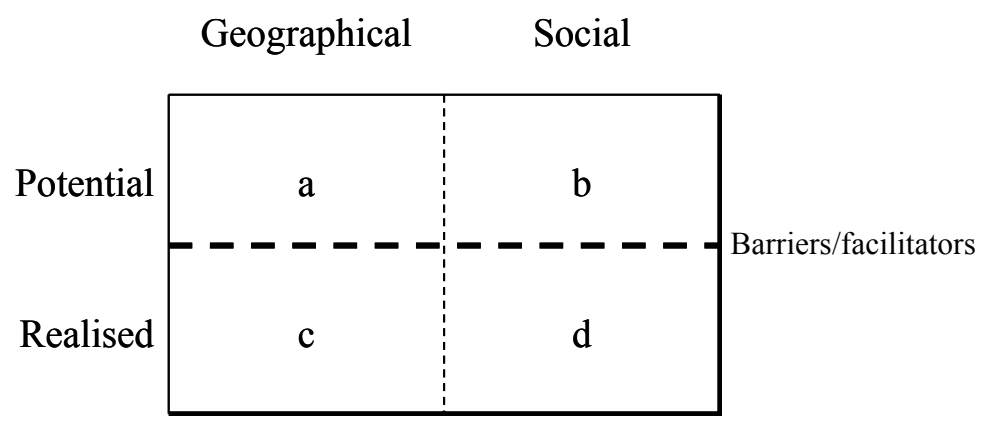

Figure 14 Dimensions of healthcare accessibility (adapted from Khan, 1992)

The main barrier to geographical access is distance; typically measured in Euclidean distance or as travel time. The importance of distance, which could be conceived as an environmental variable, fits well with the cartesian schema of geographical information systems. Many studies demonstrate the power of geographical analysis in healthcare accessibility studies especially in rural areas (e.g. Lovett et al., 2002; Schuurman et al., 2006). In cities the effects of distance are naturally less pronounced and so the existence of social barriers comes to the fore. Cummins et al. (2007) describes the 'traditional' view of healthcare accessibility as one with service points fixed in space and analysts obtaining futile results due to the wilful assumption that utility of services is decreasing with distance in the plane. A 'relational' view would in contrast recognise that services are 'layered' in their availability to 'populations via varying paths in time and space [and that] Euclidian distance may not be relevant to utility' (Cummins et al., 2007). While this is good point, it does not seem in conflict with Kahn's (1992) much earlier work with the two- 
dimensional access model (Figure 14). Social access may, for instance, have separate barriers, but at the same time be manifested geographically, if hard-to-reach groups are distributed in that way. An epidemiological approach to prevention of teenage pregnancy - as a health outcome would be to establish causes and trajectories and maybe to map out the most 'incriminating' risk factors. Teenage pregnancy is however a sensitive and personal sexual health issue with few local data to support this type of analysis or mapping. The interventions instead follow a multi-modal and multi-agency approach (38 organisations were involved in the Southwark campaign not counting schools, youth clubs or housing estates). Operational issues had to be taken into account too and so concentrating on organisations, rather than areas, were important. As a data model the work with teenage pregnancy is an example of a relational view on healthcare users. The methodology presented in the chapter was thus concerned with flows of pupils or patients in organisational networks between homes and services in a fine scale geographical data model. Schools are often chosen as foci for facilitating change in health behaviours, but the local campaigns had in the past experienced some fatigue and disbelieve by school management, when approached for their collaboration in 'another sexual health campaign'. Creating and providing timely and specific evidence therefore proved to be an important motivating factor - not for the agents (teenagers), but for the facilitators (school management teams, Figure 14). 


\section{Chapter 7 Targeting based on areal aggregations}

The problems and assumptions associated with the analysis of aggregated data is a wellknown problem across many disciplines (Cho \& Manski, 2008; Cummins et al., 2007; King et al., 2004; Manski, 1995; , 2003). Manski (1995) asserts that no amount of statistical 'sampling', data collection and empirical efforts can ever fully explain any real world phenomena except in the critical light of its assumptions. He calls this the identification problem of social science; a problem that supersedes statistical inference. From any given design we have to acknowledge the assumptions it is based upon and only from that can we determine which questions might be answered from such design and with what level of 'credibility'. To quote Manski: 'the credibility of inference decreases with the strength of the assumptions maintained' (Manski, 2003).

This chapter focus on the application of geodemographics as a method of targeting in public health campaigns. As a methodology it relies on the integration of data and information on health, health behaviours, lifestyles and neighbourhood characteristics. For the purpose of the further discourse this field can be termed: health geodemographics.

The main assertion in health geodemographics is that correlated but disparate data sets from its underlying domains may easily be coupled to form predictions for health promotion and evidence-based policy. It is, as described in the introduction to chapter 5, a knowledge creating process that depends on a leap from the informal to the formalised (formalisation). The leap is arguably greater in this context, because it involves not just one classification but the coupling of several. The ends of health geodemographics may well justify its means, but without critical examination of assumptions, criteria and limitations it would equally be a naïve and fallible one. 
7.1 Health, lifestyles and neighbourhood - a triple construct?

Health geodemographics promises an easy and cheap way to identify geographical neighbourhoods for health promotion campaigns based on estimates of health behaviours imputed from the three main variables: health, lifestyles and neighbourhoods. All three variables are meanwhile unobservable in themselves and we need to rely on a selection of indicators in order to gain any knowledge about them. They are in other words theoretical constructs 'synonymous with concepts ...abstractions aimed at organizing and making sense of our environment. [The validation of constructs are] fraught with difficulties, ambiguities, even circularities' (Pedhazur \& Pedhazur Schmelkin, 1991, p.52). If we consider health in itself, we have already established in part one that it is possibly a double construct of disease-absence and wellbeing. The former can be measured negatively through disease diagnoses for every known disease, the latter through indicators of material or social needs including indicators of resources afforded to the individual and other determinants of individual capabilities. The question is how well indicators achieve this, i.e. how the construct can be validated. This process is in other words 'concerned with validity of inferences about unobserved variables (the constructs) on the basis of observed variables (their presumed indicators)' (Pedhazur \& Pedhazur Schmelkin, 1991). The process can broken up into two parts, internal validity and external validity. Threats to internal validity are usually summarised as bias and this chapter will begin with an examination of known biases to internal validity from the epidemiological, sociological and social health literature. This is not to say that health is 'unmeasurable', but because the bias question purposefully forms a structure for a discussion of what can and cannot reasonably be known from studying georeferenced databases of routinely collected healthcare data. External validity in turn concerns the extent to which findings can be generalised to other settings outside those where the insight was gained. 


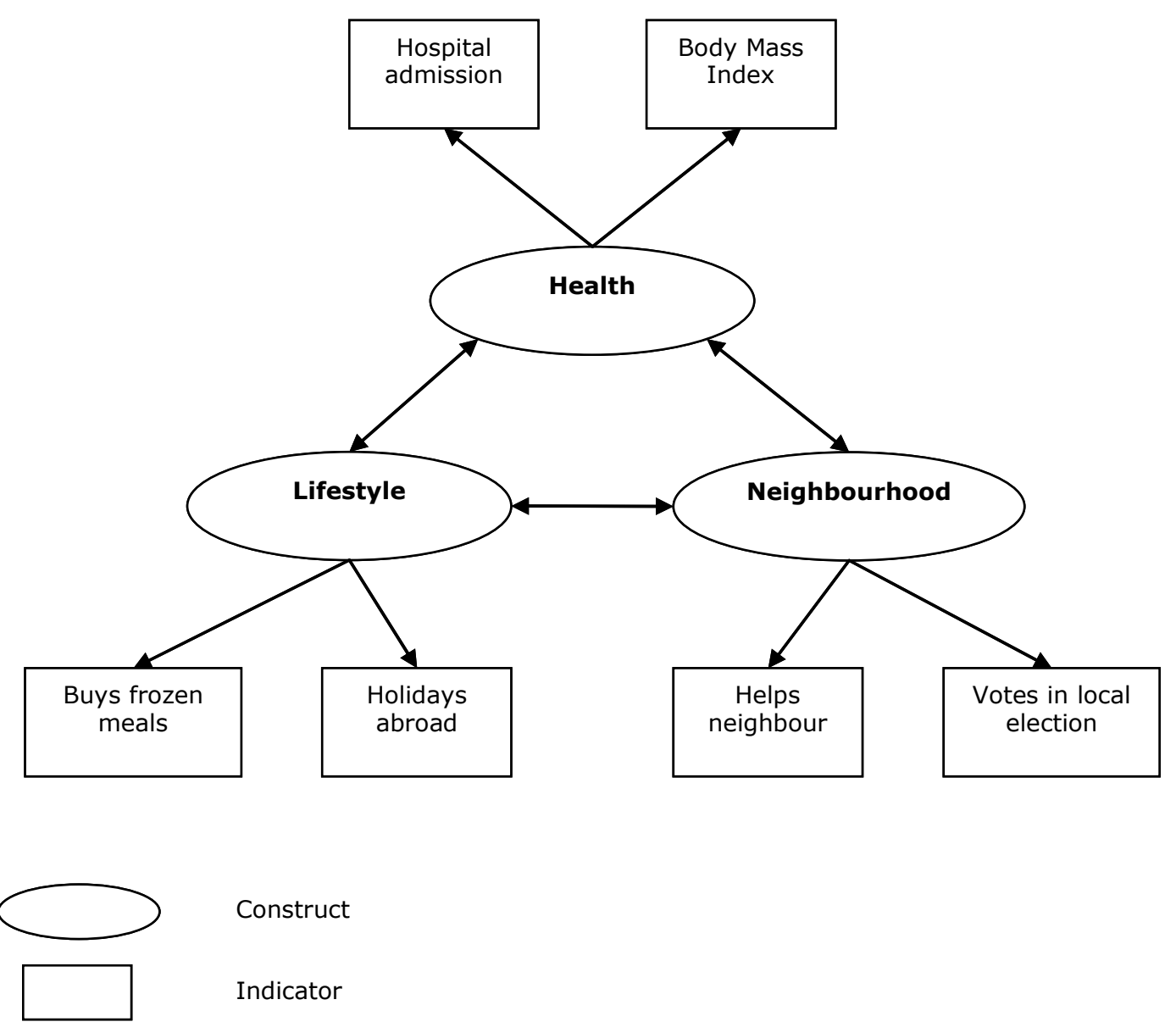

Figure 15 Health-lifestyle-neighbourhood super-construct

If we agree that health can be measured with some uncertainty by a number of indicators, we then need to extend this idea to include lifestyles and neighbourhood type. Does this give us a 'super-construct' with at least three unobservable variables? (Figure 15). Some suggest structural equation models or instrumental variable regression as a way of validating potential causal pathways between a host of observable and unobservable variables. The course taken here however is to explore methods to gain insight from routinely collected data bearing in mind that causation in an area as complex as health requires carefully designed epidemiological studies, where the many intervening and correlating factors can be dealt with more convincingly. The real power of observational database studies however is their ability to detect change in real-time and 
to propose new working hypotheses for the dynamic interactions between health, lifestyle, neighbourhood type and individual agency.

Health geographers face two separate issues with regards to areal aggregations. The first type of problems are often categorised as ecological inference. The second type concern the analysis of the hierarchical structure of otherwise disaggregated data, which in the geographic literature is often referred to as neighbourhood-effects (or effects of 'place' or area). The hierarchy may also be perceived as organisational and not only geographical, e.g. the effects that might be discerned from analysing data at a group level (e.g. hospital units) in addition to individual and compositional factors. The following concentrate on bias in relation to observational studies within a geographical framework as relevant to the sections on geodemographics and studies of healthcare databases.

\subsection{Ecological inference}

Ecological inference problems arise when the researcher only has data available at an aggregate level. In political science a typical instance may be where the researcher would like to calculate the support a political candidate has from Black versus White voters ${ }^{8}$. In the US the percentage of Black and White adults in a precinct is known from the Census. Assuming that everyone from a precinct turns out on election day, the Black versus White proportion of votes for the different candidates are then simply assigned proportionally from the 'racial' composition of that precinct. This can easily be demonstrated in a $2 \times 2$ contingency table for a two candidatestwo 'races' situation. However, in many cases numbers are sufficiently small or skewed to make further assumptions, e.g. what the likely confidence intervals should be to a point estimate. If for example a precinct has 290 Whites and 10 Blacks it is possible to work with a closure type

\footnotetext{
${ }^{8}$ See chapter 5 for a more detailed comment on the race versus ethnicity terminology
} 
assumption, that if all 10 Blacks voted Republicans (e), then 10 Whites must have voted Republican too (b) if the Republican candidate had 20 votes in all, which leaves the Democrats with $100 \%$ White votes, etc (Figure 16). The other extreme would be that no Blacks voted Republican $(e=0, b=20, a=270, d=10)$ and so the Democrats proportion of White votes could range from $270-280$ out of possible 300 votes.

Figure 16 Ecological inference problem in a $2 \times 2$ contingency table

\begin{tabular}{|c|c|c|c|}
\hline & Democrats & Republican & \\
\hline White & $\begin{array}{c}\mathrm{a} \\
(280 ; 270)\end{array}$ & $\begin{array}{c}\mathrm{b} \\
(10 ; 20)\end{array}$ & $c=290$ \\
\hline \multirow[t]{2}{*}{ Black } & $\begin{array}{c}\mathrm{d} \\
(0 ; 10)\end{array}$ & $\begin{array}{c}\mathrm{e} \\
(10 ; 0)\end{array}$ & $\mathrm{f}=10$ \\
\hline & $\mathrm{g}=280$ & $\mathrm{H}=20$ & $i=300$ \\
\hline
\end{tabular}

More complicated assumptions can be brought into play, e.g. whether Black residents are less likely to register to vote or not and if so, whether there are any spatial or temporal nonstationarity to this behaviour (Fotheringham, 2000). King et al. (2004) have reviewed and proposed generalised linear models for estimating ecological inference problems. From the perspective of problem identification, however, there is a fundamental indeterminacy to the ecological problem. It represents an inverse problem with a multitude of possible 'solutions'. Some classify it as a partially identified problem due to the fact that it is after all possible to estimate confidence intervals or ranges in many cases (Cho \& Manski, 2008).

\subsection{Bias}

Bias is 'any systematic error in the design, conduct or analysis of a study that results in a mistaken estimate of an exposure's effect on the risk of disease' (Gordis, 2004). The types of bias discussed here are primarily those that can be ascribed to lack of internal validity for a study. There are other biases pertaining to data interpretation, literature selection and citation which open up questions of external validation that lie beyond the scope set out for this section (see e.g. 
Choi, 2000). Delgado-Rodriguez and Llorca (2004) have extensively reviewed bias in the epidemiological literature. The aim with the following is to amend and discuss a subset of the biases listed in their paper with emphasis on observational studies as relevant to the subsequent analyses of geodemographics and health outcomes (Table 13). The discussion will also draw on recent reviews from the sociological and medical geography literature to discuss spatial effects and bias relevant in this area (Cummins et al., 2007; Entwistle et al., 2007; Riva et al., 2007). Delgado-Rodriguez and Llorca (2004) identify three main groups of bias; 1) selection bias, 2) information bias, 3) confounding and interactions in disease causation.

Table 13 Bias types in disease causation. Modified from Delgado-Rodriquez and Llorca (2004) with emphasis on the observational study design. Amendments indicated with *

\begin{tabular}{|c|c|c|c|}
\hline Bias Group & Bias Subgroup & Specific bias & Study design affected \\
\hline \multirow[t]{13}{*}{ Selection bias } & Ascertainment bias & Competing risks & All studies \\
\hline & & Length biased sampling & $\begin{array}{l}\text { Cross sectional study, } \\
\text { screening }\end{array}$ \\
\hline & & $\begin{array}{l}\text { Survivor treatment selection } \\
\text { bias }\end{array}$ & Observational study \\
\hline & & Settler-wanderer bias* & Observational study \\
\hline & & Unhealthy survivor* & Observational study \\
\hline & & $\begin{array}{l}\text { Prevalence-incidence bias. } \\
\text { a) Lifestyle change* or Sick } \\
\text { quitter bias; } \\
\text { b) Selective survival bias }\end{array}$ & $\begin{array}{l}\text { Cross sectional study, } \\
\text { case-control study with } \\
\text { prevalent cases }\end{array}$ \\
\hline & & Cash and Care bias* & Observational study \\
\hline & Healthcare access bias & Centripetal bias & Observational study \\
\hline & & $\begin{array}{l}\text { Diagnostic/treatment access } \\
\text { bias }\end{array}$ & Observational study \\
\hline & & Popularity bias & Observational study \\
\hline & & Referral filter bias & Observational study \\
\hline & $\begin{array}{l}\text { During study } \\
\text { implementation }\end{array}$ & $\begin{array}{l}\text { Missing information in } \\
\text { multivariable analysis }\end{array}$ & $\begin{array}{l}\text { All studies (mainly } \\
\text { retrospective) }\end{array}$ \\
\hline & & Non-response bias & Observational study \\
\hline
\end{tabular}




\begin{tabular}{|c|c|c|c|}
\hline & Social selection & Health selection* & Observational study \\
\hline & Self selection & Choosing neighbourhoods * & Observational study \\
\hline & & Social ties* & Observational study \\
\hline & & Moving to healthcare* & Observational study \\
\hline & & $\begin{array}{l}\text { Healthy migrant/unhealthy } \\
\text { re-migration effect } *\end{array}$ & Observational study \\
\hline & & Retirement emigration* & Observational study \\
\hline \multirow[t]{14}{*}{ Information bias } & Misclassification bias & $\begin{array}{l}\text { Differential misclassification } \\
\text { bias }\end{array}$ & All studies \\
\hline & & $\begin{array}{l}\text { Non-differential } \\
\text { misclassification bias }\end{array}$ & All studies \\
\hline & & Observer/interviewer bias & All studies \\
\hline & & Detection bias & Screening study \\
\hline & & Lead-time bias & Screening study \\
\hline & & Recall bias & All studies \\
\hline & & Measurement bias & All studies \\
\hline & & $\begin{array}{l}\text { Incomplete or inaccurate } \\
\text { data recording* }\end{array}$ & All studies \\
\hline & Reporting bias & Family aggregation bias & Observational study \\
\hline & & $\begin{array}{l}\text { Mode for mean bias (central } \\
\text { tendency bias) }\end{array}$ & All studies \\
\hline & & Obsequiousness bias & All studies \\
\hline & & $\begin{array}{l}\text { Unacceptable } \\
\text { disease/exposure bias }\end{array}$ & All studies \\
\hline & & Underreporting bias & All studies \\
\hline & Ecological fallacy & Ecological bias, MAUP & Ecological study \\
\hline Confounding & & Confounding & All studies \\
\hline Interaction & & Effect modification* & All studies \\
\hline
\end{tabular}




\section{Selection bias}

Selection biases concerns the selection of study objects and the biases that can arise for the exposure-disease association when the attributes of the selected subjects are crucially different to the rest of the population (Table 13).

The first kind of selection bias is known as ascertainment bias. This is the type of problem, where the risk factor (or exposure) is associated with the detection of the disease (or health outcome) rather than with a root cause.

Healthcare episodes are usually classified with a clinical diagnostic code, however a particular case may warrant more than one diagnosis; a patient may be e.g. diagnosed with both diabetes mellitus and congestive heart failure simultaneously. Only one of those will be entered as the primary diagnosis. This is the competing risks bias. A study using primary diagnoses as sampling frame may thus lose information about more complicated cases due to competing risks.

Health outcomes are often reported either as incidence, new cases per population per unit of time, or prevalence, accumulated sum of cases per population per unit of time. The decision to report one or the other typically depends on the duration of the outcome or how rare it is, e.g. influenza is usually reported as incidence, whilst asthma is reported as prevalence. Prevalenceincidence bias refers to instances of selection bias, where exposure-disease causation is inferred from prevalence rather than incidence in the population. The causal relationship or association may in such cases be biased by two things; a) if the diagnosed individual changes lifestyle subsequent to the diagnosis (we could term this lifestyle change bias although it comes close to the so-called sick quitter bias); or b) the diagnosed (and selected) individuals are the survivors of the condition in question and have attributes that are different to those that have already died; this instance of the prevalence-incidence bias is also known as selective survival bias. An extreme instance of the latter would be if the survivors of a condition $\mathrm{X}$ have attributes that are different to the reference population, say they eat more daily portions of vegetable than the average person, 
whilst those that also were diagnosed, but have already died, ate fewer. If the deceased fraction of the individuals constituting the prevalence is not included in the study, a conclusion might be that 'eating lots of vegetables cause condition X', which would effectively be a reversed causation. Selective survival is primarily a problem, when the risk factors influence mortality, whereas lifestyle-change bias (and the related sick quitter bias) would apply more generally. The effects of lifestyle changes are noticeably difficult to detect from the observational studies discussed here and would be detected more accurately in a longitudinal study design.

A related problem, length-biased sampling, arises when cases with a longer duration result in more recorded episodes and hence are also more likely to be included in surveys, despite the fact that this group may not be representative for the condition overall. Similarly, if some patients have a more complete record of both their outcome and exposure attributes they will for that reason alone contribute more to the inference about causes, but they may not otherwise be representative of the reference population, i.e. leading to the missing information in multivariable analysis bias. In areas with a high population turnover, patients that are resident for longer will be more likely to be registered with the healthcare services and so there is a risk that more information and inference will be based on that proportion of the population although they may not be representative of the population overall. We could term this the settler-wanderer bias (where the term 'wanderer' simply signifies anyone with temporary residence in an area; a nonsettler). The settler-wanderer bias is a more general case of what MacIntyre and Ellaway (2003) refer to as the unhealthy survivor bias, where those that remain in their childhood neighbourhood hypothetically are the less healthy and less educated, whilst the better educated and more dynamic have moved elsewhere. Survivor treatment selection bias is again related to this problem only where patients who live longer have greater chance of receiving treatment $\mathrm{X}$ yielding a positive but problematic association between that treatment and survival. A different side to this problem is the effects of population turnover, which with time amplifies the chronic healthcare needs in low turnover neighbourhoods (Figure 17). 
Figure 17 Population turnover and healthcare needs. Circles symbolises a neighbourhood at two points in time. Arrows symbolises migration equilibrium.

\begin{tabular}{|l|l|}
\hline Neighbourhood Turnover & \multicolumn{1}{|c|}{ Healthcare focus } \\
\hline Low turnover neighbourhoods \\
Demography: ageing \\
\hline Higration \\
Health status: increasing prevalence of chronic \\
conditions
\end{tabular}

Health seeking behaviour is a topic in its own right. The patients that seek the attention of services may in general be healthier than those that do not respond, i.e. leading to the nonresponse bias or healthy volunteer bias in which genuine health problems in the population are underestimated (see also reporting bias below). 
An even more profound problem is bias from social selection (also known as health selection) (Chandola et al., 2003). This is the question of whether patterns of ill health are caused by adverse social circumstances (i.e. social causation) or whether people with poor health may be limited in terms of improving their social position and tend to have lower paying and unskilled jobs. In neighbourhood terms they would as a result 'select' themselves into deprived neighbourhoods. The health selection hypothesis has been studied in a number of longitudinal studies and there do not seem to be any overwhelming evidence for it. This is frequently taken as attributive support for neighbourhood initiatives to address social deprivation and social mobility, e.g. by improving schools and other local services in the more deprived areas (Cummins et al., 2007; Davey Smith, 2003; Wilkinson, 2005).

The discourse on social selection versus social causation concerns causation in a life-course perspective. There are a number of similar biases and effects, which could equally be called 'social selection', but which here will be discussed as self selection: a 'lighter' version of social selection without the social determinism implied in the lifecourse paradigm. The first of these could be termed choosing neighbourhoods. This is the observed fact that choice of neighbourhood is not random, but often reflects a trade-off between different needs, wants and financial constraints including normative climate, socioeconomic and ethnic composition, safety, accessibility, geographically determined eligibility to a school and quality of the built environment (Entwistle, 2007). The choosing neighbourhoods bias 'upsets' the natural experiment whereby the observer would infer effects from causes. Take e.g. an ecological study that concludes that 'walking in natural surroundings is beneficial for your health'. Maybe people who live near places where they can go for these walks, however, had better health before they made their neighbourhood choice and so walks don't cause good health; walks and good health are simply correlated.

The resilience or quality of a neighbourhood can further depend on the social ties or networks (Figure 18). The mapping of both social networks and agency of individuals and community 
groups are interesting avenues for research into social and neighbourhood effects in health (Entwistle et al., 2007); although it should also be noted that such information is rarely accessible for secondary data analysis.

\section{Sibling Tles}
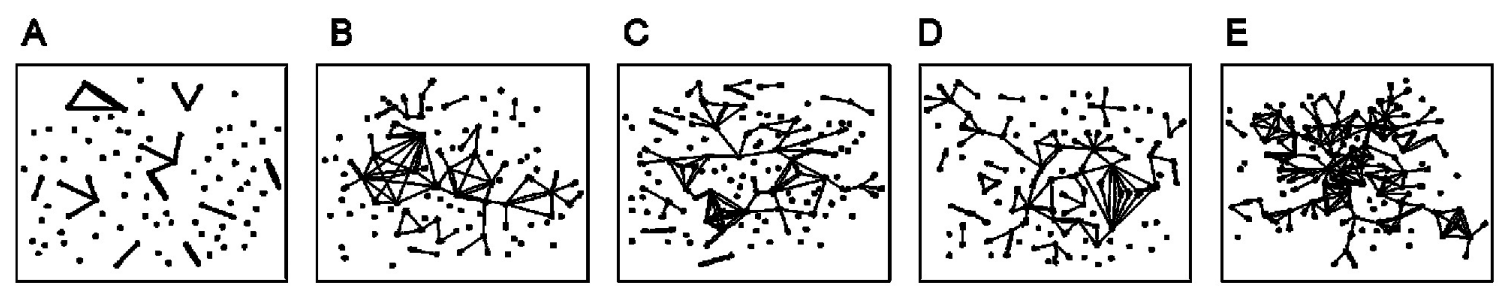

\section{Help with the Rice Harvest}
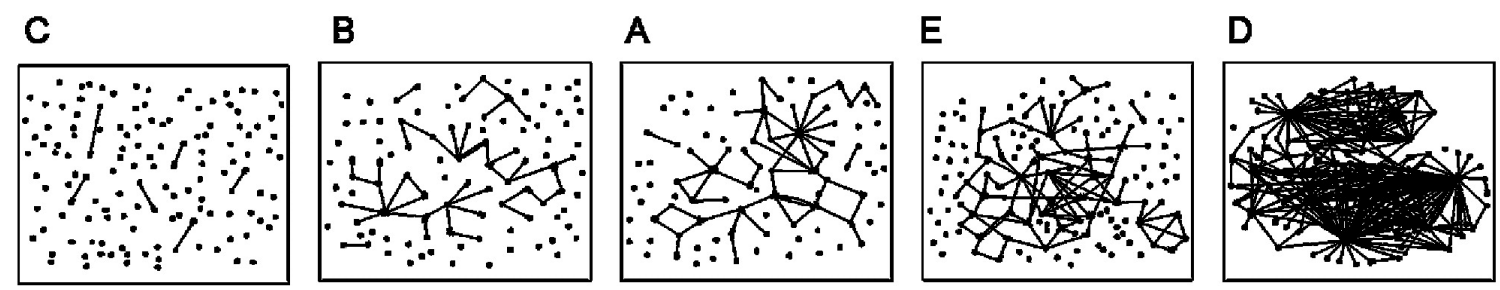

Figure 18 Social ties and neighbourhood resilience in five villages in Nang Rong, Thailand. NB order of villages differ in the two panels; ABCDE versus CBAED (From: Entwistle et al., 2007)

Other spatial effects regarding neighbourhoods and health status are observations that people with poor health may move closer to popular healthcare facilities, which can create reverse cautions between good accessibility and poor health (Entwistle, 2007); i.e. a moving-tohealthcare bias. In relation to international migration it is often observed that migrants have selectively better health than the average in both donor and host country, the healthy migrant effect. The reverse effect has also been proposed, the unhealthy re-migration effect, where migrants who fall ill hypothetically are more likely to return to their home country for treatment and retirement (Razum et al., 1998). In that context it also seems appropriate to mention retirement emigration; e.g. an estimated one million Britons were in retirement abroad in 2006 (Sriskandarajah \& Drew, 2006). 
Part of the NHS reforms is to make the organisation more market-oriented, to work on user satisfaction and to treat users as customers or 'choosers' in a quasi-open market with competition between healthcare providers (Pollock et al., 2007). The NHS now e.g. publish quarterly performance results for hospitals and users may choose to be treated in a hospital different to their nearest. Often choices may be constrained in some way, but potentially we may see a greater role for user preference in the system opening up for healthcare access bias, where the users of a particular service are different to the local population. In some cases, e.g. in hospital episode statistics (HES), the records will eventually be coded with the users' codes of residence as well as the site codes for the hospital. Nevertheless there are potential biases in analysing data that have not been 'normalised' in such a way or from the fact that use of private hospitals and clinics is not recorded centrally (with the exception of birth and death events). The scale of this phenomena is hard to estimate, but the estimates of healthcare expenditure nationally indicate that every third pound spend on healthcare goes to the private healthcare market (Clarke et al., 2008; Department of Health, 2005a) (section 2.2). This bias could be termed cash and care bias. Popularity bias is when users with differential preference for a particular health service also have different attributes to those of the local population. Centripetal bias is similar, but is the instance, where users have preference for a particular clinician or family doctor. There may also be equity issues where some users advertently or inadvertently suffer reduced access to a service due to ethnicity, social status, neighbourhood, transport or economic resources; this is termed diagnostic/treatment access bias. Finally, the patterns of referrals are ordered according to their demand and severity. Local services typically deal with the bulk of low intervention services (primary care), and more complicated needs are referred to hospital-based specialists (secondary care), and the yet more complicated needs are dealt with by specialist units (tertiary care). A study relying on users of specific services may potentially be biased if the choice of reference population is not designed to counter such effects, i.e. the referral filter bias. 


\section{Information bias}

In the first instance we have considered the validity of the sampling frame and errors associated with selecting population subgroups. The following set of bias, information bias, concerns the quality of the data once collected.

For practical and conceptual reasons most exposures and outcomes are recorded as categories rather than point measurements. Health records are for example usually dependent on diagnoses separating patients into a 'non-diseased' versus 'diseased' category. There are strict quality control measures for the assays and other diagnostics used in medicine, which aim to minimise misclassification bias problems such as false positive and false negative (see also the discussion in section 5.3 on breast screening) (Muir Gray, 1996). Nonetheless one has to bear in mind that misclassification can cause bias in itself, non-differential misclassification bias, and more serious still, differential misclassification bias, where the effects of misclassification varies between different population groups. Misclassification bias also applies in exposure attributes, where it is related to concept of measurement bias; 'A major challenge in environmental epidemiology is to measure accurately each individual's exposure to hypothesized risk factors (i.e., the biologically relevant dose). This task is made very difficult by the lack of information about environmental sources of emission, the complex pattern of most long-term exposures, the individual's ignorance of previous opportunities for exposure, the lack of good biological indicators of exposure level, and the lack of sufficient resources to collect individual exposure data on large populations' (Morgenstern \& Thomas, 1993). The coding of data with deprivation indices is another example of the difficulties in classifying areas into discrete categories. Historically area deprivation has e.g. been based on the proportion of manual labourer occupancy, while the nature of occupation is not static and the underlying aspects of social status and material wealth are unlikely to be captured accurately (Noble et al., 2004) and so opens up possibilities of misclassification and bias towards or away from null. 
A bias that applies to screening in particular is detection bias whereby the probability of a positive test result increases with the level of effort. Often patients are 'filtered' for further examinations in a cascade of tests and as the sampling frame diminishes, the diagnosed group may no longer be a true picture of actual prevalence. Another problem applying to screening is lead-time bias. Screening campaigns detect tumours, but not how long a patient has lived with that tumour. This can have an impact of the estimates of survival and risk factors at different stages. To finish a long list of potential misclassification problems incomplete or inaccurate data recording could be added. This a particular issue in observational or secondary data analysis. A lot of efforts have recently gone into developing imputation techniques for missing data in multivariable data sets although similarly to ecological inference above it has to be acknowledged that this is a path to partial identification that needs to be backed up with analyses of sensitivity (see e.g. Carpenter et al., 2007).

Reporting bias is when data are collected the observer or doctor may be more inclined to test or diagnose a patient if there is a perceived match with a known or hypothetical risk factor; this is known as observer/interviewer bias. Where population health studies rely on survey data, there is also a risk of recall bias, which is a phenomenon where patients with e.g. lung cancer may be more forthcoming than others in remembering past exposures such as those pertaining to smoking habits. Reporting bias applies to all information volunteered by patients, whether they answer health related questions according to what they think the interviewer sees as the 'right' answer, i.e. the obsequiousness bias. Given an ordered list of levels many inadvertently choose the medium categories; a behaviour leading to the mode for mean bias. Once a case is diagnosed many from the same family will seek reassurance for what they rightly or wrongly infer as increased risk due to nature/nurture factors, i.e. the family aggregation bias. Health data also suffer from underreporting especially of conditions or exposures that are embarrassing or undesirable, e.g. mental or sexual health, hence the term unacceptable disease/exposure bias. 


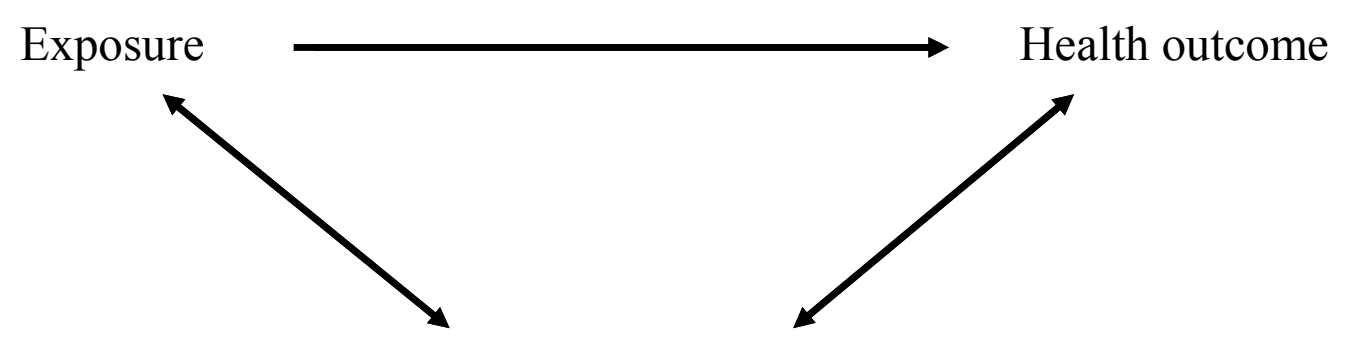

Confounder

Figure 19 Causation in studies of health outcomes. Arrows indicate 'causes' $(\rightarrow)$ and 'associated with' $(\leftrightarrow)$

Ecological bias or fallacy occurs 'when analyses based on grouped data lead to conclusions different from those based on individual data' (Waller \& Gotway, 2004). This problem is well known in geographical studies, where data are often aggregated at area level. In the worst cases ecological bias can lead to reversed causation. A geographical variant of the same problem is the Modifiable Areal Unit Problem (MAUP), whereby different results can be obtained by aggregating the same data in a different set of zones (zone effects) or to a higher level of aggregation in a geographical hierarchy, e.g. postcode $<$ ward $<$ county (scale effects) (Openshaw, 1984; , 1991). There is also a tendency for associations between variable to become stronger with aggregation to higher levels (the Gehlke-Biehl effect), which indicates a relationship between scale and uncoordinated variation ('noise'), although the effect remains to be fully explained (Waller \& Gotway, 2004).

\section{Confounding influences and effect modification}

Confounding is arguably the single greatest concern for studies of cause and effects. It happens when both cause and effect is associated with another non-causal variable, i.e. the confounder (Figure 19). The effects of confounders can be negated if these are identified and included in the inferential design. An example of confounding could be a study that claimed that 
smoking causes liver diseases without studying whether smoking in effect is associated with higher alcohol intake and that the actual causal pathway instead goes from high alcohol intake to liver disease.

Effect modification is where another variable modifies the effect of the main causal factor. An example of this could be if we in the previous example, would say, that smoking did indeed cause liver disease, but that the effect would be amplified or dampened by the level of another variable, i.e. alcohol intake. Effect modification can if not treated lead to faulty interpretation of cause and effect. One way to deal with effect modification is to stratify the study and measure the causal relationship at each level separately, e.g. for different levels of alcohol consumption in the example above.

\subsection{Neighbourhood constructs}

Many papers have been written about neighbourhood effects in health; especially from the mid 1990s (Cummins et al., 2007; Entwistle, 2007; Macintyre, 2007; Riva et al., 2007). The primary question has been whether so-called contextual effects measured at group level (the neighbourhood by some definition) were greater than the combined effects compounded by the individuals residing in that same area (compositional effects). Despite the intuitive lure of the idea about the 'local' environment the results of this type of studies vary to an extent that some have called their existence into question (Cummins, 2007; Pickett \& Pearl, 2001). What seems particularly difficult has been to find relevant and comparable scales for neighbourhood effects that would allow external validation of results from different studies (Cummins et al., 2007; Entwistle, 2007). Another issue seems to be that it is extremely difficult to fully dissociate individual effects from group effects due to the problems of self-selection (see section 7.3), the lack of lifecourse exposure information, the over-emphasis on local areas as proxy for (unmeasured) individual social ties and social networks, the moving-to-healthcare bias, the apparent correlations between areas of low income, high long-term disease prevalence and 
population density. Related to this, many GIS studies have counter-intuitively found that deprived areas generally have good geographical access to health promoting facilities such as healthcare services, healthy food, parks, etc. in terms of Euclidean distance, road distance or travel time (Cummins, 2007; Macintyre, 2007; Pearce et al., 2007). This could possibly be explained by the overlap between areas of concentrated deprivation and population density. Another explanation could be the pro-equity distribution of services by welfare governments (Pearce et al., 2007), something that seems more likely because most confirmatory studies of food and healthcare 'deserts' are from the USA, where communitarian aspects of urban planning and public service provision play a minor role as a consequence of neo-liberal and laissez-faire politics.

Studies of accessibility for different income and ethnic groups are nonetheless still important as there can be many local factors with implications for geographical and social accessibility (e.g. Brainard et al., 2006). 


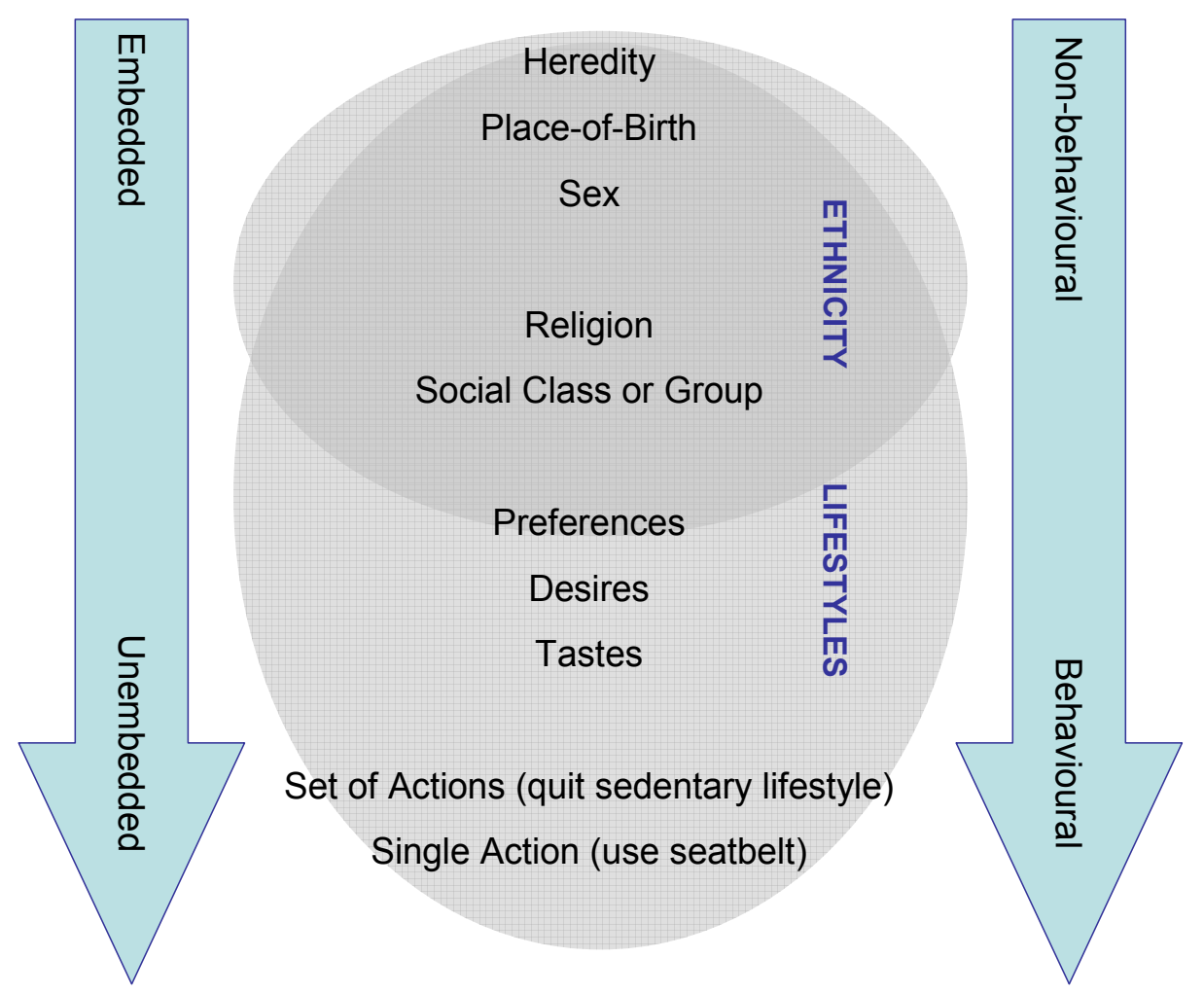

Figure 20 Identity, ethnicity, lifestyle and behaviour modifications

\subsection{Lifestyle construct}

This section aims to define lifestyles; what they are and how they may be measured and used in public health activities.

The lifestyle concept seems to have attracted several different meanings whether it is used casually with reference to anything from buying a packaged holiday to your marital status or more formally in the way it is used by either health or marketing professionals. Chaney (1996) defines lifestyles as 'patterns of action that differentiate people', where the pattern is characterised by 'a style, a manner, a way of using certain goods, places and times'. Chaney further argues that lifestyle 'help make sense of what people do, and why they do it, and what doing it means to them and others ...people use lifestyles in everyday life to identify and explain 
wider complexes of identity and affiliation'. It is interesting to note that lifestyles in this way becomes an integral part of people's identity much like the definition reached for ethnicity in chapter 5. Sociologists are interested in whether 'lifestyle' is 'free floating' something that can be 'donned and discarded at will' similar to Smedley and Smedley's (2005) definition of ethnicity as a purely social concept. Others will argue that even lifestyles to a degree are 'embedded in a social order' and that 'tastes' are emblems of social class or status (Clapham, 2006). Walters (2006) asserts that lifestyle consists of three elements beginning with 'c'; conditions, choice and cognition. He argues that lifestyle is pre-conditioned by 'internal (heredity', intelligence, temperament), external (family, peers, social class), and synergistic (reciprocal person x situation interactions) factors that increase or decrease people's behaviours but which do not determine their actions. Choice is the decision-making process by which options arising from the complex interplay of various life conditions are selected'. The third 'c' is for cognition is conditional for the creating an individual's alibi and belief system. Lifestyle in this view becomes a 'here I am, I am a modern and self-conscious individual. This is what I stand for. These are my beliefs and values. This is how I enjoy and justify my deserts ${ }^{10}$, etc.' Lifestyles perceived in this way falls in other words close to Jung's persona concept (Jung, 1928, p.305). Using Walters' broader definition of lifestyle thus encompasses almost any identity forming attributes including heredity and ethnicity (Figure 20). In Figure 20 individual attributes are arranged from top to bottom on a scale from non-behavioural/embedded to behavioural/un-embedded attributes. Embeddedness is meant to represent how easy it would be for someone to change this attribute. Change is of particular interest to the health promoting campaigns or so-called behaviour modification programmes (Holland, 2007; Seedhouse, 2004). Lifestyle in this context becomes a question of which attributes are modifiable by voluntary action. 'Using a seatbelt', for instance, is an example

\footnotetext{
${ }^{9}$ We can take 'heredity' to span both genotype and phenotype in this context

${ }^{10}$ To take a deontological angle (chapter 1)
} 
of a campaign aim that requires relatively little of a patient, because it involves just one action (Figure 20). Whilst quitting a sedentary lifestyle is higher on the list, because it is more involved, i.e. the patient has to exercise more, change diet, maybe quit smoking and cut down on alcohol consumption. Attacking someone's lifestyle affects a whole set of attributes that can be said to be part of that person's identity and hence be more problematic in intervention terms. If, for example, the patient's idea of a 'good life' means meeting up with friends for a drink after work or smoking with colleagues, then behaviour modification could arguably have negative consequences for that patient's well-being that lie beyond those of better physical health.

Acculturalisation would mean, as discussed in section 5.3 on breast screening uptake, that an individual with time would move from adherence to the less amenable attributes in the top half of Figure 20 (ethnicity) and gradually adopt more mainstream lifestyles of the host country in the lower half of the figure (Van Oudenhoven et al., 2006). In a multicultural society however some ethnic groups may instead prefer to integrate rather than assimilate and thus keep parts of their heritage culture; e.g. Muslims in the UK have religion in common, but at the same time enjoy a wide range of different lifestyles. Other complementary observations should be that ethnicity is not simply synonymous with any 'hard-to-change' or indeed 'up-for-change' lifestyle attributes. There are many examples of overlap between healthy lifestyles and ethnicity (see e.g. Shetty, 2001 on 'ancestral' versus adopted dietary habits).

In summary, we can say that lifestyle encompasses almost any aspect of human behaviour and identity (Chaney, 1996); both of which to a large extend are conditioned by the environment. For health promotion it is useful to project lifestyle attributes on a scale from the non-behavioural, e.g. genotype, to the less embedded behavioural attributes, e.g. preferences (Holland, 2007). Ethnicity in many ways represents the more embedded attributes, such as religion, where acculturalisation is likely to lead to more mainstream lifestyles (Van Oudenhoven et al., 2006). This is of course not to suggest that this is a straight and clearly demarcated path; the unfolding of 
multicultural societies has shown that people of the same ethnic group may adopt many different lifestyles. Lifestyle modification is controversial because it aims to correct what essentially is a suite of identity-forming attributes, 'the way we are and see ourselves'. Health promotion is for that reason often most successful when it tackles single action issues, e.g. remembering to wear seatbelt, putting babies to sleep on their back or drink-free driving (Holland, 2007; Seedhouse, 2004). Changing entire lifestyles involves many different attributes and, although they may seem 'modifiable', preferences, desires or tastes can be interlocked with an individual's identity, belief system or worldview (Walters, 2006). It is for that reason all the more important to involve patients actively in their own 'cure' for the success of such programmes (Holland, 2007; Seedhouse, 2004).

The next section will deal more specifically with how lifestyles can be measured and used in public health campaigns. The section will begin with an analysis of how marketing professionals view lifestyles in contrast to the socio-medical model presented so far.

\section{Lifestyle indicators and the lifestyles-neighbourhood 'shunt'}

Comprehensive and updated lifestyle information would be expensive to collect and its storage would pose threats to individual privacy. We, as citizens, would also have to agree on which indicators data should be collected and for which purposes. The commercial purpose however is clear: to maximise profit from direct marketing. Consumer data from customer databases and industry surveys has thus become a valuable commodity for the marketing industry; the 'who is buying what and where, their disposable incomes and under which circumstances and conditions they will act'? Lifestyle information is also collected in a more scientific fashion through governmental surveys, e.g. the Health Survey for England (HSE) or British Household Panel Survey. These data sources allow more detailed questions about the possible interactions between socio-economic circumstances, lifestyle attributes and health 
outcomes in the population. The surveys are designed around a minimum sample of participants balancing costs and external validity. The HSE, for instance, interviewed and medically examined 21,000 individuals for their annual survey in 2006 (Office for National Statistics, 2008b). Apart from the general health trends many local planning authorities - and commercial marketers have a keen interest in whether this anonymous and aspatial information can be disaggregated, modelled and mapped for local areas. There are different techniques for this, synthetic estimates, micro-simulation and geodemographics. Geoemographics has garnered most interest and is now distributed in many commercial formats as well as the Census classifications disseminated by Office for National Statistics (ONS). The following will briefly explain the fundamentals of geodemographic systems and how they may be used for estimating lifestyle information in local contexts.

If health promoters are the magpies of the health profession (see chapter 1), then the creators of geodemographic systems must be the magpies of the marketing profession. No survey, high street interview or customer database seems unfit for geodemographic data mining. The addresses of participants are coded with neighbourhood type and turned into attribute propensities revealing which behaviours can be associated with the dwellers of different neighbourhood types. This is the lifestyles-neighbourhood 'shunt'. The term shunt is used to indicate that knowledge is here created in a very simple way that only requires few and simple mathematical steps. It is as if information is moved back and forward between the two constructs, lifestyles and neighbourhoods, as through a bypass. The lifestyles-neighbourhood shunt is, in simple terms, dependent on three things; a) address matching to a geographic base file, appended with b) base population estimates, and coded with c) a neighbourhood type classification. All of these services are now supported by government agencies with Census output areas (OA) as the minimum geographic level of aggregation (Vickers et al., 2005). 
In order to gain more insight into this to date mainly commercially driven technology the next subsection will begin with some examples of lifestyles and consumer segmentation systems.

\section{From lifestyles segmentation systems to geodemographics}

Marketing companies have created their own systems for segmenting customers, households, postcodes or other geographical units. USA Today, the biggest daily newspaper in the USA, e.g. offers their online advertisers a content-based readership classification (Figure 21) (USA Today, 2008).

Figure 21 USATODAY.COM content-based readership classification (USA Today, 2008)

A. Armchair Athletes - male readers between the ages of 21 and 30 who have been to Sports in the past 30 days

B. Bulls and Bears - any reader who has used Investor Research services within the Marketwatch cobrand, maintains a portfolio on USATODAY.com, or has checked stock quotes in the past 30 days.

C. Lookers and Bookers - any visitor who has visited Travel (excluding travel-related news stories) in the past 30 days.

D. Early Adopters - any reader who has visited Tech Reviews or checked wireless hot spot locator in the past 30 days.

E. Tire Kickers - any visitor who has visited the Test Drive Auto Review column or any of the Cars.com co-branded car search engines within the past 30 days.

F. Create Your Own Segment - advertisers can combine the specific behavior and/or demographics that they are targeting into one custom segment.

Content-based classifications typically classify textual or other amorphous information such as trails of web pages visited by a consumer. They are part of a growing trend, because text, in the digital age, has remained the most common vehicle for exchange of information (Witten \& Frank, 2005). Tags and hyperlinks are the most visible outcomes of the efforts to create a common reference frame for the meaning of words in electronic content, the semantic web (Berners-Lee et al., 2001). It is the digital traces of 'interesting' words that helps create these classifications. As with any other classification it is also the classification owners that define the 'interestingness' of different words, i.e. the classification criteria. 
The next level of generalisation can be at household level and is e.g. utilised in the US Niches

Lifestyles system with 25 types - one for each letter of the alphabet from Already Affluent to

Zero Mobility (Figure 22).

The most commonly used systems for market segmentation in UK are based on the postcode system, e.g. the 11 Mosaic UK Groups in the Mosaic system (Figure 23) (Harris et al., 2005).

Figure 22 Household segmentation system, Niches Lifestyle system. named from A-Z (Bickert 1995)

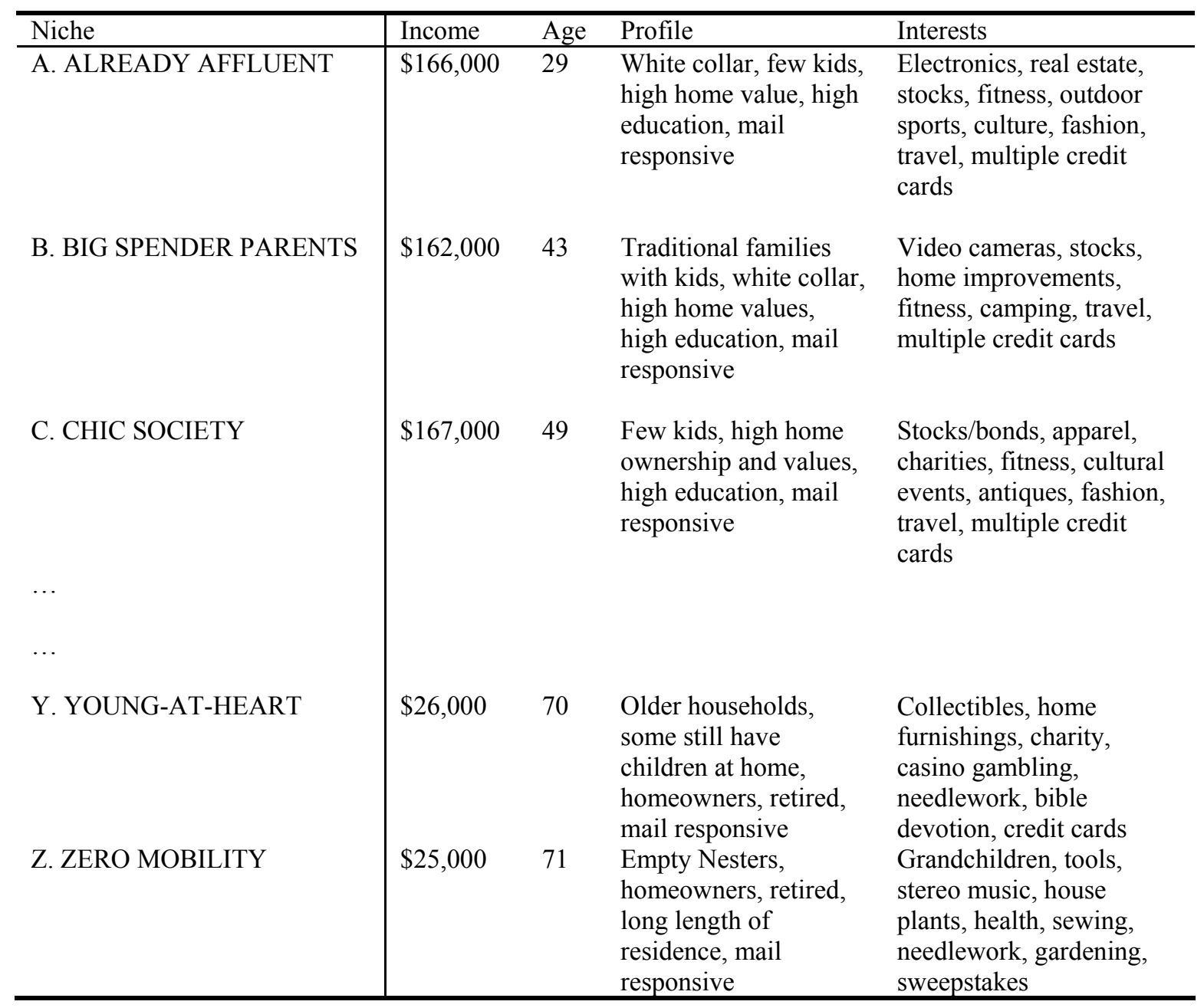


Lifestyle segmentation systems based on geographical units (postcodes in the case of Mosaic UK) are commonly referred to as geodemographic systems. Their value for marketing is based on the simple assertion that consumers with similar lifestyles cluster together in similar types of housing, streets and neighbourhoods (Flowerdew \& Leventhal, 1998). The advantage of using the lifestyles-neighbourhood shunt is that it opens up for loose coupling with a greater variety of data sources. If we compare it to USA Today's content-based classification (Figure 21) it is clear that we can gain specific and timely insight into the way readers surf their web content, but other individual information would be more problematic to append due to data protection issues. Once neighbourhoods, however, are coded in e.g. a Census classification, then any survey or data source can be used to describe that classification as long as it is geo-referenced, i.e. the process of creating a geodemographic system.

\section{Figure 23 Mosaic UK consumer postcode segmentation system}

A. Symbols of Success - People with rewarding careers who live in sought after locations, affording luxuries and premium quality products

B. Happy Families - Families with focus on career and home, mostly younger age groups now raising children.

C. Suburban Comfort - Families who are successfully established in comfortable, mature homes. Children are growing up and finances are easier.

D. Ties of Community - People living in close-knit inner city and manufacturing town communities, responsible workers with unsophisticated tastes.

E. Urban Intelligence - Young, single and mostly well-educated, these people are cosmopolitan in tastes and liberal in attitudes

F. Welfare Borderline - People who are struggling to achieve rewards and are mostly reliant on the council for accommodation and benefits

G. Municipal Dependency - Families on lower incomes who often live in large council estates where there is little owner-occupation

H. Blue Collar Enterprise - People who though not well-educated are practical and enterprising and may well have exercised their right to buy.

I. Twilight Subsistence - Elderly people subsisting on meagre incomes in council accommodation.

J. Grey Perspectives - Independent pensioners living in their own homes who are relatively active in their lifestyles

K. Rural Isolation - People living deep in the countryside in small communities little influenced by influx of urban commuters

The time scale for which the different segmentation systems are created and used is interesting in itself. In USA Today's content-based customer segmentation system you are only identified by your activities within the last month. If for some reason you do not browse their webpage for over 
a month, then you no longer 'exist'; i.e. your identity is temporary. In contrast, if someone bought a sports car or went to hospital once, that may influence decisions based on geodemographic profiling for several years, even though the consumer may long have moved away.

\subsection{Assumptions in geodemographics}

The basic principles behind geodemographics has already been described in chapter 4 , the previous section and are comprehensively covered in recent publications (Harris et al., 2005; Sleight, 2004). This section will look closer at the assumptions made when creating and using a geodemographic system for targeting.

1) Formalisation assumption - it is assumed that neighbourhoods can be adequately characterised by a number of indicators, usually Census variables, concerning tenure, occupation, unemployment, ethnic composition, car ownership, etc. This is an assumption required for any construct (Pedhazur \& Pedhazur Schmelkin, 1991; Schuurman, 2006) and which is also necessary for the clustering of neighbourhoods in an n-dimensional attribute space through e.g. the k-means clustering routine (Harris et al., 2005).

2) Attribute space assumption - it is assumed that socio-economic proximity is a more relevant reference frame for studies of group behaviours than locational proximity (Webber \& Longley, 2003), i.e. that geodemographics is an alternative conceptual model to the current practice of aggregating data in rigid and potentially more heterogeneous administrative units and that it, as a consequence, addresses scale effects in geographical analysis (Openshaw, 1984) in a superior manner by aggregating many smaller but less heterogeneous geographic units (Webber \& Longley, 2003).

3) Ecological assumption - it is assumed that dwellers of similar neighbourhoods based on the clustering variables (socio-economic proximity) also have similar behaviours. Exposure and outcome are correlated at group level, although we have no information about the constituting 
individual level associations. It is in other words an unidentified problem (Manski, 1995); a problem for which there are no solutions.

A critical aspect of this problem is scale. Aggregating neighbourhood data by socio-economic proximity has the advantage that the limit for unit population size can be lowered, i.e. where robust baseline estimates before could only be obtained at local authority level (average population of approximately 300,000), a geodemographic analysis would aggregate tens of thousands of postcodes each with roughly 50 inhabitants. Several studies of geodemographics have shown a capability for these systems to successfully expose the macro-scale social arrangement in a more detailed fashion than is achieved with e.g. deprivation scores of larger administrative areas. Geodemographic neighbourhood classifications are the result of a multidimensional clustering process and as a consequence it may be possible to distinguish between e.g. a postcode with many elderly on low income living in publicly rented flats on one side of a street and the stylishly converted warehouse accommodating high-earning singles on the other side of the street (see examples in section 7.5). Geodemographics may also reveal more subtle differences, e.g. in the analysis of equity of public services at a national level (Harris et al., 2005). It can in other words be a useful instrument for the evaluation of macro-scale egalitarian policies. Local health authorities are nevertheless less likely to influence macro-economic factors, whereas individual health behaviours may be easier targets (chapter 3). Compared to deprivation scores, however, geodemographics does offer more detailed descriptions of neighbourhoods - albeit a 'statistical' neighbourhood rather than a 'de facto' one.

In chapter 3 behaviours of groups were characterised as correlated or endogenous. A type of preference may be thus determined by environmental pressures (correlated) - 'other people would have chosen the same under those circumstances' or social pressures (endogenous) - 'everyone around here does that'. The ecological fallacy seems a greater problem with the latter type of 
assumption. An example; aspatial analysis of area deprivation is ubiquitous in studies of health outcomes - it is almost a convention. The emphasis on social causation (i.e. that adverse socioeconomic circumstances cause poor health outcomes) is heavily implied in these studies. Teenage pregnancy rates, for instance, are typically presented scattered against multiple deprivation score for each local authority (Social Exclusion Unit, 1999). Is a positive correlation indicative of correlated or endogenous effects or both? There seems to be a preference for correlated effects and less attention to the endogenous (see section 6.4), although the latter - as concluded in chapter 3 - may have a greater potential for social marketing. Would geodemographics be a better reference frame for this type of studies? Webber and Longley (2003) suggested that the socioeconomic proximity (that geodemographics offers) potentially would be a more relevant framework than the current practice in government institutions (e.g. Office for National Statistics or the Public Health Observatories) to use administrative areas. In other words whether socioeconomic proximity is more relevant than locational proximity (Webber \& Longley, 2003). The accompanying assertion for the targeting of public health campaigns would be that health behaviours in general would be closer associated with correlating socio-economic variables than with place-specific factors. This seems plausible although initial geodemographic analyses of the individual level health behaviours studied in chapter 5, 'light' frequent use of A\&E, GP subscription, or breast screening uptake, failed to discern differences among neighbourhood types that were deemed sufficiently specific as a basis for local health campaigns (section 5.4). Does this raise a need for new geodemographic systems that a) incorporate the variables used in the individual level analysis, e.g. names-based ethnicity? Or b) focus on specific geographical regions rather than a country as a whole? Or c) whether routinely collected data on demands, for e.g. healthcare, would prove more timely and relevant as the attribute space for clustering of neighbourhoods? The two last questions will be addressed in the chapter 8 and 9 . 


\subsection{Social critique of geodemographics}

Geodemographics has been subject for social critique (Burrows \& Gane, 2006; Goss, 1995; , 2003; Graham, 2005; Philips \& Curry, 2003); which is essentially summed up in the following quote; '[geodemographics] is (re)producing the socio-spatial differentiation of society and reinforces past behaviours in particular places' (Goss, 2003). The use of geodemographics, in other words, 'frames' and restrict people and herds them towards pre-defined consumer stereotypes. Let us put this statement into proportion.

First, we are bombarded with advertising throughout the day, where we shop, commute, from every media including the internet. Especially on the internet we see ever more sophisticated ways of targeted advertising, e.g. in the adverts selected on the basis of the content of your searches or even of your personal emails. Despite the Orwellian overtones of 'thought policing' this type of advertising must - considering the popularity of these services (e.g. Google) - seem acceptable or at least bearable. The targeted mail on your doormat in contrast seems somewhat less intrusive. This is not intended as a perfect rebuttal of the concerns over social sorting and privacy intrusion expressed by Goss and others, but to note that neighbourhoods are not the 'stable containers for certain populations and activities' assumed by creators and early critiques of geodemographics, but 'fluid, both temporally and spatially, ... products of the actions of their inhabitants. ... created by the behavior of individual inhabitants, the goal [hence] becomes to influence those behaviors through direct, persuasive appeals' (Philips \& Curry, 2003). In this light the fears and criticism of geodemographics seems overstated, while systematic and centralised surveillance of the individual behaviours remains a social concern.

Second, Goss's statement implies that the consumer is a victim of a non-egalitarian pure market-oriented society, a loser in the 'ecological competition for urban space between social groups' (Goss, 1995) and not someone intelligent enough to make qualitative choices about assorted commercial mail shots. Ironically, it is Goss that seem most convinced of the notion that 
'where you live is everything', whereas marketers would use a geodemographic system with the calculated loss due to its relative inefficiency - spatially as well as temporally (Longley, 2005).

Third, fears that geodemographics would be adding to 'surveillance creep' and a push towards a 'panoptical' society again seems overstated (Lyon, 2006; Philips \& Curry, 2003). Graham (2005) concludes 'Spaces which escape the reach of regressive software sorting systems do and will remain. ... Software- sorting techniques also offer much potential for progressive and empowering policy innovations'. It seems that geodemographics is perceived by its later critics (Graham, 2005; Philips \& Curry, 2003) as sufficiently blunt not to be intrusive, but on the other hand sufficiently specific to be used in neighbourhood policies. 


\section{Chapter 8 Neighbourhood classification based on Census variables}

The growing demand upon hospital and healthcare resources follows a general trend across the economically developed world as a consequence of increasingly sedentary lifestyles, rising calorie supply per capita and ageing population (Bleich et al., 2008; Bodenheimer et al., 2002; Department of Health, 2004b; Saxena et al., 2006; Wagner, 1998). Hospital admissions are of prime concern due to both associated costs and social implications. Although hospital admissions only account for $19 \%$ of all recorded contacts, they account for $58 \%$ of the NHS expenditure (Talbot-Smith \& Pollock, 2006) (Figure 24). Patients with long term or chronic diseases have the greatest needs; they only represent $5-10 \%$ of patients, but account for up to $55 \%$ of hospital bed days (Department of Health, 2004b).

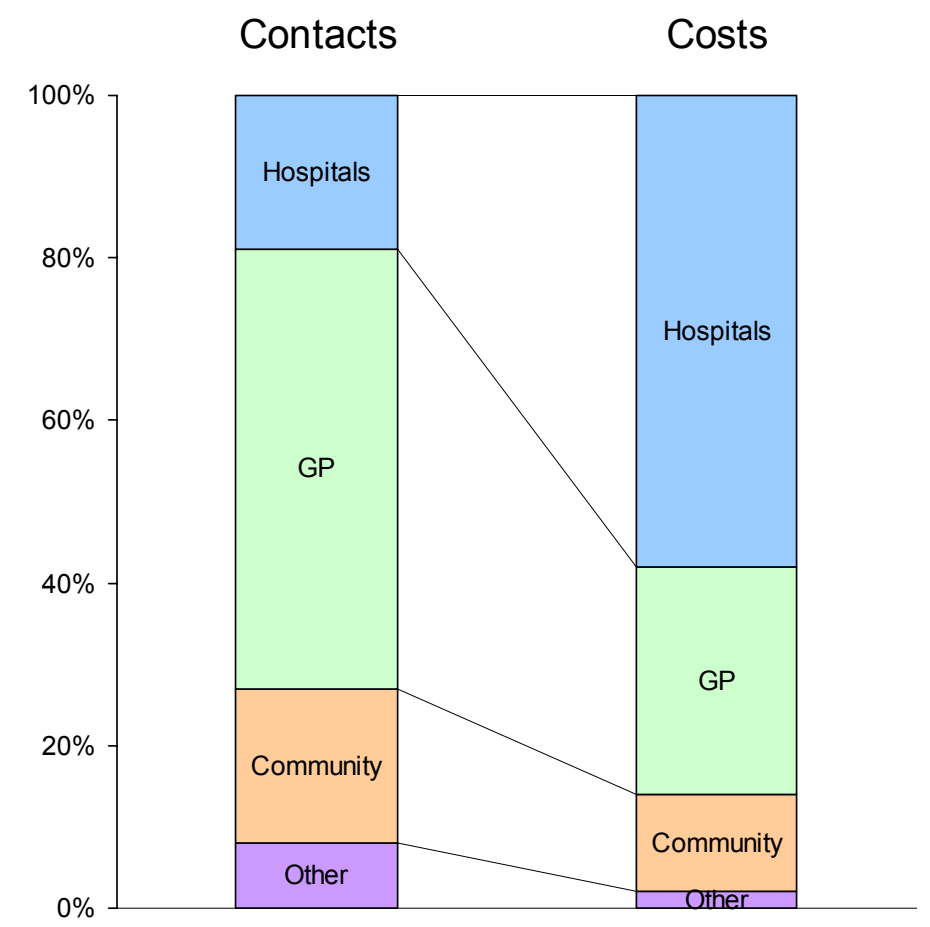

Figure 24 Contacts versus Costs in NHS across service sectors (data from Talbot-Smith \& Pollock, 2006) 
How do we study the societal causes and effects of the rising prevalence of chronic diseases? Geodemographics offers easily accessible means of mapping needs and potential causes across a wide range of socio-demographic variables. In the following geo-coded hospital admission data from Hospital Episode Statistics (HES) will be used to map and evaluate the potentials of geodemographic data mining. The structure of the chapter is a follows;

1) Problem definition - A discussion of the problems associated with the current geodemographic systems for health applications. This section is followed by three empirical examples; i.e.

2) London Output Area Classification (LOAC) - The development of regional neighbourhood classification based on Census data

3) Evaluation of targeting efficiency in a health applications with hospital demand data across a range of commercial and publicly available classifications

4) The potential utility of age- and sex-standardising geodemographic analysis of hospital demand data

\subsection{Problem definition}

Geodemographics offers a promising way to integrate, model and map healthcare needs and other health indicators that are useful for the geographic targeting of public health campaigns (chapter 7). Yet reports about this type of applications seem sporadic and just as promotional in 2004 (Department of Health, 2004c) as they were in 1985 (Speller \& Hale, 1985). One of the major obstacles has presumably been the lack of validation beyond the observation that it works sufficiently to be of interest for direct marketing (Vickers et al., 2005). Office for National Statistics (ONS) has built publicly available geodemographic systems from Census data since the mid-1990s, but again reports of health applications have been sporadic (Openshaw, 1995). The latest Census classification, the 2001 Census output area classification (OAC), however has been promoted in a more sustained manner, e.g. by appending it to the national geographical look-up 
table, National Statistics Postcode Directory, and several national surveys. The 'open source' approach also offers opportunities for evaluating the system or modifying it for new purposes (Singleton \& Longley, 2008).

OAC consists of a three-tier hierarchy with 7 Supergroups subdivided into 21 Groups again subdivided into 52 Subgroups. Mapping OAC for Greater London reveals a pattern with large swathes of Inner London belonging to single segment, the "Multicultural" Supergroup, and many other areas forming a mosaic of only sparsely represented segments (Figure 25).

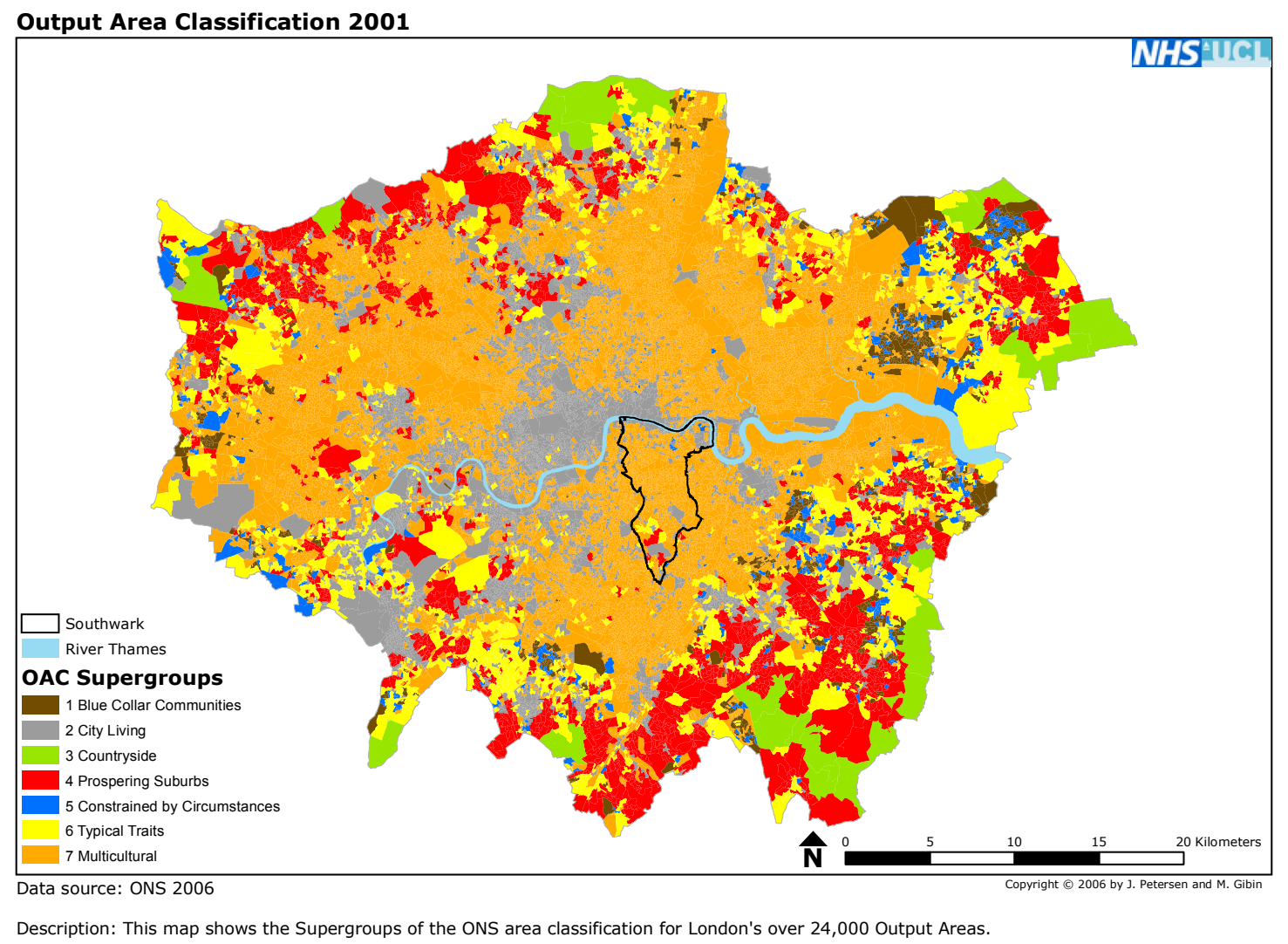

Figure 25 Output Area Classification mapped for Greater London (OAC Supergroups)

This leaves an inner city borough such as Southwark with $86 \%$ of their 319,000 registered patients in just one OAC Supergroup (Table 14). The dominance of a single segment continues in the Group classification - one tier down from Supergroups. The majority of patients here resides 
in "Afro-Caribbean Communities". Segmentation with the finest and third tier segments, the Subgroups, finally reveals that $44 \%$ of patients reside in just one unnamed Subgroup (data not shown).

Table 14 Output Area Classification profile of patients registered in Southwark PCT, April 2006.

\begin{tabular}{|c|c|c|c|c|}
\hline OAC Supergroup & Freq. & Percent & Mean IMD score & Typical Census attributes \\
\hline 1 Blue Collar Communities & 7 & $<0.1$ & 28 & $\begin{array}{l}\text { Terraced housing, renting } \\
\text { publicly }\end{array}$ \\
\hline 2 City Living & 35,450 & 11.1 & 25 & $\begin{array}{l}\text { Higher education } \\
\text { qualifications, single person } \\
\text { household (not pensioner), } \\
\text { born abroad, renting privately, } \\
\text { all flats }\end{array}$ \\
\hline 3 Countryside & 0 & 0 & - & $\begin{array}{l}2+\text { cars per household, } \\
\text { working from home, } \\
\text { agriculture/fishing } \\
\text { employment, detached } \\
\text { housing }\end{array}$ \\
\hline 4 Prospering Suburbs & 2,266 & 0.7 & 17 & $\begin{array}{l}2+\text { cars per household, } \\
\text { detached housing }\end{array}$ \\
\hline 5 Constrained by Circumstances & 2,297 & 0.7 & 43 & All flats, renting publicly \\
\hline 6 Typical Traits & 3,743 & 1.2 & 17 & Terraced housing \\
\hline 7 Multicultural & 275,488 & 86.3 & 37 & $\begin{array}{l}\text { Renting privately or publicly, } \\
\text { commuting to work on public } \\
\text { transport, all flats, born } \\
\text { abroad, South Asian or Black } \\
\text { ethnic background }\end{array}$ \\
\hline Total & 319,251 & 100.0 & 36 & \\
\hline
\end{tabular}

Sources: ONS, Indices of Multiple Deprivation (IMD) 2004 score (Noble et al., 2004)

A similar picture repeats itself with the commercial postcode level segmentation systems, e.g. Mosaic UK (Table 15). Although this system exhibit greater differentiation of neighbourhoods the majority of groups contain fewer than $1 \%$ of patient and it still contains a very large dominant neighbourhood segment with $49 \%$ of all patients, the "Welfare Borderline" Group (Table 15), and $48 \%$ in the second tier, "Metro-Multiculture" segment (data not shown). The lack of specificity in these classifications makes them less suitable for targeted interventions and this is the motivation 
Table 15 Mosaic UK profile of patients registered in Southwark PCT, April 2006

\begin{tabular}{|c|c|c|c|c|}
\hline Mosaic UK Group & Freq. & Percent & Mean IMD score & Description \\
\hline $\begin{array}{l}\text { A. Symbols of } \\
\text { Success }\end{array}$ & 13,276 & 4.2 & 19 & $\begin{array}{l}\text { People with rewarding careers who live } \\
\text { in sought after locations, affording } \\
\text { luxuries and premium quality products }\end{array}$ \\
\hline B. Happy Families & 1,162 & 0.4 & 39 & $\begin{array}{l}\text { Families with focus on career and home, } \\
\text { mostly younger age groups now raising } \\
\text { children }\end{array}$ \\
\hline $\begin{array}{l}\text { C. Suburban } \\
\text { Comfort }\end{array}$ & 2,885 & 0.9 & 22 & $\begin{array}{l}\text { Families who are successfully } \\
\text { established in comfortable, mature } \\
\text { homes. Children are growing up and } \\
\text { finances are easier }\end{array}$ \\
\hline $\begin{array}{l}\text { D. Ties of } \\
\text { Community }\end{array}$ & 29,251 & 9.2 & 35 & $\begin{array}{l}\text { People living in close-knit inner city and } \\
\text { manufacturing town communities, } \\
\text { responsible workers with unsophisticated } \\
\text { tastes. }\end{array}$ \\
\hline $\begin{array}{l}\text { E. Urban } \\
\text { Intelligence }\end{array}$ & 104,026 & 32.6 & 30 & $\begin{array}{l}\text { Young, single and mostly well-educated, } \\
\text { these people are cosmopolitan in tastes } \\
\text { and liberal in attitudes }\end{array}$ \\
\hline $\begin{array}{l}\text { F. Welfare } \\
\text { Borderline }\end{array}$ & 156,554 & 49.0 & 42 & $\begin{array}{l}\text { People who are struggling to achieve } \\
\text { rewards and are mostly reliant on the } \\
\text { council for accommodation and benefits }\end{array}$ \\
\hline $\begin{array}{l}\text { G. Municipal } \\
\text { Dependency }\end{array}$ & 372 & 0.1 & 44 & $\begin{array}{l}\text { Families on lower incomes who often } \\
\text { live in large council estates where there } \\
\text { is little owner-occupation }\end{array}$ \\
\hline $\begin{array}{l}\text { H. Blue Collar } \\
\text { Enterprise }\end{array}$ & 2,327 & 0.7 & 39 & $\begin{array}{l}\text { People who though not well-educated } \\
\text { are practical and enterprising and may } \\
\text { well have exercised their right to buy }\end{array}$ \\
\hline $\begin{array}{l}\text { I. Twilight } \\
\text { Subsistence }\end{array}$ & 3,593 & 1.1 & 39 & $\begin{array}{l}\text { Elderly people subsisting on meager } \\
\text { incomes in council accommodation. }\end{array}$ \\
\hline $\begin{array}{l}\text { J. Grey } \\
\text { Perspectives }\end{array}$ & 2,416 & 0.8 & 21 & $\begin{array}{l}\text { Independent pensioners living in their } \\
\text { own homes who are relatively active in } \\
\text { their lifestyles }\end{array}$ \\
\hline K. Rural Isolation & 0 & 0.0 & - & $\begin{array}{l}\text { People living deep in the countryside in } \\
\text { small communities little influenced by } \\
\text { influx of urban commuters }\end{array}$ \\
\hline L. Unclassified & 3,389 & 1.1 & 34 & Unclassified localities \\
\hline Total & 319,251 & 100.0 & 36 & \\
\hline
\end{tabular}

Sources: Experian Ltd (Notingham), Indices of Multiple Deprivation (IMD) 2004 score (Noble et al., 2004) 
to improve on the existing Census classification, OAC. The problem of specificity will in the following be discussed in a more general context in order to identify particular shortcomings that a modified version of the Census classification should address.

First, most of the geodemographic systems are based on clustering of attribute variables with no acknowledgement of the relative geographical location of localities and as a result they contain residual regional variation that may erode construct validity, i.e. the regionality problem (Sleight, 2004). Second, all national classifications tend to be represented regionally by one dominant neighbourhood type and a number of other types of exponentially falling representation, the fragment problem (Batty, 2006). This causes problems for the robustness of the imputation of attribute values, where base population numbers are low. The fragment problem is exacerbated for health applications due to the way many health problems follow of a strong age gradient. Place effects may hence be confounded by differences in population age structure alone. To avoid this health outcomes are usually standardised by age and sex. These standardisations however require further stratification of base population numbers that in many cases are already very small (see e.g. Table 14).

The regionality cum fragment problem is manifested in attempts to generalise attribute space, where e.g. Inner London boroughs are distinctly different from most other locations in Great Britain on a multiple of ethnic and socio-economic factors. The clustering technique, e.g. kmeans, searches at random for the optimal position of a given number of 'seeds', $\mathrm{k}$, that minimises the distance each neighbourhood has to the nearest seed when ordinated into a multidimensional attribute space spanned by the clustering variables. This process is sensitive to two 'forces'; a) proximity in attribute space; and b) outliers. In the case of Inner London, localities appear to cluster together more by being different from everywhere else (outliers) than by being uniquely similar (proximity).

There seems to be two immediate solutions to this problem. One solution could be to segment clusters further by clustering the clusters recursively. This is the most common route of creating 
differentiation in geodemographic systems (Harris et al., 2005; Sleight, 2004). The problem with this procedure on the other hand is that it, if anything, exacerbates the fragment problem, i.e. even greater heterogeneity in base population sizes. Another solution, which will be pursued here, is to create a regional classification. Hypothetically a regional classification would achieve greater differentiation and more evenness in base population structure simply by narrowing the attribute space to contain data from this region only; in this case Greater London.

In order to evaluate the potential utility of geodemographics in healthcare applications data on hospital episodes (Hospital Episode Statistics or HES) were obtained for Greater London. This data set will be used in a comparison of commercially and publicly available geodemographic systems with regards to performance indicators of targeting efficiency. The data will also be used for a study of potential benefits of age-standardisation of hospital admission ratios for geodemographic clusters.

\subsection{Methods}

\section{Selection of chronic disease indicators}

Hospital episodes data for Greater London, 2001-2004, were obtained with age, sex, residential postcode and primary diagnosis. Ethical approval was obtained through Bromley Local Research Ethical Committee and Patient Information Advisory Group (PIAG).

In approximately $10 \%$ of admissions patients are re-diagnosed at hospital and consequently referred on to a second consultant in a different speciality. In these cases only the first diagnosis was kept (first 'episode' in the 'spell' to follow the HES terminology). The diagnoses (ICD10 four-character system) were categorised into chronic disease indicators. These indicators have been selected as the most frequent chronic diseases (Department of Health, 2004b; , 2005b; Office for National Statistics, 2000); see Table 16. 
Table 16 Number of chronic disease admissions to hospital for residents of Greater London, Hospital Episode Statistics 2001-2004

\begin{tabular}{llc}
\hline Label & Chronic disease indicator & Admissions \\
\hline Angina & Angina pectoris (I20,I25) & 119,538 \\
breastca & Breast cancer (C50,D05) & 91,026 \\
bowelcan & Colorectal cancer (C17-C21) & 87,988 \\
chestpai & All chest pain (R073-074,R101) & 85,861 \\
backpain & Back pain (M50-M54) & 55,713 \\
mentalhe & Mental health (F20-F48) & 54,180 \\
arthriti & All arthroses (M15-M19) & 51,332 \\
leukaemi & Leukaemia (C91-C95) & 49,963 \\
copd & COPD (J40-J44) & 49,751 \\
stroke & Stroke (I60-I69) & 43,930 \\
lungcanc & Lung cancer (C33-C34) & 43,774 \\
asthma & Asthma (J45-J46) & 36,573 \\
heartfai & Congestive heart failure (I50) & 32,759 \\
heartatt & Acute myocardial infarction (I21-I24) & 31,458 \\
gallstone & Cholelithiasis (K80) & 29,145 \\
Tbi & Traumatic brain injury & 26,333 \\
diabetes & Diabetes (E10-E14) & 25,508 \\
skincanc & Skin cancer (C43-C44) & 23,663 \\
epilepsy & Epilepsy (G40-G41) & 19,087 \\
prostcanc & Prostate cancer (C61) & 16,669 \\
cervcanc & Cervical cancer (C53,D06) & 10,620 \\
\hline & Total & 984,871 \\
\hline Source: Depertment &
\end{tabular}

Source: Depertment of Health

\section{Modification of the Census Output Area Classification for Greater London}

The Output Area Classification (OAC) is based on a selection of 41 Census 2001 variables ranging from age to ethnicity, family structure, tenure, education, occupation, transportation, and health (Vickers et al., 2005) (Table 17). Localities (Census output areas in Great Britain) are first divided into 7 OAC Supergroups using the k-means clustering algorithm. The stopping rule for the generation of these Supergroups is guided by mean centroid distance, which as a statistic that decreases with increasing number of seeds, $\mathrm{k}$. Abrupt changes in this statistic is taken as an indication of a 'naturally' occurring number of clusters. The final number of clusters are also guided by a more pragmatic consideration, i.e. the fact that it is inherently difficult for end users to differentiate between much more than seven classes when the classification is mapped (Callingham, 2006). 
Table 17 Census clustering variables

\begin{tabular}{ll}
\hline Clustering variables from Census 2001 used in OAC and LOAC classifications \\
Age $0-4$ & Rooms per household \\
Age $5-14$ & People per room \\
Age $25-44$ & HE qualifications \\
Age $45-64$ & Routine/Semi-Routine occupation \\
Age $65+$ & $2+$ Car household \\
Indian/Pakistani/Bangladeshi & Public transport to work \\
Black African & Work from home \\
Black Caribbean or Black Other & Long Term Limiting Illness \\
Born outside UK & (Standardised Illness Ratio) \\
Population density & Provide unpaid care \\
Divorced & Students (full time) \\
Single person household (not pensioner) & Unemployed \\
Single pensioner household (pensioner)* & Working part-time \\
Lone parent household & Economically inactive looking after family \\
Two adult no children & Agriculture/fishing employment \\
Households with non-dependent children & Mining/quarrying/construction employment \\
Rent (public) & Manufacturing employment \\
Rent (private) & Hotel \& catering employment \\
Terraced Housing & Health/social work employment \\
Detached Housing & Financial intermediation employment \\
All Flats & Wholesale/retail employment \\
No central heating & \\
*) labelled "singlepl" to distinguish it from the non-pensioner variable ("singlepe"). Source: Vickers et al. 2005 &
\end{tabular}

In order to explore whether greater differentiation could be obtained for London

neighbourhoods, the Census data were re-clustered according to the OAC methodology, but with three modifications to form an alternative regional classification, London Output Area

Classification (LOAC hereinafter);

1. Data set narrowed in to contain data from Greater London region only

2. All variables were logarithmically transformed and range standardised within the region

3. The number of clusters in each group and their subdivision in lower tiers guided by distinct thresholds in the ratio of within and between cluster variability, in accordance with the Callinsky-Harabaz pseudo-F (Rabe-Hesketh \& Everitt, 2004).

Seven Supergroups were formed according to a distinct threshold in the information structure

(Figure 26). Each LOAC Supergroup was further subdivided into 49 Groups following the same procedure. This makes the LOAC Group tier comparable to the OAC Subgroup tier with 50 subgroups represented in Greater London (Table 18). 


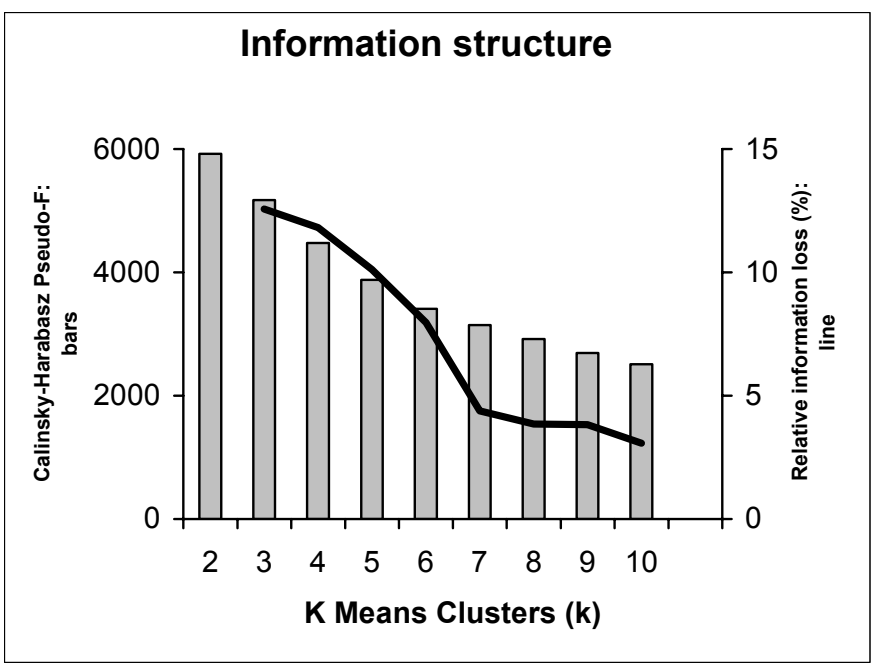

Figure 26 Stopping rule applied in the creation of London Output Area Classification (LOAC)

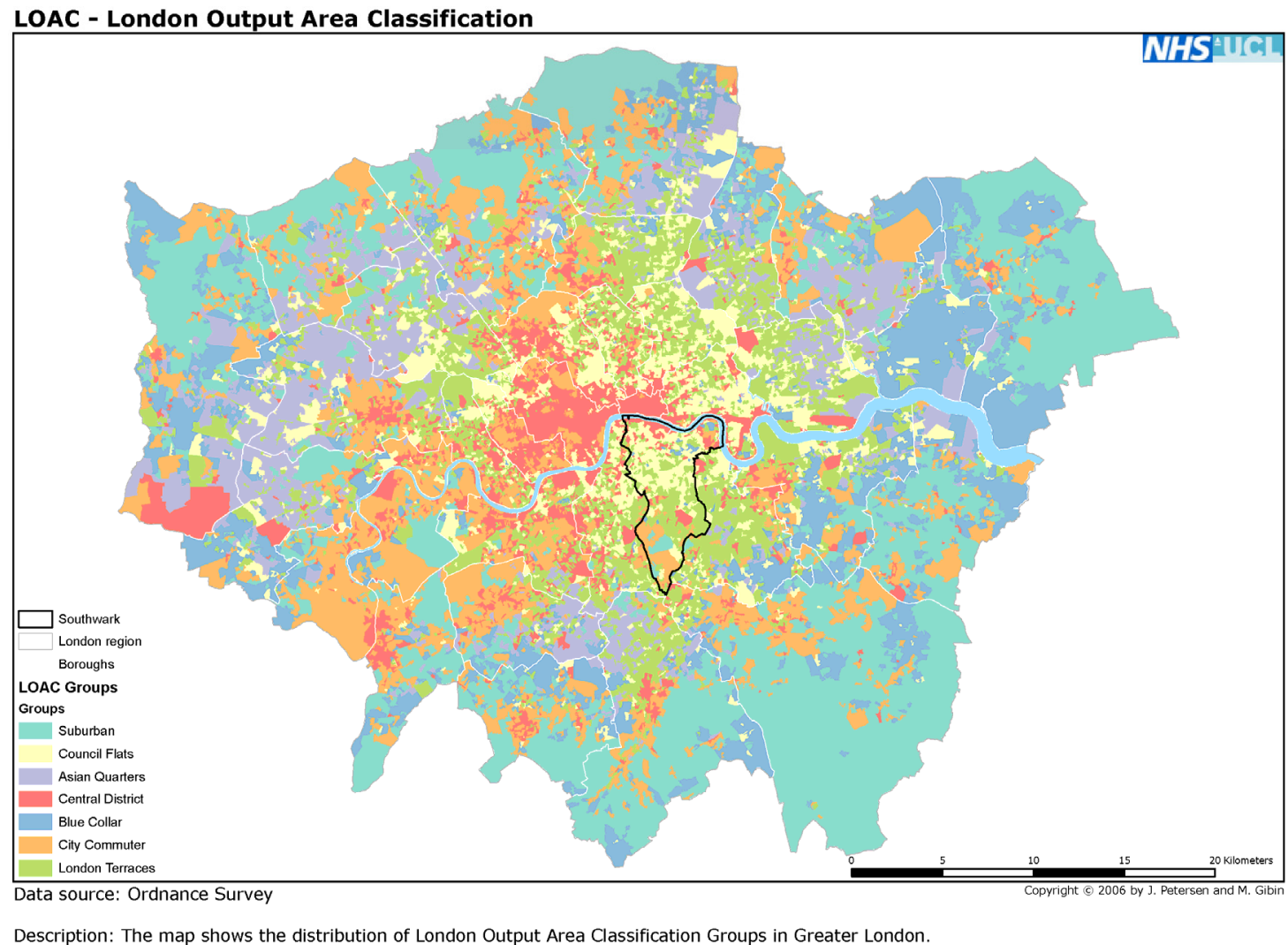

Figure 27 London Output Area Classification (LOAC Groups) 


\section{Geodemographics for neighbourhood targeting - a comparison}

To estimate the success of a geodemographic targeting strategy a hypothetical goal of reaching the top $20 \%$ of admissions was set for each disease and compared to reaching the same goal by a geographic targeting strategy. This follows the logic of a design used in medical diagnostics, i.e. comparing the overlap in frequencies of patients with a positive diagnosis using a high intervention test (the gold standard) with an alternative and usually cheaper low intervention test (Kirkwood \& Sterne, 2003). In this study geographic targeting was used as gold standard and geodemographic as the alternative test.

The admission data were aggregated at three geographical levels (unit postcode, output area and super output area) and labelled with the finest level codes of eight different geodemographic systems, e.g. the 50 subgroups in the Output Area Classification covering Greater London. Indices of Multiple Deprivation (IMD) was included as a potential segmentation system (50 quantiles according to IMD score in Greater London). As base population estimates Census 2001 headcounts by unit postcode were used and aggregated up to the different levels of geography according to the National Statistics Postcode Directory (Office for National Statistics, 2005).

Table 18 Geodemographic systems and Neighbourhood differention. NB only segments represented in Greater London are included; this e.g. excludes the Mosaic UK segment 'Rural isolation'.

\begin{tabular}{|c|c|c|c|}
\hline $\begin{array}{l}\text { Aggregation } \\
\text { level }\end{array}$ & $\begin{array}{l}\text { Neighbourhood } \\
\text { differentiation }\end{array}$ & $\begin{array}{l}\text { Number of segments } \\
\text { (Greater London) }\end{array}$ & Provider \\
\hline \multirow[t]{3}{*}{ Postcode } & Mosaic UK Type & 60 & Experian Ltd \\
\hline & Acorn Type & 58 & CACI \\
\hline & Health Acorn Type & 27 & CACI \\
\hline \multirow[t]{2}{*}{ Output Area } & OAC Subgroups & 50 & ONS \\
\hline & LOAC Groups & 49 & Internal \\
\hline \multirow[t]{2}{*}{$\begin{array}{l}\text { Super Output } \\
\text { Area }\end{array}$} & $\begin{array}{l}\text { Index of Multiple } \\
\text { Deprivation }\end{array}$ & 50 & $\begin{array}{c}\text { Department of } \\
\text { Communities and Local } \\
\text { Government }\end{array}$ \\
\hline & P2 Branches & 38 & Beacon-Dodsworth \\
\hline
\end{tabular}


The performance of the systems was evaluated by:

1) Gini coefficients weighted by Census 2001 population counts. The Gini coefficient is an overall mathematical measure of heterogeneity (or inequality). By weighting target frequencies with base population frequencies across all segments in a segmentation, it is intended to give higher values to a system that minimises base population to target ratio. The Gini coefficient is the industry standard for evaluating geodemographic systems (Callingham, 2006).

2) As an alternative two new performance indicators of targeting efficiency was created. They are both based on a ranking of geographic areas according to crude rate for each disease indicator. The areas containing the top $20 \%$ of all frequencies for a given disease were flagged as 'target'. These sets were treated as the diagnostic gold standard and the same procedure was repeated with area types within each geodemographic system in order to create the alternative diagnostic sets.

a. For each disease indicator and geodemographic system, diagnostic sensitivity was calculated as the percentage of gold standard admissions included in the alternative geodemographic target. Each system is hence evaluated against geographic targeting at the same level of aggregation, e.g. a unit postcode system such as Mosaic UK is compared to a geographic targeting scheme ordering postcodes according to their crude disease rate and ditto with a system coded at Census output area level, etc.

b. The base population included in the geographic target of the top $20 \%$ of admissions (the gold standard) was divided by the number of admissions in the target for each disease to produce a numbers-to-target ratio. This was likewise repeated for the geodemographic target sets (the alternative standard). 
Age-standardising geodemographic profiles of health data

Because LOAC as a regional classification has more even-sized segments than any of the other geodemographic systems, including OAC, it enables age-standardisation of geodemographic risk profiles of e.g. hospital admissions. Age standardisation is dependent on detailed age-banded population estimates and many geodemographic systems are currently only delivered with total headcounts. LOAC is based on the Census geography and it is relatively simple to impute age-banded estimates for men and women from the Census itself or from the population estimates released by ONS annually at the lower layer super output area (LSOA, a LSOA typically contains 5 output areas) level. In this study ONS age banded population estimates for the same years as the HES extract (2001-2004) were used with the twist of assigning populations from the coarser lower layer super output area (LSOA) level data to the finer output area level (OA) proportionally to the population share of each OA in their respective LSOAs in the 2001 Census. ONS use different age bands for women and men in this data product: $0-15$ year, 16-29 year, 30-44 year for both sexes and 45-59 year and 60+ year for women, 45-64 year and $65+$ year for men. Age-specific population-years-at-risk denominators were obtained by summing the population estimates over the four years of observation. The age- standardisation of hospital admission ratios, SAR, followed Kirkwood and Sterne (2003) with $i$ as age band, $p_{i}$ as population-at-risk and $\lambda_{i}$ as the age-specific rate per population-at-risk;

$$
\begin{aligned}
S A R= & \frac{\text { Observed number of admissions }}{\text { Expected number of admissions }} \\
& \begin{array}{l}
\text { were the age specific rates the same } \\
\text { as in the reference population }
\end{array}
\end{aligned}
$$

The data were analysed with an internal reference, i.e. the age-banded population of Greater London as reference. The equivalent crude admission ratios, CAR, were calculated following the same formula as for SAR only with a net crude admission rate based on headcounts instead. To 
highlight the potential effect of age standardising the geodemographic risk profile crude and agestandardised ratios were compared in an attenuation index;

$$
\text { Attenuationindex }=\frac{S A R}{C A R} \times 100
$$

If the age-standardised ratio is lower than the crude ratio, the effect of residing in a given neighbourhood type is - all else equal - explained by a higher proportion of elderly in that population (also known as effect attenuation). The opposite case would be expected where crude rates are lower due to a lower proportion elderly - all else equal (also known as effect deattenuation).

\section{London Output Area Classification}

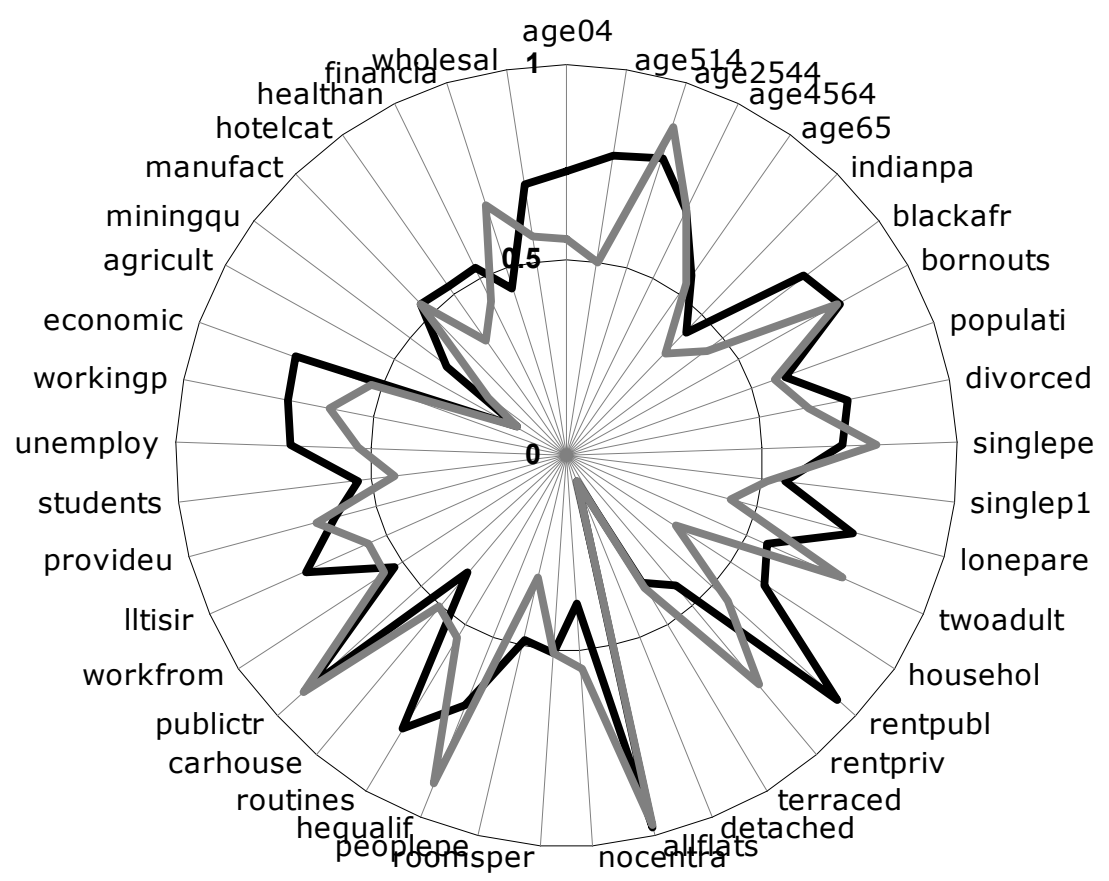

2 Publicly rented apartment blocks

4 Central District

Figure 28 Median Census attributes for two LOAC 1st tier clusters. Each attribute has been standardised to unity. For the full variable labels see Table 17. 


\section{Age structure}

London Output Area Classification

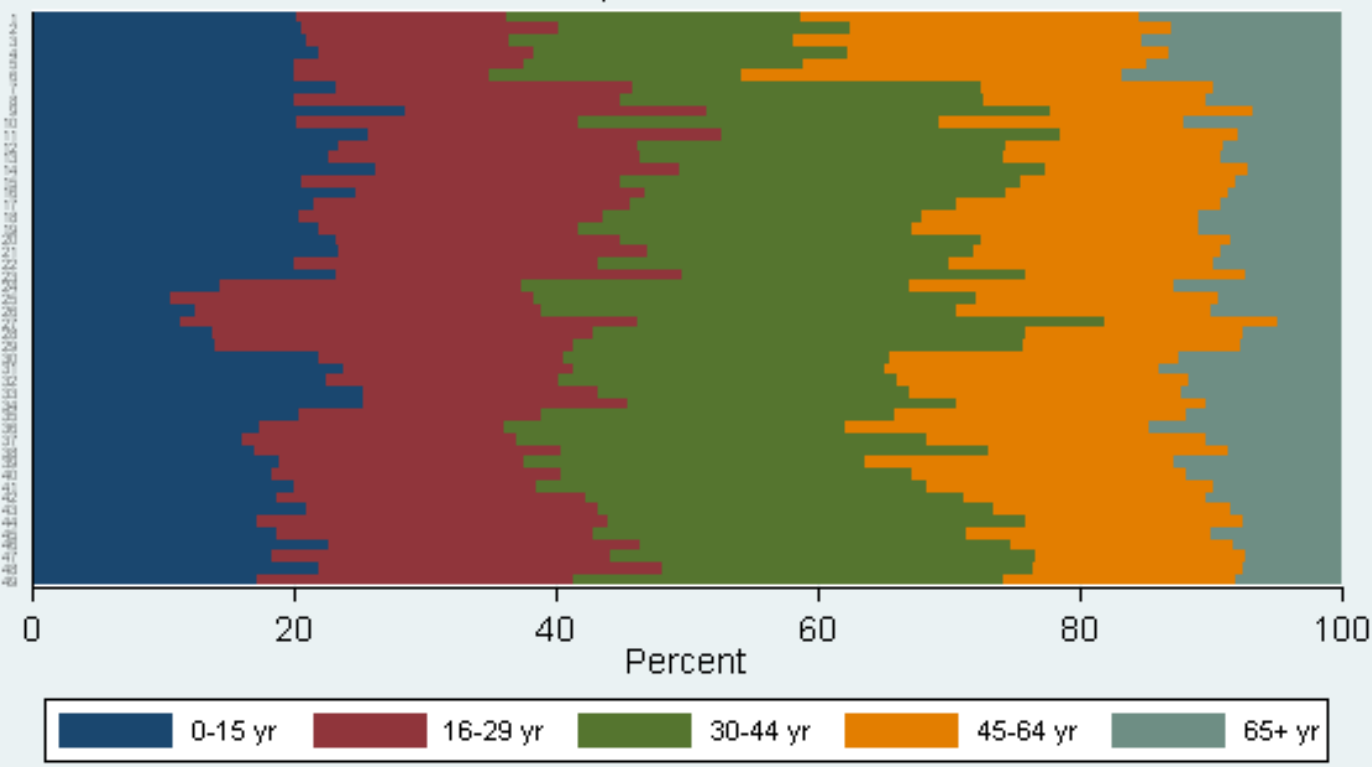

ONS male population estimates Greater London 2001-2004

\section{Age structure}

London Output Area Classification

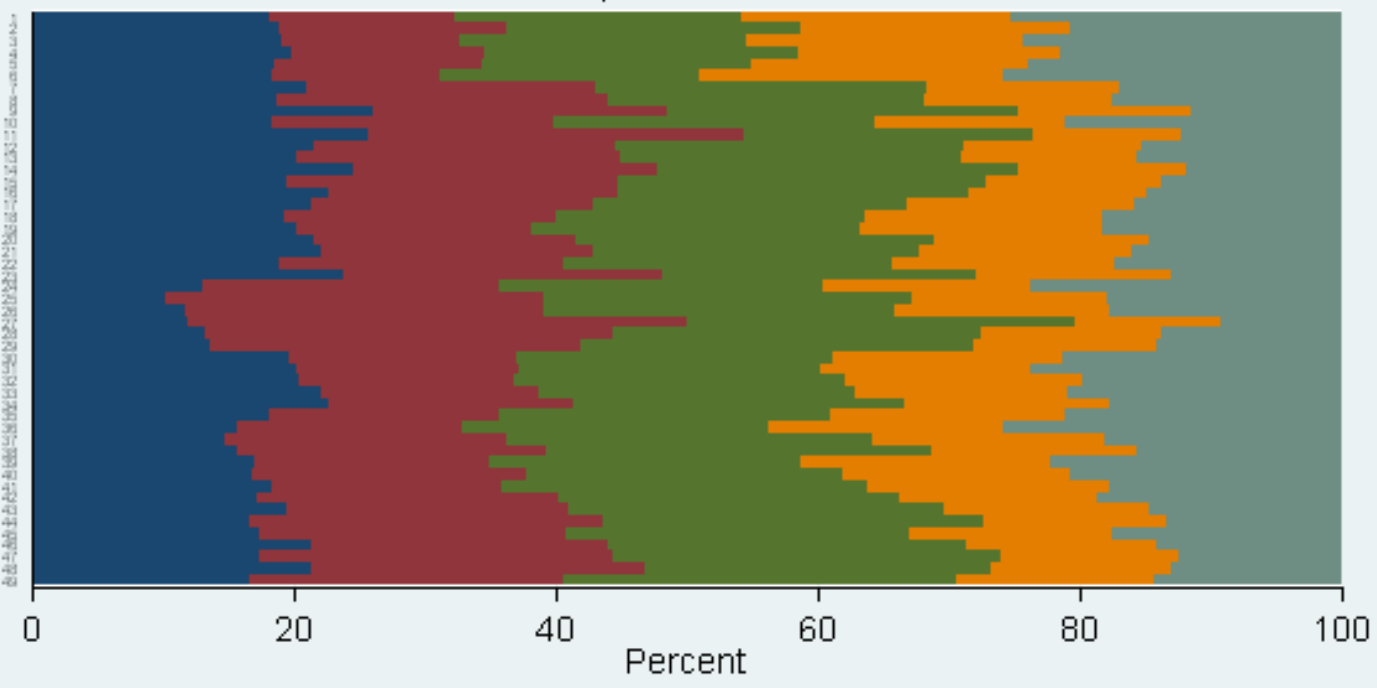

ONS female population estimates Greater London 2001-2004

Figure 29 Age structure in LOAC Groups. ONS population estimates 2001-2004. Top: Male population. Bottom: Female population. NB age bands differ between sexes 


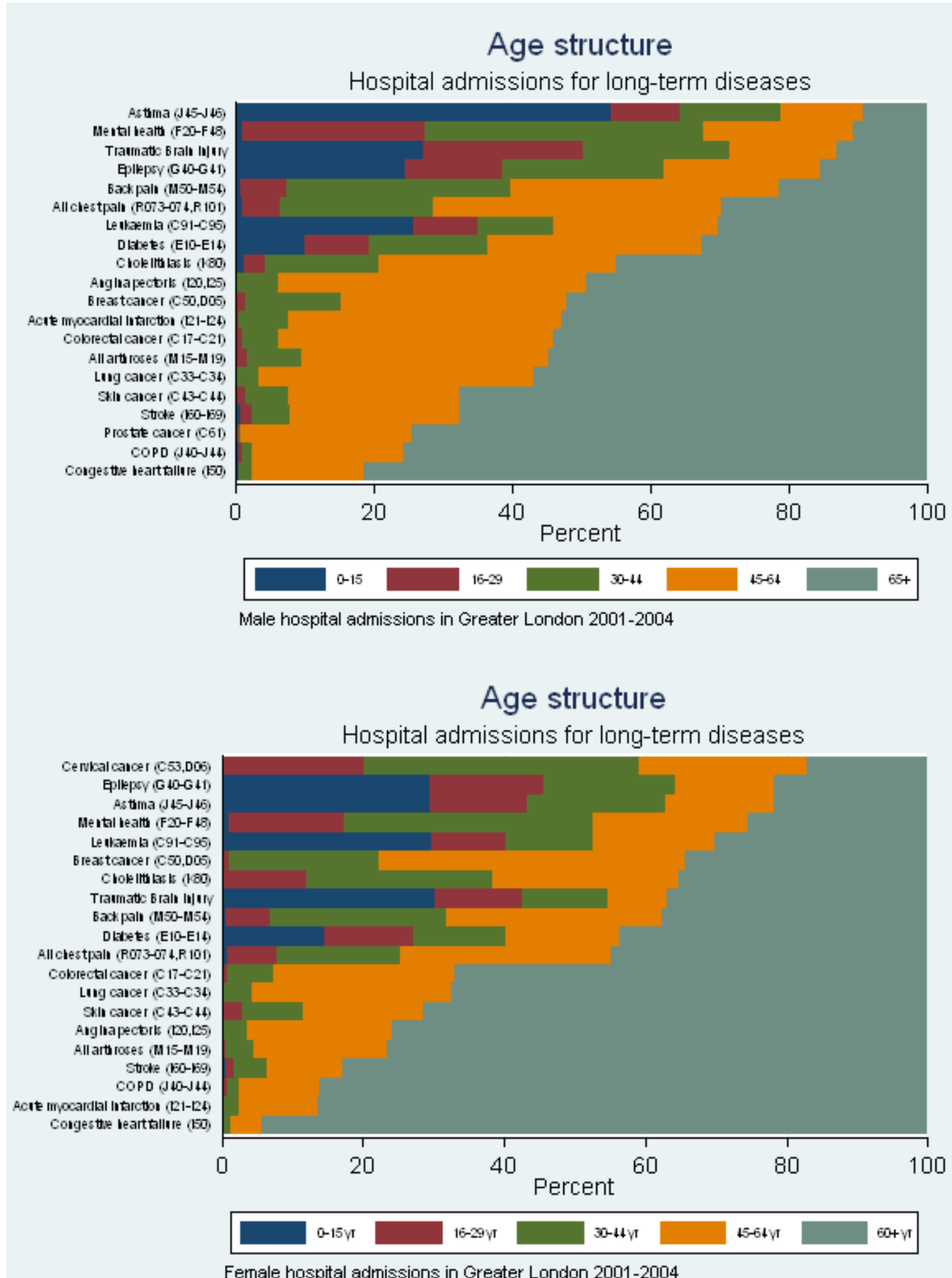

Fernale hospital admissions in Greater London 2001-2004

Figure 30 Age structure in hospital admissions. HES Greater London 2001-2004. Top: Male admissions. Bottom: Female admissions. NB age bands differ between sexes 


\subsection{Results}

The regional Census neighbourhood classification had more evenly sized segments that were better differentiated on distinctive regional characteristics subsumed within the broader brush national classifications. Inspection of the mapped results of the OAC versus the LOAC clearly reveals that the inner city neighbourhoods are better differentiated using the LOAC (Figure 25, Figure 27). In broad brush terms, this comparison revealed that the umbrella OAC "Multicultural" segment is replaced by three alternative LOAC segments, "2 Council Flats", "3 South Asian Quarters" and "7 London Terraces" (Figure 31). The main discriminatory variables were those related to tenure and ethnicity (Table 19). Comparing two LOAC Supergroups, "2 Council Flats" versus "4 Central District" across all clustering variables revealed marked differences in age structure, family structure, ethnic composition, education and occupational variables (Figure 28).

Using LOAC, patients in Southwark would be differentiated into neighbourhood groups with $52 \%$ of patients in the dominant group, " 2 Council Flats" in the first tier of divisions (Table 19) and only $14 \%$ in the second unnamed Group in the second tier. Within OAC the largest fragment occupied as already mentioned $86 \%$ in first tier ("Multicultural") (Table 14) and $80 \%$ in second tier. More importantly the regional classification avoided the fragment problem experienced with other systems (Table 14, Table 15).

The Gini coefficients showed the segmentations with two general commercial systems, Acorn and Mosaic, to be best optimised relative to the base populations (Figure 32). Comparable results was obtained with OAC (output area level); whilst the segmentation using the IMD performed the least well. Evaluating the systems relative to geographical targeting showed very low sensitivity overall. Postcode systems had the lowest sensitivity followed by output and super output area systems, respectively. The geodemographic strategies would in this study reach $20 \%$ of admissions, albeit not the same $20 \%$ as determined by the geographic targeting strategies. In fact 
the proportional overlap, i.e. the sensitivity, could be as low as $20 \%$ and never exceeded $50 \%$. Strategies using geodemographic systems at postcode level would potentially provide a cheaper means of reaching the target population because of the relatively low base populations indicated by the lower number-to-target indicator values.

The potential confounding of age and sex in geodemographic risk profiles of health outcomes were demonstrated in different ways. Detailed age-banded denominators were created using ONS population estimates for the same time period. The differences in age structure were clearly demonstrated across the different LOAC Groups, e.g. more elderly in suburban neighbourhood types (Figure 29). The age structure of patients of key long-term diseases was strongly but variedly associated with age. Congestive heart failure was, for instance, almost exclusively associated with the oldest age groups of women and men. Conversely, asthma predominantly affected children (Figure 30).

In campaigns that are more concerned with upstream causes age-standardisation may thus be an important addition to geodemographic profiling. Standard 'un-standardised' profiles of angina, for example, showed that the "1 Suburban" Supergroup neighbourhood types had crude admission ratios above the regional average $(\mathrm{CAR}=100)$, although when standardised for age they clearly fell below average $(\mathrm{SAR}=100)$ (Figure 33). The effect of a particular geodemographic neighbourhood type, as a container for lifestyle factors, was in this case confounded by a higher proportion of elderly; the geodemographic 'effect' was in other words attenuated by age.

Attenuation as well as de-attenuation effects were demonstrated across key long-term diseases for both women and men (Figure 34 and Figure 35). These effects were, as expected, strongest with diseases associated with old age in neighbourhood types with relatively many or, conversely, few elderly. 
Table 19 London Output Area Classification profile of patients registered with Southwark PCT, April 2006.

\begin{tabular}{|c|c|c|c|c|}
\hline LOAC Supergroup & Freq. & Percent & $\begin{array}{c}\text { Mean } \\
\text { IMD score }\end{array}$ & Typical Census attributes \\
\hline 1 Suburban & 3,346 & 1.1 & 16 & $\begin{array}{l}\text { Working age, White ethnic background, two- } \\
\text { adult households, large houses, higher education, } \\
2+\text { cars, routine jobs and part-time employment }\end{array}$ \\
\hline 2 Council Flats & 166,635 & 52.2 & 42 & $\begin{array}{l}\text { Children and young adults, Black ethnic } \\
\text { minorities and born abroad, divorced, single non- } \\
\text { pensioner households, lone parents, publicly } \\
\text { rented accommodation, apartment blocks, routine } \\
\text { jobs, longterm illnesses, unemployed, part-time } \\
\text { or economically inactive looking after family }\end{array}$ \\
\hline $\begin{array}{l}3 \text { South Asian } \\
\text { Quarters }\end{array}$ & 227 & 0.1 & 21 & $\begin{array}{l}\text { Families, South Asian communities and people } \\
\text { born abroad, two-adult households, terraced } \\
\text { housing, routine jobs and part-time employment }\end{array}$ \\
\hline 4 Central District & 28,297 & 8.9 & 25 & $\begin{array}{l}\text { Young adults, born abroad, singles and two-adult } \\
\text { households, renting privately, apartment blocks, } \\
\text { higher education }\end{array}$ \\
\hline 5 Blue Collar & 6,721 & 2.1 & 38 & $\begin{array}{l}\text { Families, White ethnic background, divorced, } \\
\text { lone parents, two-adult households, renting } \\
\text { publicly, terraced housing, routine jobs and part- } \\
\text { time employment, economically inactive looking } \\
\text { after family }\end{array}$ \\
\hline 6 City Commuter & 19,889 & 6.2 & 20 & $\begin{array}{l}\text { Working age, born abroad, single and two-adult } \\
\text { households, apartment blocks, terraced housing, } \\
\text { renting privately, large houses, higher education, } \\
2+\text { cars, part-time employment or economically } \\
\text { inactive looking after family }\end{array}$ \\
\hline 7 London Terraces & 94,136 & 29.5 & 33 & $\begin{array}{l}\text { Young adults, Black ethnic background and born } \\
\text { abroad, single and two-adult households, renting } \\
\text { publicly, apartment blocks, terraced housing, } \\
\text { higher education, routine jobs, longterm } \\
\text { illnesses, part-time employment or economically } \\
\text { inactive looking after family }\end{array}$ \\
\hline Total & 319,251 & 100.0 & 36 & \\
\hline
\end{tabular}



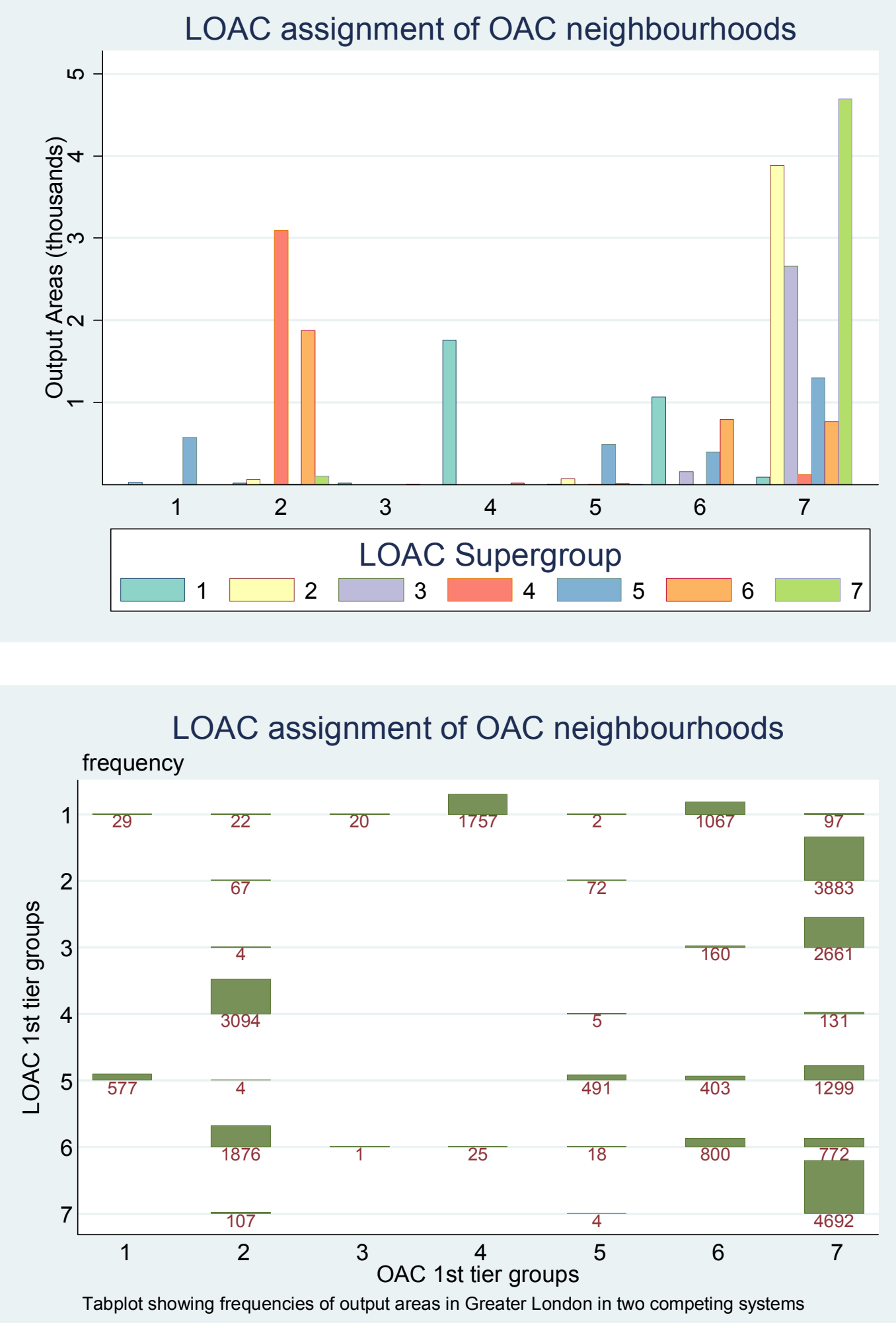

Figure 31 LOAC to OAC assignment of neighbourhoods 

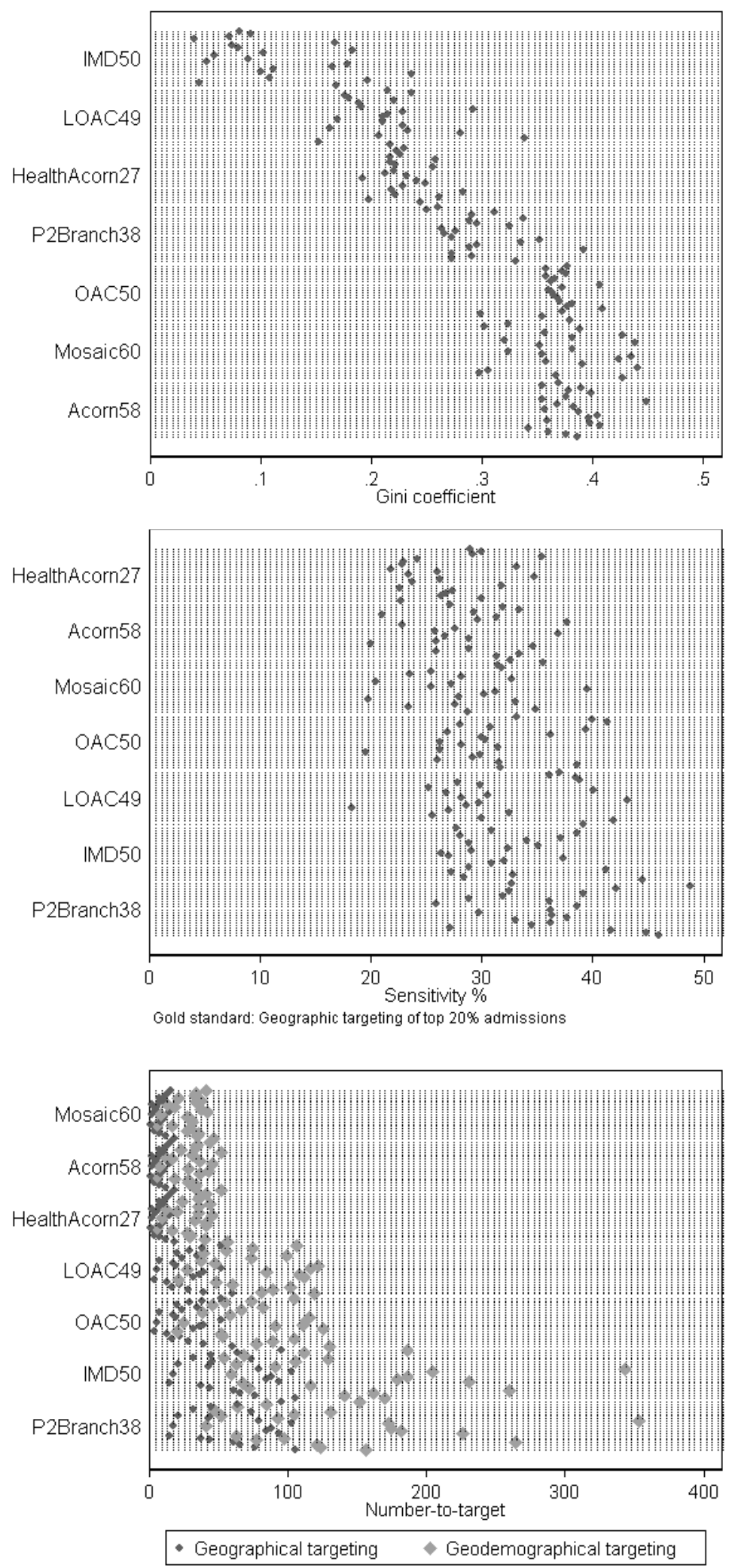

Figure 32 Top: Gini coefficients for chronic diseases across seven different geodemographic systems in horizontal panels. Middle: Sensitivity relative to geographic targeting as gold standard. Bottom: Number-to-target of same. 

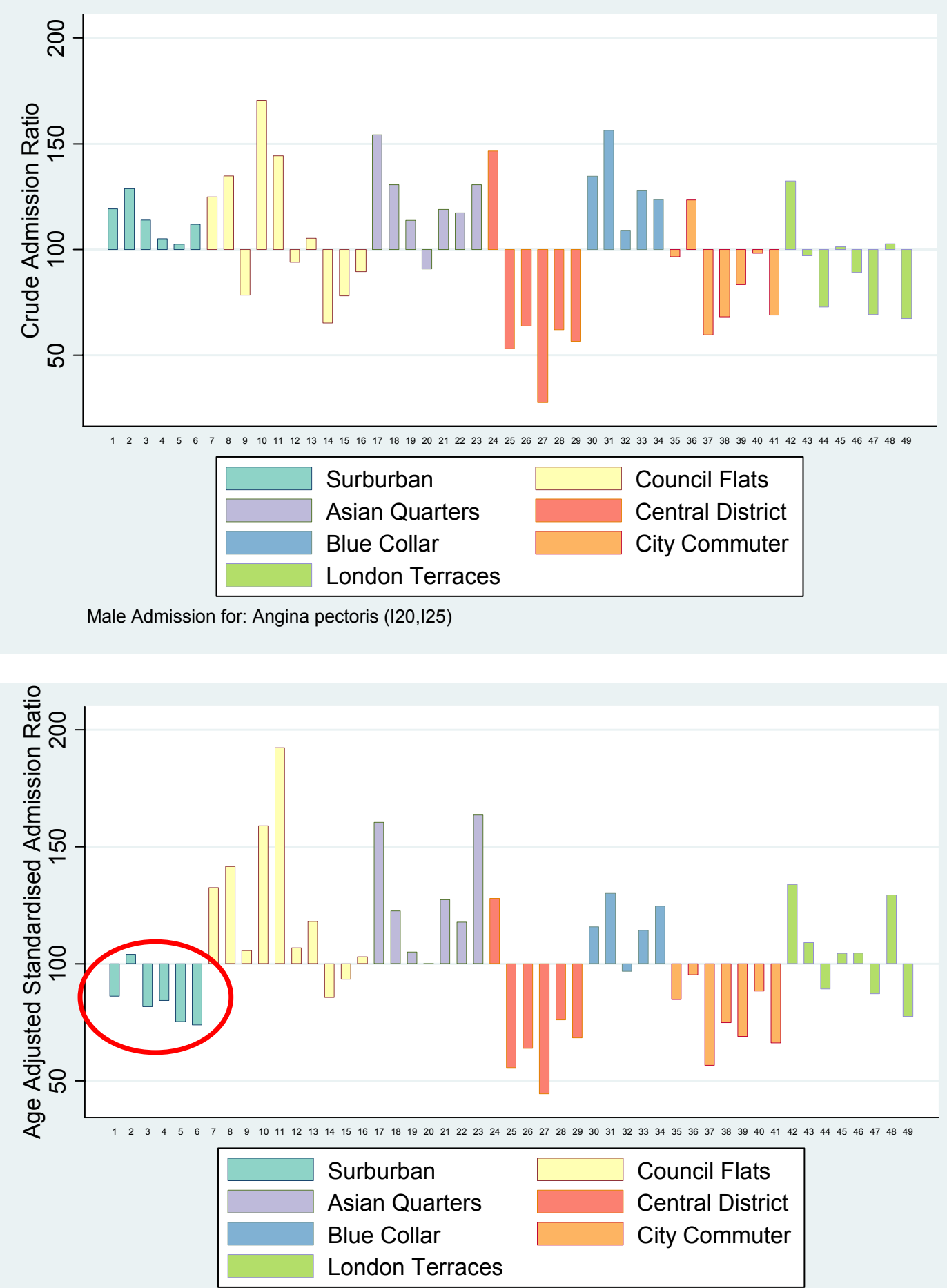

Male Admission for: Angina pectoris $(120,125)$

Figure 33 Geodemographic profile (LOAC) for Angina pectoris hospital admissions. Top: Crude admission ratios. Bottom: Age-standardised ratios. NB Shift in the Suburban category with age standardisation 


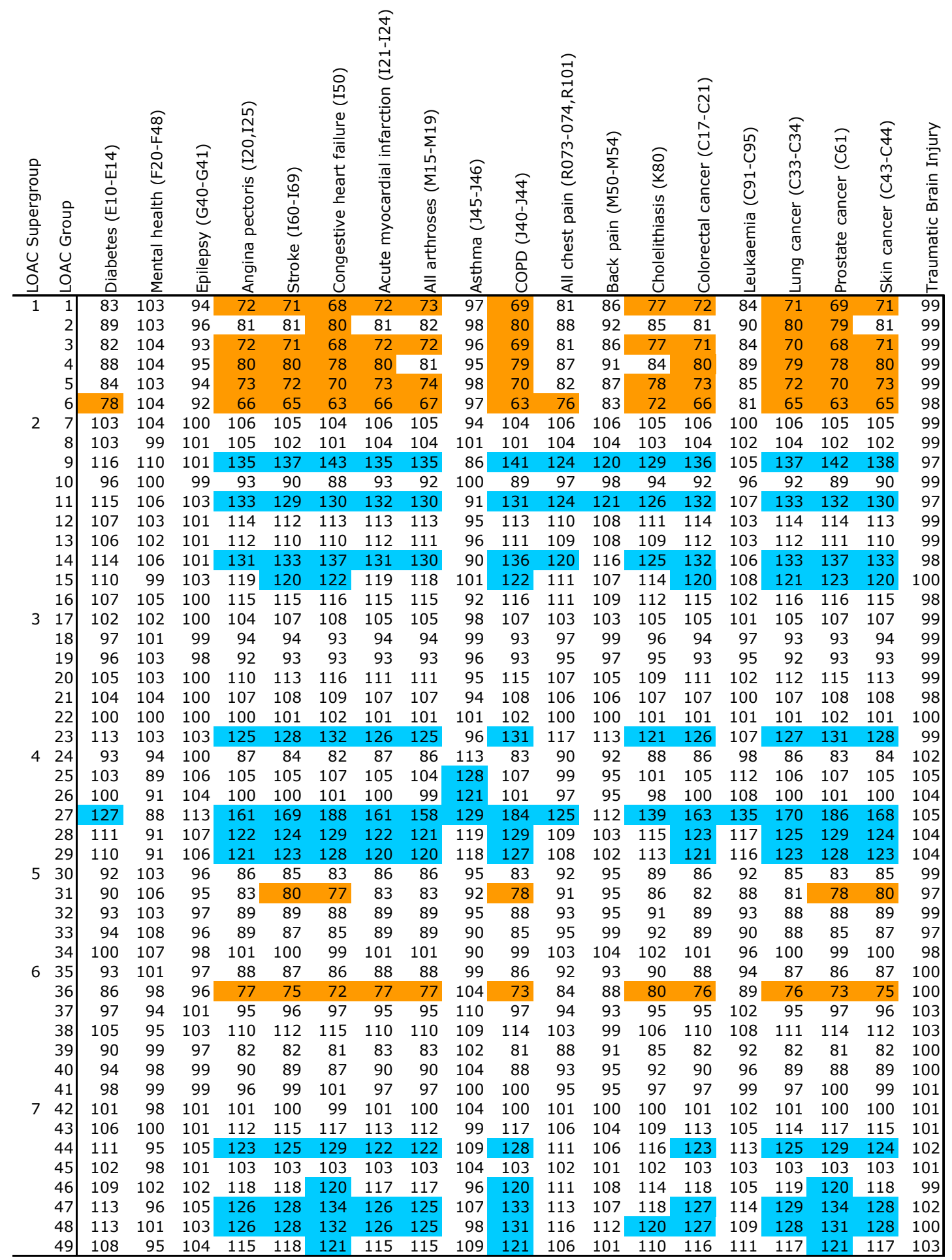

79 Attenuation - Age-standardisation decreases admission ratio

121 Deattenuation - Age-standardisation increases admission ratio

Figure 34 Effect of age standardisation of hospital admission ratios for key long-term diseases. Male patients in Greater London 2001-2004. Shading: Effects 20 percent below (orange) or above the crude ratios (blue). 


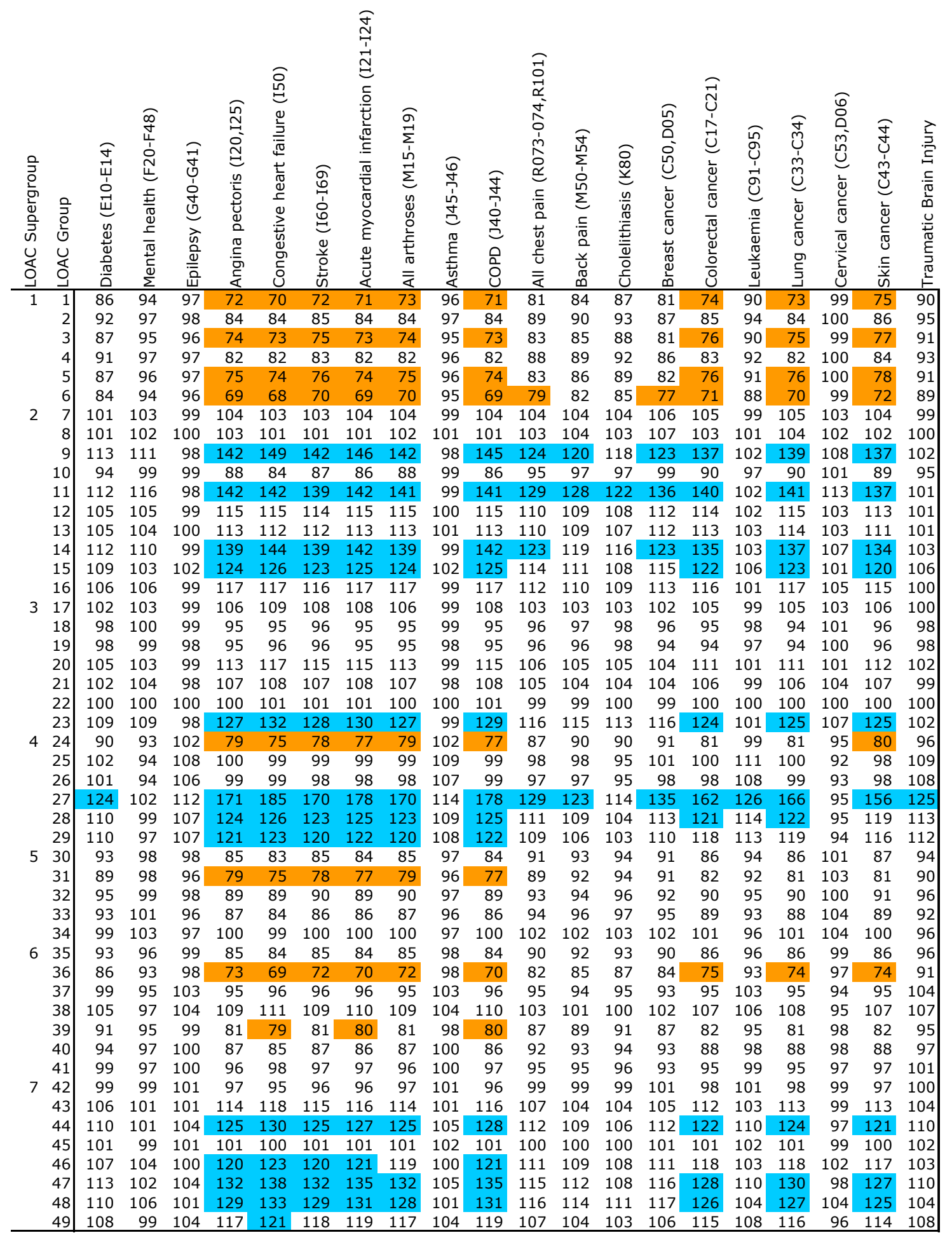

79 Attenuation - Age-standardisation decreases admission ratio

121 Deattenuation - Age-standardisation increases admission ratio

Figure 35 Effect of age standardisation of hospital admission ratios for key long-term diseases. Fe60male patients in Greater London 2001-2004. Shading: Effects 20 percent below (orange) or above the crude ratios (blue). 


\subsection{Discussion}

\section{Regional geodemographics}

A regional geodemographic system would address the regionality and fragment problems experienced when mounting targeted campaigns in a local context using national systems. As an approach however it does not address the problem that many critical health behaviours are studied in surveys using a national sampling frame. Health Survey for England, for instance, conducted 21,000 interviews in 2006 based on a national sampling frame stratified geographically to represent each of the 28 Strategic Health Authorities in England. This number of interviews would be largely insufficient for geodemographic profiling in a regional system. Perhaps decision makers in local authorities and public health institutions in the future will consider the value of different geodemographic systems: national systems for data sources with a national sampling frame and regional systems for data with a regional sampling frame.

\section{Targeting efficiency}

Besley and Kanbur (1990) proposed that given scarce resources geographical targeting should favour areas in order of need until the available budget is exhausted. Any targeted strategy based on aggregated data however opens up issues of inclusion and exclusion. A public health campaign strategy, for example, may include individuals who are not at risk for the health outcome it was designed to counter or ameliorate; i.e. the problem of inclusion. Conversely there may be citizens with those exact needs that are excluded by the strategy simply by having the 'wrong' postcode; i.e. the exclusion problem. Both these problems pertain to efficiency and fairness considerations of a strategy and should be evaluated in line with other welfare policy intervention (see section on utilitarianism in part one).

The empirical analysis presented here suggested that all of the geodemographic systems had a low sensitivity in comparison with geographic targeting. This exemplifies exclusion problems: 
geodemographic allocation strategies would still reach $20 \%$ of admissions, albeit not the same $20 \%$ displaying the highest needs as determined by geographic targeting. The relatively high number-to-target ratios demonstrated the inclusion problems in both types of targeting; although geodemographic strategies would be more expensive to deploy, i.e. in terms of mail shots or other campaign means magnified by base population numbers.

The results of the diagnostic approach deployed here also suggest that, for these health indicators, it is the geographic order of aggregation (unit postcode, output area, or super output area), more than the geodemographic classifications themselves, that is critical for the accuracy of targeting. This also questions whether Gini coefficients, however popular, are in fact too sensitive to the huge within-region variability in base population sizes and applied in this way hence become a measure of population size heterogeneity rather than actual targeting 'efficiency'.

In evaluating geodemographic systems for the targeting of public health campaigns we need to consider two different situations. First, cases where data on actual demands are at hand and geographic targeting would thus be more accurate, fairer and potentially less costly for campaign use than a geodemographic alternative. Second, cases where we would like to predict lifestyle information. In these cases geodemographic systems have potential value. Choosing a geodemographic system will rely on a number of factors, including budget and health data quality. This study suggests that postcode systems are not necessarily more accurate, but that they would be cheaper to deploy in campaigns; the classic dilemma of balancing equity and efficiency in other words.

\section{Age-standardisation}

In a modern society, safe from immediate dangers, lurks the risk of long-term diseases that creeps up on the unsuspecting partly due to the fact many of us live a lot longer - life expectancy has noticeably doubled over the last two centuries (Haines, 1999). Another component to the rising trend of long-term diseases is associated with the diversity of lifestyles. Properly 
constructed epidemiological studies of these health outcomes are needed, but are at the same time costly, prone to selection bias and in many instances will require longitudinal studies following a large number of individuals for several decades. Could geodemographic neighbourhood classifications, in an epidemiological sense, be used as a composite exposure variable that would explain differential health outcomes? Geodemographic neighbourhood types are, if not manifestations, then representations of the societal tapestry of social, socio-economic, demographic, cultural and other lifestyle factors as it unfolds in real-time (Figure 36).

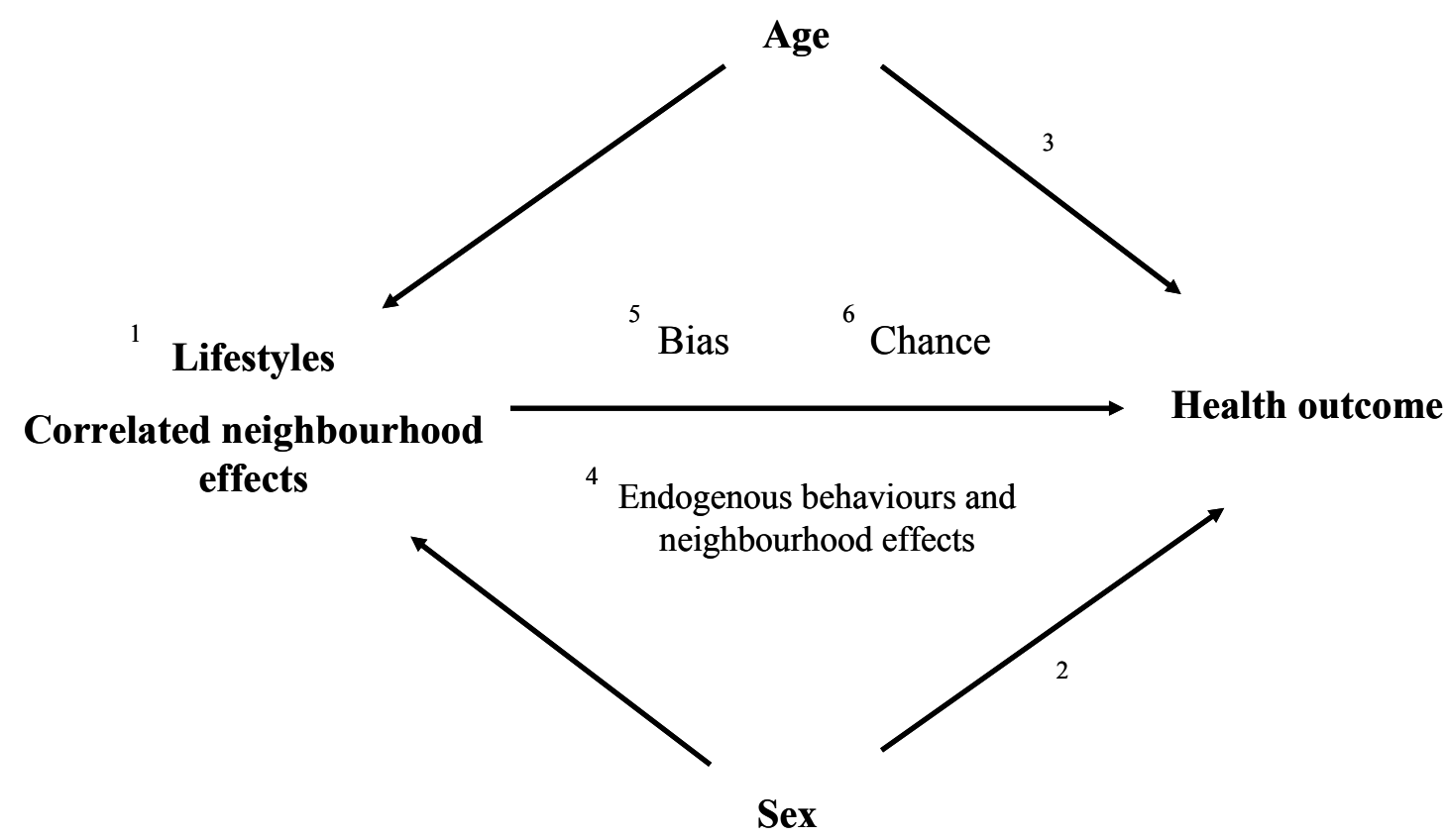

Figure 36 The geodemographic 'pathway' to health outcomes (see text for annotations)

The geodemographic 'pathway' to health (Figure $36(1))$ is however fraught with difficulties and circularities. No areal aggregation will ever be a stable and easily labelled container of exposures. Ideally geodemographic clusters would - if on a coarse scale - reflect patterns of different capabilities, behaviours and choices (chapter 1). Biases (Figure 36 (5)), especially selfselection and choosing neighbourhoods, have to be acknowledged on this 'pathway' too whether 
they condition or in actual fact obscure other causal factors (chapter 7). While some behaviours are likely to be 'part of the packaging' or - in other words - correlated with other factors defining geodemographic clusters, others may inherently be of a more local nature (Figure 36 (4), see also discussion on endogenous behaviours in chapter 3 or chapter 6). The examples in this chapter confirmed the power of two personal data available in most secondary data sets on health, i.e. sex (Figure 36 (2)) and age (Figure $36(3)$ ). Sex was as a factor treated separately in the analyses of age-standardisations for conceptual and practical reasons. First, some exposures are different for men and women even if they share household, e.g. occupational exposures. Second, the biology and aetiology of diseases are in some cases different. Third, women on average live longer and are - all else equal - more likely to encounter the diseases associated with old age. Finally, a practical constraint was in this case that the denominator data from ONS were released with age bands that follow the pension age (still different for women and men at the time of writing). Age was also confirmed as an important confounding factor for geodemographic profiles of hospital demand especially for diseases more strongly associated with old age, e.g. angina, due to the variation in age structure in different neighbourhood types (Figure 29, Figure 30, Figure 33). Age-standardisation would be a simple yet powerful addition to geodemographic profiles, where the primary concern is 'upstream' factors. The next chapter will take a different approach and consider geodemograhics as a way of segmenting the de facto market for hospital demand.

\subsection{Conclusion}

The regional geodemographic system seems a promising addition to the geodemographic 'family' of planning and social research tools. The prime advantage of geodemographic systems is that the segments as representations are relatively homogenous in socio-economic terms and thus becomes the mirror distortion of different lifestyles (Webber \& Longley, 2003). A regional classification will in this sense always be more relevant to a local context. With regards to the health domain many surveys are nonetheless based on a national sampling frame and hence will 
continue to need national classifications for their interpretation. In time decision makers may come to appreciate the value of different geodemographic classifications for different purposes; some national and some regional or local.

Geodemographic classifications are useful for differentiating between neighbourhoods with different demands and needs. The geodemographic targeting of public health campaigns may, however, not necessarily always be the most efficient compared to alternative means of geographical targeting. The inherent problems of inclusion and exclusion apply to this type of targeting as well as in any other strategy based on aggregated data (Besley \& Kanbur, 1990). There seems no immediate solutions to this problem. Two geodemographic systems would not reach the same prediction for a local area simply due to the problems of reaching a standard methodology to generalise complex, multivariable data consistently. Different systems or zonations will invariably lead to different local estimations, the Modifiable Area Unit Problem (Openshaw, 1984). As a consequence different areas and populations are selected and excluded in targeted campaigns. Two new performance indicators of targeting efficiency were proposed to quantify these problems: sensitivity (the degree of overlap with a geographic targeting alternative) and the number-to-target (the sum of target and non-target population included in a campaign relative to the target). The sensitivity of the different geodemographic systems proved, in an empirical example with geo-referenced hospital admission data, to be relatively monotonous within a given level of geographical aggregation. In this way there was only modest evidence of zone effects (the actual classification does not matter overall - in simple terms), whilst scale effects were clearly evident for the number-to-target criteria (scale matters!). In conclusion, the more fine scale geodemographic systems were superior, not in sensitivity which in general was lower, but by including a lower number of non-target population (people not eligible for the campaign) in their target. The number-to-target is thus of relevance for the evaluation of 
campaigns intensified by mailshots, interviews or other methods involving direct contact to either households or individuals.

Geodemographic profiles of health outcomes revealed stark differences in the apparent health needs of different populations. Geodemographics can in this way be said to re-describe the health inequality problems exposed by area deprivation scores or social class measures. Compared to the latter geodemographics however presents a richer tapestry of potential factors that at least hypothetically can explain emerging health problems. Labels such as "social class IV" or "IMD score 27.9", on the other hand, seems less relevant to the interpretation of complex health information (Longley, 2005). Age standardisation of geodemographic profiles is rare; possibly because it requires age-banded denominator data and robust estimates of risk across the different geodemographic segments, which again is hindered by small-number problems experienced when the current national geodemographic systems are applied locally, i.e. the fragment problem. The empirical example with geodemographic profiles of hospital admission data demonstrated that age-banded populations-at-risk denominators were achievable with the current official population estimates. It was furthermore demonstrated that robust estimates of hospital admission risk were achievable when using a new, regional geodemographic system with more evenly sized base populations. The value of age-standardisation was evident especially from geodemographic profiles of long-term diseases associated with elderly patients. Elderly with poorer health would for instance be 'hidden' if residing in areas with a younger-than-average age structure. Suburban neighbourhoods would in the same example 'qualify' for targeting, although the implied association with 'upstream' policy variables would be confounded by differences in the base population age structure alone.

The power of geodemographics may, however, not be 'a line of attack to disentangle causal factors in health inequality studies', but more simply a way to uncover interesting patterns of demand, which is the topic of the next chapter. 


\section{Chapter 9 Neighbourhood classification based on data of hospital}

\section{demand}

The National Health Service data infrastructure has improved considerably in the last few

years. This will open up for new opportunities to integrate, link and analyse secondary data more efficiently and at the same time deliver information to local services in an automated fashion. Healthcare planning is in other words experiencing an information explosion. With this of course comes the challenge of making new data useful - to avoid a concomitant understanding implosion.

Geodemographics is, as demonstrated in other parts of this thesis (chapter 7 and 8), a useful data mining tool to describe problems of health inequality. In chapter 8 the value of geodemographics was also considered as a proxy for a host of lifestyle factors of importance for health outcomes. It was demonstrated that by standardising profiles by age and sex would be valuable when designing public health campaigns focused on potential upstream factors. This chapter will take a different approach. Traditionally, geodemographic systems have been used by commercial companies to map out demand for their products. For many planning purposes in the public sector this approach may similarly be justified. Many healthcare demands are overlapping and these composite healthcare needs may nonetheless be lost in the profusion of new data, indicators and indices available to planning authorities (see Figure 37 for an example of the diversity of hospital demands for just a few long-term diseases). The aim with this chapter is to develop a bespoke geodemographic system solely based on hospital demand data and discuss the potential benefits of this alternative approach. 

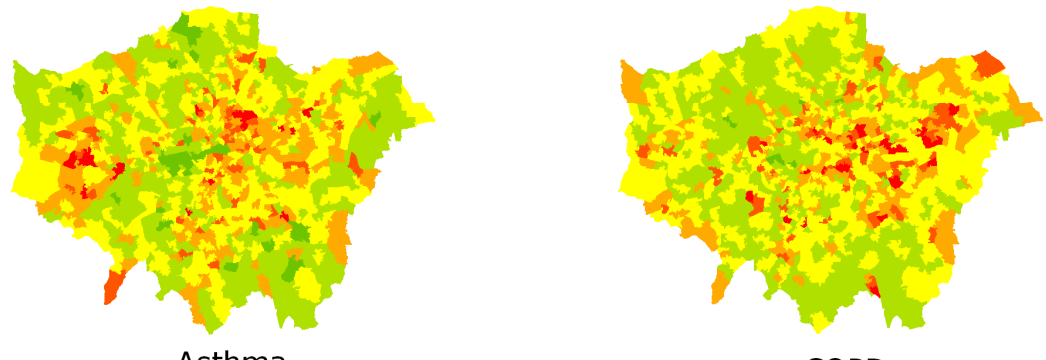

COPD
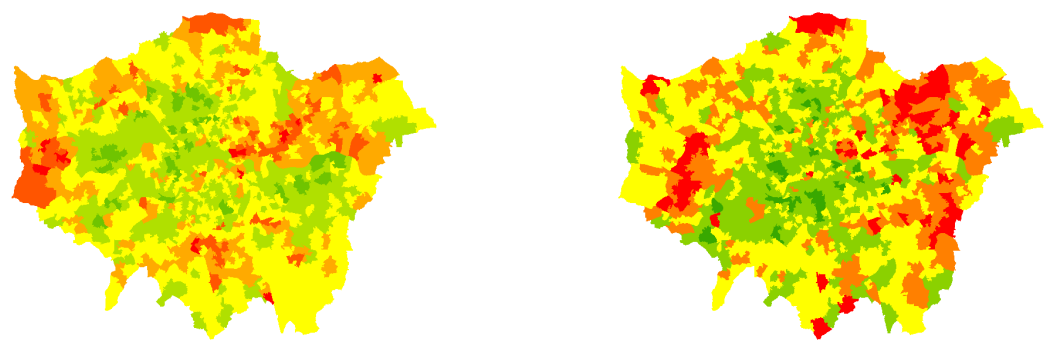

Chest Pain

Angina
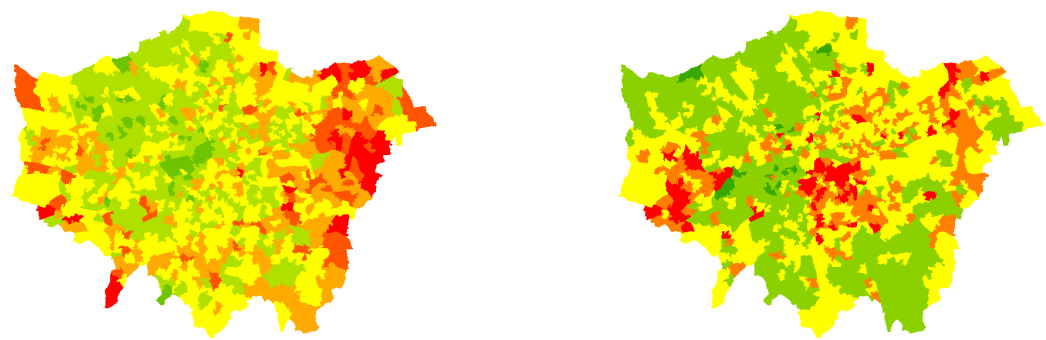

Gall Stones

Diabetes

Standard deviations from mean

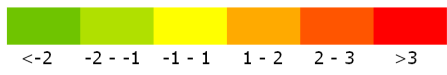

Figure 37 Geographical distribution of hospital demand of a six long-term conditions. Total admissions per population (middle layer super output area level) classified according to standard deviation, Greater London 2001-2004 (inclusive) 


\subsection{Methods}

Postcode data for 21 of the most commonly occurring long-term diseases were obtained from Hospital Episode Statistics, 2001-2004 (see section 8.3). The data were aggregated to middle layer super output area (MSOA) level and denominators were appended from ONS population estimates from the same years as a total population-years-at-risk. Crude rates of total hospital demand (admissions per million person-years-at-risk) were created and inspected visually for deviation from the normal distribution (Figure 38). Generally many of the diseases are rightskewed and the variables were subsequently logarithmically transformed and range standardised to reduce the potential impact of outliers on the stability of the clustering. Two variables, leukaemia and cervical cancer, were particular skewed and were excluded from the clustering process, but kept for post hoc description of clusters.

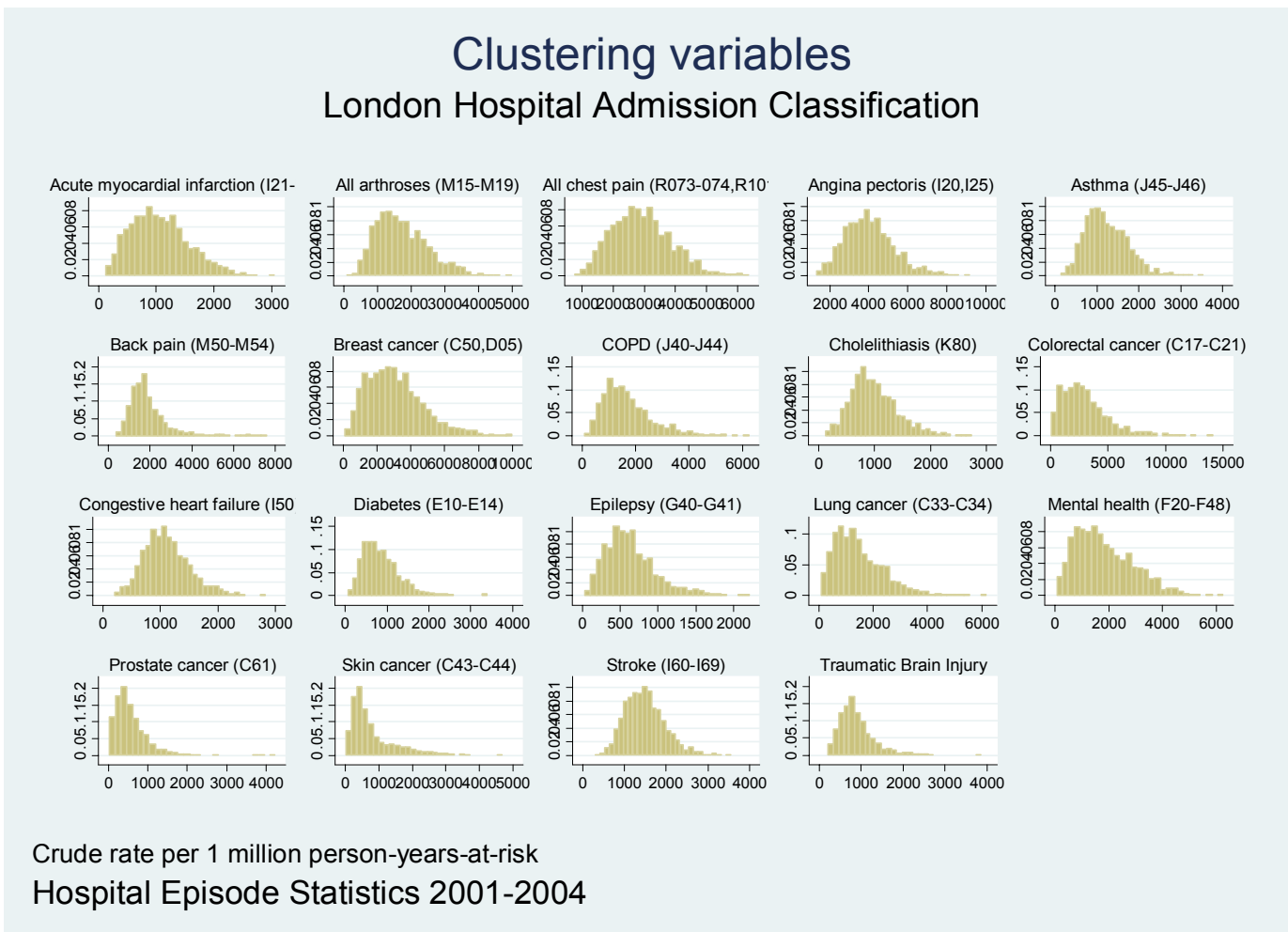

Figure 38 Distribution of clustering variables in London Hospital Admission Classification. Y-axes show bin class fraction. $\mathrm{X}$-axes crude per million person-years-at-risk (prior to log transformation) 
The variables were also inspected visually for any specific problems of collinearity. Clustering results are usually less stable if clustering variables are poorly sampled, naturally skewed or strongly correlated with other variables (Rabe-Hesketh \& Everitt, 2004).

The variables where clustered in Stata 9 (College Station, USA) using the k-means clustering algorithm with Euclidean distance as the cluster similarity measure. To assess whether there were naturally forming clusters, the number of clusters, $\mathrm{k}$, was increased iteratively and a ratio of intra and inter-specific cluster variation (Callinsky-Harabaz Pseudo F) was obtained and plotted against k (Figure 39) (Rabe-Hesketh \& Everitt, 2004). There were no abrupt changes in the curve and seven clusters were selected as the number nearest to a $50 \%$ reduction of the information statistics from the first clustering $(\mathrm{k}=2)$ (Kaufman \& Rousseeuw, 2005).

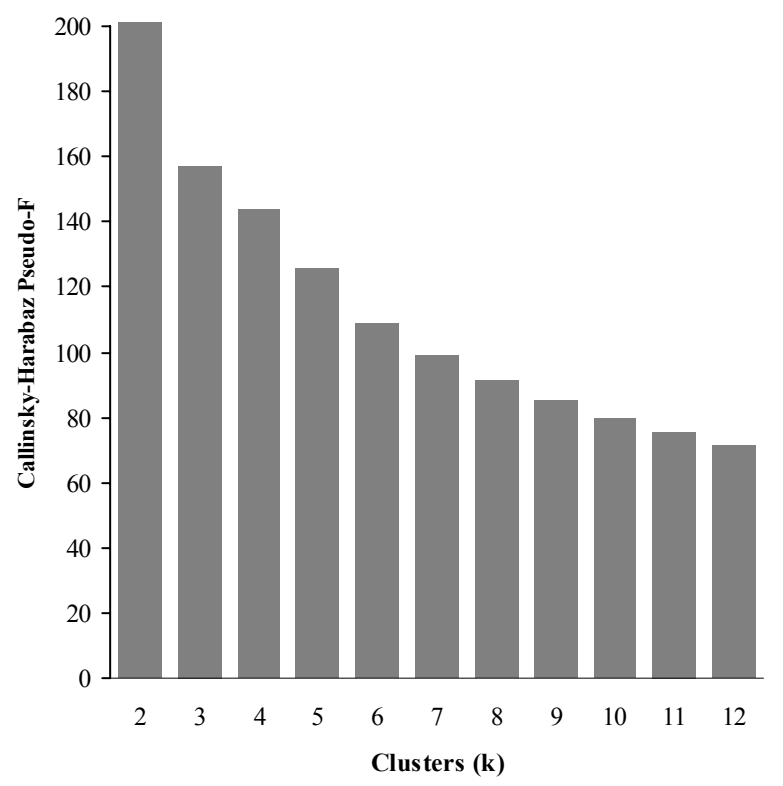

Figure 39 Information loss curve for London Hospital Admission Classification. Seven clusters were used $(k=7)$.

The median standardised values were plotted for all segments across all variables in a radial plot to highlight the characteristics of the different clusters in this new bespoke classification, 
London Hospital Admission Classification (LHAC) (Figure 40). In many cases geodemographic systems are furnished with propensity indices for various behaviours and not prevalence rates due to the fact that the data sources are not comprehensive. In this case Hospital Episode Statistics is assumed to be comprehensive and prevalence rates were thus calculated as total admissions per million person-years-at-risk (Table 20).

Table 20 Median crude hospital admission rates across London Hospital Admission Classification (per 1 million person-years-at-risk). Shading indicates above median rates

\begin{tabular}{|c|c|c|c|c|c|c|c|c|c|}
\hline \multirow[t]{2}{*}{ Label } & \multirow[t]{2}{*}{ Speciality } & \multirow[t]{2}{*}{ Chronic disease indicators } & \multicolumn{7}{|c|}{ London Hospital Admission Classification } \\
\hline & & & 1 & 2 & 3 & 4 & 5 & 6 & 7 \\
\hline diabetes & Endocrinology & Diabetes (E10-E14) & 522 & 1087 & 992 & 896 & 1104 & 587 & 668 \\
\hline mentalhe & Mental health & Mental health (F20-F48) & 1448 & 2685 & 1770 & 2321 & 1747 & 795 & 1385 \\
\hline epilepsy & Neurology & Epilepsy (G40-G41) & 406 & 921 & 723 & 639 & 695 & 499 & 492 \\
\hline angina & Circulatory & Angina pectoris $(\mathrm{I} 20, \mathrm{I} 25)$ & 2771 & 4018 & 4921 & 2722 & 5498 & 4184 & 4049 \\
\hline stroke & & Stroke (I60-I69) & 1048 & 1554 & 1537 & 1191 & 1970 & 1181 & 1567 \\
\hline heartfai & & Congestive heart failure (I50) & 675 & 1125 & 1284 & 848 & 1523 & 1057 & 1109 \\
\hline heartatt & & Acute myocardial infarction (I21-I24) & 527 & 848 & 1305 & 590 & 1648 & 1108 & 1275 \\
\hline arthriti & Musculoskeletal & All arthroses (M15-M19) & 1033 & 1354 & 2075 & 1159 & 2551 & 1875 & 2004 \\
\hline asthma & Respiratory & Asthma (J45-J46) & 711 & 1636 & 1518 & 1275 & 1354 & 1002 & 962 \\
\hline copd & & COPD (J40-J44) & 818 & 2337 & 1727 & 1507 & 2490 & 1038 & 1266 \\
\hline chestpai & Pain symptoms & All chest pain (R073-074,R101) & 1752 & 3178 & 3845 & 2269 & 3470 & 3117 & 2682 \\
\hline backpain & & Back pain (M50-M54) & 1255 & 2012 & 2737 & 1583 & 2198 & 1518 & 1743 \\
\hline gallston & Digestive & Cholelithiasis (K80) & 549 & 1006 & 1131 & 803 & 1450 & 647 & 1012 \\
\hline breastca & Cancers & Breast cancer (C50,D05) & 3217 & 2864 & 1699 & 3006 & 3985 & 1608 & 3835 \\
\hline bowelcan & & Colorectal cancer $(\mathrm{C} 17-\mathrm{C} 21)$ & 2896 & 2606 & 1651 & 2351 & 4616 & 1159 & 3915 \\
\hline leukaemi & & Leukaemia (C91-C95)* & 1416 & 1575 & 1956 & 1512 & 2302 & 1201 & 1768 \\
\hline lungcanc & & Lung cancer (C33-C34) & 1053 & 1868 & 864 & 1286 & 2359 & 545 & 1489 \\
\hline cervcanc & & Cervical cancer $(\mathrm{C} 53, \mathrm{D} 06)^{*}$ & 268 & 367 & 372 & 345 & 442 & 285 & 400 \\
\hline prostcan & & Prostate cancer $(\mathrm{C} 61)$ & 533 & 470 & 453 & 476 & 765 & 413 & 701 \\
\hline skincanc & & Skin cancer (C43-C44) & 524 & 461 & 815 & 480 & 1378 & 698 & 1072 \\
\hline tbi & Other & Traumatic Brain Injury & 683 & 1317 & 821 & 1067 & 866 & 508 & 676 \\
\hline & & Mean IMD score & 20 & 38 & 26 & 32 & 24 & 16 & 15 \\
\hline
\end{tabular}




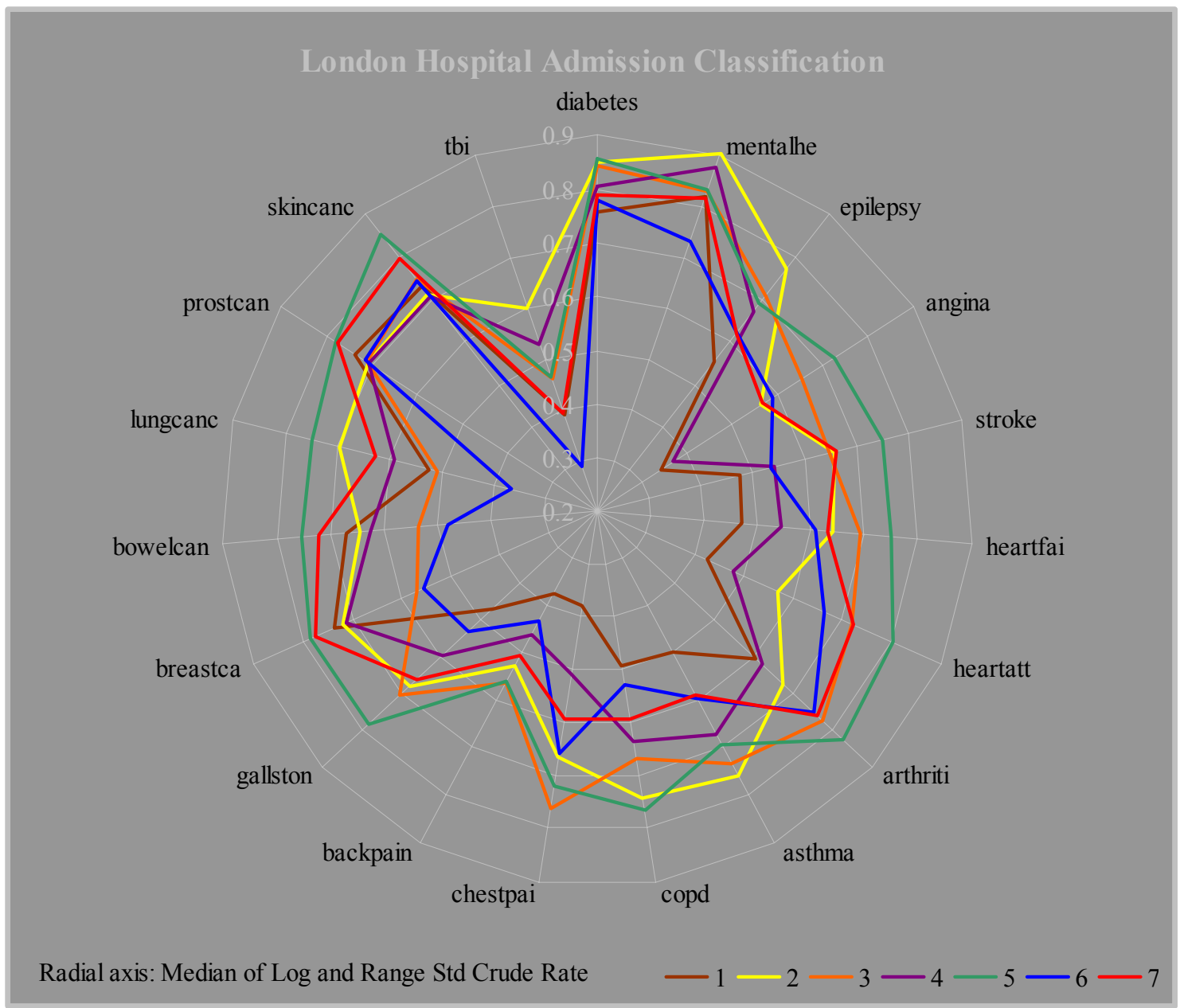

Figure 40 London Hospital Admission Classification. Median of standardised attribute values for clustering variables For explanation to labels see Table 20.

The different neighbourhood types were characterised in a typology based on above median admission rates (Table 20, Figure 41). Mapping LHAC with LOAC showed where the two classifications overlapped most clearly, i.e. between LHAC "5 All care intensive diseases" and LOAC "5 Blue Collar" (Figure 27, Figure 41). Interestingly some of the more affluent areas (based on IMD scores) score had high admission rates of certain cancers, LHAC "2 Breast, Bowel and Prostate cancer", or angina, "6 Angina". Diseases such as mental health and traumatic brain injury were more associated with inner city areas ("4 Mental health, traumatic brain 
injury"), while skin cancer seems more common in suburban segments, presumably because of the higher proportion of elderly, white patients.

\subsection{Discussion}

Health geodemographics was introduced in chapter 7 as a methodology for the integration of data and information on health, health behaviours, lifestyles and neighbourhood characteristics. Chapter 8 considered the potential role of health geodemographics in epidemiological studies that traditionally relies on causal models and statistical inference (Figure 36). In this chapter the approach is different and closer to Hand et al.'s definition of data mining;

"The analysis of (often large) observational data sets to find unsuspected relationships and to summarise the data in novel ways that are both understandable and useful to the data owner" (Hand et al., 2001).

What is a good application of health geodemographics? One avenue of research is to design multi-level models, where the effects of residing in a particular neighbourhood type can be estimated relative to other causal factors (Harris et al., 2007). Another approach would be to use geodemographics as a data mining tool following the pragmatic definition of Hand et al (2001). An application is accordingly successful if it is 'understandable and useful to the data owner'.

Healthcare databases, as used in the empirical examples of this thesis, have properties that may make statistical inference difficult - in some cases impossible. Their primary purpose may, e.g. in the case of A\&E attendances or teenager's use of abortion services (see chapter 5 and 6), be administrative or supply side driven. Alternatively the primary purpose may be to create data trails contributing to individual patients' clinical history, e.g. Hospital Episode Statistics (HES).

It may be useful to consider some of the properties where secondary databases stands out from primary research data sets (Anselin et al., 2006; Goodchild, 1986; Hand et al., 2001; Longley et al., 2005; Openshaw, 1991; Winkler, 2006). Primary research data on health typically follow assumptions of linearity, normal distributions, independence, spatial homogeneity, synchrony, 
low-dimensionality, representativeness (no data redundancy) and are collected within controlled, experimental frameworks with predetermined hypotheses of causality. Secondary data sets are in contrast typically;

1) Large and collective;

a. Healthcare databases are centralised and growing; Hospital Episode Statistics is e.g. updated with $12+$ million records each year

b. Data accumulation may lead to data redundancy in both space and time

2) Temporal - Time stamped and frequently updated;

a. Supply side oriented systems are often focussed on transactions and the statistics of service performance. The term data stream may in some cases be more appropriate than the more statically sounding term, data base. Time may also be qualifying in itself, the USATODAY readership classification is e.g. only based on recent visits to their website (section 7.5)

b. Data trails following individual patients as a part of their clinical history, e.g. Hospital Episode Statistics (HES)

3) Spatial - service users and providers are coded with geographical location, e.g. unit postcode. This enables explorative spatial data analysis, spatial data modelling and also contributes to issues of information privacy (chapter 10)

4) High-dimensional - Objects are described by many variables, e.g. the same individual may have many different diagnoses

5) Noisy and asynchronous;

a. Misclassification, data transformation and data transfer errors, mis-alignment errors and partial data loss. Data may be collected, classified and entered by different people in different contexts and for different purposes. Different data sources may subsequently be transformed, reformatted, transferred, merged and mis-aligned or lost (chapter 7). 
b. Indentification of data objects and their deduplication within separate or over several files are additionally complicated by lack of unique and universal identifiers, incomplete data and typographical errors.

c. Asynchronicity; the uncertainty associated with course of events when data is deduplicated and brought together from disparate data sources

6) Non-linear and non-gaussian - Techniques for statistical inference were typically developed for relatively small, sampled data sets before computers became available. Standard statistical tests are as consequence 'always significant' due to sample sizes on the scale of millions rather than tens or hundreds.

Due to the nature of the data bespoke geodemographic systems may offer its owners means of discovering new knowledge about healthcare use in a way that is timely, updateable and crosssectional. The London Hospital Admission Classification (LHAC) could potentially be useful for planning and configuration of hospital services within the region. Planning services according to knowledge of neighbourhoods with rising, complex and intensive healthcare needs could lead to better and more cost-effective healthcare services. NHS are undergoing reforms with new directions for long-term care (Department of Health, 2005b);

1) Locating services closer to home

a. Avoid hospitalisation

b. Improve patients' experience

2) Community care services

a. Community matron to devise individual case management plans

b. Home visits from specialist nurses or health visitors

c. Specialist clinics

3) Primary care services 
a. GPs rewarded for taking on patients with long-term care needs (Quality Outcomes Framework)

Patient with long-term diseases often have intense and complex healthcare needs. Healthcare services are under the new reforms diverted from hospitals to community care services closer to home or indeed at home. Primary Care Trusts now have community matrons that work out case management plans. Mapping these often composite needs will be valuable for the coordination of case management, for locating new community services and for highlighting situations, where patients with mobility problems (e.g. congestive heart failure patients) at present have to travel long distances for routine check-ups.

\subsection{Conclusion}

Bespoke geodemographic classification of large, observational healthcare databases is a promising new technique that can be adapted to a specific healthcare focus. Mapping neighbourhoods with composite, long-term healthcare needs, as demonstrated in this chapter, will be valuable for extending GP services and the planning of new and accessible community services. 


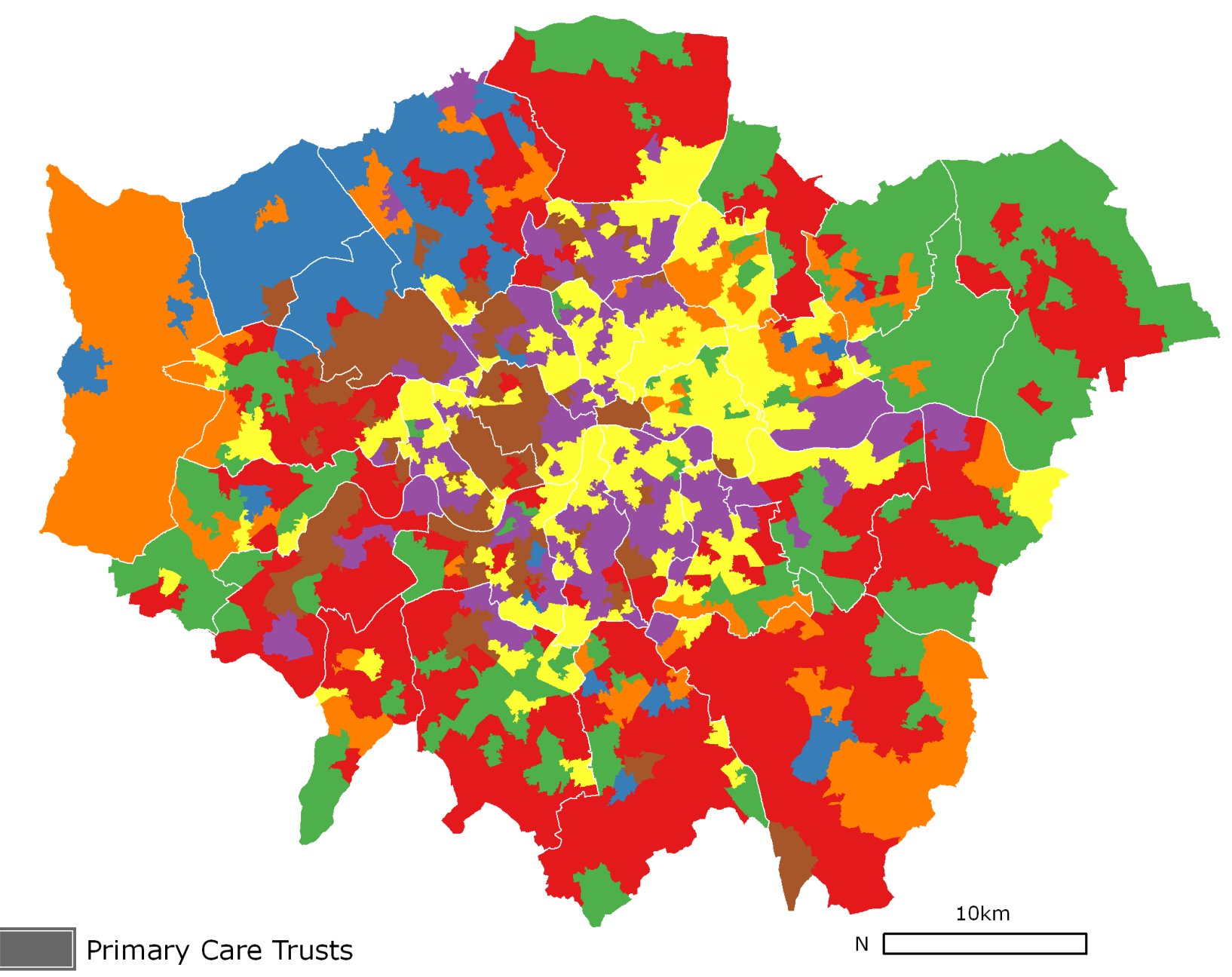

\section{London Hospital Admission Classification}

Typology with above median chronic disease admission rates

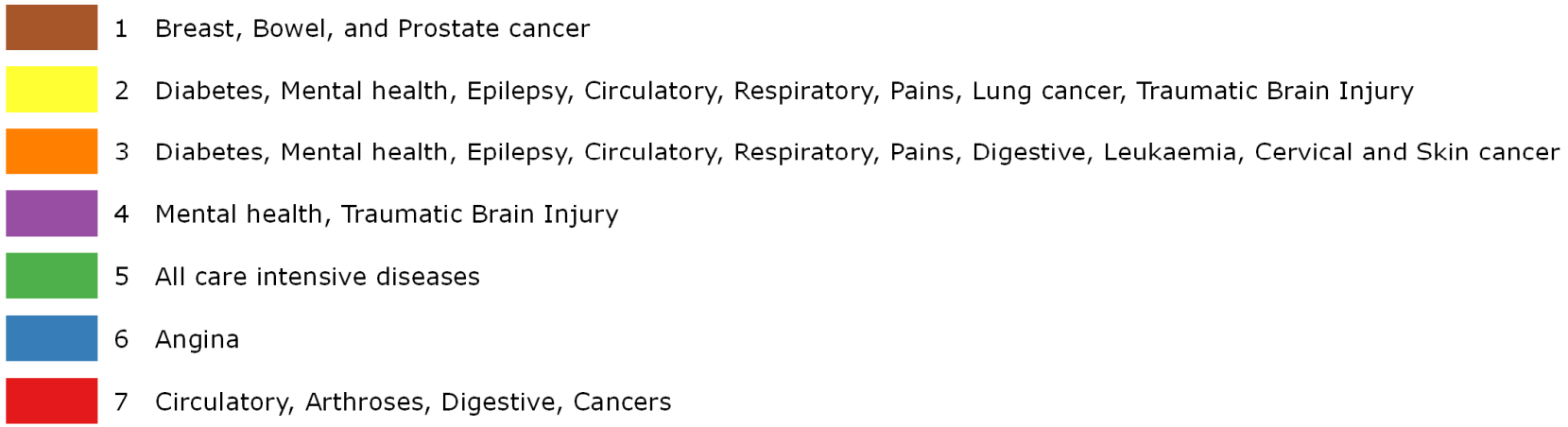

Figure 41 Map of London Hospital Admission Classification 


\section{Chapter 10 Information privacy and secondary data analysis}

Digital representations have come to be the dominant mode of representation. As discussed in chapter 5, digitalisation depends on the formalisation of data and data models (Longley et al., 2005; Schuurman, 2006). The resulting information infrastructures are acknowledged by some as great opportunities for creating functional cities and societies, responding to environmental crises and challenging the social status quo by widening access to public goods (Longley et al., 2005). Others see the rise of information society as centralising, controlling and a way the already advantaged will continue to conspire to further their positions of power and wealth (Goss, 2003; Pickles, 1994). The Internet has become the symbol of the new information infrastructures. Lessig suggests that '[machine] code is law' (Lessig, 1999) and Deleuze asserts that 'physical barriers and constraint within places matter less today than the codes that enable and disable, admit and exclude, accredit and discredit' (Deleuze 1986 in Lyon, 2002).

Information privacy defined as 'the interest an individual has in controlling or influencing the handling of data about themselves' (Clarke, 2006) has to be seen against this backcloth of digitalisation that empowers or exploits. There is in every case good sense in revising communication and information networks critically: what information does organisations and governments hold. What is the justification for collecting this information? Is it secure and what are the risks to privacy?

Lyon explains that 'as the locally-known, embodied person slid from view in the web of social structure, so the importance of credentials, identification and other documentary evidence was amplified' (Lyon, 2002, p. 245). Individuals are categorised and reduced to inert data symbols, a 'digital persona', sufficiently simple to be coded, linked, stored and retrieved (Clarke, 1994). Surveillance thus becomes the means by which governments can assure the now 'estranged' citizenry, tax payers or electorates that public resources are equally or fairly distributed and not 
subject to systematic abuse and free-riding (Boylan, 2004; Nock, 1993). Surveillance in other words preserves trust in a government. It is paradoxically a benign 'Big Brother' that keeps track of people's daily lives and yet enables them to remain private (Lyon, 2002).

\subsection{Healthcare databases and patient consent}

The primary purpose of healthcare databases is to create a data trail, the 'clinical history', of each patient. These primary data are important for future assessments of the patient's condition, prospects and treatment. The collection and storage of the more detailed data are in many places still only stored locally, e.g. in paper form by the patient's family doctor. Information that can be coded, such as diagnoses and prescriptions, are however digitally stored along with patient identifiers, i.e. name, date of birth, organisation code (e.g. NHS number). Data on diseases of public health concern, especially communicative and cancers are stored in central registers with patient consent. All hospital admissions in the UK are likewise stored in a national data warehouse, Hospital Episode Statistics (HES). The analysis of register data can be classified as secondary data analysis: 'uses of confidential patient information ... which [does] not contribute directly to or support the healthcare that a patient receives' (European Union, 2006). There is for different reasons a lot of interest in secondary data analysis because of its potential for evidencebased policy. Compared to other means of gathering information about population health and healthcare use, e.g. surveys, trials, cohorts, etc., secondary data analysis can be cross-sectional, comprehensive, timely and results can be produced at a fraction of the cost of the alternatives.

One of the problems of secondary data analysis is, as mentioned in the introduction, the potential threat to the privacy of individual patients were any data with identifiable information to be released through carelessness or equipment theft and other security breaches. The legislation, e.g. Data Protection Act, notably only protects the data, and not the privacy of the patient directly (Clarke, 2006). The patient is supposed to 'sign away' any doubt and allow their data to be kept 
for research and the 'common good'. Information privacy is a 'hot' topic in the media especially where government sources are involved. Ironically consumers seem surprisingly willing to give up personal and identifiable information to commercial companies - evident from the success of loyalty card schemes. How much is your information privacy reservations worth? A chance to win a holiday or a cardboard token you can exchange against goods in a high street chain store near you (terms and conditions apply)? Perhaps this is due to a difference in what we consider private (medical records) versus what is part of a public persona or lifestyle such as our consumer habits. Privately a patient; publicly a consumer?

The question of patient's express consent ${ }^{11}$ remains salient to the question of information privacy and so do the exemptions that currently enable population wide epidemiological studies; According to the European Standards on Confidentiality and Privacy in Healthcare (2006) it is in effect;

1) "Impracticable to obtain consent for the use of patient information for a secondary use, for example;

a. For a public health study, where the patient information had been obtained some time previously. A possible ground for justifying not obtaining consent would be disproportionate effort; or

b. Obtaining consent for a large sample of patients on whom the information had been obtained many years earlier.

2) Impossible where the confidential information was obtained with consent for a particular secondary use, and the potential for use for another purpose is now being considered. If the data have subsequently been irretrievably decoupled from those who initially gave

\footnotetext{
11 'Express consent' is consent expressed verbally or in writing by a patient in a process where the
} patient has been informed about the nature, purpose and storage of the requested data (European Union, 2006). 
consent, then although they have a moral interest in its further use, gaining their consent to the second use is impossible" (European Union, 2006).

This seems to suggest that 'while we are waiting for new schemes to be fully implemented, we would like research to be continued'. Other crucial safeguards contained in current legislation however are anonymisation, categorisation, aggregation and encryption. For secondary analysis data further have to be anonymised ${ }^{12}$ and access protected by ethical approval procedures in order to ensure that patients' interests are formally represented. It would for instance be unethical if a company would try to obtain hospital records in order to target vulnerable patients with commercial, health-related products.

\subsection{Loss of identifiable data}

Loss of identifiable data from organisations makes sensational newspaper headlines whether it is through stolen or misplaced laptops, CDs lost in the post or the action of 'identity'-stealing hackers (Ohm, 2008). From 2000-2006 a total of 521 incidents were reported by national media in the USA. The number of identifiable records equalled approximately 8 for every American. Slightly more than seven of these were attributable to a single incidence, where a hacker

\footnotetext{
12 ،... it must no longer be possible for anyone to identify the person who is the subject of the data
} directly (that is, from the data itself) or indirectly (that is, from the data itself in conjunction with other data or means that are "reasonably likely to be used", such as an identification number or to one or more factors specific to the subject's physical, physiological, mental, economic, cultural or social identity)' (European Union, 2006). 
'compromised' 1.6 billion records from the marketing services company, Acxiom Corporation ${ }^{13}$ (Erickson \& Howard, 2007). The data consisted of marital status, credit and consumption details for millions of named individuals. Compared to the security concerns within the public sector, this does suggest that another type of data trail, the 'credit history', can be used as a cover for marketing purposes of a rather dubious nature. Whilst the financial sector admittedly has some justification for a primary use of this type of data, it is harder to understand why secondary use by the marketing sector should not be regulated in the same way as for public sources; i.e. following standards for anonymisation, categorisation, aggregation, encryption and data transfer security. Medical records in the same study amounted to about 1 record for every 50 Americans over the period 2000-2006. Most incidents were reported about the commercial sector (36\%), followed by education (32\%), government (21\%), medical (10\%) and the defence sectors $(1 \%)$. The ranking order in which the different sectors appear might be interpreted as inversely correlated with data 'sensitivity' or security efforts; although it may just as well be interpreted as a correlation with the size of the data repositories (not assessed in the paper), their perceived commercial value or 'news' value for the papers reporting it. Erickson and Howard (2007) explain that some but not all US federal states have passed new laws, so that any organisation that experience data breaches now have to inform the individuals whose data have been compromised, i.e. the so-called "Notice of Security Breach". The most important causes reported by Erickson and Howard (2007) were missing or stolen hardware (38\%), the action of hackers $(31 \%)$ and other causes including exposing the data online accounting for the remaining $31 \%$. This suggests that the majority of the security breaches are of an avoidable nature and that it is sometimes quite banal failures that lead to breaches. In the case of the Acxiom Corporation, for instance, the 'hacker' was someone employed by a company that bought online services from Acxiom. He accessed their identifiable records simply by using the same password that had been issued for the limited services that his

${ }^{13}$ Acxiom Corporation supplies customer segmentation and other marketing services discussed in chapter 7. 
company had bought access to. The other major factor, hardware lost or stolen, includes mobile equipment such as laptops. Following incidents in 2007 with lost records from government sources in the UK, e.g. child benefit claimants' financial details, the Information Commissioner's Office (the 'privacy watchdog'), has lately gained new powers to fine organisations that 'deliberately or recklessly commit serious breaches' (Information Commissioner's Office, 2008).

\subsection{A punishing clinical history}

Another issue related to patient privacy can be summed up by Lessig's notion that '[machine] code is law' (1999). Healthcare in the UK is given on the basis of need with a strong emphasis in the profession on the principles of helping (or not harming) patients on contact as discussed in part one under the section headline of principlism (beneficence and non-maleficence). From time to time suggestions of the following nature however surface: to deny e.g. smokers coronary bypass surgery unless they stop smoking (Holland, 2007; Underwood \& Bailey, 1993). Should 'clinical history' be used similarly to another type of data trail, the 'credit history'? If healthcare should be given on the basis of desert and not need; then 'clinical history' contained in primary healthcare data could be used to deny someone treatment - 'code is law'. So far this idea does not seem to be practiced, but it does rightly belong to a debate on privacy that goes beyond designating data that should or should not be shared.

\subsection{Cancer survival}

With the right safeguards in place secondary healthcare data analysis has unique potential to influence health and social policy through timely and low-cost means. This is particularly evident from work with cancer survival within countries and internationally.

The European cancer network has been able to highlight failings in the treatment system of different countries. This type of comparative analysis can hence benefit patients in situations, 
where 'best practice' can be transferred between countries. (Berrino, 2003; Mladovsky et al., 2008).

\subsection{Conclusion}

The storage of secondary data poses particular challenges for patient privacy. The most commonly reported breaches however are avoidable if the current safeguards were enforced. The distinction between primary and secondary data uses here becomes important. For most secondary data purposes relatively simple safeguards, i.e. anonymisation, categorisation, aggregation and encryption would be sufficient to ensure patient confidentiality. The question of patient consent to secondary data analysis is not yet fully implemented; if a high proportion of patients were to opt out, it would have serious consequences for the validity of secondary data research. This emphasises the need to tighten data security and data transfer security for primary purposes as well as enforcing principles of anonymisation for secondary data purposes.

If the current principles of a needs-based healthcare system were to change, clinical history data trails could be used to deny a patient certain treatments, e.g. coronary bypass surgery for smokers. There seems however to be consensus against this at present.

With the right safeguards in place secondary healthcare data analysis has unique potential to influence health and social policy through timely and low-cost means. A prominent example of this is the inequalities exposed in cancer survival across Europe, where patients will benefit, where 'best practice' in effect can be transferred between countries. 


\section{PART THREE: CONCLUSION AND EVALUATION}




\section{Chapter 11 Contribution to social marketing agenda}

If there is one question that repeats itself in each chapter in this thesis, it is the question of balancing individual needs with those of society in the widest sense. Part One explored the fundamental ideas behind public health activity and the ethical and methodological issues that arise when public health campaigns are mounted to target individuals or vulnerable population groups.

The application of social marketing in public health campaigns is based on a premise that there are 'easy-win' endogenous behaviours in groups that given the right, motivational campaigning can influence the target audiences to voluntarily modify or abandon them. Social marketing uses similar terminology to marketing, although there are fundamental differences between the two. The so-called 4Ps of marketing - product, price, place and promotion - can nevertheless still be useful concepts for understanding how a social marketing campaign can be shaped in order to achieve health-promoting behavioural changes. The use of marketing techniques in public health campaigns falls close to manipulation and this potentially flouts ethical principles suggested for public health, i.e. the principle of least restrictive means (principlism, section 1.3). On the other hand social marketing can be justified on the grounds of being counter-manipulation against the over-powering commercial advertising for 'unhealthy' options.

In Chapter 4 three main research questions were identified; a) How are audiences for a given public health objective selected in ways which are scrupulous both ethically and scientifically? b) To which degree does 'selection' also mean 'identification' based on personal information and what are implications to patient privacy? c) To which degree can neighbourhoods, as characterised by geodemographic systems, qualify as target units? 
Chapter 5 discussed why and how campaigns to target individual health behaviours may be justified. It concluded that the majority of population-wide health inequalities, warranting the attention of public health policy, are caused by behavioural, cultural and socio-economic factors. Diseases themselves are however not just social phenomena or purely 'behavioural', but are the outcomes of interactions between both nature and nurture. In this respect neither race nor ethnicity are fully adequate concepts; the former is too crude, the latter is ambiguously defined with regards to any biological effects. Genetic 'identity', as uncovered in new so-called genomewide studies, is not easily categorised, but can be classified using clusters of genetic markers. For a small number of rare diseases there are clear genetic effects, but for most the hereditary component is small or as yet unknown. Traditional race classifications are, in this context, of little practical or scientific importance. It is however still important for public policy to continue recording and examining health inequalities, where these are the product of social processes falling along the lines of coarse-scale ethnic and racial (or 'ethnoracial') groupings. Montoya's self-critical definition of ethnoraces is a useful concept in the monitory of health inequalities as encompassing both behavioural and non-behavioural factors (Montoya, 2007). As a more general point; classification of human difference are used descriptively and attributively in a completely interchangeable fashion in the medical literature (Montoya, 2007). This suggests that wrestling this concept to the ground in order to slap a label on it, may be a wholly futile project, because nature and nurture rarely can be dissociated in health studies. Ethnoraces would therefore be a more honest reflectance of this inherent ambiguity.

The self-assigned ethnicity categories used in the Census and by NHS are - when not meticulously collected - close to the race concept of distinguishing individuals mainly by skin colour and do not as such convey much sense of any social or cultural context for local health inequality studies. Names based classification of ethnicity is therefore promising for database studies of routinely collected data. Names-based ethnicity only represents one of many dimensions in the construction of identity and ethnicity. Cross-tabulating birthplace and names- 
based ethnicity, for instance, gave greater insight into the determining factors in low breast screening uptake. Names-based classification of ethnicity can nevertheless give a rapid assessment of inequalities among healthcare users as demonstrated empirically in the example of GP registration or equalities in the frequent use of $A \& E$ for minor incidents.

Health and healthcare utilisation varies across ethnic groups and local healthcare authorities have an obligation to mitigate health inequalities, improve local environments and empower individuals to choose healthier options. Ethnicity is however a very sensitive topic in public health decision-making that seems most justified when the target population stand to benefit from the campaign aims. One example is inequalities in breast screening uptake; if a health authority choose not to act, the consequence of this inaction would be that women from ethnic minorities would have a higher mortality from a progressive disease such as breast cancer. One the other hand, if a health authority targets an ethnic minority in order to reduce demand on A\&E, because this target 'fits' popular perception or prejudice, it is inevitably a more dubious act. There are clearly immense challenges in developing purposeful 'tools' for evidence-based public health. The example 'tools' presented here are a part of this development, although the ethical dilemmas they unearth will continue to require careful and balanced decisions - they must in other words be 'tools and not rules' (Rawlins, 2008) ${ }^{14}$.

The potential role of public and private organisations in fostering behavioural changes was discussed in Chapter 6, which concerned efforts to reduce teenage pregnancy in Southwark PCT. Interventions to reduce unwanted teenage pregnancy have been met with social criticism which can be summarised as state interference that lacks respect for the autonomy of the individual. Some also hold that teenage motherhood should be approached with a 'normality by locality'

\footnotetext{
${ }^{14}$ In reference to the use of QALYs in decisions over NHS's funding of new medicines
} 
view. Conversely, interventions may be seen as a way of creating greater autonomy or positive freedom for the same individuals. In a liberal and pluralistic society citizens must be 'free to make choices' about how they would like to lead their lives. Those undergoing pregnancy in their teens are nevertheless more likely to drop out of school and experience diminished life chances as a consequence. Surveillance of sexual health is charged with privacy issues and evidence-based interventions must accordingly be designed with great sensitivity. The unusually high and persistent teenage pregnancy rates in some parts of Southwark PCT suggests that new campaigns should be targeted not only at areas, for which data are currently available (Census wards), but also consider the specific settings, such as secondary schools and GP practices, that are most likely to be in contact with teenagers at the greatest risk. The traditional view of service-user geography is that "Services [are] described in terms of fixed locations often providing for territorial jurisdictions, [where] distance decay models describe varying utility in space". A relational view would instead recognise that services are "layered in their availability to populations via varying paths in time and space. Euclidian distance may not be relevant to utility" (Cummins et al., 2007). Both secondary schools and GP practices are to a degree chosen by users and the Census areas, for which evidence currently exists, are for that reason rarely coterminous with the principal catchment areas of those services (Gibin et al., 2007; Glennerster, 1991; Harris \& Johnston, 2008). Hotspot maps based on density surfaces can be valuable for the location of many of the essential sexual health services requested by teenage users in surveys. Density surfaces and scan statistics (e.g. Openshaw's (1987) Geographical Analysis Machine) have the advantage of highlighting areas of greater risk and at the same time protect privacy by obscuring the exact location of individual users. Postcodes or other fine-scale geographical units can serve as a nexus for anonymous coupling of health and education data to estimate conception rates for local schools or GP surgeries. Estimated teenage conception rates for local secondary schools proved to be effective in engaging target school managements in new sexual health campaigns. 
The campaign, in other words, had to recognise the role of facilitators in social marketing similarly to Khan's accessibility model for healthcare utilisation (Kahn, 1992).

Chapter 7 discussed the targeting of public health campaigns based on areal aggregations, i.e. geographical areas or neighbourhood type aggregations. Geodemographics is primarily a data mining tool for the discovery of the complex contexts in which many health outcomes unfold within a society in 'real-time'. Compared to the much used uni-directional deprivation scores in health inequality studies, geodemographics attempts to model the multi-dimensional nature of neighbourhood differences with indicators ranging from tenure, age structure and ethnic composition to income scale and consumption patterns.

Geodemographic targeting of public health campaigns may, however, not necessarily always be the most efficient compared to alternative means of geographical targeting (Chapter 8). Even if two analysts chose the same indicators and clustered the neighbourhoods using the same algorithm they would still likely derive two different geodemographic systems because of the difficulties in standardising the rules for choosing the optimal number of clusters at different hierarchical levels. Citizens may as a consequence be selected and deselected for interventions because they live in the 'wrong postcode' or the wrong 'neighbourhood type'. Two different criteria for validation of geodemographic systems are proposed; a) sensitivity of geodemographic targeting relative to geographic targeting; b) number-to-target as the included base population divided by the number of correctly targeted cases in the target area. Following these two criteria, data for hospital admissions for common long-term diseases in Greater London showed the sensitivity of geodemographic systems to be consistently low and below $50 \%$. Considering the cost-increases from including non-target base populations in the target area, geodemographic systems would again be more costly to deploy than geographic targeting schemes. Postcode geodemographic systems had the lowest sensitivity, but would be less costly to deploy than 
geodemographic systems at higher levels of aggregation, due to the lower number-to-target figures.

The regional geodemographic system seems a promising addition to the geodemographic 'family' of planning and social research tools (Chapter 8). The prime advantage of geodemographic systems is that the segments as representations are relatively homogenous in socio-economic terms and thus becomes the mirror distortion of different lifestyles (Webber \& Longley, 2003). A regional classification will in this sense always be more relevant to a local context. With regards to the health domain many surveys are nonetheless based on a national sampling frame and hence will continue to need national classifications for their interpretation. In time decision makers may come to appreciate the value of different geodemographic classifications for different purposes; some national and some regional or local.

The value of age-standardisation was evident especially from geodemographic profiles of long-term diseases associated with elderly patients (chapter 8). Elderly with poorer health would for instance be 'hidden' if residing in areas with a younger-than-average age structure. Suburban neighbourhoods would in the same example 'qualify' for targeting, although the implied association with 'upstream' policy variables would be confounded by differences in the base population age structure alone.

The analyses of routinely collected data of health outcomes and health behaviours have to acknowledge and address a number of potential biases. Different population groups noticeably leaves fewer traces than others in the digitalised society. Chapter 7 explored some of these in the context of bias in systematic inference (section 7.3). In addition to widely known biases Chapter 7 also pointed to biases related to population dynamics that lifecourse cohort studies classically attempt to circumvent by following individuals through a matrix of varying exposures in time and 
space. Biases reflecting these dynamics are e.g. settler-wanderer bias, which asserts that individuals that reside for longer in an area are more likely to be registered at all or multiply and so information and inferences made from secondary data sources are more likely to be based on the 'settler' proportion of the population although they may not be representative for the de facto population in a region at a given time. Another bias relates to the fact that the private healthcare market has grown to represent an estimated third of the healthcare expenditure nationally (Clarke et al., 2008; Department of Health, 2005a). Apart from birth and death the data from patients seeking treatment privately are not recorded centrally and this may lead to a bias in the interpretation of secondary health data; a cash and care bias. Bias from incomplete and differential data recording was furthermore evident from the breast screening study (section 5.3), e.g. the varying propensity for patients to give birthplace information by age, time and ethnicity.

Bespoke geodemographic classification of large, observational healthcare databases is a promising new technique that can be adapted to a specific healthcare focus (Chapter 9). The use of geodemographics to understand causality in an epidemiological paradigm is burdened with many assumptions. Data mining of healthcare service demands make fewer assumptions and can be an interesting way to deal with large and complex data sets. Mapping neighbourhoods of composite, long-term healthcare needs, for example, could be valuable for extending GP services and the planning of new and accessible community services.

Ultimately, geodemographic systems may be useful for describing (or profiling) current regional or national trends in health inequalities. Their use for mapping of local healthcare needs or use as evidence of causality is however limited by their ecological design. The national healthcare service (NHS) is, compared to most of the marketing industry, in the superior position of having the organisation and means to maintain detailed records of demand. The use of geodemographics would in the context of evidence-based public health offer inferior solutions to 
what could be achieved from exploring the vast and accumulating data repositories already available to planners within the NHS.

Secondary data analysis has unique potentials in identifying important health inequalities, health behaviours and may further improve patients' treatment, e.g. through evaluation of cancer survival internationally (Chapter 10). Many health outcomes have complex epidemiologies that require identifiable, individually linked and chronologically enforced data. In order to a minimise concomitant threat to individual privacy, protocols for data anonymisation, categorisation, aggregation, encryption and data transfer security are routinely implemented prior to secondary data use. Reported breaches of data security suggest that these at present mainly pertain to human error and could thus be reduced significantly if existing security measures were enforced. If a high proportion of patients were to opt out of secondary data schemes it would have serious consequences for the validity of secondary data research. This emphasises the need to tighten data and data transfer security for primary purposes as well as enforcing protocols for anonymisation of secondary data.

The aim of this thesis has been to explore and ultimately answer questions related to the selection and targeting of audiences in public health campaigns. As an industry-based or applied research project it would have been satisfying to deliver all answers 'on the back of a postcard'. Many of the proposed tools are reassuringly simple. The use of scientific tools does however not remove the fact that health and public health ultimately are value-laden concepts and that public health decision-makers are faced with challenging ethical dilemmas that require sophisticated and balanced decisions. The thesis may on this note conclude with one of the pioneers of modern public health, Charles Winslow, that public health is invariably 'a science and an art' (Winslow, 1920). 


\section{References}

Aday, L.A., \& Andersen, R. (1974). A framework for the study of access to medical care. Health Services Research, 9(3), 208-220.

Agran, P.F., Anderson, C., Winn, D., Trent, R., Walton-Haynes, L., \& Thayer, S. (2003). Rates of Pediatric Injuries by 3-Month Intervals for Children 0 to 3 Years of Age. Pediatrics, 111(6), e683-692.

Anselin, L., Syabri, I., \& Kho, Y. (2006). GeoDa: an introduction to spatial data analysis. Geographical Analysis, 38(1), 5-22.

Arai, L. (2007). Peer and neighbourhood influences on teenage pregnancy and fertility: qualitative findings from research in English communities. Health and Place, 13(1), 87-98.

Atkinson, P., \& Unwin, D.J. (2002). Density and local attribute estimation of an infectious disease using MapInfo. Computers and Geosciences, 28(11), 1095 1105.

Barnet, B., Arroyo, C., Devoe, M., \& Duggan, A.K. (2004). Reduced School Dropout Rates Among Adolescent Mothers Receiving School-Based Prenatal Care. Archives of Pediatric and Adolescent Medicine, 158(3), 262-268.

Barreiro, L.B., Laval, G., Quach, H., Patin, E., \& Quintana-Murcia, L. (2008). Natural selection has driven population differentiation in modern humans. Nature Genetics, 40(3), 340-345.

Barrett, G., Cassell, J.A., Peacock, J.L., \& Coleman, M.P. (2006). National survey of British public's views on use of identifiable medical data by the National Cancer Registry. British Medical Journal, 332(7549), 1068-1072.

Batty, M. (2006). Rank clocks. Nature, 444(7119), 592-596.

Beauchamp, D.E., \& Childress, J.F. (2001). Principles of Biomedical Ethics Oxford: Oxford University Press

Bennett, S.E., \& Assefi, N.P. (2005). School-based teenage pregnancy prevention programs: a systematic review of randomized controlled trials. Journal of Adolescent Health, 36, 72-81.

Berners-Lee, T., Hendler, J., \& Lassila, O. (2001). The semantic web - the new for of web content that is meaningful to computers will unleash a revolution of new possibilities. Scientific American, May 2001.

Berrino, F. (2003). The EUROCARE Study: strengths, limitations and perspectives of population-based, comparative survival studies. Annals of Oncology, 14(5), v913.

Besley, T., \& Kanbur, R. (1990). The principles of targeting. Policy Research Working Paper Series, 385.

Beyer, E.L. (2004). Hawth's analysis tools for ArcGIS

Bhopal, R. (1997). Is research into ethnicity and health racist, unsound, or important science? British Medical Journal, 314(7096), 1751-. 
Blaxter, M. (2003). Biology, social class and inequalities in health - their synthesis in 'health capital'. In S.J. Williams,L. Birke, \& G.A. Bendelow (Eds.), Debating Biology: Sociological Reflections on Health, Medicine, and Society. London: Routledge.

Bleich, S., Cutler, D., Murray, C., \& Adams, A. (2008). Why Is the Developed World Obese? Annual Review of Public Health, 29, 273-295.

Bodenheimer, T., Wagner, H., \& Grumbach, K. (2002). Improving primary care for patients with chronic illness: the chronic care model, part 2. JAMA, 288, 19091914.

Bonell, C., Fletcher, A., \& McCambridge, J. (2007). Improving school ethos may reduce substance misuse and teenage pregnancy. British Medical Journal, 334, 614-616.

Boorse, C. (1975). On the distinction between disease and illness. Philosophy and Public Affairs, 5(1), 49-68.

Boylan, M. (2004). Public health policy and ethics Dordrecht: Kluwer Academic Publishers

Brainard, J., Jones, A., Bateman, I.J., \& Lovett, A.A. (2006). Equal access for all? Ethnicity and public park availability in Birmingham (UK). CSERGE Working Paper, 06-05.

Braun, L., Fausto-Sterling, A., Fullwiley, D., Hammonds, E.M., Nelson, A., Quivers, W., Reverby, S.M., \& Shields, A.E. (2007). Racial categories in medical practice: how useful are they? PLoS Medicine, 4(9), 1423-1428.

Brodeur, J., Bedard, Y., Edwards, G., \& Moulin, B. (2003). Revisiting the concept of geospatial interoperability within the scope of human communication processes. Transactions in GIS, 7(2), 243-265.

Bulmer, M. (1996). The ethnic group question in the 1991 Census of Population. In D. Coleman, \& J. Salt (Eds.), Ethnicity in the 1991 Census. Volume 1. Demographic characterisitics of the ethnic minority populations (pp. xi-xxix). London: Office for National Statistics, HMSO.

Burrows, R., \& Gane, N. (2006). Geodemographics, Software and Class. Sociology, 40(5), 793-812.

Butt, D., Jani, N., \& Petrie, S. (2006). Mapping of hotspots: An analysis of teenage conceptions in Southwark 2002-04: Public Health, Southwark PCT.

Callingham, M. (2006). Exploring the Use and Value of the ONS output area classification. Royal Society of Statistics: Office for National Statistics.

Carpenter, J.R., Kenward, M.G., \& White, I.R. (2007). Sensitivity analysis after multiple imputation under missing at random: a weighting approach. Statistical Methods in Medical Research, 16(3), 259-275.

Chandola, T., Bartley, M., Sacker, A., Jenkinson, C., \& Marmot, M. (2003). Health selection in the Whitehall II study, UK. Social Science and Medicine, 56(10), 2059-2072.

Chaney, D. (1996). Lifestyles London: Routledge

Childress, J.F., Faden, R.R., \& Gaare, R.D. (2002). Public health: mapping the terrain. Journal of Law, Medicine and Ethics, 30, 170-178.

Cho, W.K.T., \& Manski, C.F. (2008). Cross-level/Ecological Inference. In J. BoxSteffensmeier,H. Brady, \& D. Collier (Eds.), Oxford Handbook of Political Methodology (pp. 547-569). Oxford: Oxford University Press. 
Choi, B.C.K. (2000). Bias overview. In M.H. Gail, \& J. Benichou (Eds.), Encyclopedia of epidemiological methods (pp. 74-82). Chichester: Wiley.

Clapham, D. (2006). Identity and Lifestyle in Housing Research, ENHR. Ljubljana: Urban Planning Institute of the Republic of Slovenia.

Clarke, C., Stone, T., \& Downey, A. (2008). Achieving the potential - how best to pay for ever higher expectations of our public services. London: Klynveld, Peat, Marwick and Goerdeler Limited Liability Partnership.

Clarke, R. (1994). The Digital Persona and its Application to Data Surveillance. The Information Society, 10(2).

Clarke, R. (2006). Introduction to Dataveillance and Information Privacy, and Definitions of Terms: Department of Computer Science, Australian National University.

Cochrane, A. (1972). Effectiveness and efficiency: Random reflections on health services London: Nuffield Provincial Hospitals Trust

Cowan, J. (2006). Supporting Human Rights Unconditionally is Misguided Moralism. Anthropology News, 47(7).

Cressie, N. (1991). Statistics for spatial data New York: J. Wiley \& Sons, Inc.

Crosnoe, R., \& McNeely, C. (2008). Peer relations, adolescent behavior, and Public Health research and practice. Family and Community Health, 31(18), S71-S80.

Cummins, S. (2007). Commentary: Investigating neighbourhood effects on health-avoiding the 'Local Trap'. International Journal of Epidemiology, 36(2), 355-357.

Cummins, S., Curtis, S., Diez-Roux, A.V., \& MacIntyre, S. (2007). Understanding and representing 'place' in health research: a relational approach. Social Science and Medicine, 65, 1825-1838.

Davey Smith, G. (2003). Health Inequalities - lifecourse approaches Bristol: The Policy Press

Dawson, A., \& Verweij, M. (2007). Ethics, prevention and public health Oxford: Clarendon Press

de Smith, M.J., Goodchild, M.F., \& Longley, P.A. (2008). Geospatial Analysis Leicester: Matador

Deer, B. (2006). How a spurious health scare brought an old killer back, The Sunday Times. London.

Delgado-Rodriguez, M., \& Llorca, J. (2004). Bias. Journal of Epidemiology and Community Health, 58(8), 635-641.

Department of Children Schools and Families (2007). The Children's Plan - building brighter futures. London.

Department of health (1999). Saving lives: our healthier nation. London: Department of Health.

Department of Health (2000). The NHS Plan: a plan for investment, a plan for reform. London.

Department of Health (2003). NHS Confidentiality Code of Practice, Best Practice Guidance. London.

Department of Health (2004a). Practice Based Commissioning; Promoting Clinical Engagement.

Department of Health (2004b). Improving chronic disease management. London: Department of Health. 
Department of Health (2004c). Choosing health: making healthier choices easier. London.

Department of Health (2005a). Primary Care Trust Recurrent Revenue Allocations 2006$07 \&$ 2007-08: Department of Health.

Department of Health (2005b). National Service Framework for Long-term Conditions. London: Department of Health.

Department of Health (2006). Our Health, Our Care, Our Say: A New Direction for Community Services. London: Department of Health.

Department of Health and Social Security (1980). Inequalities in health (The Black Report). London.

DiCenso, A., Borthwick, V.W., Busca, C.A., Creatura, C., Holmes, J.A., \& Kalagian, W.F. (2001). Completing the picture: adolescents talk about what's missing in sexual health services. Canadian Journal of Public Health, 92, 35-38.

DiCenso, A., Guyatt, G., Willan, A., \& Griffith, L. (2002). Interventions to reduce unintended pregnancies among adolescents: systematic review of randomised controlled trials. British Medical Journal, 324, 1426-1430.

Dictionary.com (2008). The American Heritage Dictionary of the English Language. Boston: Houghton Miffin Co.

Dobrow, M.J., Goel, V., \& Upshur, R.E.G. (2004). Evidence-based health policy: context and utilisation. Social Science \& Medicine, 58, 207-217.

Drezner, D.W. (2007). All politics is global Princeton: Princeton University Press

Entwistle, B. (2007). Putting people into place. Demography, 44(4), 687-703.

Entwistle, B., Faust, R.R., \& Kaneda, T. (2007). Networks and Contexts: Variation in the Structure of Social Ties. American Journal of Sociology, 112(5), 1495-1533.

Erens, B., Primatesta, P., \& Prior, G. (1999). Health survey for England: the health of ethnic groups London: The stationary office

Erickson, K., \& Howard, P. (2007). A case of mistaken identity? News accounts of hacker, consumer, and organizational responsibility for compromised digital records. Journal of Computer-Mediated Communication, 12(4).

European Union (2006). European Standards on Confidentiality and Privacy in Healthcare. Bruxelles.

Flowerdew, R., \& Leventhal, B. (1998). Under the microscope. New Perspecitives, 18, 36-38.

Fotheringham, A.S. (2000). A bluffer's guide to A Solution to the Ecological Inference Problem. Association of American Geographers.

Free, C., White, P., Shipman, C., \& Dale, J. (1999). Access to and use of out-of-hours services by members of Vietnamese community groups in South London: a focus group study. Family Practice, 16(4), 369-374.

Friendly, M. (2001). A brief history of the Mosaic display Toronto: Department of Psychology, York University

Fullwiley, D. (2007). The Molecularization of Race: Institutionalizing Human Difference in Pharmacogenetics Practice. Science as Culture, 16(1), 1 - 30.

Gardner, M.N., \& Brandt, A.M. (2006). "The Doctors' Choice Is America's Choice": The Physician in US Cigarette Advertisements, 1930-1953. American Journal of

Public Health, 96(2), 222-232. 
Gavrilov, L., \& Gavrilova, N. (2001). The Reliability Theory of Aging and Longevity. Journal of Theoretical Biology, 213, 527-545.

Gessen, M. (2007). Blood matters - a journey along the genetic frontier London: Granta Publications

Gibin, M., Longley, P., \& Atkinson, P. (2007). Kernel density estimation and percent voulme contours in General Practice catchment area analysis in urban areas. In

A.C. Winstanley (Ed.), Geographical Information Science Research UK Conference pp. 270-276). Maynooth, Ireland.

Giesen, P., Franssen, E., Mokkink, H., \& Bosch, W. (2006). Patients either contacting a general practice cooperative or accident and emergency department out of hours: a comparison. Emergency Medicine Journal, 23, 731-734.

Gigerenzer, G. (2002). Reckoning with risk - learning to live with uncertainty London: Allen Lane The Penguin Press

Gill, L. (1999). OX-LINK: The Oxford Medical Record Linkage System, Record Linkage Techniques 1997 pp. 15-33). Washington DC: National Academy Press.

Gill, P.S., Bhopal, R., Wild, S., \& Kai, J. (2005). Limitations and potential of country of birth as proxy for ethnic group. British Medical Journal, 330(7484), 196-a-.

Glennerster, H. (1991). Quasi-markets for education? Economic Journal, 101, 12681276.

Gomez-Rubio, V., Ferrandiz-Ferragud, J., \& Lopez-Quilez, A. (2005). Detecting clusters of disease with R. Journal of Geographical Systems, 7, 189-206.

Goodchild, M.F. (1986). Spatial autocorrelation Norwich: GeoBooks

Gordis, L. (2004). Epidemiology Philadelphia: W.B. Saunders

Goss, J. (1995). Marketing the new marketing: the strategic discourses of GIS. In J. Pickles (Ed.), Ground truth: The social implications of GIS (pp. 130-170). New York: Guilford Press.

Goss, J. (2003). We know where you are and we know where you live. In D.B.

Clarke,M.A. Doel, \& K.M.L. Housiaux (Eds.), The consumption reader (pp. 211216). London: Routledge.

Government Gazette (1950). Population Registration Act 30, Republic of South Africa.

Graham, S.D.N. (2005). Software-sorted geographies. Progress in Human Geography, 29(5), 562-580.

Gray, A. (2005). Population ageing and health care expenditure. Ageing Horizons, 2, $15-$ 20.

Grossman, M. (1972). The Demand for Health: A Theoretical and Empirical Investigation New York: Columbia University Press

Haines, M.R. (1999). The great modern mortality transition, Social Science History Association. Fort Worth, Texas.

Haining, R. (2003). Spatial Data Analysis Cambridge: Cambridge University Press

Hand, D., Mannila, H., \& Smyth, P. (2001). Principles of Data Mining Cambridge MA: MIT Press

Harris, J. (2005). It's not NICE to discriminate. Journal of Medical Ethics, 31(7), 373375.

Harris, R., Sleight, P., \& Webber, R. (2005). Geodemographics, GIS and neighbourhood targeting Chichester: John Wiley \& Sons Ltd. 
Harris, R., Johnston, R., \& Burgess, S. (2007). Neighbourhoods, ethnicity and school choice: develping a statistical framework for geodemographic analysis. Population Research and Policy Review, 26, 553-579.

Harris, R., \& Johnston, R. (2008). Primary Schools, Markets and Choice: Studying Polarization and the Core Catchment Areas of Schools Applied Spatial Analysis and Policy, 1, 59-84.

Hart, J.T. (1971). The inverse care law. Lancet, 1(7696), 405-412.

Harvey, D. (2005). A brief history of neoliberalism Oxford: Oxford University Press

Haynes, R.M., Lovett, A.A., Bentham, G., Brainard, J.S., \& Gale, S.H. (1995). Comparison of ward population estimates from FHSA patient registers with the 1991 Census. Environment and Planning A, 27(11), 1849-1858.

Higgs, G., \& Gould, M. (2001). 'Is there a role for GIS in the 'new NHS'? Health and Place, 7, 247-259.

Holcombe, R.G. (1997). A theory of the theory of public goods. Review of Austrian Economics, 10(1), 1-22.

Holland, S. (2007). Public Health Ethics Cambridge: Polity Press

Hooker, B. (2008). Rule consequentialism. In N. Zalta (Ed.), The Stanford Encyclopedia of Philiosophy.

Hsiao, W.C., \& Heller, P.S. (2007). What macroeconomists should know about health care policy Washington DC: International Monetary Fund

Hursthouse, R. (2008). Virtue ethics. In N. Zalta (Ed.), The Stanford Encyclopedia of Philiosophy.

Information Commissioner's Office (2008). ICO welcomes new powers to fine organisations for data breaches. London.

Jennings, B. (2007). Public health and civic republicanism: toward an alternative framework for public health. In A. Dawson, \& M. Verweij (Eds.), Ethics, Prevention, and Public Health. Oxford: Clarendon Press.

Johansen, A., Rasmussen, S., \& Madsen, M. (2006). Health behaviour among adolescents in Denmark: Influence of school class and individual risk factors. Scandinavian Journal of Public Health, 34(1), 32-40.

Jones, S. (1996). Don't blame the genes, Guardian. London.

Joseph, A., \& Phillips, D.R. (1984). Accessibility and utilization: geographical perspectives on health care delivery London: Harper \& Row

Jung, C.G. (1928). The Relations between the Ego and the Unconscious. In H. Read,M. Fordham, \& G. Adler (Eds.), The collected works of C. G. Jung. Princeton: Princeton University Press 1983.

Kahn, A.A. (1992). An integrated approach to measuring potential spatial access to health care services. Socio-eonomic Planning Sciences, 26(4), 275-287.

Kahn, J.D. (2007). Race-ing Patents/Patenting Race: An Emerging Political Geography of Intellectual Property in Biotechnology. Iowa Law Review, 92(2), 353.

Katsanis, S.H., Javitt, G., \& Hudson, K. (2008). A Case Study of Personalized Medicine. Science, 320(5872), 53-54.

Kaufman, L., \& Rousseeuw, P.J. (2005). Finding groups in data - an introduction to cluster analysis New Jersey: John Wiley \& Sons, Inc.

King, G., Rosen, O., \& Tanner, M.A. (2004). Information in Ecological Inference: an introduction. In G. King,O. Rosen, \& M.A. Tanner (Eds.), Ecological Inference - 
New Methodological Strategies (pp. 1-12). Cambridge: Cambridge University Press.

Kirkwood, B.R., \& Sterne, J.A.C. (2003). Essential Medical Statistics Malden: Blackwell Science

Kotler, P., \& Zaltman, G. (1971). Social Marketing: An approach to planned social change. Journal of Marketing, 35, 3-12.

Kotler, P., Roberto, N., \& Lee, N. (2002). Social marketing: Improving the quality of life London: SAGE

Lalonde, M. (1974). A new perspective on the health of Canadians. Ottawa: National Ministry of Health and Welfare.

Law, I., \& Widdows, H. (forthcoming). Conceptualising health: insights from the capability approach. Health Care Analysis.

Leichter, H.M. (1991). Free to be foolish. Politics and health promotion in the USA and Great Britain Princeton: Princeton University Press

Lessig, L. (1999). Code and the other laws of cyberspace New York: Basic Books

Lewis, R., \& Dixon, J. (2004). Rethinking management of chronic diseases. BMJ, 328, 220-222.

Lipsett, A. (2008). 'They are the indicators that schools can't be responsible for' (30 April 2008), Guardian. London.

Long, J.S., \& Freese, J. (2006). Regression models for categorical dependent variables using Stata College Station: Stata Press

Longley, P.A. (2005). A renaissance of geodemographics for public service delivery. Progress in Human Geography, 29, 57-63.

Longley, P.A., Goodchild, M.F., Maguire, D.J., \& Rhind, D.W. (2005). Geographic information systems and science Chichester: John Wiley \& sons, ltd.

Lovett, A.A., Haynes, R., Sunnenberg, G., \& Gale, S. (2002). Car travel time and accessibility by bus to general practioner services: a study using patient registers and GIS. Social Science \& Medicine, 55, 97-111.

Lyon, D. (2002). Everyday surveillance - personal data and social classifications. Information, Communication and Society, 5(2), 242-257.

Lyon, D. (2006). Surveillance, Power and Everyday Life, Oxford Handbook of Information and Communication Technologies (pp. 1-37). Oxford: Oxford University Press.

Macintyre, S., \& Ellaway, A. (2003). Neighbourhoods and Health: Overview, Neighbourhoods and Health (pp. 20 - 42).

Macintyre, S. (2007). Deprivation amplification revisited; or, is it always true that poorer places have poorer access to resources for healthy diets and physical activity? International Journal of Behavioral Nutrition and Physical Activity, 4(1), 32.

MacLean, S. (2007). Health in development. In A. McGrew, \& N.K. Poku (Eds.), Globalization, development and human security (pp. 132-151). Cambridge: Polity Press.

Manski, C.F. (1995). Identification problems in the social sciences Cambridge MA: Harvard University Press

Manski, C.F. (2003). Partial identification of probability distributions New York: Springer 
Mateos, P., Jones, C.E., Longley, P., \& Webber, R. (2005). Data Mining of ethnic information in patient records through birthplace \& name analysis. An example in Camden PCT, Royal Geographic Society with Institute of British Geographers (RGS-IBG) annual conference. London.

Mateos, P. (2007a). An ontology of ethnicity based upon personal names ( $\mathrm{PhD}$ thesis): Department of Geography, University College London.

Mateos, P. (2007b). A review of name-based ethnicity classification methods and their potential in population studies. Population, Space and Place, 13(4), 243-263.

Mateos, P., Webber, R., \& Longley, P. (2007). The cultural, ethnic and linguistic classification of populations and neighbourhoods using personal names. CASA Working Paper, 116, 1-75.

McAuley, J., De Souza, L., Sharma, V., Robinson, I., Main, C.J., \& Frank, A.O. (1996). Self defined ethnicity is unhelpful. British Medical Journal, 313(7054), 425b-426.

McCamley-Finney, A., \& McFadden, M. (1999). Qualitative studies in health promotion. In E.R. Perkins,I. Simnett, \& L. Wright (Eds.), Evidence-based Health Promotion (pp. 353-373). Chichester: John Wiley \& Sons.

McCarthy, M. (2002). A brief history of the World Health Organization. Lancet, 360, 1111-1112.

McCarthy, M.I., Abecasis, G.R., Cardon, L.R., Goldstein, D.B., Little, J., Ioannidis, J.P.A., \& Hirschhorn, J.N. (2008). Genome-wide association studies for complex traits: consensus, uncertainty and challenges. Nature Review Genetics, 9(5), 356369.

McGilchrist, M., Sullivan, F., \& Kalra, D. (2007). Assuring the confidentiality of shared electronic health records. British Medical Journal, 335(7632), 1223-1224.

Michael, J. (2008). Race. In N. Zalta (Ed.), The Stanford Encyclopedia of Philosophy Palo Alto: Stanford University.

Mill, J.S. (1975). Three essays: on Liberty, Representative Government, The Subjection of Women Oxford: Oxford University Press

Mladovsky, P., Mossialos, E., \& McKee, M. (2008). Improving access to research data in Europe. British Medical Journal, 336(7639), 287-288.

Montoya, M. (2007). Bioethnic conscription: Genes, Race, and Mexicana/o Ethnicity in Diabetes Research. Cultural Anthropology, 22(1), 94-128.

Morgenstern, H., \& Thomas, D. (1993). Principles of study design in environmental epidemiology. Environmental Health Perspectives, 101(S4), 23-38.

Moss, S., \& Blanks, R. (1998). Calculating appropriate target cancer detection rates and expected interval cancer rates for the UK NHS Breast Screening Programme. Interval Cancer Working Group. Journal of Epidemiology and Community Health, 52(2), 111-115.

Muir Gray, J.A. (1996). Evidence-based healthcare Kidlington: Churchill Livingstone

Murphy, A.W. (1998a). 'Inappropriate' attenders at accident and emergency departments I: definition, incidence and reasons for attendance. Family Practice, 15(1), 23-32.

Murphy, A.W. (1998b). 'Inappropriate' attenders at accident and emergency departments II: health service responses. Family Practice, 15(1), 33-37.

Musgrove, P. (2003). Health economics in development Washington DC: World Bank

Nader, L. (2006). Human rights and moral imperialism - a double-edged story. Antropology News, 47(9), 6. 
Naish, J. (1994). Intercultural consultations: investigating factors that deter non English speaking women from attending their general practioners for cervical screening. British Medical Journal, 309, 1126-1129.

Nanchahal, K., Mangatani, P., Alston, M., \& Dos Santos Silva, I. (2001). Development and validation of a computerized South Asian names and group recognition algorithm (SANGRA) for use in British health-related studies. Journal of Public Health Medicine, 23(4), 278-285.

Nebert, D.W., Zhang, G., \& Vesell, E.S. (2008). From human genetics and genomics to pharmacogenetics and pharmacogenomics: past lessons, future directions. Drug Metabolism Reviews, 40(2).

NICE (2008). National Institute for Clinical Excellence (accessed 9 November 2008). London.

Noble, M., Wright, G., Dibben, C., Smith, G., McLennan, D., Anttila, C., Barnes, H., Mokhtar, C., Noble S, Avenell, D., Gardner, J., Covizzi, I., \& Lloyd, M. (2004). Indices of Deprivation 2004. London: Office of the Deputy Prime Minister.

Nock, S.L. (1993). The costs of privacy: surveillance and reputation in America Hawthorne: Walter de Gruyter

Nordenfelt, L. (1995). On the nature of health. An action theoretic approach Dordrecht: Kluwer Academic Publishing

Nordenfelt, L. (2007). The concepts of health and illness revisited. Medicine, Health Care and Philosophy, 10, 5-10.

Office for National Statistics (2000). Key health statistics from general practice 1998. Analyses of morbidity and treatment data, including time trends, England and Wales. London: Office for National Statistics.

Office for National Statistics (2005). All fields postcode directory. London.

Office for National Statistics (2006a). Cancer Statistics registrations: Registrations of cancer diagnosed in 2004, England. London: Office for National Statistics.

Office for National Statistics (2006b). The 2001 Census: Office for National Statistics.

Office for National Statistics (2008a). Key population and vital statistics. London.

Office for National Statistics (2008b). Health Survey for England 2006. London.

Ohm, P. (2008). The myth of the superuser: fear, risk and harm online. University of California Davis School of Law, 41(4), 1327-1402.

Openshaw, S. (1984). The modifiable areal unit problem, Concepts and techniques in modern geography. London: Geo Books.

Openshaw, S., Charlton, M., Wymer, C., \& Craft, A. (1987). A mark 1 Geographical Analysis Machine for the automated analysis of point data sets. International Journal of Geographical Information Systems, 1, 335-358.

Openshaw, S. (1991). Developing approriate spatial analysis methods for GIS. In D.J. Maguire,M.F. Goodchild, \& D.W. Rhind (Eds.), Geographical Information Systems (pp. 389-402). Harlow: Longman Scientific \& Technical.

Openshaw, S. (1995). Geodemographic segmentation systems for screening health data. Journal of Epidemiogy and Community Health, 49(Supplement 2), S34-S38.

Oppenheimer, G.M. (2001). Paradigm lost: race, ethnicity, and the search for a new population taxonomy. American Journal of Public Health, 91(7), 1049-1055.

Organisation for Economic Cooperation and Development (2005). Health data. Paris: OECD. 
Osborne, R. (2006). Civilization London: Pimlico

Paradies, Y.C., Montoya, M.J., \& Fullerton, S.M. (2007). Racialized Genetics and the Study of Complex Diseases - the thrifty genotype revisited Perspectives in Biology and Medicine, 50(2), 203-227.

Pearce, J., Witten, K., Hiscock, R., \& Blakely, T. (2007). Are socially disadvantaged neighbourhoods deprived of health-related community resources? Int J Epidemiol, 36(2), 348 - 355.

Pearson, M., Sweeting, H., West, P., Young, R., Gordon, J., \& Turner, K. (2006). Adolescent substance use in different social and peer contexts: A social network analysis. Drugs: education, prevention and policy, 13(6), 519 - 536.

Pedhazur, E., \& Pedhazur Schmelkin, L. (1991). Measurement, Design, and Analysis: An Integrated Approach Mahwah: Lawrence Erlbaum Associates

Petersen, J., Mateos, P., Longley, P., \& Atkinson, P. (2008). Inclusion and exclusion problems in geodemographic targeting, Geographical Information Science Research UK. Manchester Metropolitan University.

Philips, D.J., \& Curry, M.R. (2003). Privacy and the phenetic urge: geodemographics and the changing spatiality of local practice. In D. Lyon (Ed.), Surveillance as Social Sorting: Privacy, Risk, and Digital Discrimination (pp. 137-152). London: Routledge.

Pickett, K.E., \& Pearl, M. (2001). Multilevel analyses of neighbourhood socio-economic context and health outcomes: a critical review. Journal of Epidemiology and Community Health, 55, 111 - 122.

Pickles, J. (1994). Ground Truth: the social implications of geographical information systems New York: Guilford Press

Platt, L., Simpson, L., \& Akinwale, B. (2005). Stability and change in ethnic groups in England and Wales. Population Trends, 121, 35.

Pollock, A.M., Price, D., Viebrock, E., Miller, E., \& Watt, G. (2007). The market in primary care. British Medical Journal, 335, 475-477.

Preamble to the constitution of the World Health Organisation (1948). WHO definition of Health: International Health Conference, New York, 19-22 June, 1946; signed on 22 July 1946 by the representatives of 61 States (Official Records of the World Health Organization, no. 2, p. 100) and entered into force on 7 April 1948.

Rabe-Hesketh, S., \& Everitt, B. (2004). A handbook of statistical analyses using Stata Boca Raton: Chapman \& Hall, CRC

Rankin, J., \& Bhopal, R. (1999). Current census categories are not a good match for identity. British Medical Journal, 318(7199), 1696-.

Rawlins, M. (2008). Assessing the health-related quality of life (lecture), Pharmacoepidemiology and pharmacovigilance. London School of Hygiene and Tropical Medicine (12 November 2008).

Rawls, J., \& Kelly, E. (2001). Justice as Fairness: A Restatement Cambridge (MA): Harvard University Press

Razum, O., Zeeb, H., Akgun, H.S., \& Yilmaz, S. (1998). Low overall mortality of Turkish residents in Germany persists and extends into a second generation: merely a healthy migrant effect?*. Tropical Medicine \& International Health, 3(4), 297-303. 
Reid, D.J., Killoran, A.J., McNeill, A.D., \& Chambers, J.S. (1992). Choosing the most effective health promotion options for reducing a nation's smoking prevalence. Tobacco Control, 1, 185-197.

Remennick, L. (2006). The Challenge of Early Breast Cancer Detection among Immigrant and Minority Women in Multicultural Societies. The Breast Journal, 12(1), S103-S110.

Rhodes, R. (2004). Justice in allocations for terrorism, biological warfare, and public health. In M. Boylan (Ed.), Public health policy and ethics. Dordrecht: Kluwer Academic Publishers.

Ridley, M. (2003). Nature via nurture London: Fourth Estate Ltd.

Riva, M., Gauvin, L., \& Barnett, T.A. (2007). Toward the next generation of research into small area effects on health: a synthesis of multilevel investigations published since July 1998. Journal of Epidemiogy and Community Health, 61(10), 853-861.

Sadurski, W. (2007). Welfare, resources, and Luck Egalitarianism. European University Institute Working Papers, 2007(05).

Samuelson, P.A. (1954). The pure theory of public expenditure. Review of Economics and Statistics, 36, 387-389.

Sankar, P., \& Cho, M.K. (2002). Toward a New Vocabulary of Human Genetic Variation. Science, 298(5597), 1337-1338.

Saxena, S., George, J., Barber, J., Fitzpatrick, J., \& Majeed, A. (2006). Association of population and practice factors with potentially avoidable admission rates for chronic diseases in London: cross sectional analysis. Journal of the Royal Society of Medicine, 99, 81-89.

Schuurman, N. (1999). Trouble in the heartland: GIS and its critics in the 1990s. Progress in Human Geography, 24(4), 569-590.

Schuurman, N. (2006). Formalization matters: critical GIS and ontology research. Annals of the American Geographers, 96(4), 726-739.

Schuurman, N., Fiedler, R., Grzybowski, S., \& Grund, D. (2006). Defining rational hospital catchments for non-urban areas based on travel-time. International Journal of Health Geographics, 5(43), 1-11.

Schuurman, N., \& Leszczynski, A. (2008). Ontologies and the reorganization of biological databases in the age of bioinformatics. Bioinformatics and Biology Insights, 2, 187-200.

Seedhouse, D. (2004). Health Promotion: Philosophy, Prejudice and Practice Chichester: John Wiley and Sons

Sempere-Selva, T., Peiro, S., Sendra-Pina, P., Martinez-Espin, C., \& Lopez-Aguilera, I. (2001). Inappropriate use of an accident and emergency department: magnitude, associated factors, and reasons - an approach with explicit criteria. Annals of Emergency Medicine, 37, 568-579.

Sen, A. (1993). Capability and wellbeing. In M.C. Nussbaum, \& A. Sen (Eds.), The quality of life (pp. 30-54). Oxford: Clarendon Press.

Seng, K.C., \& Seng, C.K. (forthcoming). The success of the genome-wide association approach: a brief story of a long struggle. European Journal of Human Genetics.

Shaw, M., \& Dorling, D. (2004). Who cares in England and Wales? The positive care law: cross-sectional study. British Journal of General Practice, 54, 899-903. 
Shetty, P. (2001). Variation in health and disease: race, ethnicity or 'nutrition transition', Health and ethnicity (pp. 147-163). London: Taylor and Francis.

Siegrist, J., \& Marmot, M. (2006). Social inequalities in health - new evidence and policy implications. Oxford: Oxford University Press.

Silverman, B.W. (1986). Density estimation for statistics and data analysis London: Chapman and Hall

Singleton, A., \& Longley, P.A. (2008). Creating Open Source Geodemographic Classifications for Higher Education Applications. CASA Working Paper, 133.

Skipper, M. (2007). Genomics: HapMap Phase II unveiled. Nature Reviews Genetics, 8(11), 826-827.

Sleight, P. (2004). Targeting customers. How to use geodemographics and lifestyle data in your business Henley-on-Thames: WARC

Smedley, A., \& Smedley, B.D. (2005). Race as Biology Is Fiction, Racism as a Social Problem Is Real - Anthropological and Historical Perspectives on the Social Construction of Race. American Psychologist, 54(10), 805-816.

Smedley, B.D., Stith, A.Y., \& Nelson, A.R. (2003). Unequal treatment: confronting racial and ethnic disparities in health care Washington DC: Institute of Medicine

Social Exclusion Unit (1999). Teenage pregnancy report: Social Exclusion Unit, Cabinet Office.

Social Exclusion Unit (2006). Social Exclusion Unit.

Speller, V., \& Hale, D. (1985). Making the most of your postcode. Health Services Journal, 28, 53.

Spencer, G. (2008). International Consortium Announces the 1000 Genomes Project. Major Sequencing Effort Will Produce Most Detailed Map Of Human Genetic Variation to Support Disease Studies: International Consortium for the 1000 Genomes Project.

Sriskandarajah, D., \& Drew, C. (2006). Brits abroad: mapping the scale and nature of British emigration: Institute for Public Policy Research

Swann, C., Bowe, K., McCormick, G., \& Kosmin, M. (2003). Teenage pregnancy and parenthood: a review of reviews. London: Health Development Agency.

Szczepura, A. (2005). Access to health care for ethnic minority populations. Postgraduate Medical Journal, 81(953), 141-147.

Talbot-Smith, A., \& Pollock, A.M. (2006). The New NHS - a guide London: Routledge

Tate, S.K., \& Goldstein, D.B. (2004). Will tomorrow's medicines work for everyone? Nature Genetics, 36, S34-S42.

Thakur, R., \& Weiss, T.G. (2005). The UN and Global Governance: An Idea and its Prospects Bloomington: Indiana University Press

Thorsen, D., \& Lie, A. (2007). What is neoliberalism?, Nyliberalisme - ideer og politisk virkelighet (pp. 33-48). Oslo: Oslo Universitetsforlaget.

Townsend, P. (1979). Poverty in the UK Harmondsworth: Penguin

Tripp, J., \& Viner, R. (2005). ABC of adolescence: sexual health, contraception, and teenage pregnancy. British Medical Journal, 330, 590-593.

Twigg, L. (1989). Health based geographical information systems: their potential examined in the light of existing data sources. Social Science and Medicine, 30, 143-155. 
UNAIDS (2006). AIDS epidemic update 2007. Geneva: Joint United Nations Programme on HIV/AIDS (UNAIDS) and World Health Organization (WHO) 2007.

Underwood, M.J., \& Bailey, J.S. (1993). Coronary bypass surgery should not be offered to smokers. British Medical Journal, 306(6884), 1047-1049.

USA Today (2008). Targeting Opportunities. New York: USATODAY.COM.

Van Oudenhoven, J.P., Ward, C., \& Masgoret, A.-M. (2006). Patterns of relations between immigrants and host societies. International Journal of Intercultural Relations, 30(6), 637-651.

Verweij, M. (2007). Tobacco discourament: a non-paternalistic argument, Ethics, Prevention, and Public Health. Oxford: Clarendon Press.

Vickers, D., Rees, P., \& Birkin, M. (2005). Creating the national classification of census output areas: data, methods and results, Working paper. Leeds: School of Geography, University of Leeds.

Wagner, H. (1998). Chronic disease management: What will it take to improve care fro chronic illness? Effective Clinical Practice, 1(1), 2-4.

Wallace, P., Bowling, A., \& Roberts, J.A. (2006). Better services and more choice in the NHS. British Medical Journal, 333, 56-57.

Waller, W., \& Gotway, C.A. (2004). Applied Spatial Statistics for Public Health Data New York: Wiley

Walters, G.D. (2006). Lifestyle theory - present and future New York: Nova Publishers

Weathers (2004). Children of migratory agricultural workers: the ecological context of acute care for a mobile population of immigrant children. Clinical Pediatric Emergency Medicine, 5, 120-129.

Webber, R., \& Longley, P. (2003). Geodemographic analyisis of similarity and proximity: their roles in the understanding of the geography of need. In M. Batty, \& P. Longley (Eds.), Advanced spatial analysis: the CASA book of GIS (pp. 233266). Redlands: ESRI Press.

Weinstein, M.C., \& Manning, W.G. (1997). Theoretical issues in cost-effectiveness analysis. Journal of Health Economics, 16(1), 121-128.

Wellman, C. (2002). The proliferation of rights. Moral progress or empty rhetoric? In L. Gearon (Ed.), Human rights and religion. Brighton: Sussex Academic Press.

West, P., Sweeting, H., \& Leyland, A. (2004). School effects on pupils' health behaviours: evidence in support of the health promoting school. Research Papers in Education, 19(3), 261 - 291.

Wilkinson, R.G. (2005). The Impact of Inequality: How to Make Sick Societies Healthier Abingdon: Routledge

Winkler, W.E. (2006). Overview of record linkage and current research directions. Washington: US Census Bureau, Statistical Research Division.

Winslow, C.E.A. (1920). The untilled fields of public health. Science, $51,23$.

Witten, I., \& Frank, E. (2005). Data Mining: Practical Machine Learning Tools and Techniques San Francisco: Morgan Kaufmann

World Health Organisation (1998). HEALTH21: an introduction to the health for all policy framework, European Health for All 5. Copenhagen: WHO Regional Office for Europe. 


\section{Appendices: Refereed journal articles}

A1: Petersen, J., Atkinson, P., Petrie, S., Gibin, M., Ashby, D., Longley, P. (2009) Teenage pregnancy - new tools to support local health campaigns. Health and Place 15(1), 300-307.

A2: Preventable A\&E usage - The potentials of using a name-based ethnicity classification, Onomap, in identifying user groups at King College's Hospital, London UK. Petersen, J., Gibin, M., Mateos, P., Atkinson, P., Longley, P. submitted to Social Science and Medicine 